\title{
Understanding the Subtleties of Frustrated Lewis Pair Activation of Carbonyl Compounds by N-Heterocyclic Carbene/Alkyl Gallium
}

\section{Pairings}

\author{
Marina Uzelac, ${ }^{[a]}$ David R. Armstrong, ${ }^{[a]}$ Alan R. Kennedy ${ }^{[a]}$ and Eva Hevia*[a]
}

\begin{abstract}
This study reports the use of the trisalkylgallium $\mathrm{GaR}_{3}(R=$ $\mathrm{CH}_{2} \mathrm{SiMe}_{3}$ ), containing sterically demanding monosilyl groups, as an effective Lewis acid component for Frustrated Lewis Pair activation of carbonyl compounds, when combined with the bulky $\mathrm{N}$-heterocyclic carbene 1,3-bis(tert-butyl)imidazol-2-ylidene (It'Bu) or 1,3-bis(tertbutyl)imidazolin-2-ylidene ( $\mathrm{SI} I^{\prime} \mathrm{Bu}$ ). The reduction of aldehydes can be achieved by insertion into the $\mathrm{C}=\mathrm{O}$ functionality via the $\mathrm{C} 2$ (so-called normal) position of the carbene affording zwitterionic products $\left[{ }^{t} \mathrm{BuCH}_{2} \mathrm{OGaR}_{3}\right](1)$ or $\left[\mathrm{I}^{\mathrm{t}} \mathrm{BuCH}\left(\mathrm{p}-\mathrm{Br}-\mathrm{C}_{6} \mathrm{H}_{4}\right) \mathrm{OGaR}_{3}\right](2)$ or alternatively via its abnormal (C4) site yielding [all ${ }^{\mathrm{BuCH}}\left(\mathrm{p}-\mathrm{Br}_{-} \mathrm{C}_{6} \mathrm{H}_{4}\right) \mathrm{OGaR}_{3}$ ] (3). Proving the cooperative behaviour of both components, It $\mathrm{Bu}$ and $\mathrm{GaR}_{3}$, when separated neither of them are able to activate any of these substrates. NMR spectroscopic studies of the new compounds point to complex equilibria involving the formation of kinetic and thermodynamic species as implicated through the aid of DFT calculations. Extension to ketones proved to be successful for electrophilic a,a,a-trifluoroacetophenone yielding [alt $\left.\mathrm{BuC}(\mathrm{Ph})\left(\mathrm{CF}_{3}\right) \mathrm{OGaR}_{3}\right](7)$; however in the case of ketones and nitriles bearing acidic hydrogen atoms, $\mathrm{C}-\mathrm{H}$ bond activation takes place preferentially, affording novel imidazolium gallate salts such as $\left[\left\{I^{t} \mathrm{BuH}\right\}^{+}\left\{\left(\mathrm{p}-\mathrm{I}-\mathrm{C}_{6} \mathrm{H}_{4}\right) \mathrm{C}\left(\mathrm{CH}_{2}\right) \mathrm{OGaR}_{3}\right\}^{-}\right](8)$ or $\left[\left\{I^{t} \mathrm{BuH}\right\}^{+}\left\{\mathrm{Ph}_{2} \mathrm{C}=\mathrm{C}=\mathrm{NGaR}_{3}\right\}^{-}\right]$
\end{abstract} (12).

\section{Introduction}

The chemistry of sterically mismatched Lewis acid-base pairs capable of unusual reactivities, today commonly referred as "frustrated Lewis pairs (FLPs)", has been brought to the forefront of main group chemistry by the seminal work of Stephan and coworkers. ${ }^{[1,2]}$ Since then, the field of FLP chemistry has snowballed due in part to the ever-growing scope of small molecules that can be activated (e.g., $\mathrm{H}_{2}$ and greenhouse gasses), and also because of its progression from stoichiometric to catalytic processes. ${ }^{[2, f]}$ Although the most powerful FLPs to date rely on the use of sterically hindered, electron-rich organophosphines as the Lewis base (LB) component, ${ }^{[1-3]} \mathrm{N}$-heterocyclic carbenes (NHCs) are increasingly gaining attention due to their related coordination chemistry, tuneability and excellent potential for subtle variations of their steric/electronic properties. ${ }^{[4-6]}$ The Lewis acidic component of these pairs is still heavily dominated by boron

[a] M. Uzelac, Dr. D. R. Armstrong, Dr. A. R. Kennedy, Prof. E. Hevia WestCHEM, Department of Pure and Applied Chemistry University of Strathclyde 295 Cathedral Street, Glasgow, UK, G1 1XL. E-mail: eva.hevia@strath.ac.uk

Supporting information for this article is given via http://dx.doi.org/10.1002/chem2015xxxx. complexes, with aluminium species showing slow but steady growth in popularity. ${ }^{[7-9]}$ By comparison, the use of heavier gallium Lewis acids (LA) has barely started. In this context, studies on the reaction of 4-ethynyl-2,6-lutidine with a range of group 13 compounds have revealed that while using $\mathrm{B}\left(\mathrm{C}_{6} \mathrm{~F}_{5}\right)_{3}$ leads to FLP activation processes, alkyl complexes of the heavier $\mathrm{Al}, \mathrm{Ga}$ and In display a common but distinct reactivity, promoting metalation of the ethynyl group with concomitant elimination of the relevant alkane. ${ }^{[10]}$ Breaking new ground in the field, Aldridge has reported an ambiphilic gallium system, containing an activated $\beta$ diketiminate ligand, capable of cooperative activation of protic, hydridic and apolar $\mathrm{H}-\mathrm{X}$ bonds. ${ }^{[1]}$ Furthermore, the enhanced Lewis acidity of $\mathrm{Ga}$ alkyl fragments has proved to be key for promoting direct $\mathrm{Ge}-\mathrm{H}$ exchange of phenylacetylene using mixed $\mathrm{Ga} / \mathrm{Ge}$ complexes. ${ }^{[12 a]}$ Interesting recent work by Schultz has also uncovered the double agent role of $\mathrm{Ga}$ systems as they can reverse their role in FLP chemistry, acting instead as a LB, being part of a gallanedyl framework, which facilitates insertion of benzaldehyde when combined with $\left[\mathrm{M}\left(\mathrm{C}_{6} \mathrm{~F}_{5}\right)_{3}\right](\mathrm{M}=\mathrm{B}$ or $\mathrm{Al})$ LAs. ${ }^{[13]}$

Advancing rational design of elusive main group abnormal $\mathrm{NHC}$ (aNHC) complexes, our first look at NHC-Ga alkyl complexes shed light on normal-to-abnormal $\mathrm{NHC}$ rearrangement mechanisms, ${ }^{[14]}$ which bears resemblance to mechanisms proposed in NHC/borane FLP systems. ${ }^{[4]}$ In particular, it was found that steric incompatibility prevented trimethylsilylmethylgallium(III) $\left(\mathrm{GaR}_{3}\right)$ and 1,3-bis(tertbutyl)imidazol-2-ylidene (ItBu) from forming a stable normal adduct. ${ }^{[14]}$ Encouraged by these initial findings, here we present a systematic study probing the reactivity of these $\mathrm{NHC} / \mathrm{Ga}$ combinations towards carbonyl compounds, an area where other, mostly, but not limited to, $\mathrm{PR}_{3} / \mathrm{B}$ based FLP systems have shown considerable promise in synthesis and catalysis. ${ }^{[13,15-17]}$ Combining X-ray crystallographic with spectroscopic and theoretical investigations, new insights are provided into the intriguing chemoselectivities and mechanisms of these processes, which intensifies the potential of gallium tris(alkyl) complexes for FLP activation.

\section{Results and Discussion}

\section{Reactions with aldehydes}

We started by reacting $\mathrm{GaR}_{3} / \mathrm{I}^{\mathrm{B}} \mathrm{Bu}$ with the simplest and most reactive aldehyde, namely paraformaldehyde, in hexane suspension at $0{ }^{\circ} \mathrm{C}$. Mixing all three components immediately produced a white precipitate which could be solubilised by addition of toluene to obtain X-ray amenable crystals of zwitterion $\left[{ }^{t} \mathrm{BuCH}_{2} \mathrm{OGaR}_{3}\right] 1$ in a $66 \%$ yield (Scheme $1 \mathrm{a}$ ). 


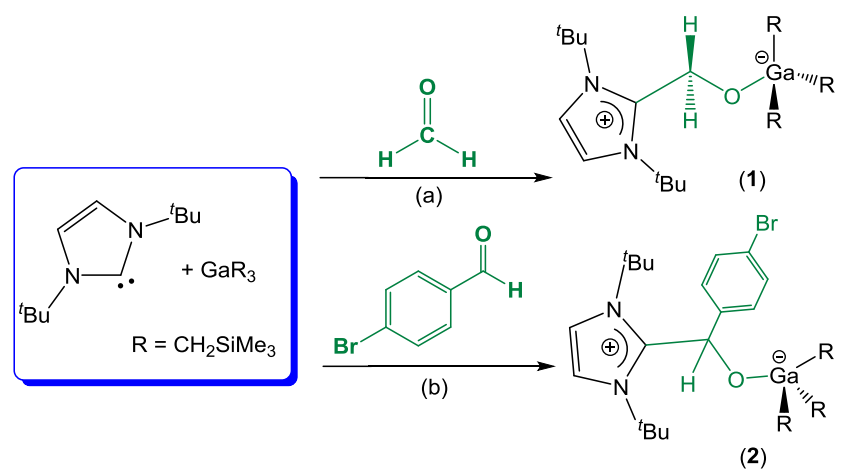

Scheme 1. FLP-induced synthesis of zwitterionic compounds 1 and 2

A single crystal $X$-ray diffraction analysis (Fig 1 ) revealed addition of the $I^{t} \mathrm{Bu} / \mathrm{GaR}_{3}$ pair across the $\mathrm{C}=\mathrm{O}$ functionality to construct $\mathrm{a}$ new, single carbon-carbon bond [i.e., C1-C24, 1.505(3) $\AA$ ] and an oxygen-gallium bond. The $\mathrm{C}_{3} \mathrm{O}$-tetra-coordinated gallium atom adopts a distorted tetrahedral geometry [bond angles ranging from $102.90(9)^{\circ}$ to $116.28(11)^{\circ}$ : mean value, $109.16^{\circ}$ ]. Ga- $\mathrm{C}_{\text {alkyl }}$ and $\mathrm{Ga}-\mathrm{O}$ bond distances [mean Ga-C, $2.012 \AA ; \mathrm{Ga} 1-\mathrm{O} 1$, $1.9317(15) \AA]$ are in good agreement with literature values of other tetracoordinated gallium species. ${ }^{[14,18]}$
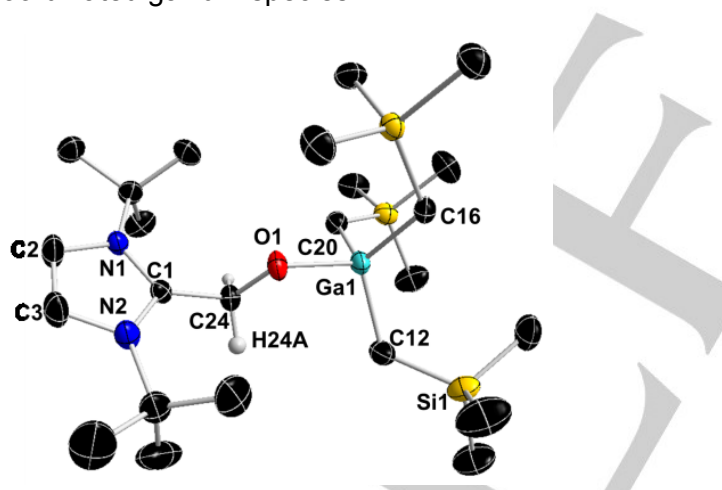

Figure 1. Molecular structure of 1 with $50 \%$ probability displacement ellipsoids. All $\mathrm{H}$ atoms except those in the starting carbonyl group have been omitted for clarity. Selected bond distances $(\AA)$ and bond angles $\left({ }^{\circ}\right)$ : $\mathrm{Ga}(1)-\mathrm{O}(1)$ 1.9317(15), $\mathrm{Ga}(1)-\mathrm{C}(12)$ 2.012(3), Ga(1)-C(16) 2.012(2), Ga(1)-C(20) 2.011(3), O(1)-C(24) 1.384(3), C(1)-C(24) 1.505(3), O(1)-Ga(1)-C(20) 103.41(9), O(1)-Ga(1)-C(12) 105.21(9), C(20)-Ga(1)-C(12) 110.86(13), O(1)-Ga(1)-C(16) 102.90(9), C(20)$\mathrm{Ga}(1)-\mathrm{C}(16) \quad 116.27(11), \quad \mathrm{C}(12)-\mathrm{Ga}(1)-\mathrm{C}(16) \quad 116.28(11), \quad \mathrm{N}(1)-\mathrm{C}(1)-\mathrm{N}(2)$ 107.50(19).

The most informative resonance in the ${ }^{1} \mathrm{H}$ NMR spectrum in $\mathrm{d}_{8-}$ THF is a singlet at $5.05 \mathrm{ppm}$ for formerly aldehydic protons (i.e. $\mathrm{H} 24 \mathrm{~A}$ in Fig. 1), which displays a significant upfield shift (vs. 9.58 $\mathrm{ppm}$ in free formaldehyde). Similarly, in the ${ }^{13} \mathrm{C}$ NMR spectrum, the resonances of former carbenic and $\mathrm{C}=\mathrm{O}$ fragments are observed at $153.2 \mathrm{ppm}$ and $58.6 \mathrm{ppm}$, respectively (vs. $213.2 \mathrm{ppm}$ and $195.2 \mathrm{ppm}$ in the free reagents).
Applying the same protocol to the aromatic aldehyde 4bromobenzaldehyde, afforded $\left[{ }^{t} \mathrm{BuCH}\left(p-\mathrm{Br}-\mathrm{C}_{6} \mathrm{H}_{4}\right) \mathrm{OGaR}_{3}\right](2)$ in $42 \%$ crystalline yield (Scheme $1 \mathrm{~b}$ ). As evidenced by X-ray crystallographic studies, 2 (Fig. 2) has the same molecular structure as 1 and displays similar gross structural features.

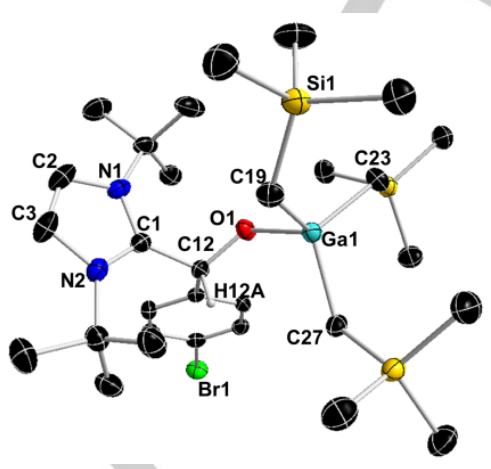

Figure 2. Molecular structure of $\mathbf{2}$ with $50 \%$ probability displacement ellipsoids All $\mathrm{H}$ atoms except that on the former carbonyl group have been omitted for clarity. Selected bond distances $(\AA)$ and bond angles $\left({ }^{\circ}\right): \mathrm{Ga}(1)-\mathrm{O}(1) 1.9522(16)$, $\mathrm{Ga}(1)-\mathrm{C}(19)$ 2.021(2), Ga(1)-C(23) 2.005(2), Ga(1)-C(27) 2.027(2), O(1)-C(12) 1.372(3), C(1)-C(12) 1.525(3), O(1)-Ga(1)-C(23) 105.42(9), O(1)-Ga(1)-C(19) 103.48(9), C(23)-Ga(1)-C(19) 114.57(10), O(1)-Ga(1)-C(27) 102.42(8), C(23)$\mathrm{Ga}(1)-\mathrm{C}(27) \quad 111.41(11), \quad \mathrm{C}(19)-\mathrm{Ga}(1)-\mathrm{C}(27) \quad 117.58(10), \quad \mathrm{N}(1)-\mathrm{C}(1)-\mathrm{N}(2)$ 107.8(2).

Although in the solid state $\mathbf{2}$ is molecularly isostructural with $\mathbf{1}$, NMR spectroscopic analyses revealed a much more complex scenario in solution. As 2 exhibited poor solubility in arene solvents such as $\mathrm{C}_{6} \mathrm{D}_{6}$, its solution studies were conducted in $d_{8^{-}}$ THF where two different species containing reduced carbonyl group were observed together with the free starting materials. ${ }^{[19]}$ One species can be confidently assigned to complex $\mathbf{2}$, displaying informative resonances in the ${ }^{13} \mathrm{C}$ NMR spectrum at 72.3 and $154.2 \mathrm{ppm}$ for the $\mathrm{OCH}(\mathrm{Ar})$ and $\mathrm{NCN}$ fragments respectively. Interestingly, over the course of 24 hours, ${ }^{[20]} \mathbf{2}$ evolved completely into the new complex (Fig S32 in $\mathrm{SI}$ ), which could be isolated and structurally defined as [al' $\left.\mathrm{BuCH}\left(p-\mathrm{Br}-\mathrm{C}_{6} \mathrm{H}_{4}\right) \mathrm{OGaR}_{3}\right]$ (3) (Fig. 3).

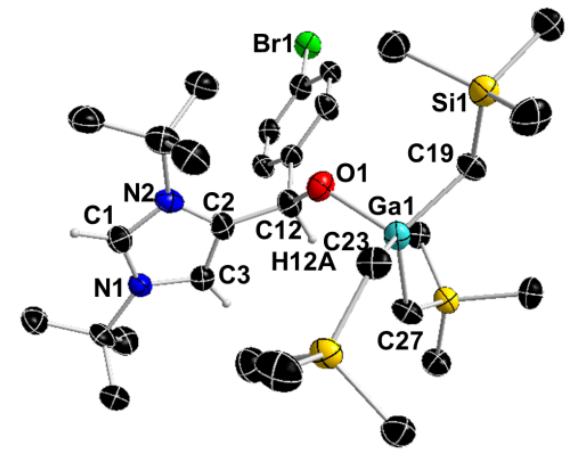

Figure 3. Molecular structure of $\mathbf{3}$ with $50 \%$ probability displacement ellipsoids. All $\mathrm{H}$ atoms except those of the imidazole ring and the former carbonyl group have been omitted for clarity. Selected bond distances $(\AA)$ and bond angles $\left({ }^{\circ}\right)$ : $\mathrm{Ga}(1)-\mathrm{O}(1)$ 1.943(5), Ga(1)-C(19) 2.019(8), Ga(1)-C(23) 2.020(7), Ga(1)-C(27) 2.012(8), $\mathrm{O}(1)-\mathrm{C}(12) \quad 1.335(9), \quad \mathrm{C}(2)-\mathrm{C}(12) \quad 1.541(10), \quad \mathrm{O}(1)-\mathrm{Ga}(1)-\mathrm{C}(27)$ 109.6(3), O(1)-Ga(1)-C(19) 102.0(3), C(27)-Ga(1)-C(19) 114.4(3), O(1)-Ga(1)- 
C(23) 102.8(3), C(27)-Ga(1)-C(23) 113.3(3), C(19)-Ga(1)-C(23) 113.3(3), N(1)$\mathrm{C}(1)-\mathrm{N}(2) 109.9(7)$

Being constitutionally isomeric with $\mathbf{2}$, complex $\mathbf{3}$ also contains an $\mathrm{OCHAr}$ fragment coordinated to $\mathrm{Ga}$, resulting from the addition of the carbene across the $\mathrm{C}=\mathrm{O}$ bond of the aldehyde. However in this case, this has occurred via one of the $\mathrm{C}$ atoms located at the backbone of the imidazole ring (so called abnormal[ ${ }^{[21 a, 22]}$ or ${ }^{\mathrm{C}} 4-$ position) [C2 in Fig. 3], whereas the former carbenic $\mathrm{C}$ in It Bu [C1 in Fig. 3] is now protonated. Consistent with the lack of symmetry in the imidazole ring, ${ }^{1} \mathrm{H}$ NMR spectrum of 3 in $d_{8}$-THF showed two singlets for the non-equivalent protons of the imidazole ring at 8.45 and $7.84 \mathrm{ppm}$. Furthermore, the ${ }^{13} \mathrm{C}$ NMR spectrum displays a resonance at $72.7 \mathrm{ppm}$ which can be assigned to the reduced $\mathrm{C}=\mathrm{O}$ functionality (i.e., $\mathrm{C} 12$ in Fig. 3), whereas the formally $\mathrm{C} 2$ and $\mathrm{C} 4$ carbons of $\mathrm{I}^{\mathrm{t}} \mathrm{Bu}$ (i.e., $\mathrm{C} 1$ and $\mathrm{C} 2$ in Fig. 3) resonate at 131.8 and $149.0 \mathrm{ppm}$ respectively.

Complexes 2 and 3 can be described as kinetic and thermodynamic products of the activation of 4bromobenzaldehyde by $\mathrm{GaR}_{3} / \mathrm{I}^{t} \mathrm{Bu}$ combinations. Supporting this interpretation, monitoring the reaction over a range of temperatures revealed, that at $230 \mathrm{~K}$, by mixing $\mathrm{GaR}_{3}$ and the aldehyde, an adduct must be formed (as all resonances are slightly upfield when compared to those of the free starting materials). Under these conditions, addition of $I^{t} \mathrm{Bu}$ led to the almost instantaneous formation of 2. Interestingly, by increasing the temperature to $323 \mathrm{~K}$ we observed full conversion of $\mathbf{2}$ into $\mathbf{3}$ over just 15 minutes (see Fig S15-17 in SI). During these solution studies, the persistent presence of small amounts of the three components of these complexes, I ${ }^{t} \mathrm{Bu}, \mathrm{GaR}_{3}$ and $\mathrm{ArCHO}$, suggested that all these species co-exist in equilibrium. Further support was found by dissolving isolated crystals of 3 in $d_{8}$-THF the resulting ${ }^{1} \mathrm{H}$ NMR spectrum of which showed, once again, the presence of resonances for the three starting materials. These findings are consistent with the reversible cleavage in solution of the newly formed $\mathrm{C}-\mathrm{C}$ and $\mathrm{Ga}-\mathrm{O}$ bond in both adducts 2 and $\mathbf{3}$. It should be noted that a similar equilibrium has been reported previously by Erker for the reactions of geminal P/B FLP systems with benzaldehyde and trans-cinammic aldehyde. ${ }^{[15]}$

By irrefutably establishing interconversion of $\mathbf{2}$ into $\mathbf{3}$, it was possible to prepare 3 rationally from starting materials in hexane at $0{ }^{\circ} \mathrm{C}$ simply by extending the reaction time from 15 minutes to two hours (Scheme 2).

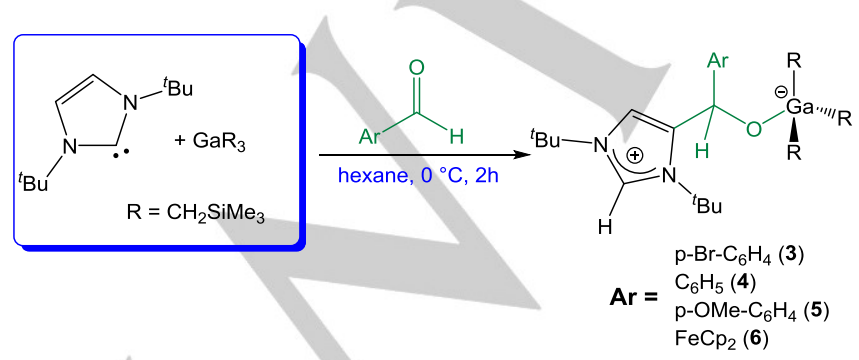

Furthermore, this approach to access C4-substituted abnormal adducts can be successfully extended to other aldehydes, including benzaldehyde, 4-anisaldehyde and ferrocenecarboxaldehyde affording alkoxo derivatives [alt $\mathrm{BuCH}\left(\mathrm{C}_{6} \mathrm{H}_{5}\right) \mathrm{OGaR}_{3}$ ] (4), [al't $\mathrm{BuCH}\left(p-\mathrm{OMe}-\mathrm{C}_{6} \mathrm{H}_{4}\right) \mathrm{OGaR}_{3}$ ] (5) and $\left[a{ }^{t} \mathrm{BuCH}\left(\mathrm{FeCp}_{2}\right) \mathrm{OGaR}_{3}\right](6)$ in a 62,56 and $48 \%$ isolated crystalline yield respectively (Scheme 2 ).

Multinuclear NMR spectroscopic studies confirmed that in all cases the insertion of the substrate has taken place via the $I^{t} \mathrm{Bu}$ C4-position (see SI for details). Mimicking the situation with 3, activation products 4-6 are in equilibrium with their free components when dissolved in $d_{8}$-THF. Interestingly, the position of this dissociative equilibrium seems to correlate with the electronic nature of the Ar group substituents of the aldehyde. Thus, by replacing the electron-withdrawing bromide with $\mathrm{H}$ (in 4) or electron-donating $\mathrm{OMe}$ (in 5) leads to higher ratios of free $\mathrm{I}^{t} \mathrm{Bu}$, $\mathrm{GaR}_{3}$ and the relevant aldehyde. On the other hand, with 6, which contains a ferrocenyl substituent, no measurable sign of dissociation is observed. An interesting solvent effect was also evident in these processes. Contrasting with the studies using $d_{8}$ THF, when $\mathrm{C}_{6} \mathrm{D}_{6}$ was employed compound 4 (which is the only one partially soluble in this arene solvent), showed no evidence of being in equilibrium with its free components, suggesting that donor solvents such as THF favour cleavage of the complex.

\section{Mechanistic implications and DFT calculations}

Previous studies using NHC-based FLP systems containing $\mathrm{B}^{[4 \mathrm{e}}$, ${ }^{23]}$ and $\mathrm{Al}\left[{ }^{[7 a}\right.$, 9a] LAs have shown their ability to reduce $\mathrm{CO}_{2}$ and other carbonyl compounds using either the $\mathrm{C} 2$ or $\mathrm{C} 4$ position of their NHC component. However, as far as we are aware, this is the first time that both possible constitutional isomers (i.e., 2 and 3) have been isolated and structurally defined. Furthermore, our investigations also indicate that the formation of the C4-inserted products is thermodynamically controlled. It should also be stressed that $I^{t} \mathrm{Bu}$ is not capable of activating any of the studied substrates on its own. Also despite the fact that our solution studies evidence the presence of variable amounts of free $I^{t} \mathrm{Bu}$ and $\mathrm{GaR}_{3}$, the abnormal complex [(alt $\left.\left.\mathrm{Bu}\right) \mathrm{GaR}_{3}\right]$ (which is observed as the only product when both reagents are mixed in the absence of the aldehyde) ${ }^{[14]}$ has not been detected.

Intrigued by these findings we have carried DFT calculations ${ }^{[24]}$ employing the B3LYP method ${ }^{[25]}$ and the $6-311 G(d, p)$ basis set ${ }^{[26]}$ to optimise structures and cast light on the thermodynamics involved in these processes. Geometrical parameters of optimised structures [ $\left.\mathrm{I}^{t} \mathrm{BuCH}\left(\mathrm{p}-\mathrm{Br}-\mathrm{C}_{6} \mathrm{H}_{4}\right) \mathrm{OGaR}_{3}\right] \quad\left(\mathrm{II}_{\mathrm{Ar}}\right)$ and [al ${ }^{t} \mathrm{BuCH}\left(\mathrm{p}-\mathrm{Br}-\mathrm{C}_{6} \mathrm{H}_{4}\right) \mathrm{OGaR}_{3}$ ] (III $\left.\mathrm{Ar}_{\mathrm{Ar}}\right)$ are in excellent agreement with those obtained experimentally by $\mathrm{X}$-ray analysis of $\mathbf{2}$ and $\mathbf{3}$, respectively (Table S3), with only a slight underestimation of the strength of the Ga-O interaction $\left(\Delta\left[\mathrm{d}(\mathrm{Ga}-\mathrm{O})_{\text {calc }}-\mathrm{d}(\mathrm{Ga}-\mathrm{O})_{\text {exp }}\right]=\right.$ 0.063 and $0.057 \AA$ for $\mathrm{II}_{\mathrm{Ar}}$ and $\mathrm{III}_{\mathrm{Ar}}$ respectively). Interestingly, consistent with experimental observations, model $\mathrm{III}_{\mathrm{Ar}}$ was computed to be more stable than $\mathrm{II}_{\mathrm{Ar}}$, although only by a modest margin of $3.9 \mathrm{kcal} \mathrm{mol}^{-1}$ (Fig. 4).

Scheme 2. FLP-induced synthesis of compounds 3, 4, 5 and 6.

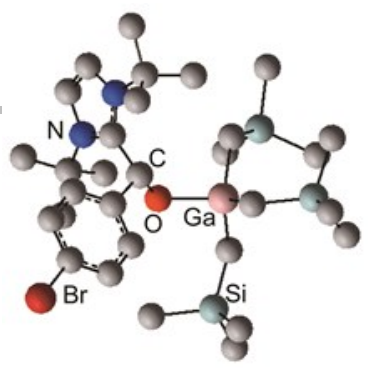

$\left[\mathrm{I}^{\mathrm{B}} \mathrm{BuCH}\left(p-\mathrm{Br}-\mathrm{C}_{6} \mathrm{H}_{4}\right) \mathrm{OGaR}_{3}\right]$ (II $\left.{ }_{\text {Ar }}\right)$

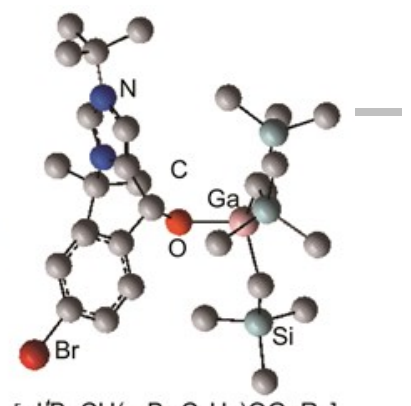

[al'BuCH $\left(p-\mathrm{Br}-\mathrm{C}_{6} \mathrm{H}_{4}\right) \mathrm{OGaR}_{3}$ ] (III ${ }_{\text {Ar }}$ ) 
Figure 4. Modelled structures and relative energies of FLP adducts $\mathrm{II}_{\mathrm{Ar}}$ and $\mathrm{III}_{\mathrm{Ar}}$.

NMR studies (vide supra) suggest that formation of $\mathbf{2}$ takes place via initial coordination of 4-bromobenzaldehyde to Lewis acidic $\mathrm{GaR}_{3}$, which increases the polarity of the $\mathrm{C}=\mathrm{O}$ bond and facilitates its nucleophilic attack by ItBu. Supporting this interpretation, both steps were found to be exothermic, with an overall energy gain of $13.1 \mathrm{kcal} \mathrm{mol}^{-1}$ [Scheme 3(i)]. Contrastingly, illustrating the relevance of $\mathrm{GaR}_{3}$, the reaction of $\mathrm{I}^{\mathrm{t}} \mathrm{Bu}$ with 4bromobenzaldehyde was found to be endothermic by $12.4 \mathrm{kcal}$ $\mathrm{mol}^{-1}$, which is consistent with lack of reactivity witnessed experimentally. The activation of the aldehyde by forming a donoracceptor intermediate is reminiscent to that proposed by Tamm for the fixation of $\mathrm{CO}_{2}$ and $\mathrm{N}_{2} \mathrm{O}$ by $\mathrm{NHC} / \mathrm{B}\left(\mathrm{C}_{6} \mathrm{~F}_{5}\right)_{3} \quad \mathrm{FLP}$ combinations. ${ }^{[23]}$

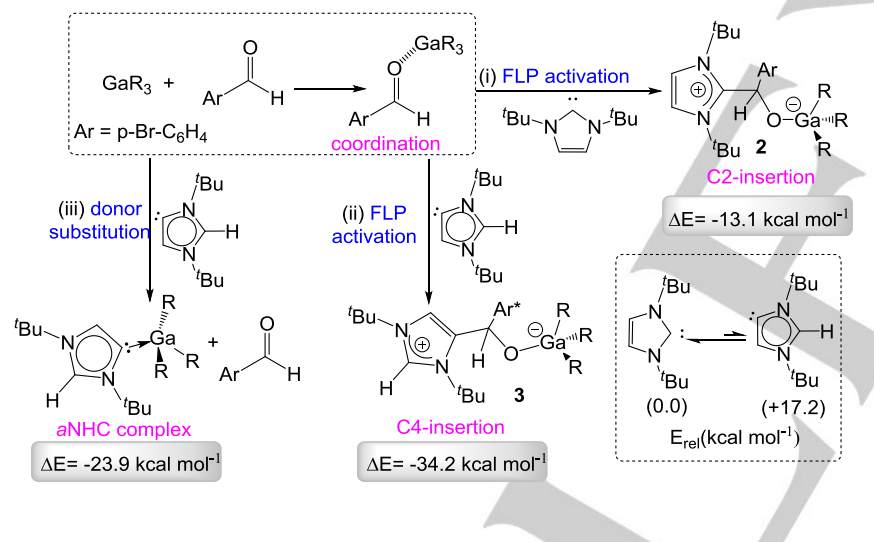

Scheme 3. Proposed scenarios for the reaction of 4-bromobenzaldehylde with $\mathrm{GaR}_{3} / \mathrm{I}^{\mathrm{B}} \mathrm{Bu}$ combinations.

Formation of C4-substituted 3 could happen via a similar pathway, although in this case, abnormal al ${ }^{t} B u$ should act as the LB in the activation process [Scheme 3(ii)]. Having just one $\mathrm{N}$-atom adjacent to their carbenic position, abnormal NHCs are significantly less stabilised than their normal isomers as well as being significantly better donors. ${ }^{[21]}$ Although usually considered as transient species, Bertrand has succeeded in the isolation of a stable free aNHC derived from a 1,2,3,4-tetraarylated imidazolium salt. ${ }^{[21 a]}$ The computed energy difference between free al ${ }^{t} \mathrm{Bu}$ and $\mathrm{I}^{t} \mathrm{Bu}$ is $+17.2 \mathrm{kcalmol}^{-1} .{ }^{[27]}$ Assuming a fast $\mathrm{I}^{t} \mathrm{Bu} / \mathrm{al} \mathrm{I}^{t} \mathrm{Bu}$ isomerisation equilibrium, ${ }^{[28]}$ as soon as more nucleophilic al ${ }^{\mathrm{B}} \mathrm{Bu}$ is formed in solution (even as a minor product in comparison to $I^{t} \mathrm{Bu}$ ), it can react with the aldehyde $\cdot \mathrm{GaR}_{3}$ donor-acceptor complex furnishing 3 [Scheme 3(ii)]. This C4-insertion is found to be significantly more energetically favoured (by $10.3 \mathrm{kcal} \mathrm{mol}^{-1}$ ) than the competitive formation of abnormal complex [(alt $\left.\left.\mathrm{Bu}) \mathrm{GaR}_{3}\right)\right]$, resulting from substitution of 4-bromobenzaldehyde as a donor to the $\mathrm{GaR}_{3}$ fragment [Scheme 3 (iii)]. Nevertheless, a plausible alternative reaction pathway for the formation of 3 could be the insertion of 4-bromobenzaldehyde into this aNHC Ga complex. This possibility was investigated experimentally by dissolving isolated crystals of $\left.\left[\left(\mathrm{al}^{t} \mathrm{Bu}\right) \mathrm{GaR}_{3}\right)\right]$ and the aldehyde $\mathrm{ArCHO}$ in $d_{8^{-}}$ THF. ${ }^{1} \mathrm{H}$ NMR monitoring of the reaction mixture showed that at room temperature, after 2 days no reaction was observed between the reagents, which suggests that $\left.\left[\left(a l^{t} \mathrm{Bu}\right) \mathrm{GaR}_{3}\right)\right]$ is actually a deactivation product and once it is formed, any possible FLP reactivity with unsaturated substrates is inhibited. This is in line with previous studies in other related FLP systems containing $\mathrm{B}$ or Al LAs for which it has been reported that the relevant abnormal NHC complexes fail to exhibit FLP reactivity. ${ }^{[4,9 a]}$

The lability of complex $\mathbf{2}$, which is in dynamic equilibrium with its free components, is also key in promoting the formation of $\mathbf{3}$, as it enables over time the $I^{t} \mathrm{Bu} / \mathrm{al} \mathrm{I}^{t} \mathrm{Bu}$ isomerisation. Thus complex $\mathbf{1}$, resulting from the formal $\mathrm{C} 2$-insertion of formaldehyde, displays no signs of dissociation in solutions over prolonged period of time and does not seem to readily form the relevant $\mathrm{C} 4$-insertion isomer. Offering further support to these experimental findings, DFT calculations on the optimised structures $\left[{ }^{t} \mathrm{BuCH}_{2} \mathrm{OGaR}_{3}\right]$ $\left(\mathrm{II}_{\mathrm{H}}\right)$ and $\left[\mathrm{al} \mathrm{B}^{\mathrm{B} u C H} \mathrm{H}_{2} \mathrm{OGaR}_{3}\left(\mathrm{III}_{\mathrm{H}}\right)\right.$, showed that in this case there is not a clear thermodynamic drive for the $\mathrm{C} 2 / \mathrm{C} 4$ isomerization (see $\mathrm{SI}$ for details). Both models have almost identical energies ( $\mathrm{III}_{\mathrm{H}}$ being just $0.2 \mathrm{kcalmol}^{-1}$ more stable than $\mathrm{I}_{\mathrm{H}}$ ). The small size of the reduced aldehyde in 1 may also contribute to the lack of lability observed for this complex. In this regard, dissociation energy of model $\mathrm{II}_{\mathrm{H}}$ into $\mathrm{I}^{\mathrm{t}} \mathrm{Bu}, \mathrm{GaR}_{3}$ and $\mathrm{CH}_{2} \mathrm{O}$ was found to be $+31.7 \mathrm{kcal}$ $\mathrm{mol}^{-1}\left(18.6 \mathrm{kcal} \mathrm{mol}^{-1}\right.$ greater than for $\left.\|_{\mathrm{A}_{\mathrm{r}}}\right)$.

Reactions with ketones: $\mathbf{C} 4$ insertion vs $\mathbf{C}-\mathrm{H}$ activation

We next turned our attention to the reactivity of these $\mathrm{NHC/Ga}$ combinations towards ketones (Scheme 4).

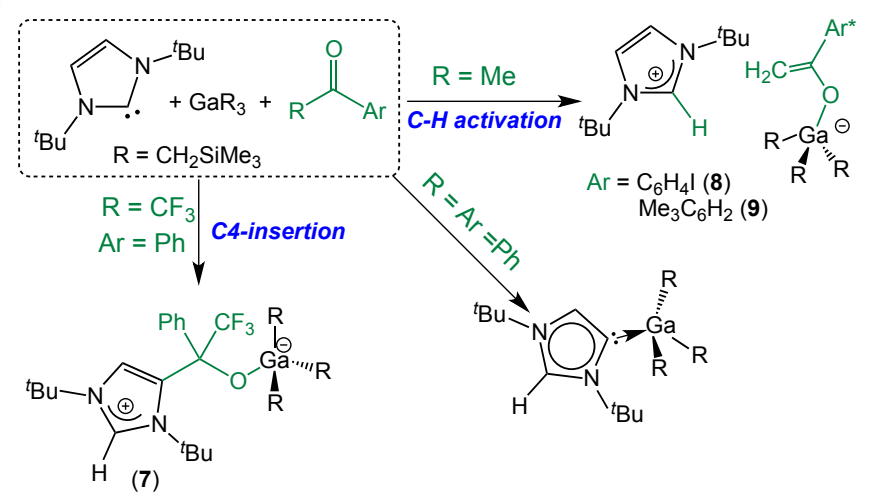

Scheme 4. Contrasting reactivities of $I^{t} \mathrm{Bu} / \mathrm{GaR}_{3}$ mixtures with ketones.

Initial studies using benzophenone led to the formation of the deactivation complex $\left.\left[\left(\mathrm{al}^{\mathrm{t}} \mathrm{Bu}\right) \mathrm{GaR}_{3}\right)\right]$. Trying to circumvent this 
problem, we next probed the same reaction using the saturated version of ItBu, namely bis(tert-butyl)imidazolin-2-ylidene (SItBu). However even under forcing reaction conditions $\left(60^{\circ} \mathrm{C}, 12 \mathrm{~h}\right)$, formation of an insertion product could not be detected (see $\mathrm{SI}$ ). Moving to the more electrophilic $\alpha, \alpha, \alpha$-trifluoroacetophenone, containing the strong electron withdrawing $\mathrm{CF}_{3}$ group, furnished [alt $\mathrm{BuC}(\mathrm{Ph})\left(\mathrm{CF}_{3}\right) \mathrm{OGaR}_{3}$ ] (7) in a $63 \%$ isolated crystalline yield (Scheme 4). Established by X-ray crystallographic studies, the molecular structure of 7 is reminiscent to that of 3 (Fig. 5), revealing that the $I^{t} \mathrm{Bu}$ has added across the $\mathrm{C}=\mathrm{O}$ group of the ketone by its abnormal C4-position [i.e., C2 in Fig. 5]. Multinuclear NMR studies are consistent with the retention of the solid-state structure of 7 in $d_{8}$-THF solution. As observed for compounds 3 6, two singlets show up in the ${ }^{1} \mathrm{H}$ NMR spectrum at 8.67 and 7.21 ppm for the imidazole hydrogen atoms, which is consistent with the lack of symmetry in the heterocyclic ring. ${ }^{13} \mathrm{C}$ NMR spectrum showed an informative resonance at $81.7 \mathrm{ppm}$ belonging to the reduced $\mathrm{C}=\mathrm{O}$ group (which resonates at $191.2 \mathrm{ppm}$ in the starting material).

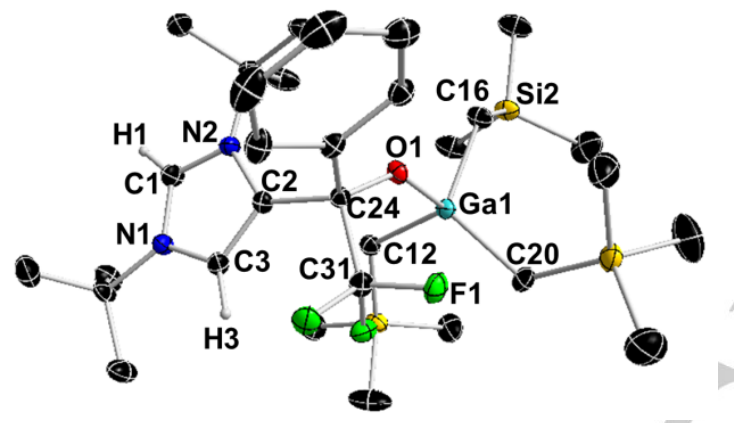

Figure 5. Molecular structure of 7 with $50 \%$ probability displacement ellipsoids All hydrogen atoms except those on the imidazole ring are omitted for clarity. Selected bond distances $(\AA)$ and bond angles $\left({ }^{\circ}\right)$ : $\mathrm{Ga}(1)-\mathrm{O}(1)$ 1.9692(14), $\mathrm{Ga}(1)-\mathrm{C}(12)$ 2.022(2), Ga(1)-C(16) 2.012(2), Ga(1)-C(20) 2.010(2), O(1)-C(24) 1.361(2), C(2)-C(24) 1.544(3), O(1)-Ga(1)-C(20) 107.81(9), O(1)-Ga(1)-C(12) 104.63(8), C(20)-Ga(1)-C(12) 116.46(9), O(1)-Ga(1)-C(16) 96.37(8), C(20)$\mathrm{Ga}(1)-\mathrm{C}(16) \quad 115.00(10), \quad \mathrm{C}(12)-\mathrm{Ga}(1)-\mathrm{C}(16) \quad 113.70(10), \quad \mathrm{N}(1)-\mathrm{C}(1)-\mathrm{N}(2)$ 110.93(18).

Contrasting with the reactivity studies on aldehydes, ${ }^{1} \mathrm{H}$ NMR monitoring of the reaction of $\mathrm{I}^{\mathrm{t}} \mathrm{Bu}, \mathrm{GaR}_{3}$ and $\mathrm{PhC}(\mathrm{O}) \mathrm{CF}_{3}$ did not detect the initial formation of a $\mathrm{C} 2$ insertion isomer or an equilibrium between the starting materials and adduct 7. A plausible explanation may lie in the large steric hindrance of electron withdrawing $\mathrm{CF}_{3}$, which has been described as intermediate in size between ${ }^{i} \mathrm{Pr}$ and ${ }^{t} \mathrm{Bu}$ substituents, ${ }^{[29]}$ which may make difficult the approach of normal I'Bu to the electrophilic $\mathrm{C}=\mathrm{O}$ group of the substrate. The reduced steric congestion on $a^{t} \mathrm{Bu}$, having just one $\mathrm{N}^{t} \mathrm{Bu}$-fragment adjacent to its carbenic position, seems to be sufficient to promote instead formation of C4-insertion adduct 7. Further support was found by DFT calculations, where the reaction of $\mathrm{I}^{t} \mathrm{Bu}, \mathrm{GaR}_{3}$ and $\mathrm{PhC}(\mathrm{O}) \mathrm{CF}_{3}$ to give [al' ${ }^{\mathrm{B} u C}(\mathrm{Ph})\left(\mathrm{CF}_{3}\right) \mathrm{OGaR}_{3}$ ] (IIIlCF3) was calculated to be exergonic by $14.5 \mathrm{kcal} \mathrm{mol}^{-1}$. Geometrical parameters of the optimised structure IIICF3 (Fig. 6) are in excellent agreement with those found experimentally by X-ray analysis of 7 (see Table S4,
SI). Contrastingly, attempts to optimise the structure of the C2insertion product analogous to $\mathrm{II}_{\mathrm{Ar}}$ and $\mathrm{II}_{\mathrm{H}}$, led consistently to a structure where the normal carbenic carbon of the NHC is located $5.708 \AA$ from the $\mathrm{C}=\mathrm{O}$ group of the ketone, evidencing the steric incompatibility of the reagents to promote a $\mathrm{C} 2$ addition process (IlcF3 in Fig. 6). It should be noted that this model was found to be $12.4 \mathrm{kcal} \mathrm{mol}^{-1}$ less stable than IIIcF3.

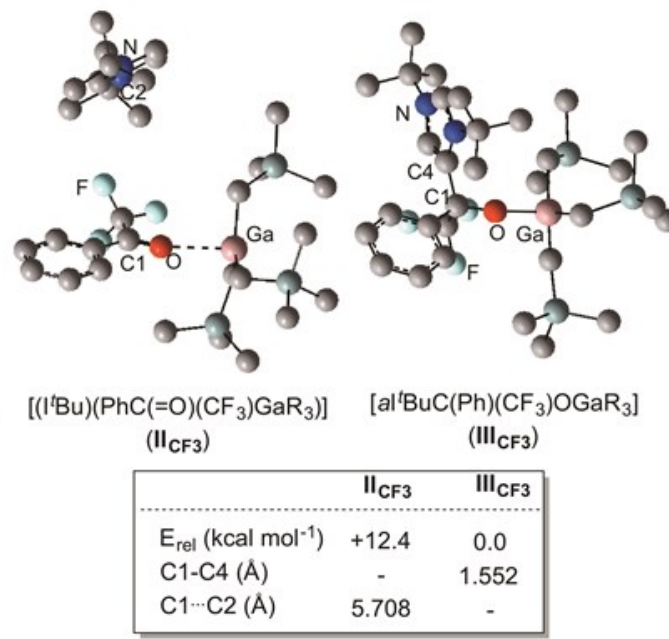

Figure 6. Modelled structures and relative energies of FLP adducts II $\mathrm{CF}_{3}$ and IIICF3.

Interestingly, the reactions of $\mathrm{GaR}_{3} / \mathrm{I}^{t} \mathrm{Bu}$ with enolizable ketones 4-iodoacetophenone or 2,4,6-trimethylacetophenone revealed a different FLP reactivity pattern, promoting in this case the $\mathrm{C}-\mathrm{H}$ activation processes to yield mixed imidazolium gallate salts $\left[\left\{\mathrm{I}^{\mathrm{B} u H}\right\}^{+}\left\{\left(\mathrm{p}-\mathrm{I}-\mathrm{C}_{6} \mathrm{H}_{4}\right) \mathrm{C}\left(\mathrm{CH}_{2}\right) \mathrm{OGaR}_{3}\right\}^{-}\right]$

and $\left[\left\{I^{t} B u H\right\}^{+}\left\{\left(\mathrm{Me}_{3} \mathrm{C}_{6} \mathrm{H}_{2}\right) \mathrm{C}\left(\mathrm{CH}_{2}\right) \mathrm{OGaR}_{3}\right\}\right]$ (9) (Scheme 4). Analogous reactivity was observed when using $\mathrm{SI}^{t} \mathrm{Bu}$, leading to the chemoselective formation of $\left[\left\{S I^{t} \mathrm{BuH}\right\}^{+}\{(\mathrm{p}-\mathrm{I}-\right.$ $\left.\left.\left.\mathrm{C}_{6} \mathrm{H}_{4}\right) \mathrm{C}\left(\mathrm{CH}_{2}\right) \mathrm{OGaR}_{3}\right\}^{-}\right]$

and $\left[\left\{\mathrm{SI} I^{t} \mathrm{BuH}\right\}^{+}\left\{\left(\mathrm{Me}_{3} \mathrm{C}_{6} \mathrm{H}_{2}\right) \mathrm{C}\left(\mathrm{CH}_{2}\right) \mathrm{OGaR}_{3}\right\}^{-}\right] \quad$ (11) (isolated yields ranging from 60 to $84 \%$, see SI for details). The low solubility of these compounds in hexane precluded their isolation as single crystals. NMR spectroscopic studies confirmed the presence of an imidazolium cation [singlets at 8.72 and $7.78 \mathrm{ppm}$ for the imidazole protons integrating one and two protons respectively in the ${ }^{1} \mathrm{H}$ NMR spectrum] as well as the formation of an enolate anion [olefinic $\mathrm{Hs}$ resonate at 3.86 and $4.12 \mathrm{ppm}$ whilst the $\mathrm{C}=\mathrm{CH}_{2}$ fragment gives rise to two resonances at 163.3 and $79.9 \mathrm{ppm}$ in the ${ }^{13} \mathrm{C}$ NMR spectrum].

The isolation of 8-10 uncovers the ability of these $\mathrm{NHC} / \mathrm{Ga}$ systems for the activation of acidic $\mathrm{C}-\mathrm{H}$ bonds. Evidencing their cooperative behaviour, neither the carbenes employed nor $\mathrm{GaR}_{3}$ can deprotonate these substrates on their own. This approach can be extended to other unsaturated organic substrates containing $\alpha$-acidic protons.

Thus $I^{t} \mathrm{Bu} / \mathrm{GaR}_{3}$ readily activates diphenylacetonitrile, affording novel $\left[\left\{{ }^{t} \mathrm{BuH}\right\}^{+}\left\{\mathrm{Ph}_{2} \mathrm{C}=\mathrm{C}=\mathrm{NGaR}_{3}\right\}^{-}\right](12)$ in $64 \%$ yield (Scheme 5), with $I^{t} \mathrm{Bu}$ abstracting the $\alpha$-proton of the substrate while the alkyl groups on $\mathrm{Ga}$ are mere spectators in the reaction. ${ }^{[30]}$ This 
reactivity contrasts with that observed by Mitzel for the reaction of 4-ethynyl-2,6-lutidine with $\mathrm{GaEt}_{3}$, where metallation of the ethynyl group is observed along with elimination of ethane. ${ }^{[10]}$ Replacing $\mathrm{I}^{t} \mathrm{Bu}$ by $\mathrm{SI} \mathrm{Bu}$ furnished the analogous compound $\left.\left[\mathrm{SI} I^{t} \mathrm{BuH}\right\}^{+}\left\{\mathrm{Ph}_{2} \mathrm{C}=\mathrm{C}=\mathrm{NGaR}\right\}^{-}\right](13)$.

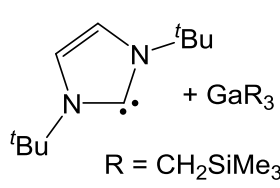<smiles>CC(C#N)c1ccccc1</smiles><smiles>[R][Ge]([R])([R])N=C=C(c1ccccc1)c1cccn1C(C)(C)C</smiles>

(12)
Scheme 5. Synthesis of compound 12 by $\mathrm{C}-\mathrm{H}$ activation of dipehenylacetonitrile employing a $\mathrm{GaR}_{3} / \mathrm{I}^{\mathrm{t}} \mathrm{Bu}$ combinations.

Elucidated by a single crystal $\mathrm{X}$-ray diffraction analysis, the molecular structure of $\mathbf{1 2}$ shows a salt-like ion-pair structure comprised of a protonated imidazolium cation charge-balanced by a heteroleptic gallate anion containing a 3:1 ratio of monosilyl groups to keteniminate ligands (Fig. 7).

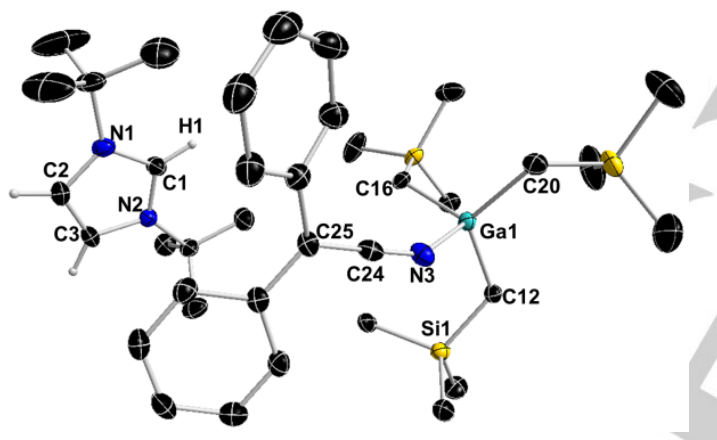

Figure 7. Molecular structure of $\mathbf{1 2}$ with $50 \%$ probability displacement ellipsoids. All $\mathrm{H}$ atoms except those on the imidazole ring and minor disorder in one of the two ${ }^{t} \mathrm{Bu}$ substituents are omitted for clarity. Selected bond distances $(\AA)$ and bond angles $\left({ }^{\circ}\right)$ : $\mathrm{Ga}(1)-\mathrm{N}(3)$ 2.0726(19), $\mathrm{Ga}(1)-\mathrm{C}(12) 2.000(2), \mathrm{Ga}(1)-\mathrm{C}(16)$ 1.988(2), $\mathrm{Ga}(1)-\mathrm{C}(20)$ 1.999(3), N(3)-C(24) 1.171(3), C(24)-C(25) 1.378(3), $\mathrm{N}(3)-\mathrm{Ga}(1)-\mathrm{C}(20)$ 101.08(9), N(3)-Ga(1)-C(12) 100.57(9), C(20)-Ga(1)-C(12) 113.75(11), N(3)-Ga(1)-C(16) 103.06(9), C(20)-Ga(1)-C(16) 117.63(10), C(12)$\mathrm{Ga}(1)-\mathrm{C}(16) 116.82(10), \mathrm{N}(1)-\mathrm{C}(1)-\mathrm{N}(2) 109.7(2)$.

The heteroallene nature of the keteniminate ligand is evident from the shortening of the $\mathrm{C} 24-\mathrm{C} 25$ bond $(1.378 \AA)$ and elongation of the N3-C24 bond (1.171 $\AA$ ) compared to the bonds in the free nitrile $\mathrm{Ph}_{2} \mathrm{CHCN}$ (1.470 (2) $\AA$ and 1.147(2) $\AA$, respectively). ${ }^{[31]}$ These bond distances as well as the near linearity of the C25C24-N3 angle $\left(175.26^{\circ}\right)$ are in close agreement with other structurally characterised metal-keteniminate complexes. ${ }^{[31,32]}$ As far as we can ascertain, this is the first example of a structurally characterised Ga-keteniminate complex. Its ${ }^{1} \mathrm{H}$ NMR spectrum in $\mathrm{d}_{8}$-THF displays three singlets for the imidazolium cation at 8.68 , 7.59 and $1.56 \mathrm{ppm}$ for the $\mathrm{N}_{2} \mathrm{CH}, \mathrm{NCH}$ and ${ }^{t} \mathrm{Bu}$ groups respectively. In agreement with previous literature values, the newly formed ketenimine ligand gives rise to two low intensity resonances in the ${ }^{13} \mathrm{C}$ NMR spectrum, at 55.7 and $143.4 \mathrm{ppm}$ for the $\mathrm{Ph}_{2} \mathrm{C}$ and $\mathrm{C}=\mathrm{N}$ groups respectively. The $v(\mathrm{C}=\mathrm{C}=\mathrm{N})$ stretch, which is the most characteristic vibrational mode in the IR spectrum for the ketenimine ligand, is visible at $2073.5 \mathrm{~cm}^{-1}$. ${ }^{\text {[32] }}$

\section{Conclusions}

The bulky trisalkylgallium, $\mathrm{Ga}\left(\mathrm{CH}_{2} \mathrm{SiMe}_{3}\right)_{3}$, has been found to be a viable, effective Lewis acid for promoting small molecule activation when combined with a strong base such as 1,3-bis(tertbutyl)imidazol-2-ylidene ( $\left.I^{t} \mathrm{Bu}\right)$ thus further expanding the versatility of Frustrated Lewis pair chemistry. The activation of carbonyl compounds can be achieved either by reduction of the $\mathrm{C}=\mathrm{O}$ functionality affording zwitterionic compounds such as [It $\left.\mathrm{BuCH}_{2} \mathrm{OGaR}_{3}\right](1)$ by forming a new $\mathrm{C}-\mathrm{C}$ bond, or by $\mathrm{C}-\mathrm{H}$ bond activation yielding $\left[\left\{I^{t} \mathrm{BuH}\right\}^{+}\left\{\left(\mathrm{Me}_{3} \mathrm{C}_{6} \mathrm{H}_{2}\right) \mathrm{C}\left(\mathrm{CH}_{2}\right) \mathrm{OGaR}_{3}\right\}^{-}\right]$(9), for instance. The unprecedented isolation of conformational isomers $\left[{ }^{t} \mathrm{BuCH}\left(p-\mathrm{Br}-\mathrm{C}_{6} \mathrm{H}_{4}\right) \mathrm{OGaR}_{3}\right.$ ] (2) and [al' $\mathrm{BuCH}\left(p-\mathrm{Br}-\mathrm{C}_{6} \mathrm{H}_{4}\right) \mathrm{OGaR}_{3}$ ] (3), resulting from the activation of 4-bromobenzaldehyde by this novel FLP system has revealed that ItBu can effectively act as a Lewis base not only via its normal $\mathrm{C} 2$ position (kinetic product) but also via its C4 (abnormal) site (thermodynamic product). Evidencing the synergistic behaviour of these $\mathrm{NHC} / \mathrm{GaR}_{3}$ pairings, when separated none of the components is able to activate these carbonyl compounds.

\section{Experimental Section}

Full experimental and computational details and characterization of compounds 1-13 are included in the Supporting Information. CCDC 1492929-1492933 contain the supplementary crystallographic data of this paper. These data can be obtained free of charge from the Cambridge Crystallographic Data Centre via www.ccdc.cam.ac.uk/data request/cif.

Synthesis of [It $\left.\mathrm{BuCH}_{2} \mathrm{OGaR}_{3}\right]$ (1) A solution of $\mathrm{Ga}\left(\mathrm{CH}_{2} \mathrm{SiMe}_{3}\right)_{3}(0.165 \mathrm{~g}$, $0.5 \mathrm{mmol}$ in $10 \mathrm{~mL}$ hexane) was cooled on an ice bath. Paraformaldehyde $(0.015 \mathrm{~g}, 0.5 \mathrm{mmol})$ was then added, followed by the addition of bis(tertbutyl)imidazol-2-ylidene ( $\left.\mathrm{I}^{\mathrm{t}} \mathrm{Bu}\right)(0.09 \mathrm{~g}, 0.5 \mathrm{mmol})$. Obtained white suspension was stirred for $2 \mathrm{~h}$ at $0{ }^{\circ} \mathrm{C}$. Toluene was added $(1 \mathrm{~mL})$ and the mixture was gently heated until all of the visible solid had dissolved. Slow cooling of the resulting solution afforded a crop of colourless crystals (0.18 g, 66\%). Anal. Calcd for $\mathrm{C}_{24} \mathrm{H}_{55} \mathrm{~N}_{2} \mathrm{OSi}{ }_{3} \mathrm{Ga}$ : C, 53.21; $\mathrm{H}, 10.23 ; \mathrm{N}, 5.17$. Found: C, 53.11; H, 10.31; N, 5.39. ${ }^{1} \mathbf{H}$ NMR (298 K, $\boldsymbol{d}_{8}$-THF) $\delta(\mathrm{ppm})-0.82$ $\left(6 \mathrm{H}, \mathrm{s}, \mathrm{CH}_{2} \mathrm{SiMe}_{3}\right),-0.06\left(27 \mathrm{H}, \mathrm{s}, \mathrm{Si}\left(\mathrm{CH}_{3}\right)_{3}\right), 1.81\left(18 \mathrm{H}, \mathrm{s}, \mathrm{C}\left(\mathrm{CH}_{3}\right)_{3}\right), 5.05$ $\left(2 \mathrm{H}, \mathrm{s}, \mathrm{OCH}_{2}\right), 7.50(2 \mathrm{H}, \mathrm{s}$, imidazole backbone $\mathrm{CH}) \cdot{ }^{13} \mathbf{C}\left\{{ }^{1} \mathrm{H}\right\}$ NMR (298 K, $\boldsymbol{d}_{8}$-THF) $\delta(\mathrm{ppm}) 1.3\left(\mathrm{CH}_{2} \mathrm{SiMe}_{3}\right), 3.5\left(\mathrm{Si}\left(\mathrm{CH}_{3}\right)_{3}\right), 31.0\left(\mathrm{C}_{\left.\left(\mathrm{CH}_{3}\right)_{3}\right), 58.6}\right.$ $\left(\mathrm{CH}_{2} \mathrm{O}\right), 63.1\left(\mathrm{C}\left(\mathrm{CH}_{3}\right)_{3}\right), 118.6$ (imidazole backbone $\left.\mathrm{CH}\right), 153.2\left(\mathrm{C}_{\text {imidazole- }}\right.$ $\mathrm{CH}_{2} \mathrm{O}$ ).

Synthesis of $\left[\mathrm{I}^{t} \mathrm{BuCH}\left(p-\mathrm{Br}-\mathrm{C}_{6} \mathrm{H}_{4}\right) \mathrm{OGaR}_{3}\right]$ (2) To a cooled solution of $\mathrm{Ga}\left(\mathrm{CH}_{2} \mathrm{SiMe}_{3}\right)_{3} \quad(0.165 \mathrm{~g}, \quad 0.5 \mathrm{mmol}$ in $10 \mathrm{~mL}$ hexane $)$ 4bromobenzaldehyde (92 $\mathrm{mg}, 0.5 \mathrm{mmol}$ ) was added forming a bright yellow solution. Bis(tert-butyl)imidazol-2-ylidene ( $\left.{ }^{t} \mathrm{Bu}\right)(0.09 \mathrm{~g}, 0.5 \mathrm{mmol})$ was then added and the obtained white suspension was stirred for $15 \mathrm{~min}$ at $\underline{0}{ }^{\circ} \mathbf{C}$. The solvent was exchanged in vacuo for $5 \mathrm{~mL}$ of hexane and $5 \mathrm{~mL}$ of toluene and the resulting solution was placed at $-33^{\circ} \mathrm{C}$ yielding a crop of colourless crystals $(0.145 \mathrm{~g}, 42 \%)$. Anal. Calcd for $\mathrm{C}_{30} \mathrm{H}_{58} \mathrm{~N}_{2} \mathrm{OSi} \mathrm{SaBar}_{3}$ : 
C, 51.72; H, 8.39; N, 4.02. Found: C, 52.33; H, 8.84; N, 4.01. ${ }^{1} \mathrm{H}$ NMR (298 K, $\mathbf{d}_{8}$-THF) $\delta(\mathrm{ppm})-0.81\left(6 \mathrm{H}\right.$, mult, $\left.\mathrm{CH}_{2} \mathrm{SiMe}_{3}\right),-0.06\left(27 \mathrm{H}, \mathrm{s}, \mathrm{Si}\left(\mathrm{CH}_{3}\right)_{3}\right)$, $1.62\left(9 \mathrm{H}, \mathrm{s}, \mathrm{C}\left(\mathrm{CH}_{3}\right)_{3}\right), 1.74\left(9 \mathrm{H}, \mathrm{s}, \mathrm{C}\left(\mathrm{CH}_{3}\right)_{3}\right), 6.79(1 \mathrm{H}, \mathrm{s}, \mathrm{CHO}), 7.29(4 \mathrm{H}$ mult, $\mathrm{Ar}-\mathrm{CH}), 7.76(2 \mathrm{H}, \mathrm{s}$, imidazole backbone $\mathrm{CH}) .{ }^{13} \mathrm{C}\left\{{ }^{1} \mathrm{H}\right\}$ NMR $(298 \mathrm{~K}$ $\mathbf{d}_{8}$-THF) $\delta(\mathrm{ppm}) 2.9\left(\mathrm{CH}_{2} \mathrm{SiMe}_{3}\right), 3.5\left(\mathrm{Si}\left(\mathrm{CH}_{3}\right)_{3}\right), 31.7\left(\mathrm{C}_{\left.\left(\mathrm{CH}_{3}\right)_{3}\right), 31.9}\right.$

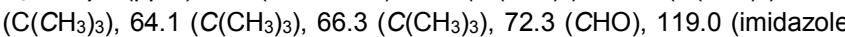
backbone $\mathrm{CH}), 121.8(\mathrm{C}-\mathrm{Br}), 131.7(\mathrm{Ar}-\mathrm{CH}), 133.2(\mathrm{Ar}-\mathrm{CH}), 148.5(\mathrm{Ar}-$ $C_{\text {ipso) }}, 154.2$ ( Cimidazole- $\left.\mathrm{CHO}\right)$.

Synthesis of [alt $\mathrm{BuCH}\left(p-\mathrm{Br}-\mathrm{C}_{6} \mathrm{H}_{4}\right) \mathrm{OGaR}$ ] (3) To a cooled solution of $\mathrm{Ga}\left(\mathrm{CH}_{2} \mathrm{SiMe}_{3}\right)_{3} \quad(0.165 \mathrm{~g}, \quad 0.5 \mathrm{mmol}$ in $10 \mathrm{~mL}$ hexane $)$ 4bromobenzaldehyde $(92 \mathrm{mg}, 0.5 \mathrm{mmol}$ ) was added forming a bright yellow solution. Bis(tert-butyl)imidazol-2-ylidene ( $\left.{ }^{\mathrm{t}} \mathrm{Bu}\right)(0.09 \mathrm{~g}, 0.5 \mathrm{mmol})$ was then added and the obtained white suspension was stirred for $\mathbf{2} \mathrm{h}$ at $0{ }^{\circ} \mathrm{C}$ and concentrated in vacuo to approximately $5 \mathrm{~mL}$ hexane. Toluene was added $(3 \mathrm{~mL})$ and the mixture was gently heated until all of the visible solid had dissolved. Slow cooling of the resulting solution afforded a crop of colourless crystals $(0.20 \mathrm{~g}, 57 \%)$. Anal. Calcd for $\mathrm{C}_{30} \mathrm{H}_{58} \mathrm{~N}_{2} \mathrm{OSi} \mathrm{Si}_{3} \mathrm{GaBr}$ : C, 51.72; H, 8.39; N, 4.02. Found: C, 51.20; H, 8.41; N, 4.23. ${ }^{1} \mathrm{H}$ NMR (298 K, $\boldsymbol{d}_{8}$-THF) $\delta(\mathrm{ppm})-0.92\left(6 \mathrm{H}\right.$, mult, $\left.\mathrm{CH}_{2} \mathrm{SiMe}_{3}\right),-0.10\left(27 \mathrm{H}, \mathrm{s}, \mathrm{Si}\left(\mathrm{CH}_{3}\right)_{3}\right), 1.59$ $\left(9 \mathrm{H}, \mathrm{s}, \mathrm{C}\left(\mathrm{CH}_{3}\right)_{3}\right), 1.69\left(9 \mathrm{H}, \mathrm{s}, \mathrm{C}\left(\mathrm{CH}_{3}\right)_{3}\right), 6.14(1 \mathrm{H}, \mathrm{s}, \mathrm{CHO}), 7.32(4 \mathrm{H}, \mathrm{mult}$ Ar- $\mathrm{CH}), 7.84(1 \mathrm{H}, \mathrm{s}$, imidazole backbone, $\mathrm{CH}), 8.45(1 \mathrm{H}, \mathrm{s}, \mathrm{C} 2-\mathrm{H}) \cdot{ }^{13} \mathrm{C}\left\{{ }^{1} \mathrm{H}\right\}$ NMR $\left(298 \mathrm{~K}, \boldsymbol{d}_{8}\right.$-THF) $\delta(\mathrm{ppm}) 1.9\left(\mathrm{CH}_{2} \mathrm{SiMe}_{3}\right), 3.5\left(\mathrm{Si}\left(\mathrm{CH}_{3}\right)_{3}\right), 29.7$

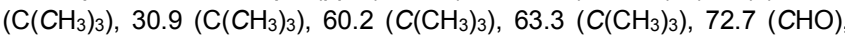
$120.2(\mathrm{C}-\mathrm{Br}), 121.8$ (imidazole backbone $\mathrm{CH}$ ), $129.9(\mathrm{Ar}-\mathrm{CH}), 131.2(\mathrm{Ar}-$ $\mathrm{CH}), 131.8(\mathrm{NCHN}), 146.3$ (Ar- $\left.\mathrm{C}_{\text {ipso }}\right) 149.0$ ( Cimidazole- $\left.\mathrm{CHO}\right)$.

Synthesis of [al'BuC(Ph) $\left(\mathrm{CF}_{3}\right) \mathrm{OGaR}{ }_{3}$ ] (7) A solution of $\mathrm{Ga}\left(\mathrm{CH}_{2} \mathrm{SiMe}_{3}\right)_{3}$ $(0.165 \mathrm{~g}, 0.5 \mathrm{mmol}$ in $10 \mathrm{~mL}$ hexane) was cooled on an ice bath. $\alpha, \alpha, \alpha-$ trifluoroacetophenone $(70 \mu \mathrm{L}, 0.5 \mathrm{mmol})$ was then added, followed by the addition of bis(tert-butyl)imidazol-2-ylidene (ItBu) $(0.09 \mathrm{~g}, 0.5 \mathrm{mmol})$. Obtained white suspension was stirred for $2 \mathrm{~h}$ at $0{ }^{\circ} \mathrm{C}$. Toluene was added $(2 \mathrm{~mL})$ and the mixture was gently heated until all of the visible solid had dissolved. Slow cooling of the resulting solution afforded oil which upon cooling at $-33{ }^{\circ} \mathrm{C}$ afforded a crop of colourless crystals $(0.215 \mathrm{~g}, 63 \%)$. Anal. Calcd for $\mathrm{C}_{31} \mathrm{H}_{58} \mathrm{~F}_{3} \mathrm{~N}_{2} \mathrm{OSSi}_{3} \mathrm{Ga}: \mathrm{C}, 54.29 ; \mathrm{H}, 8.52 ; \mathrm{N}, 4.08$. Found: $\mathrm{C}$, 54.11; H, 7.97; N, 4.56. ${ }^{1} \mathrm{H}$ NMR (298 K, $\boldsymbol{d}_{8}$-THF) $\delta(\mathrm{ppm})-0.83(6 \mathrm{H}, \mathrm{s}$, $\left.\mathrm{CH}_{2} \mathrm{SiMe}_{3}\right),-0.05\left(27 \mathrm{H}, \mathrm{s}, \mathrm{Si}\left(\mathrm{CH}_{3}\right)_{3}\right), 1.51\left(9 \mathrm{H}, \mathrm{s}, \mathrm{C}\left(\mathrm{CH}_{3}\right)_{3}\right), 1.72(9 \mathrm{H}, \mathrm{s}$, $\left.\mathrm{C}\left(\mathrm{CH}_{3}\right)_{3}\right), 7.21(3 \mathrm{H}$, mult, imidazole backbone $\mathrm{CH}+\mathrm{o}-\mathrm{CH}), 7.53(3 \mathrm{H}$, mult, $m-\mathrm{CH}+p-\mathrm{CH}), 8.67(1 \mathrm{H}, \mathrm{s}, \mathrm{C} 2-\mathrm{H}) \cdot{ }^{13} \mathrm{C}\left\{{ }^{1} \mathrm{H}\right\}$ NMR $\left(298 \mathrm{~K}, \boldsymbol{d}_{8}-\mathrm{THF}\right) \delta(\mathrm{ppm})$

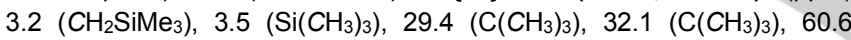
$\left(\mathrm{C}_{\left.\left(\mathrm{CH}_{3}\right)_{3}\right),} 66.6\left(\mathrm{C}\left(\mathrm{CH}_{3}\right)_{3}\right), 81.7 \quad\left(\mathrm{q}, \quad \mathrm{OC}(\mathrm{Ph}) \mathrm{CF}_{3}\right), 119.1\right.$ (imidazole backbone $\mathrm{CH}$ ), $127.6(\mathrm{Ar}-\mathrm{CH}), 128.1(\mathrm{Ar}-\mathrm{CH}), 129.4(\mathrm{Ar}-\mathrm{CH}), 134.1$ $(\mathrm{NCHN}), 141.5$ (Ar- $\left.\mathrm{C}_{\text {ipso }}\right), 145.8\left(\right.$ Cimidazole $\left._{\text {- }} \mathrm{OC}(\mathrm{Ph}) \mathrm{CF}_{3}\right) . \mathrm{CF}_{3}$ could not be confidentially assigned due to the low intensity of its resonances and noise in the baseline. ${ }^{19} \mathrm{~F}$ NMR $\left(298 \mathbf{K}, \boldsymbol{d}_{8}\right.$-THF) $\delta(\mathrm{ppm})-69.92 \mathrm{br} \mathbf{s}$ (additional resonances arising from the decomposition of this sensitive substrate are observed in the ${ }^{19} \mathrm{~F}$ NMR spectrum of both product and starting material).

Synthesis of $\left.\left[\left\{I^{t} \mathrm{BuH}\right\}^{+}\left\{\mathrm{Ph}_{2} \mathrm{C}=\mathrm{C}=\mathrm{NGaR}\right\}_{3}\right\}\right]$ (12) To a cooled solution of $\mathrm{Ga}\left(\mathrm{CH}_{2} \mathrm{SiMe}_{3}\right)_{3}(0.165 \mathrm{~g}, 0.5 \mathrm{mmol}$ in $10 \mathrm{~mL}$ hexane) diphenylacetonitrile $(97 \mathrm{mg}, 0.5 \mathrm{mmol}$ ) was added followed by bis(tert-butyl)imidazol-2-ylidene (I $\mathrm{Bu})(0.09 \mathrm{~g}, 0.5 \mathrm{mmol})$ and the obtained yellow suspension was stirred for $2 \mathrm{~h}$ at $0{ }^{\circ} \mathrm{C}$. Toluene was added $(3 \mathrm{~mL})$ and the mixture was gently heated until all of the visible solid had dissolved. Slow cooling of the resulting solution afforded yellow oil which upon standing provided $\mathrm{X}$-ray quality crystals $(0.225 \mathrm{~g}, 64 \%)$. Anal. Calcd for $\mathrm{C}_{37} \mathrm{H}_{64} \mathrm{GaN}_{3} \mathrm{Si}_{3}$ : C, 63.04; $\mathrm{H}, 9.15 ; \mathrm{N}, 5.96$. Found: $\mathrm{C}, 61.12 ; \mathrm{H}, 8.51 ; \mathrm{N}, 6.04$. The sample consistently shows low values for carbon content. Small amounts of unreacted diphenylacetonitrile are observed in NMR spectra. ${ }^{1} \mathrm{H}$ NMR (298 K, $\boldsymbol{d}_{8}$-THF) $\delta(\mathrm{ppm})-0.69\left(6 \mathrm{H}, \mathrm{s}, \mathrm{CH}_{2} \mathrm{SiMe}_{3}\right),-0.02\left(27 \mathrm{H}, \mathrm{s}, \mathrm{Si}\left(\mathrm{CH}_{3}\right)_{3}\right), 1.56$ $\left(18 \mathrm{H}, \mathrm{s}, \mathrm{C}\left(\mathrm{CH}_{3}\right)_{3}\right), 6.46(2 \mathrm{H}$, mult, $p-\mathrm{CH}), 6.92(4 \mathrm{H}, \mathrm{t}, m-\mathrm{CH}), 7.28(4 \mathrm{H}, \mathrm{t}$, $\mathrm{o}-\mathrm{CH}), 7.59(2 \mathrm{H}$, s, imidazole backbone $\mathrm{CH}), 8.68(1 \mathrm{H}, \mathrm{s}, \mathrm{C} 2-\mathrm{H}) .{ }^{13} \mathrm{C}\left\{{ }^{1} \mathrm{H}\right\}$ NMR (298 K, $\boldsymbol{d}_{8}$-THF) $\delta(\mathrm{ppm}) 2.7\left(\mathrm{CH}_{2} \mathrm{SiMe}_{3}\right), 3.1\left(\mathrm{Si}\left(\mathrm{CH}_{3}\right)_{3}\right), 29.7$
$\left(\mathrm{C}\left(\mathrm{CH}_{3}\right)_{3}\right), 55.7\left(\mathrm{Ph}_{2} \mathrm{C}=\mathrm{C}=\mathrm{N}\right), 61.1 \quad\left(\mathrm{C}_{\left.\left(\mathrm{CH}_{3}\right)_{3}\right),} 117.9(\mathrm{Ar}-\mathrm{CH}), 121.4\right.$ (imidazole backbone $\mathrm{CH}), 123.1(\mathrm{Ar}-\mathrm{CH}), 128.4(\mathrm{Ar}-\mathrm{CH}), 131.9(\mathrm{NCHN})$, 143.4 $\left(\mathrm{Ph}_{2} \mathrm{C}=\mathrm{C}=\mathrm{N}\right), 144.2\left(\mathrm{Ar}-C_{\text {ipso }}\right)$.

\section{Acknowledgements}

We thank the European Research Council (ERC) for their generous sponsorship of this research. We also thank Professor Robert E. Mulvey and $\mathrm{Dr}$ Alberto Hernán-Gómez for their insightful comments, Mr Alexander Clunie for his assistance in the $\mathrm{CHN}$ analysis of these highly air and moisture sensitive compounds and $\mathrm{Mr}$ Craig Irving for his help with NMR spectroscopic experiments.

Keywords: cooperative chemistry $\cdot \mathrm{FLP} \cdot$ small molecule activation $\cdot$ gallium $\cdot$ carbene ligands

[1] G. C. Welch, R. R. S. Juan, J. D. Masuda, D. W. Stephan, Science, 2006 314, 1124-1126.

[2] a) Frustrated Lewis Pairs I: Uncovering and Understanding (Eds.: G Erker, D. W. Stephan), Springer, 2013; b) D. W. Stephan, Dalton Trans. 2009, 3129-3136; c) D. W. Stephan, G. Erker, Angew. Chem. Int. Ed. 2010, 49, 46-76; Angew. Chem. 2010, 122, 50-81; d) D. W. Stephan, Acc Chem. Res., 2015, 48, 306-316; e) D. W. Stephan, G. Erker, Angew. Chem. Int. Ed., 2015, 54, 6400-6441; Angew. Chem. 2015, 127, 64986541; f) D. W. Stephan, J. Am. Chem. Soc. 2015, 137, 10018-10032.

[3] Z. Mo, A. Rit, J. Campos, E. L. Kolychev, S. Aldridge, J. Am. Chem. Soc. 2016, 138, 3306-3309.

[4] a) D. Holschummacher, T. Bannenberg, C. G. Hrib, P. G. Jones, M. Tamm, Angew. Chem. Int. Ed., 2008, 47, 7428-7432; Angew. Chem 2008, 120, 7538-7542; b) D. Holschumacher, C. Taouss, T. Bannenberg, C. G. Hrib, C. G. Daniliuc, P. G. Jones, M. Tamm, Dalton. Trans., 2009 38, 6927-6929; c) D. Holschummacher, T. Bannenberg, K. Ibrom, C. G Daniliuc, P. G. Jones, M. Tamm, Dalton Trans., 2010, 39, 10590-10592 d) S. Kronig, E. Theuergarten, D. Holschumacher, T. Bannenberg, C. G. Daniliuc, P. G. Jones, M. Tamm, Inorg. Chem., 2011, 50, 7344-7359; e) E. L. Kolychev, T. Bannenberg, M. Freytag, C. G. Daniliuc, P. G. Jones M. Tamm, Chem. Eur. J., 2012, 18, 16938-16946; f) E. Theuergarten, J. Schlösser, D. Schlüns, M. Freytag, C. G. Daniliuc, P. G. Jones, M. Tamm, Dalton. Trans., 2012, 41, 9101-9110.

[5] a) P. A. Chase, D. W. Stephan, Angew. Chem. Int. Ed. 2008, 47, 74337437; Angew. Chem. 2008, 120, 7543-7547; b) P. A. Chase, A. L. Gille T. M. Gilbert, D. W. Stephan, Dalton. Trans. 2009, 38, 7179-7188; c) M. A. Dureen, C. C. Brown, D. W. Stephan, Organometallics, 2010, 29 , 6594-6607; d) S. J. Geier, D. W. Stephan, Chem. Commun. 2010, 46 1026-1028; e) M. A. Dureen, G. C. Welch, T. M. Gilbert, D. W. Stephan, Inorg. Chem. 2009, 48, 9910-9917.

[6] a) A. Jana, I. Objartel, H. W. Roesky, D. Stalke, Inorg. Chem. 2009, 48, 7645-7649; b) A. Jana, G. Tavcar, H. W. Roesky, C. Schulzke, Dalton Trans., 2010, 39, 6217-6220; c) J. W. Runyon, O. Steinhof, H. V. R. Dias, J. C. Calabrese, W. J. Marshall, A. J. Arduengo III, Aust. J. Chem., 2011 64, 1165-1172; d) Y. Zhang, G. M. Miyake, E. Y. X. Chen, Angew. Chem Int. Ed. 2010, 49, 10158-10162; Angew. Chem. 2010, 122, 10356-10360 e) B. Ines, S. Holle, R. Goddard, M. Alcarazo, Angew. Chem. Int. Ed. 2010, 49, 8389-8391; Angew. Chem. 2010, 122, 8567-8569.

[7] a) W.-C. Shih, C.-H. Wang, Y.-T. Chang, G. P. A. Yap, T.-G. Ong, Organometallics, 2009, 28, 1060-1067; b) C.-C. Tai, Y.-T. Chang, J.-H. Tsai, T. Jurca, G. P. A. Yap, T.-G. Ong, Organometallics, 2012, 31, $637-$ 643; c) G. Ménard, L. Tran, J. S. J. McCahill, A. J. Lough, D. W. Stephan, Organometallics, 2013, 32, 6759-6763; d) G. Ménard, D. W. Stephan, Angew. Chem. Int. Ed., 2012, 51, 4409-4412; Angew. Chem. 2012, 124, 
4485-4488; e) C. Appelt, H. Westenberg, F. Bertini, A. W. Ehers, J. C Slootweg, K. Lammertsma, W, Uhl, Angew. Chem. Int. Ed. 2011, 50 3925-3928; Angew. Chem. 2011, 123, 4011-4014; f) S. Roters, C. Appelt H. Westenberg, A. Hepp, J. Slootweg, K. Lammertsma, W. Uhl, Dalton Trans. 2012, 41, 9033-9045.

[8] a) T. E. Stennett, J. Pahl, H. S. Zijlstra, F. W. Seidel, S. Harder, Organometallics, 2016, 35, 207-217; b) W. Uhl, C. Appelt Organometallics, 2013, 32, 5008-5014; c) C. Appelt, J. C. Slootweg, K. Lammertsma, W. Uhl, Angew. Chem. Int. Ed, 2013, 52, 4256-4259; Angew. Chem. 2013, 125, 4350-4353; d) C. Appelt, J. C. Slootweg, K. Lammertsma, W. Uhl, Angew. Chem. Int. Ed. 2012, 51, 5911-5914; Angew. Chem. 2012, 124, 6013-6016; e) W. Uhl, C. Appelt, J. Backs, H. Westenberg, A. Wollschlager, J. Tannert, Organometallics, 2014, 33, 1212-1217; f) M. A. Dureen, D. W. Stephan, J. Am. Chem. Soc., 2009 131, 8396-8397; g) M. A. Dureen, C. C. Brown, D. W. Stephan, Organometallics, 2010, 29, 6594-6607; h) G. Ménard, D. W. Stephan, J. Am. Chem. Soc. 2010, 132, 1796-1797; i) G. Ménard, D. W. Stephan Angew. Chem. Int. Ed. 2010, 50, 8396-8399; Angew. Chem. 2011, 123 8546-8549.

[9] a) G. Schnee, O. Nieto Faza, D. Specklin, B. Jacques, L. Karmazin, R. Welter, C. Silva López, S. Dagorne, Chem. Eur. J. 2015, 21, 1795917972; b) G. Schnee, D. Specklin, J.-P. Djukic, S. Dagorne, Organometallics, 2016, 35, 1726-1734.

[10] D. Winkelhaus, B. Neumann, H.-G. Stammler, N. W. Mitzel, Dalton Trans., 2012, 41, 9143-9150.

[11] J. A. B. Abdalla, I. M. Riddlestone, R. Tirfoin, S. Aldridge, Angew. Chem Int. Ed., 2015, 54, 5098-5102; Angew. Chem. 2015, 127, 5187-5191.

[12] W. Uhl, J. Tannert, M. Layh, A. Hepp, S. Grimme, T. Risthaus, Organometallics, 2013, 32, 6770-6779.

[13] C. Ganesamoortht, M. Matthias, D. Bläser, C. Wölper, S. Schulz, Dalton Trans. 2016, 45, 11437-11444.

[14] M. Uzelac, A. Hernán-Gómez, D. R. Armstrong, A. R. Kennedy, E. Hevia, Chem. Sci., 2015, 6, 5719-5728.

[15] a) A. Stute, G. Kehr, C. G. Daniliuc, R. Fröhlich, G. Erker, Dalton Trans. 2013, 42, 4487-4499; b) C. Rosorius, C. G. Daniliuc, R. Fröhlich, G. Kehr G. Erker, J. Organomet. Chem, 2013, 744, 149-155; c) C. Rosorius, G. Kehr, R. Fröhlich, S. Grimme, G. Erker, Organometallics, 2011, 30, 42114219.

[16] M. P. Boone, D. W. Stephan, Organometallics, 2014, 33, 387-393.

[17] a) T. Voss, J.-B. Sortais, R. Fröhlich, G. Kehr, G. Erker, Organometallics, 2011, 30, 584-594; b) S. Schwendemann, R. Fröhlich, G. Kehr, G. Erker, Chem. Sci. 2011, 2, 1842-1849; c) Y. Hasegawa, C. G. Daniliuc, G. Kehr, G. Erker, Angew. Chem. Int. Ed. 2014, 53, 12168-12171; Angew. Chem. 2014, 126, 12364-12367; d) J. Yu, G. Kehr, C. G. Daniliuc, G. Erker, Chem. Commun. 2016, 52, 1393-1396; e) A. T. Normand, C. G. Daniliuc, B. Wibbeling, G. Kehr, P. Le Gendre, G. Erker, Chem. Eur. J, 2016, 22, 4285-4293.

[18] Similarly the Ga-O bond (i.e O1-Ga1 $=1.9317(15) \AA$ ) shows good agreement with literature values for $\mathrm{Ga}$ complexes containing terminal alkoxy ligands: a) P. Horeglad, M. Cybularczyk, B. Trzaskowski, G. Z.
Zukowska, M. Dranka, J. Zachara, Organometallics, 2015, 34, 34803496; b) P. Horeglad, O. Ablialimov, G. Szczepaniak, A. M. Dabrowska, M. Dranka, J. Zachara, Organometallics, 2014, 33, 100-111.

[19] In ${ }^{1} \mathrm{H}$ and ${ }^{13} \mathrm{C}$ NMR spectrum resonances for the adduct of $\mathrm{GaR}_{3}$ and aldehyde are observed, while the resonances for free $\mathrm{I}^{t} \mathrm{Bu}$ are assumed to overlap with the resonances of other species present. However, integration values of the resonances for ${ }^{t} \mathrm{Bu}$ groups reveal that one represents more protons than the other.

[20] From further experiments it is evident that this transformation happens much faster, but in this specific case the ${ }^{1} \mathrm{H}$ NMR spectrum of the same sample was checked the next day.

[21] a) E. Aldeco-Perez, A. J. Rosenthal, B. Donnadieu, P. Parmeswaran, G. Frenking, G. Bertrand, Science, 2009, 326, 556-559; b) J. B. Waters, J. M. Goicoechea, Coord. Chem. Rev. 2015, 293-294, 80-94; c) M. Heckenroth, E. Kluser, A. Neels, M. Albrecht, Angew. Chem. Int. Ed. 2007, 46, 6293-6296; Angew. Chem. 2007, 119, 6409-6412.

[22] a) P. L. Arnold, S. Pearson, Coord. Chem. Rev. 2007, 251, 596-609; b) O. Schuster, L. Yang, H. G. Raubenheimer, M. Albrecht, Chem. Rev. 2009, 109, 3445-3478; c) R. H. Crabtree, Coord. Chem. Rev. 2013, 257, 755-766.

[23] E. Theuergarten, T. Bannenberg, M. D. Walter, D. Holschumacher, M Freytag, C. G. Daniliuc, P. G. Jones, M. Tamm, Dalton. Trans. 2014, 43, 1651-1662.

[24] W. Kohn, A. D. Becke, R. G. Parr, J. Phys. Chem. 1996, 100, 12974 12980.

[25] a) A. D. Becke, Phys. Rev. A, 1988, 38, 3098-3100; b) C. T. Lee, W. T. Yang, R. G. Parr, Phys.Rev. B, 1998, 37, 785-789

[26] a) A. D. McLean, G. S. Chandler, J. Chem. Phys. 1980, 72, 5639-5649 b) R. Krishnan, J. S. Binkley, R. Seeger, J. A. Pople, J. Chem. Phys 1980, 72, 650-655.

[27] The relative stabilities of $\mathrm{I}^{\mathrm{t}} \mathrm{Bu} / \mathrm{al} \mathrm{I}^{\mathrm{B}} \mathrm{B}$ u have previously been computed using a different level of theory (M06/def2-SVP), with $\Delta \mathrm{E}_{\mathrm{a}-\mathrm{n}}=17.7 \mathrm{kcal} \mathrm{mol}^{-1}$ in the gas phase, see reference $9 a$

[28] This has been recently proposed for the isomerisation of [( $\left.\left.\left.{ }^{t} \mathrm{Bu}\right) \mathrm{AlMe}_{3}\right)\right]$ to $\left.\left[a\left(I^{t} \mathrm{Bu}\right) \mathrm{AlMe}_{3}\right)\right]$ see reference $9 \mathrm{a}$

[29] a) I. de Riggi, A. Virgili, M. de Moragas, C. Jaime, J. Org. Chem. 1995, $60,27-31$; b) C. Wolf, W. A. König, C. Roussel, Liebigs Ann. 1995, 781 786.

[30] When the same reaction was carried out with $\mathrm{PhCN}$ no activation process was observed, forming instead abnormal NHC complex altBuGaR3.

[31] E. Iravani, B. Neumüller, Organometallics, 2003, 22, 4129-4135.

[32] a) D. M. Tellers, J. C. M. Ritter, R. G. Bergman, Inorg. Chem. 1999, 38, 4810-4818; b) I. L. Fedushkin, A. G. Morozov, O. V. Rassadin, G. K Fukin, Chem. Eur. J. 2005, 11, 5749-5757; c) J. Zhao, S. Zhang, W.-X Zhang, Z. Xi, Organometallics, 2011, 30, 3464-3467; d) J. Zhao, S Zhang, W.-X. Zhang, Z. Xi, Organometallics, 2011, 31, 8370-8374. 
Entry for the Table of Contents (Please choose one layout)

Layout 1:

\section{FULL PAPER}

\section{Frustrated and gallated!}

By pairing a $\mathrm{Ga}$ tris(alkyl) complex with $\mathrm{N}$-heterocyclic carbene It $\mathrm{Bu}$, three different types of FLP activation processes for carbonyl compounds have been uncovered, namely (i) reduction of $\mathrm{C}=\mathrm{O}$ (via nucleophilic addition of the $\mathrm{NHC}$ using either its $\mathrm{C} 2$
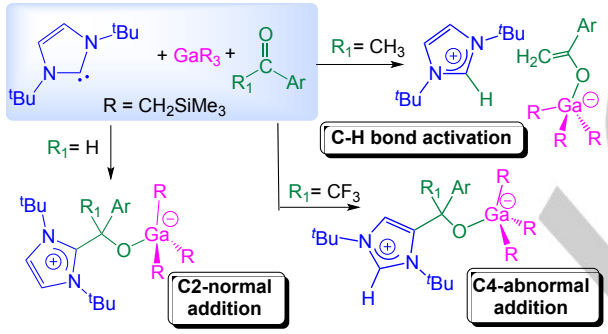

M.Uzelac, D. R. Armstrong, A. R. Kennedy and E. Hevia*

Page No. - Page No.

Understanding the Subtleties of Frustrated Lewis Pair Activation of Carbonyl Compounds by $\mathrm{N}$ -

Heterocyclic Carbene/Alkyl Gallium Pairings

Layout 2:

\section{FULL PAPER}

((Insert TOC Graphic here; max. width: $11.5 \mathrm{~cm}$; max. height: $2.5 \mathrm{~cm})$ )

Author(s), Corresponding Author(s)*

Page No. - Page No.

Title

Text for Table of Contents 


\section{CHEMISTRY A European Journal}

\section{Supporting Information}

Understanding the Subtleties of Frustrated Lewis Pair Activation of Carbonyl Compounds by N-Heterocyclic Carbene/Alkyl Gallium Pairings

Marina Uzelac, David R. Armstrong, Alan R. Kennedy, and Eva Hevia*[a] 


\section{Contents}

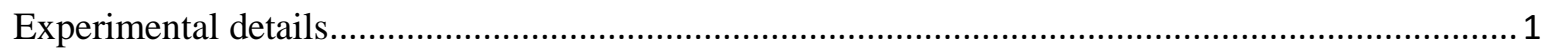

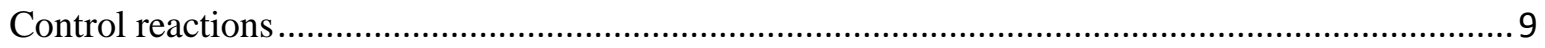

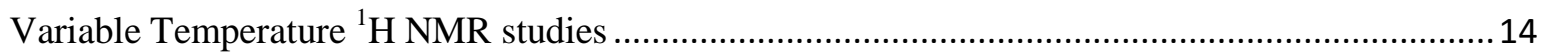

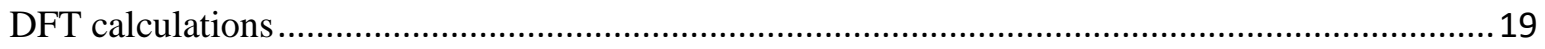

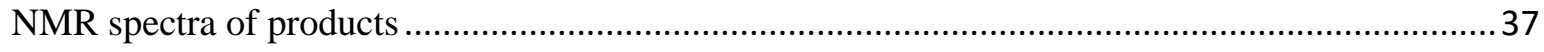

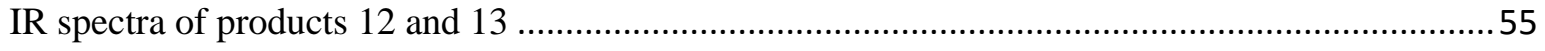

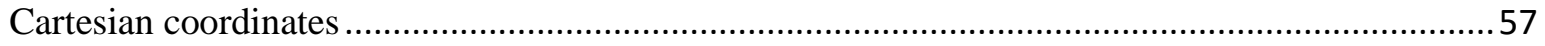

\section{Experimental details}

\section{General}

All reactions were carried out using standard Schlenk and glove box techniques under an inert atmosphere of argon. Solvents (THF, hexane, benzene and toluene) were dried by heating to reflux over sodium benzophenone ketyl and distilled under nitrogen prior to use. NMR spectra were recorded on a Bruker DPX $400 \mathrm{MHz}$ spectrometer, operating at 400.13 $\mathrm{MHz}$ for ${ }^{1} \mathrm{H}$, and $100.62 \mathrm{MHz}$ for ${ }^{13} \mathrm{C}\left\{{ }^{1} \mathrm{H}\right\}$. Elemental analyses were obtained using a Perkin Elmer 2400 elemental analyser. Infrared spectra were obtained as Nujol mulls on $\mathrm{NaCl}$ plates and recorded on a Nicolet 360 FTIR spectrometer spanning range $4000-400 \mathrm{~cm}^{-1}$. Benzaldehyde, 4-bromobenzaldehyde, paraformaldehyde, ferrocenecarboxaldehyde, panisaldehyde, trifluoroacetophenone, 4'-iodoacetophenone, 2',4',6'-trimethylacetophenone, benzophenone, benzonitrile, diphenylacetonitrile were purchased from Sigma Aldrich Chemicals and Alfa Aesar and used as received. All liquid reagents were dried and stored over $4 \AA$ molecular sieves prior to use. $\left[\mathrm{Ga}\left(\mathrm{CH}_{2} \mathrm{SiMe}_{3}\right)_{3}\right],{ }^{1} \mathrm{I}^{t} \mathrm{Bu}^{2}$ and $\mathrm{SI}^{t} \mathrm{Bu}^{3}$ were prepared according to literature methods.

\section{X-Ray Crystallography}

Crystallographic data were measured at 123(2) K for compounds 2, 3, 7, and 12 and at 150(2) $\mathrm{K}$ for compound $\mathbf{1}$ with Oxford Diffraction Gemini S or Xcalibur E instruments and with graphite-monochromated $\mathrm{Mo}(\lambda=0.71073 \AA)$ or $\mathrm{Cu}(\lambda=1.54180 \AA)$ radiation. All structures were refined to convergence on $F^{2}$ using all unique reflections and programs from the

\footnotetext{
${ }^{1}$ L. M. Dennis, W. Patnode, J. Am. Chem. Soc. 1932, 54, 182.

${ }^{2}$ E. C. Hurst, K. Wilson, I. J. Fairlamb, V. Chechik, New J. Chem. 2009, 33, 1837.

${ }^{3}$ (a)K. Arentsen, S. Caddick, F. G. Cloke, Tetrahedron, 2005, 61, 9710; (b) M. K. Denk, A. Thadani, K. Hatano, A. J. Lough Angew. Chem. Int. Ed. 1997, 36, 2607.
} 
SHELX family. ${ }^{4}$ The final model for structure 12 included constraints and restraints on bond lengths and displacement parameters that were required to model disorder for one tert-butyl group. For 3, the Flack parameter refined to -0.002(18). Selected crystallographic data are presented in Table S1 and S2 and full details in cif format can be obtained free of charge from the Cambridge Crystallographic Data Centre via www.ccdc.cam.uk/data_request/cif.

Table S1: Selected crystallographic and refinement parameters for compounds 1-3.

\begin{tabular}{|l|l|l|l|}
\hline \multicolumn{1}{|c|}{ compound } & \multicolumn{2}{c|}{$\mathbf{2}$} & \multicolumn{2}{c|}{ 3 } \\
\hline Empirical formula & $\mathrm{C}_{24} \mathrm{H}_{55} \mathrm{GaN}_{2} \mathrm{OSi}_{3}$ & $\mathrm{C}_{30} \mathrm{H}_{58} \mathrm{BrGaN}_{2} \mathrm{OSi}_{3}$ & $\mathrm{C}_{30} \mathrm{H}_{58} \mathrm{BrGaN}_{2} \mathrm{OSi}_{3}$ \\
\hline Formula weight & 541.69 & 696.68 & 696.68 \\
\hline Crystal system & Triclinic & Monoclinic & Triclinic \\
\hline Space group & $\mathrm{P}-1$ & $\mathrm{P} 2{ }_{1} / \mathrm{c}$ & $\mathrm{P} 1$ \\
\hline$\chi(\AA)$ & 0.71073 & 0.71073 & 1.5418 \\
\hline$a(\AA)$ & $10.9666(6)$ & $9.7662(4)$ & $10.2429(6)$ \\
\hline$b(\AA)$ & $12.2423(7)$ & $20.9419(9)$ & $10.3065(6)$ \\
\hline$c\left(^{\AA}\right)$ & $13.4006(9)$ & $18.5239(8)$ & $10.3660(6)$ \\
\hline$\alpha\left(^{\circ}\right)$ & $99.013(5)$ & 90 & $109.759(5)$ \\
\hline$\beta\left(^{\circ}\right)$ & $101.912(5)$ & $100.234(5)$ & $110.805(6)$ \\
\hline$\gamma\left(^{\circ}\right)$ & $105.054(5)$ & 90 & $97.572(5)$ \\
\hline$V\left(\AA^{3}\right)$ & $1657.33(18)$ & $3728.3(3)$ & $922.44(10)$ \\
\hline$Z$ & 2 & 4 & 1 \\
\hline$\left.\mu(\mathrm{mm})^{-1}\right)$ & 0.955 & 1.929 & 3.392 \\
\hline $2 \theta$ max $\left(^{\circ}\right)$ & 59.98 & 55.00 & 138.43 \\
\hline Measured reflections & 25467 & 33294 & 11818 \\
\hline Unique reflections & 8904 & 8504 & 6187 \\
\hline Observed reflections & 6483 & 6527 & 5348 \\
\hline $\mathrm{R}_{\text {int }}$ & 0.0400 & 0.0449 & 0.0468 \\
\hline $\mathrm{R}[$ on F, obs refln only] & 0.0485 & 0.0378 & 0.0454 \\
\hline wR [on F $\mathrm{F}^{2}$, all data] & 0.1205 & 0.0822 & 0.1086 \\
\hline GoF & 1.035 & 1.036 & 1.038 \\
\hline $\begin{array}{l}\text { Largest diff peak/hole } \\
\left(\mathrm{e} \AA^{-3}\right)\end{array}$ & $1.665 /-0.714$ & $0.731 /-0.380$ & $0.610 /-0.402$ \\
\hline
\end{tabular}

Table S2: Selected crystallographic and refinement parameters for compounds $\mathbf{7}$ and $\mathbf{1 2 .}$

\begin{tabular}{|l|l|l|}
\hline \multicolumn{1}{|c|}{ compound } & \multicolumn{1}{c|}{$\mathbf{7}$} & \multicolumn{1}{c|}{$\mathbf{1 2}$} \\
\hline Empirical formula & $\mathrm{C}_{31} \mathrm{H}_{58} \mathrm{~F}_{3} \mathrm{GaN}_{2} \mathrm{OSi}_{3}$ & $\mathrm{C}_{37} \mathrm{H}_{64} \mathrm{GaN}_{3} \mathrm{Si}_{3}$ \\
\hline Formula weight & 685.78 & 704.90 \\
\hline Crystal system & Monoclinic & Monoclinic \\
\hline Space group & $\mathrm{P} 2{ }_{1} / \mathrm{n}$ & $\mathrm{P} 2{ }_{1} / \mathrm{c}$ \\
\hline$\chi(\AA)$ & 0.71073 & 0.71073 \\
\hline$a(\AA)$ & $9.7056(4)$ & $14.1628(6)$ \\
\hline$b(\AA)$ & $16.5713(7)$ & $18.0691(8)$ \\
\hline$c(\AA)$ & $23.9252(10)$ & $16.5681(8)$ \\
\hline$\alpha\left(^{\circ}\right)$ & 90 & 90 \\
\hline$\beta\left(^{\circ}\right)$ & $96.071(4)$ & $99.490(4)$ \\
\hline$\gamma\left({ }^{\circ}\right)$ & 90 & 90 \\
\hline
\end{tabular}

\footnotetext{
${ }^{4}$ G. M. Sheldrick, Acta Crystallogr., 2008, A64, 112.
} 


\begin{tabular}{|l|l|l|}
\hline$V\left(\AA^{3}\right)$ & $3826.4(3)$ & $4181.9(3)$ \\
\hline$Z$ & 4 & 4 \\
\hline$\mu\left(\mathrm{mm}^{-1}\right)$ & 0.852 & 0.770 \\
\hline $2 \theta \mathrm{max}\left(^{\circ}\right)$ & 59.99 & 59.99 \\
\hline Measured reflections & 113826 & 20370 \\
\hline Unique reflections & 10865 & 10853 \\
\hline Observed reflections & 7577 & 7212 \\
\hline $\mathrm{R}_{\text {int }}$ & 0.0925 & 0.0453 \\
\hline $\mathrm{R}[$ on F, obs refln only] & 0.0438 & 0.0526 \\
\hline wR [on $\mathrm{F}^{2}$ all data] & 0.0990 & 0.1021 \\
\hline GoF & 1.021 & 0.993 \\
\hline $\begin{array}{l}\text { Largest diff peak/hole } \\
\left(\mathrm{e} \AA^{-3}\right)\end{array}$ & $0.587 /-0.379$ & $0.617 /-0.544$ \\
\hline
\end{tabular}

\section{Synthesis of products}

1. Synthesis of $\left[\mathrm{I}^{t} \mathrm{BuCH}_{2} \mathrm{OGaR}_{3}\right](\mathbf{1})$

A solution of $\mathrm{Ga}\left(\mathrm{CH}_{2} \mathrm{SiMe}_{3}\right)_{3}(0.165 \mathrm{~g}, 0.5 \mathrm{mmol}$ in $10 \mathrm{~mL}$ hexane $)$ was cooled on an ice bath. Paraformaldehyde $(0.015 \mathrm{~g}, 0.5 \mathrm{mmol})$ was then added, followed by the addition of bis(tert-butyl)imidazol-2-ylidene $\left(\mathrm{I}^{t} \mathrm{Bu}\right)(0.09 \mathrm{~g}, 0.5 \mathrm{mmol})$. Obtained white suspension was stirred for $2 \mathrm{~h}$ at $0{ }^{\circ} \mathrm{C}$. Toluene was added $(1 \mathrm{~mL})$ and the mixture was gently heated until all of the visible solid had dissolved. Slow cooling of the resulting solution afforded a crop of colourless crystals $(0.18 \mathrm{~g}, 66 \%)$. Anal. Calcd for $\mathrm{C}_{24} \mathrm{H}_{55} \mathrm{~N}_{2} \mathrm{OSi}_{3} \mathrm{Ga}: \mathrm{C}, 53.21 ; \mathrm{H}, 10.23 ; \mathrm{N}$, 5.17. Found: C, 53.11; H, 10.31; N, 5.39.

${ }^{1} \mathbf{H}$ NMR $\left(298 \mathrm{~K}, \boldsymbol{d}_{\mathbf{8}}\right.$-THF) $\delta(\mathrm{ppm})-0.82\left(6 \mathrm{H}, \mathrm{s}, \mathrm{CH}_{2} \mathrm{SiMe}_{3}\right),-0.06\left(27 \mathrm{H}, \mathrm{s}, \mathrm{Si}\left(\mathrm{CH}_{3}\right)_{3}\right), 1.81$ $\left(18 \mathrm{H}, \mathrm{s}, \mathrm{C}\left(\mathrm{CH}_{3}\right)_{3}\right), 5.05\left(2 \mathrm{H}, \mathrm{s}, \mathrm{OCH}_{2}\right), 7.50(2 \mathrm{H}, \mathrm{s}$, imidazole backbone $\mathrm{CH}) .{ }^{13} \mathrm{C}\left\{{ }^{1} \mathbf{H}\right\} \mathbf{N M R}$ $\left(298 \mathrm{~K}, \boldsymbol{d}_{\mathbf{8}}\right.$-THF) $\delta(\mathrm{ppm}) 1.3\left(\mathrm{CH}_{2} \mathrm{SiMe}_{3}\right), 3.5\left(\mathrm{Si}\left(\mathrm{CH}_{3}\right)_{3}\right), 31.0\left(\mathrm{C}\left(\mathrm{CH}_{3}\right)_{3}\right), 58.6\left(\mathrm{CH}_{2} \mathrm{O}\right)$, 63.1 $\left(\mathrm{C}\left(\mathrm{CH}_{3}\right)_{3}\right), 118.6$ (imidazole backbone $\left.\mathrm{CH}\right), 153.2\left(C_{\text {imidazole }}-\mathrm{CH}_{2} \mathrm{O}\right)$.

\section{Synthesis of $\left[\mathrm{I}^{t} \mathrm{BuCH}\left(p-\mathrm{Br}-\mathrm{C}_{6} \mathrm{H}_{4}\right) \mathrm{OGaR}_{3}\right](2)$}

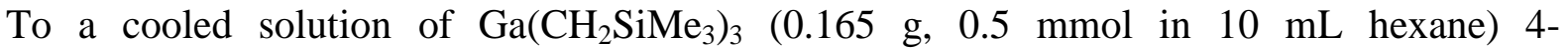
bromobenzaldehyde (92 mg, $0.5 \mathrm{mmol}$ ) was added forming a bright yellow solution. Bis(tertbutyl)imidazol-2-ylidene ( $\left.{ }^{t} \mathrm{Bu}\right)(0.09 \mathrm{~g}, 0.5 \mathrm{mmol})$ was then added and the obtained white

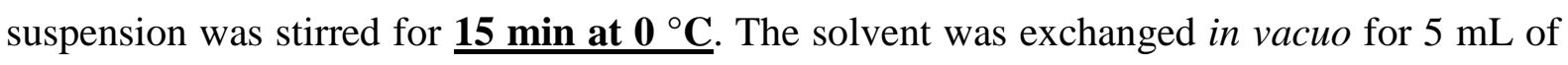
hexane and $5 \mathrm{~mL}$ of toluene and the resulting solution was placed at $-33{ }^{\circ} \mathrm{C}$ yielding a crop of colourless crystals (0.145 g, 42\%). Anal. Calcd for $\mathrm{C}_{30} \mathrm{H}_{58} \mathrm{~N}_{2} \mathrm{OSi}{ }_{3} \mathrm{GaBr}: \mathrm{C}, 51.72 ; \mathrm{H}, 8.39 ; \mathrm{N}$, 4.02. Found: C, 52.33; H, 8.84; N, 4.01. 
${ }^{1} \mathbf{H}$ NMR (298 K, d8 -THF) $\delta(\mathrm{ppm})-0.81\left(6 \mathrm{H}\right.$, mult, $\left.\mathrm{CH}_{2} \mathrm{SiMe}_{3}\right),-0.06\left(27 \mathrm{H}, \mathrm{s}, \mathrm{Si}\left(\mathrm{CH}_{3}\right)_{3}\right)$, $1.62\left(9 \mathrm{H}, \mathrm{s}, \mathrm{C}\left(\mathrm{CH}_{3}\right)_{3}\right), 1.74\left(9 \mathrm{H}, \mathrm{s}, \mathrm{C}\left(\mathrm{CH}_{3}\right)_{3}\right), 6.79(1 \mathrm{H}, \mathrm{s}, \mathrm{CHO}), 7.29$ (4H, mult, Ar- $\left.\mathrm{CH}\right)$, $7.76(2 \mathrm{H}$, s, imidazole backbone $\mathrm{CH}) .{ }^{13} \mathbf{C}\left\{{ }^{1} \mathbf{H}\right\}$ NMR (298 K, d8-THF) $\delta(\mathrm{ppm}) 2.9$ $\left(\mathrm{CH}_{2} \mathrm{SiMe}_{3}\right), 3.5\left(\mathrm{Si}\left(\mathrm{CH}_{3}\right)_{3}\right), 31.7\left(\mathrm{C}_{\left.\left(\mathrm{CH}_{3}\right)_{3}\right),} 31.9\left(\mathrm{C}\left(\mathrm{CH}_{3}\right)_{3}\right), 64.1\left(\mathrm{C}_{\left.\left(\mathrm{CH}_{3}\right)_{3}\right),} 66.3\left(\mathrm{C}\left(\mathrm{CH}_{3}\right)_{3}\right)\right.\right.$, 72.3 (CHO), 119.0 (imidazole backbone $\mathrm{CH}$ ), 121.8 (C-Br), 131.7 (Ar- $\mathrm{CH}), 133.2(\mathrm{Ar}-\mathrm{CH})$, 148.5 (Ar- $\left.C_{i p s o}\right), 154.2\left(C_{\text {imidazole }}-\mathrm{CHO}\right)$.

\section{Synthesis of $\left[a \mathrm{I}^{t} \mathrm{BuCH}\left(p-\mathrm{Br}_{-}-\mathrm{C}_{6} \mathrm{H}_{4}\right) \mathrm{OGaR}_{3}\right](\mathbf{3})$}

To a cooled solution of $\mathrm{Ga}\left(\mathrm{CH}_{2} \mathrm{SiMe}_{3}\right)_{3}(0.165 \mathrm{~g}$, $0.5 \mathrm{mmol}$ in $10 \mathrm{~mL}$ hexane $)$ 4bromobenzaldehyde (92 mg, $0.5 \mathrm{mmol}$ ) was added forming a bright yellow solution. Bis(tertbutyl)imidazol-2-ylidene $\left(\mathrm{I}^{t} \mathrm{Bu}\right)(0.09 \mathrm{~g}, 0.5 \mathrm{mmol})$ was then added and the obtained white suspension was stirred for $\underline{\mathbf{2} \mathbf{h} \text { at } \mathbf{0}}{ }^{\circ} \mathbf{C}$ and concentrated in vacuo to approximately $5 \mathrm{~mL}$ hexane. Toluene was added $(3 \mathrm{~mL})$ and the mixture was gently heated until all of the visible solid had dissolved. Slow cooling of the resulting solution afforded a crop of colourless crystals $(0.20 \mathrm{~g}$, 57\%). Anal. Calcd for $\mathrm{C}_{30} \mathrm{H}_{58} \mathrm{~N}_{2} \mathrm{OSi}_{3} \mathrm{GaBr}$ : C, 51.72; H, 8.39; N, 4.02. Found: C, 51.20; H, $8.41 ; \mathrm{N}, 4.23$.

${ }^{1} \mathbf{H}$ NMR (298 K, $\boldsymbol{d}_{\mathbf{8}}$-THF) $\delta(\mathrm{ppm})-0.92\left(6 \mathrm{H}\right.$, mult, $\left.\mathrm{CH}_{2} \mathrm{SiMe}_{3}\right),-0.10\left(27 \mathrm{H}, \mathrm{s}, \mathrm{Si}\left(\mathrm{CH}_{3}\right)_{3}\right)$, $1.59\left(9 \mathrm{H}, \mathrm{s}, \mathrm{C}\left(\mathrm{CH}_{3}\right)_{3}\right), 1.69\left(9 \mathrm{H}, \mathrm{s}, \mathrm{C}\left(\mathrm{CH}_{3}\right)_{3}\right), 6.14(1 \mathrm{H}, \mathrm{s}, \mathrm{CHO}), 7.32$ (4H, mult, Ar- $\left.\mathrm{CH}\right)$, $7.84(1 \mathrm{H}, \mathrm{s}$, imidazole backbone, $\mathrm{CH}), 8.45(1 \mathrm{H}, \mathrm{s}, \mathrm{C} 2-H) .{ }^{\mathbf{1 3}} \mathbf{C}\left\{{ }^{\mathbf{1}} \mathbf{H}\right\}$ NMR (298 K, $\boldsymbol{d}_{\mathbf{8}}$-THF)

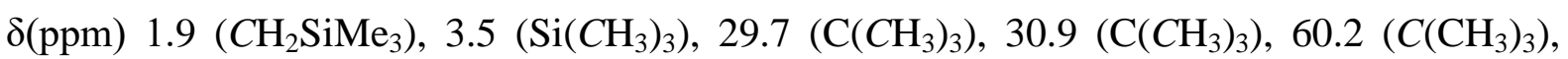
$63.3\left(C\left(\mathrm{CH}_{3}\right)_{3}\right), 72.7(\mathrm{CHO}), 120.2(\mathrm{C}$-Br), 121.8 (imidazole backbone $\mathrm{CH}), 129.9(\mathrm{Ar}-\mathrm{CH})$, $131.2(\mathrm{Ar}-\mathrm{CH}), 131.8(\mathrm{NCHN}), 146.3$ (Ar- $\left.C_{\mathrm{ipso}}\right) 149.0\left(C_{\text {imidazole }}-\mathrm{CHO}\right)$.

4. Synthesis of $\left[a \mathrm{I}^{t} \mathrm{BuCH}\left(\mathrm{C}_{6} \mathrm{H}_{5}\right) \mathrm{OGaR}_{3}\right]$ (4)

A solution of $\mathrm{Ga}\left(\mathrm{CH}_{2} \mathrm{SiMe}_{3}\right)_{3}(0.165 \mathrm{~g}, 0.5 \mathrm{mmol}$ in $10 \mathrm{~mL}$ hexane $)$ was cooled on an ice bath. Benzaldehyde $(51 \mu \mathrm{L}, 0.5 \mathrm{mmol})$ was then added, followed by the addition of bis(tertbutyl)imidazol-2-ylidene ( $\left.{ }^{t} \mathrm{Bu}\right)(0.09 \mathrm{~g}, 0.5 \mathrm{mmol})$. Obtained light yellow suspension was stirred for $2 \mathrm{~h}$ at $0{ }^{\circ} \mathrm{C}$. Toluene was added $(2 \mathrm{~mL})$ and the mixture was gently heated until all of the visible solid had dissolved. Cooling of the resulting solution at $-33{ }^{\circ} \mathrm{C}$ afforded a colourless microcrystalline solid (0.19 g, $62 \%)$. Anal. Calcd for: $\mathrm{C}_{30} \mathrm{H}_{59} \mathrm{~N}_{2} \mathrm{GaOSi}_{3}$ : C, 58.32; H, 9.63; N, 4.53. Found: C, 57.89; H, 9.39; N, 4.24. 
${ }^{1} \mathbf{H}$ NMR (298 K, $\left.\mathbf{C}_{6} \mathbf{D}_{\mathbf{6}}\right) \delta(\mathrm{ppm})-0.32\left(6 \mathrm{H}\right.$, mult, $\left.\mathrm{CH}_{2} \mathrm{SiMe}_{3}\right), 0.46\left(27 \mathrm{H}, \mathrm{s}, \mathrm{Si}\left(\mathrm{CH}_{3}\right)_{3}\right), 0.78$ $\left(9 \mathrm{H}, \mathrm{s}, \mathrm{C}\left(\mathrm{CH}_{3}\right)_{3}\right), 1.06\left(9 \mathrm{H}, \mathrm{s}, \mathrm{C}\left(\mathrm{CH}_{3}\right)_{3}\right), 6.38(2 \mathrm{H}, \mathrm{s}, \mathrm{CHO}), 6.93(1 \mathrm{H}, \mathrm{s}$, imidazole backbone $\mathrm{CH}), 7.09(1 \mathrm{H}, \mathrm{t}, p-\mathrm{CH}), 7.28(2 \mathrm{H}, \mathrm{t}, m-\mathrm{CH}), 7.39(1 \mathrm{H}, \mathrm{s}, \mathrm{C} 2-H), 7.55(2 \mathrm{H}, \mathrm{d}, o-\mathrm{CH})$.

${ }^{1} \mathbf{H}$ NMR (298 K, $\boldsymbol{d}_{\mathbf{8}}$-THF) $\delta(\mathrm{ppm})-0.92\left(6 \mathrm{H}\right.$, mult, $\left.\mathrm{CH}_{2} \mathrm{SiMe}_{3}\right),-0.09\left(27 \mathrm{H}, \mathrm{s}, \mathrm{Si}\left(\mathrm{CH}_{3}\right)_{3}\right)$, $1.56\left(9 \mathrm{H}, \mathrm{s}, \mathrm{C}\left(\mathrm{CH}_{3}\right)_{3}\right), 1.69\left(9 \mathrm{H}, \mathrm{s}, \mathrm{C}\left(\mathrm{CH}_{3}\right)_{3}\right), 6.17(1 \mathrm{H}, \mathrm{s}, \mathrm{OCH}), 7.07(1 \mathrm{H}, \mathrm{t}, p-\mathrm{CH}), 7.16$ $(2 \mathrm{H}, \mathrm{t}, m-\mathrm{CH}), 7.34(2 \mathrm{H}, \mathrm{d}, o-\mathrm{CH}), 7.85(1 \mathrm{H}, \mathrm{s}$, imidazole backbone $\mathrm{CH}), 8.43(1 \mathrm{H}, \mathrm{s}, \mathrm{C} 2-H)$. ${ }^{13} \mathbf{C}\left\{{ }^{1} \mathbf{H}\right\}$ NMR $\left(298 \mathrm{~K}, \boldsymbol{d}_{\mathbf{8}}\right.$-THF) $\delta(\mathrm{ppm}) 1.9\left(\mathrm{CH}_{2} \mathrm{SiMe}_{3}\right), 3.5\left(\mathrm{Si}\left(\mathrm{CH}_{3}\right)_{3}\right), 29.7\left(\mathrm{C}\left(\mathrm{CH}_{3}\right)_{3}\right)$,

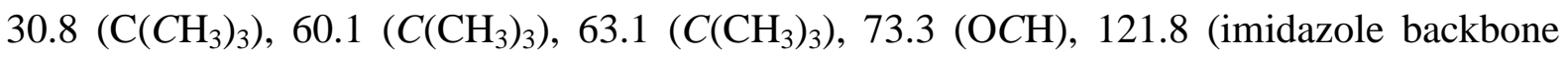
$C \mathrm{H}), 126.5(\mathrm{Ar}-\mathrm{CH}), 128.0(\mathrm{Ar}-\mathrm{CH}), 128.1(\mathrm{Ar}-\mathrm{CH}), 131.4(\mathrm{NCHN}), 147.2\left(\mathrm{Ar}-C_{i p s o}\right) 149.3$ $\left(C_{\text {imidazole }}-\mathrm{OCH}\right)$.

\section{Synthesis of $\left[a I^{t} \mathrm{BuCH}\left(p-\mathrm{OMe}-\mathrm{C}_{6} \mathrm{H}_{4}\right) \mathrm{OGaR}_{3}\right](\mathbf{5})$}

To a cooled solution of $\mathrm{Ga}\left(\mathrm{CH}_{2} \mathrm{SiMe}_{3}\right)_{3}(0.165 \mathrm{~g}, 0.5 \mathrm{mmol}$ in $10 \mathrm{~mL}$ hexane $)$-anisaldehyde $(61 \mu \mathrm{L}, 0.5 \mathrm{mmol})$ was added followed by bis(tert-butyl)imidazol-2-ylidene ( $\left.{ }^{t} \mathrm{Bu}\right)(0.09 \mathrm{~g}$, $0.5 \mathrm{mmol}$ ) and the obtained white suspension was stirred for $2 \mathrm{~h}$ at $0{ }^{\circ} \mathrm{C}$. Toluene was added $(3 \mathrm{~mL})$ and the mixture was gently heated until all of the visible solid had dissolved. Slow cooling of the resulting solution afforded colourless microcrystalline solid $(0.183 \mathrm{~g}, 56 \%)$. Anal. Calcd for $\mathrm{C}_{31} \mathrm{H}_{61} \mathrm{GaN}_{2} \mathrm{O}_{2} \mathrm{Si}_{3}$ : C, 57.48; H, 9.49; N, 4.32. Found: C, 57.65; H, 8.69; N, 4.39.

${ }^{1}$ H NMR (298 K, $\boldsymbol{d}_{\mathbf{8}}$-THF) $\delta(\mathrm{ppm})-0.93\left(6 \mathrm{H}\right.$, mult, $\left.\mathrm{CH}_{2} \mathrm{SiMe}_{3}\right),-0.10\left(27 \mathrm{H}, \mathrm{s}, \mathrm{Si}\left(\mathrm{CH}_{3}\right)_{3}\right)$, $1.68\left(9 \mathrm{H}, \mathrm{s}, \mathrm{C}\left(\mathrm{CH}_{3}\right)_{3}\right), 1.69\left(9 \mathrm{H}, \mathrm{s}, \mathrm{C}\left(\mathrm{CH}_{3}\right)_{3}\right), 3.70\left(1 \mathrm{H}, \mathrm{s}, \mathrm{OCH}_{3}\right), 6.12(1 \mathrm{H}, \mathrm{s}, \mathrm{CHO}), 6.72$ $(2 \mathrm{H}, \mathrm{d}, \mathrm{Ar}-\mathrm{CH}), 7.22(2 \mathrm{H}, \mathrm{d}, \mathrm{Ar}-\mathrm{CH}), 7.83(1 \mathrm{H}, \mathrm{s}$, imidazole backbone $\mathrm{CH}), 8.41(1 \mathrm{H}, \mathrm{s}, \mathrm{C} 2-$ $H),{ }^{13} \mathbf{C}\left\{{ }^{1} \mathbf{H}\right\}$ NMR $\left(298 \mathbf{~ K}, d_{\mathbf{8}}\right.$-THF) $\left.\delta(\mathrm{ppm}) 1.9\left(\mathrm{CH}_{2} \mathrm{SiMe}_{3}\right), 3.6\left(\mathrm{Si}\left(\mathrm{CH}_{3}\right)_{3}\right), 29.7\left(\mathrm{C} \mathrm{CH}_{3}\right)_{3}\right)$, $30.8\left(\mathrm{C}\left(\mathrm{CH}_{3}\right)_{3}\right), 55.2\left(\mathrm{OCH}_{3}\right), 60.0\left(\mathrm{C}_{\left.\left(\mathrm{CH}_{3}\right)_{3}\right),} 63.0\left(\mathrm{C}\left(\mathrm{CH}_{3}\right)_{3}\right), 72.9(\mathrm{OCH}), 113.6(\mathrm{Ar}-\mathrm{CH})\right.$, 121.7 (imidazole backbone $\mathrm{CH}), 129.0(\mathrm{Ar}-\mathrm{CH}), 131.3(\mathrm{NCHN}), 141.3\left(\mathrm{Ar}-C_{i p s o}\right)$ 147.7( $\left.C_{\text {imidazole }}-\mathrm{OCH}\right), 159.1$ (C-OMe).

\section{Synthesis of $\left[a \mathrm{I}^{t} \mathrm{BuCH}\left(\mathrm{FeCp}_{2}\right) \mathrm{OGaR}_{3}\right](\mathbf{6})$}

To a cooled solution of $\mathrm{Ga}_{\left(\mathrm{CH}_{2} \mathrm{SiMe}_{3}\right)_{3}} \quad(0.165 \mathrm{~g}, 0.5 \mathrm{mmol}$ in $10 \mathrm{~mL}$ hexane $)$ ferrocenecarboxaldehyde $(0.107 \mathrm{~g}, 0.5 \mathrm{mmol})$ was added forming a bright red solution. Bis(tert-butyl)imidazol-2-ylidene ( $\left.\mathrm{I}^{t} \mathrm{Bu}\right)(0.09 \mathrm{~g}, 0.5 \mathrm{mmol})$ was then added and the obtained orange suspension was stirred for $2 \mathrm{~h}$ at $0{ }^{\circ} \mathrm{C}$. Toluene was added $(3 \mathrm{~mL})$ and the mixture was gently heated until all of the visible solid had dissolved. Slow cooling of the resulting 
solution afforded a crop of orange crystals $(0.173 \mathrm{~g}, 48 \%)$. Anal. Calcd for $\mathrm{C}_{34} \mathrm{H}_{63} \mathrm{FeGaN}_{2} \mathrm{O}_{2} \mathrm{Si}_{3}$ : C, 56.27; H, 8.75; N, 3.86. Found: C, 53.75; H, 8.42; N, 3.99. Sample consistently shows low value of the carbon content.

${ }^{1} \mathbf{H}$ NMR $\left(298 \mathrm{~K}, \boldsymbol{d}_{\mathbf{8}}\right.$-THF) $\delta(\mathrm{ppm})-0.86\left(6 \mathrm{H}\right.$, mult, $\left.\mathrm{CH}_{2} \mathrm{SiMe}_{3}\right),-0.03\left(27 \mathrm{H}, \mathrm{s}, \mathrm{Si}\left(\mathrm{CH}_{3}\right)_{3}\right)$, $1.65\left(9 \mathrm{H}, \mathrm{s}, \mathrm{C}\left(\mathrm{CH}_{3}\right)_{3}\right), 1.69\left(9 \mathrm{H}, \mathrm{s}, \mathrm{C}\left(\mathrm{CH}_{3}\right)_{3}\right), 3.75(1 \mathrm{H}, \mathrm{s}, \mathrm{FeCp})_{2}, 3.89(1 \mathrm{H}, \mathrm{s}, \mathrm{FeCp} 2), 3.94$ $(1 \mathrm{H}, \mathrm{s}, \mathrm{FeCp} 2), 4.14\left(5 \mathrm{H}, \mathrm{s}, \mathrm{FeCp}_{2}\right), 4.42\left(1 \mathrm{H}, \mathrm{s}, \mathrm{FeCp}_{2}\right), 6.24(1 \mathrm{H}, \mathrm{s}, \mathrm{OCH}), 7.67(1 \mathrm{H}, \mathrm{s}$, imidazole backbone $\mathrm{CH}), 8.36(1 \mathrm{H}, \mathrm{s}, \mathrm{C} 2-H) .{ }^{13} \mathbf{C}\left\{{ }^{1} \mathbf{H}\right\}$ NMR $\left(298 \mathbf{K}, \boldsymbol{d}_{\mathbf{8}}\right.$-THF) $\delta(\mathrm{ppm}) 2.0$ $\left(\mathrm{CH}_{2} \mathrm{SiMe}_{3}\right), 3.6\left(\mathrm{Si}\left(\mathrm{CH}_{3}\right)_{3}\right), 29.7\left(\mathrm{C}\left(\mathrm{CH}_{3}\right)_{3}\right), 31.3\left(\mathrm{C}\left(\mathrm{CH}_{3}\right)_{3}\right), 60.0\left(C\left(\mathrm{CH}_{3}\right)_{3}\right), 63.4\left(\mathrm{C}_{\left.\left(\mathrm{CH}_{3}\right)_{3}\right)}\right)$, $66.7\left(\mathrm{FeCp}_{2}-\mathrm{C}\right), 67.4\left(\mathrm{FeCp}_{2}-\mathrm{C}\right), 67.5\left(\mathrm{FeCp}_{2}-\mathrm{C}\right), 69.4\left(\mathrm{FeCp}_{2}-\mathrm{C}\right), 69.5\left(\mathrm{FeCp}_{2}-\mathrm{C}\right), 70.3$ $\left(\mathrm{FeCp}_{2}-\mathrm{C}\right), 100.7(\mathrm{CHO}), 120.2$ (imidazole backbone $\left.\mathrm{CH}\right), 130.6(\mathrm{NCHN}) 148.0\left(C_{\text {imidazole }}{ }^{-}\right.$ $\mathrm{CHO})$.

\section{Synthesis of $\left[a I^{t} \mathrm{BuC}(\mathrm{Ph})\left(\mathrm{CF}_{3}\right) \mathrm{OGaR}_{3}\right](7)$}

A solution of $\mathrm{Ga}\left(\mathrm{CH}_{2} \mathrm{SiMe}_{3}\right)_{3}(0.165 \mathrm{~g}, 0.5 \mathrm{mmol}$ in $10 \mathrm{~mL}$ hexane $)$ was cooled on an ice bath. $\alpha, \alpha, \alpha$-trifluoroacetophenone $(70 \mu \mathrm{L}, 0.5 \mathrm{mmol})$ was then added, followed by the addition of bis(tert-butyl)imidazol-2-ylidene ( $\left.\mathrm{I}^{t} \mathrm{Bu}\right)(0.09 \mathrm{~g}, 0.5 \mathrm{mmol})$. Obtained white suspension was stirred for $2 \mathrm{~h}$ at $0{ }^{\circ} \mathrm{C}$. Toluene was added $(2 \mathrm{~mL})$ and the mixture was gently heated until all of the visible solid had dissolved. Slow cooling of the resulting solution afforded oil which upon cooling at $-33{ }^{\circ} \mathrm{C}$ afforded a crop of colourless crystals $(0.215 \mathrm{~g}$, 63\%). Anal. Calcd for $\mathrm{C}_{31} \mathrm{H}_{58} \mathrm{~F}_{3} \mathrm{~N}_{2} \mathrm{OSi}_{3} \mathrm{Ga}$ : C, 54.29; H, 8.52; N, 4.08. Found: C, 54.11; H, $7.97 ; \mathrm{N}, 4.56$.

${ }^{1}$ H NMR (298 K, $\boldsymbol{d}_{\mathbf{8}}$-THF) $\delta(\mathrm{ppm})-0.83\left(6 \mathrm{H}, \mathrm{s}, \mathrm{CH}_{2} \mathrm{SiMe}_{3}\right),-0.05\left(27 \mathrm{H}, \mathrm{s}, \mathrm{Si}\left(\mathrm{CH}_{3}\right)_{3}\right), 1.51$ $\left(9 \mathrm{H}, \mathrm{s}, \mathrm{C}\left(\mathrm{CH}_{3}\right)_{3}\right), 1.72\left(9 \mathrm{H}, \mathrm{s}, \mathrm{C}\left(\mathrm{CH}_{3}\right)_{3}\right), 7.21(3 \mathrm{H}$, mult, imidazole backbone $\mathrm{CH}+o-\mathrm{CH})$, 7.53 (3H, mult, $m-\mathrm{CH}+p$-CH), $8.67(1 \mathrm{H}, \mathrm{s}, \mathrm{C} 2-H) .{ }^{13} \mathbf{C}\left\{{ }^{1} \mathbf{H}\right\}$ NMR $\left(298 \mathbf{K}, \boldsymbol{d}_{\mathbf{8}}\right.$-THF) $\delta(\mathrm{ppm})$ $\left.3.2\left(\mathrm{CH}_{2} \mathrm{SiMe}_{3}\right), 3.5\left(\mathrm{Si}\left(\mathrm{CH}_{3}\right)_{3}\right), 29.4\left(\mathrm{C} \mathrm{CH}_{3}\right)_{3}\right), 32.1\left(\mathrm{C}\left(\mathrm{CH}_{3}\right)_{3}\right), 60.6\left(\mathrm{C}_{\left.\left(\mathrm{CH}_{3}\right)_{3}\right), 66.6}\right.$ $\left(C\left(\mathrm{CH}_{3}\right)_{3}\right), 81.7$ (q, $\left.\mathrm{OC}(\mathrm{Ph}) \mathrm{CF}_{3}\right), 119.1$ (imidazole backbone $\left.\mathrm{CH}\right), 127.6(\mathrm{Ar}-\mathrm{CH}), 128.1$ (Ar$C \mathrm{H}), 129.4(\mathrm{Ar}-\mathrm{CH}), 134.1(\mathrm{NCHN}), 141.5\left(\mathrm{Ar}-\mathrm{C}_{i p s o}\right), 145.8\left(C_{\text {imidazole }}-\mathrm{OC}(\mathrm{Ph}) \mathrm{CF}_{3}\right) . \mathrm{CF}_{3}$ could not be confidentially assigned due to the low intensity of its resonances and noise in the baseline. ${ }^{19}$ F NMR (298 K, $\boldsymbol{d}_{\mathbf{8}}$-THF) $\delta$ (ppm) -69.92 br s (additional resonances arising from the decomposition of this sensitive substrate are observed in the ${ }^{19} \mathrm{~F}$ NMR spectrum of both product and starting material).

8. Synthesis of $\left[\left\{\mathrm{I}^{t} \mathrm{BuH}\right\}^{+}\left\{\left(\mathrm{p}-\mathrm{I}-\mathrm{C}_{6} \mathrm{H}_{4}\right) \mathrm{C}\left(\mathrm{CH}_{2}\right) \mathrm{OGaR}_{3}\right\}^{-}\right](\mathbf{8})$ 
To a cooled solution of $\mathrm{Ga}\left(\mathrm{CH}_{2} \mathrm{SiMe}_{3}\right)_{3}(0.165 \mathrm{~g}, 0.5 \mathrm{mmol}$ in $10 \mathrm{~mL}$ hexane $)$ 4'iodoacetophenone $(0.123 \mathrm{~g}, 0.5 \mathrm{mmol})$ was added followed by bis(tert-butyl)imidazol-2ylidene $\left(\mathrm{I}^{t} \mathrm{Bu}\right)(0.09 \mathrm{~g}, 0.5 \mathrm{mmol})$ and the obtained yellow suspension was stirred for $2 \mathrm{~h}$ at 0 ${ }^{\circ} \mathrm{C}$ and protected from light. Straw solid $(282 \mathrm{mg}, 74 \%)$ was isolated by filtration. Anal. Calcd for $\mathrm{C}_{31} \mathrm{H}_{60} \mathrm{GaIN}_{2} \mathrm{OSi}_{3}: \mathrm{C}, 49.14 ; \mathrm{H}, 7.98 ; \mathrm{N}, 3.70$. Found: C, 49.07; H, 7.81; N, 3.40.

${ }^{1} \mathbf{H}$ NMR $\left(298 \mathrm{~K}, \boldsymbol{d}_{\mathbf{8}}\right.$-THF) $\delta(\mathrm{ppm})-0.74\left(6 \mathrm{H}, \mathrm{s}, \mathrm{CH}_{2} \mathrm{SiMe}_{3}\right),-0.06\left(27 \mathrm{H}, \mathrm{s}, \mathrm{Si}\left(\mathrm{CH}_{3}\right)_{3}\right), 1.62$ $\left(18 \mathrm{H}, \mathrm{s}, \mathrm{C}\left(\mathrm{CH}_{3}\right)_{3}\right), 3.86\left(\mathrm{~s}, 1 \mathrm{H}, \mathrm{CH}_{2}\right), 4.12\left(\mathrm{~s}, 1 \mathrm{H}, \mathrm{CH}_{2}\right), 7.41(2 \mathrm{H}, \mathrm{d}, \mathrm{Ar}-\mathrm{CH}), 7.55(2 \mathrm{H}, \mathrm{d}$, Ar-CH), $7.78(2 \mathrm{H}$, s, imidazole backbone $\mathrm{CH}), 8.72(1 \mathrm{H}, \mathrm{s}, \mathrm{C} 2-H) .{ }^{13} \mathbf{C}\left\{{ }^{1} \mathbf{H}\right\}$ NMR (298 K, $\boldsymbol{d}_{\mathbf{8}}$-THF) $\delta(\mathrm{ppm}) 2.3\left(\mathrm{CH}_{2} \mathrm{SiMe}_{3}\right), 3.5\left(\mathrm{Si}\left(\mathrm{CH}_{3}\right)_{3}\right), 29.7\left(\mathrm{C}\left(\mathrm{CH}_{3}\right)_{3}\right), 61.2\left(\mathrm{C}_{\left.\left(\mathrm{CH}_{3}\right)_{3}\right), 79.9}\right.$ $\left(\mathrm{CH}_{2}=\mathrm{C}\right), 90.8(C-\mathrm{I}), 121.5$ (imidazole backbone $\left.\mathrm{CH}\right), 129.2(\mathrm{Ar}-\mathrm{CH}), 131.9(\mathrm{NCHN}), 136.3$ (Ar-CH), $145.8\left(\right.$ Ar- $\left.C_{i p s o}\right), 163.3(C-\mathrm{O})$.

9. Synthesis of $\left[\left\{\mathrm{I}^{t} \mathrm{BuH}\right\}^{+}\left\{\left(\mathrm{Me}_{3} \mathrm{C}_{6} \mathrm{H}_{2}\right) \mathrm{C}\left(\mathrm{CH}_{2}\right) \mathrm{OGaR}_{3}\right\}^{-}\right]$(9)

To a cooled solution of $\mathrm{Ga}\left(\mathrm{CH}_{2} \mathrm{SiMe}_{3}\right)_{3}(0.165 \mathrm{~g}, 0.5 \mathrm{mmol}$ in $10 \mathrm{~mL}$ hexane) 2',4',6'trimethylacetophenone $(0.081 \mathrm{~g}, 0.5 \mathrm{mmol})$ was added followed by bis(tert-butyl)imidazol-2ylidene $\left(\mathrm{I}^{t} \mathrm{Bu}\right)(0.09 \mathrm{~g}, 0.5 \mathrm{mmol})$ and the obtained yellow suspension was stirred for $2 \mathrm{~h}$ at 0 ${ }^{\circ} \mathrm{C}$. The off-white solid (203 mg, 60\%) was isolated by filtration. Anal. Calcd for $\mathrm{C}_{34} \mathrm{H}_{67} \mathrm{~N}_{2} \mathrm{OSi}{ }_{3} \mathrm{Ga}: \mathrm{C}, 60.60 ; \mathrm{H}, 10.02 ; \mathrm{N}, 4.16$. Found: $\mathrm{C}, 58.15 ; \mathrm{H}, 9.62 \mathrm{~N}, 3.88$. The sample shows consistently low value for carbon content. Small amounts of unreacted ketone are observed in NMR spectra.

${ }^{1} \mathbf{H}$ NMR $\left(298 \mathrm{~K}, \boldsymbol{d}_{\mathbf{8}}\right.$-THF) $\delta(\mathrm{ppm})-0.72\left(6 \mathrm{H}, \mathrm{s}, \mathrm{CH}_{2} \mathrm{SiMe}_{3}\right),-0.08\left(27 \mathrm{H}, \mathrm{s}, \mathrm{Si}\left(\mathrm{CH}_{3}\right)_{3}\right), 1.63$ $\left(18 \mathrm{H}, \mathrm{s}, \mathrm{C}\left(\mathrm{CH}_{3}\right)_{3}\right), 2.13\left(3 \mathrm{H}, \mathrm{s}, \mathrm{CH}_{3}\right), 2.33\left(6 \mathrm{H}, \mathrm{s}, \mathrm{CH}_{3}\right), 3.13\left(\mathrm{~s}, 1 \mathrm{H}, \mathrm{CH}_{2}\right), 3.85\left(\mathrm{~s}, 1 \mathrm{H}, \mathrm{CH}_{2}\right)$, $6.57(2 \mathrm{H}, \mathrm{s}, \mathrm{Ar}-\mathrm{CH}), 7.81(2 \mathrm{H}, \mathrm{s}$, imidazole backbone $\mathrm{CH}), 8.75(1 \mathrm{H}, \mathrm{s}, \mathrm{C} 2-H) .{ }^{13} \mathbf{C}\left\{{ }^{1} \mathbf{H}\right\}$ NMR $\left(298 \mathrm{~K}, \boldsymbol{d}_{\mathbf{8}}\right.$-THF) $\delta(\mathrm{ppm}) 2.3\left(\mathrm{CH}_{2} \mathrm{SiMe}_{3}\right), 3.5\left(\mathrm{Si}\left(\mathrm{CH}_{3}\right)_{3}\right), 21.00\left(\mathrm{Ar}-\mathrm{CH}_{3}\right), 21.3(\mathrm{Ar}-$

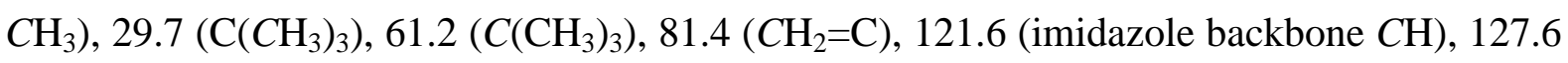
$(\mathrm{Ar}-\mathrm{CH}), 132.0(\mathrm{NCHN}), 133.3$ (Ar-C), 135.8 (Ar- $C), 145.1$ (Ar- $\left.C_{i p s o}\right), 165.7(C-\mathrm{O})$.

\section{Synthesis of $\left[\left\{\mathrm{SI}^{t} \mathrm{BuH}\right\}^{+}\left\{\left(\mathrm{p}-\mathrm{I}-\mathrm{C}_{6} \mathrm{H}_{4}\right) \mathrm{C}\left(\mathrm{CH}_{2}\right) \mathrm{OGaR}\right\}^{-}\right](\mathbf{1 0})$}

To a cooled solution of $\mathrm{Ga}\left(\mathrm{CH}_{2} \mathrm{SiMe}_{3}\right)_{3}(0.165 \mathrm{~g}, 0.5 \mathrm{mmol}$ in $10 \mathrm{~mL}$ hexane $)$ 4'iodoacetophenone $(0.123 \mathrm{~g}, 0.5 \mathrm{mmol})$ was added followed by bis(tert-butyl)imidazolin-2ylidene ( $\left.\mathrm{SI}^{t} \mathrm{Bu}\right)(0.09 \mathrm{~g}, 0.5 \mathrm{mmol})$ and the obtained of-white suspension was stirred for $2 \mathrm{~h}$ at $0{ }^{\circ} \mathrm{C}$ and protected from light. The peach-coloured solid (211 mg, 62\%) was isolated by 
filtration. Anal. Calcd for $\mathrm{C}_{31} \mathrm{H}_{62} \mathrm{~N}_{2} \mathrm{IOSi}_{3} \mathrm{Ga}: \mathrm{C}, 49.01 ; \mathrm{H}, 8.23 ; \mathrm{N}, 3.69$. Found: $\mathrm{C}, 48.26 ; \mathrm{H}$, $7.69 ; \mathrm{N}, 3.41$.

${ }^{1} \mathbf{H}$ NMR (298 K, d8-THF) $\delta(\mathrm{ppm})-0.74\left(6 \mathrm{H}, \mathrm{s}, \mathrm{CH}_{2} \mathrm{SiMe}_{3}\right),-0.05\left(27 \mathrm{H}, \mathrm{s}, \mathrm{Si}\left(\mathrm{CH}_{3}\right)_{3}\right), 1.37$ (18H, s, C(CH3) $\left.)_{3}\right), 3.85$ (s, 1H, $\left.\mathrm{CH}_{2}\right), 3.92$ (s, 4H, $\mathrm{CH}_{2}$ backbone), 4.13 (s, 1H, $\mathrm{CH}_{2}$ ), 7.43 $(2 \mathrm{H}, \mathrm{d}, \mathrm{Ar}-\mathrm{CH}), 7.56(2 \mathrm{H}, \mathrm{d}, \mathrm{Ar}-\mathrm{CH}), 7.88(1 \mathrm{H}, \mathrm{s}, \mathrm{C} 2-H) .{ }^{13} \mathbf{C}\left\{{ }^{1} \mathbf{H}\right\}$ NMR (298 K, d8-THF)

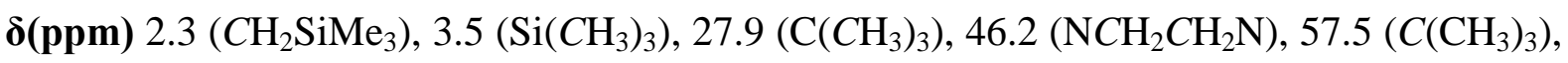
$79.8\left(\mathrm{CH}_{2}=\mathrm{C}\right), 90.9(C-\mathrm{I}), 129.1(\mathrm{Ar}-\mathrm{CH}), 136.4(\mathrm{Ar}-\mathrm{CH}), 145.8\left(\mathrm{Ar}-C_{i p s o}\right), 152.9(\mathrm{NCHN})$, $163.2(C-\mathrm{O})$.

\section{Synthesis of $\left[\left\{\mathrm{SI}^{t} \mathrm{BuH}\right\}^{+}\left\{\left(\mathrm{Me}_{3} \mathrm{C}_{6} \mathrm{H}_{2}\right) \mathrm{C}\left(\mathrm{CH}_{2}\right) \mathrm{OGaR}_{3}\right\}^{-}\right](\mathbf{1 1})$}

To a cooled solution of $\mathrm{Ga}\left(\mathrm{CH}_{2} \mathrm{SiMe}_{3}\right)_{3}(0.165 \mathrm{~g}, 0.5 \mathrm{mmol}$ in $10 \mathrm{~mL}$ hexane) 2',4',6'trimethylacetophenone $(0.081 \mathrm{~g}, 0.5 \mathrm{mmol})$ was added followed by bis(tert-butyl)imidazolin2-ylidene ( $\left.\mathrm{SI}^{t} \mathrm{Bu}\right)(0.09 \mathrm{~g}, 0.5 \mathrm{mmol})$ and the obtained white suspension was stirred for $2 \mathrm{~h}$ at $0{ }^{\circ} \mathrm{C}$ after which a white solid was isolated by filtration $(272 \mathrm{mg}, 80 \%)$. Anal. Calcd for $\mathrm{C}_{34} \mathrm{H}_{67} \mathrm{~N}_{2} \mathrm{OSi}_{3} \mathrm{Ga}: \mathrm{C}, 60.42 ; \mathrm{H}, 10.29 ; \mathrm{N}, 4.14$. Found: C, 60.66; H, 10.16; N, 4.65.

${ }^{1} \mathbf{H}$ NMR $\left(298 \mathrm{~K}, \mathbf{d}_{\mathbf{8}}\right.$-THF) $\delta(\mathrm{ppm})-0.72\left(6 \mathrm{H}, \mathrm{s}, \mathrm{CH}_{2} \mathrm{SiMe}_{3}\right),-0.06\left(27 \mathrm{H}, \mathrm{s}, \mathrm{Si}\left(\mathrm{CH}_{3}\right)_{3}\right), 1.37$ $\left(18 \mathrm{H}, \mathrm{s}, \mathrm{C}\left(\mathrm{CH}_{3}\right)_{3}\right), 2.15\left(3 \mathrm{H}, \mathrm{s}, \mathrm{CH}_{3}\right), 2.34\left(6 \mathrm{H}, \mathrm{s}, \mathrm{CH}_{3}\right), 3.16\left(\mathrm{~s}, 1 \mathrm{H}, \mathrm{CH}_{2}\right), 3.85\left(\mathrm{~s}, 4 \mathrm{H}, \mathrm{CH}_{2}\right.$ backbone), $3.86(\mathrm{~s}, 1 \mathrm{H}, \mathrm{CH}), 6.60(2 \mathrm{H}, \mathrm{s}, \mathrm{Ar}-\mathrm{CH}), 7.87(1 \mathrm{H}, \mathrm{s}, \mathrm{C} 2-H) .{ }^{13} \mathbf{C}\left\{{ }^{1} \mathbf{H}\right\}$ NMR (298 $\mathbf{K}, \mathbf{d}_{\mathbf{8}}$-THF) $\delta$ (ppm) $2.3\left(\mathrm{CH}_{2} \mathrm{SiMe}_{3}\right), 3.6\left(\mathrm{Si}\left(\mathrm{CH}_{3}\right)_{3}\right), 21.0\left(\mathrm{Ar}-\mathrm{CH}_{3}\right), 21.3\left(\mathrm{Ar}-\mathrm{CH}_{3}\right), 28.0$ $\left(\mathrm{C}\left(\mathrm{CH}_{3}\right)_{3}\right), 46.2\left(\mathrm{NCH}_{2} \mathrm{CH}_{2} \mathrm{~N}\right), 57.5\left(C\left(\mathrm{CH}_{3}\right)_{3}\right), 81.7\left(\mathrm{CH}_{2}=\mathrm{C}\right), 127.16(\mathrm{Ar}-\mathrm{CH}), 133.6$ (ArC), 135.9 (Ar-C), 145.1 (Ar- $\left.C_{i p s o}\right), 152.8(\mathrm{NCHN}), 165.6(C-\mathrm{O})$.

\section{Synthesis of $\left[\left\{\mathrm{I}^{t} \mathrm{BuH}\right\}^{+}\left\{\mathrm{Ph}_{2} \mathrm{C}=\mathrm{C}=\mathrm{NGaR}_{3}\right\}^{-}\right](\mathbf{1 2})$}

To a cooled solution of $\mathrm{Ga}_{\left(\mathrm{CH}_{2} \mathrm{SiMe}_{3}\right)_{3}}(0.165 \mathrm{~g}, 0.5 \mathrm{mmol}$ in $10 \mathrm{~mL}$ hexane $)$ diphenylacetonitrile (97 $\mathrm{mg}, 0.5 \mathrm{mmol}$ ) was added followed by bis(tert-butyl)imidazol-2ylidene $\left(\mathrm{I}^{\mathrm{t}} \mathrm{Bu}\right)(0.09 \mathrm{~g}, 0.5 \mathrm{mmol})$ and the obtained yellow suspension was stirred for $2 \mathrm{~h}$ at 0 ${ }^{\circ} \mathrm{C}$. Toluene was added $(3 \mathrm{~mL})$ and the mixture was gently heated until all of the visible solid had dissolved. Slow cooling of the resulting solution afforded yellow oil which upon standing provided X-ray quality crystals $(0.225 \mathrm{~g}, 64 \%)$. Anal. Calcd for $\mathrm{C}_{37} \mathrm{H}_{64} \mathrm{GaN}_{3} \mathrm{Si}_{3}: \mathrm{C}, 63.04 ; \mathrm{H}$, 9.15; N, 5.96. Found: C, 61.12; H, 8.51; N, 6.04. The sample consistently shows low values for carbon content. Small amounts of unreacted diphenylacetonitrile are observed in NMR spectra. 
${ }^{1} \mathbf{H}$ NMR $\left(298 \mathrm{~K}, \boldsymbol{d}_{\mathbf{8}}\right.$-THF) $\delta(\mathrm{ppm})-0.69\left(6 \mathrm{H}, \mathrm{s}, \mathrm{CH}_{2} \mathrm{SiMe}_{3}\right),-0.02\left(27 \mathrm{H}, \mathrm{s}, \mathrm{Si}\left(\mathrm{CH}_{3}\right)_{3}\right), 1.56$ $\left(18 \mathrm{H}, \mathrm{s}, \mathrm{C}\left(\mathrm{CH}_{3}\right)_{3}\right), 6.46(2 \mathrm{H}$, mult, $p-\mathrm{CH}), 6.92(4 \mathrm{H}, \mathrm{t}, m-\mathrm{CH}), 7.28(4 \mathrm{H}, \mathrm{t}, \mathrm{o}-\mathrm{CH}), 7.59(2 \mathrm{H}$, s, imidazole backbone $\mathrm{CH}), 8.68(1 \mathrm{H}, \mathrm{s}, \mathrm{C} 2-H) .{ }^{13} \mathbf{C}\left\{{ }^{1} \mathbf{H}\right\}$ NMR $\left(298 \mathbf{K}, \boldsymbol{d}_{\mathbf{8}}\right.$-THF $) \delta(\mathrm{ppm}) 2.7$ $\left(\mathrm{CH}_{2} \mathrm{SiMe}_{3}\right), 3.1\left(\mathrm{Si}\left(\mathrm{CH}_{3}\right)_{3}\right), 29.7\left(\mathrm{C}\left(\mathrm{CH}_{3}\right)_{3}\right), 55.7\left(\mathrm{Ph}_{2} \mathrm{C}=\mathrm{C}=\mathrm{N}\right), 61.1\left(C\left(\mathrm{CH}_{3}\right)_{3}\right), 117.9(\mathrm{Ar}-$ $C \mathrm{H}), 121.4$ (imidazole backbone $\mathrm{CH}), 123.1(\mathrm{Ar}-\mathrm{CH}), 128.4(\mathrm{Ar}-\mathrm{CH}), 131.9(\mathrm{NCHN}), 143.4$ $\left(\mathrm{Ph}_{2} \mathrm{C}=C=\mathrm{N}\right), 144.2\left(\mathrm{Ar}-C_{i p s o}\right)$.

\section{Synthesis of $\left[\left\{\mathrm{SI}^{t} \mathrm{BuH}\right\}^{+}\left\{\mathrm{Ph}_{2} \mathrm{C}=\mathrm{C}=\mathrm{NGaR}_{3}\right\}^{-}\right](\mathbf{1 3})$}

To a cooled solution of $\mathrm{Ga}\left(\mathrm{CH}_{2} \mathrm{SiMe}_{3}\right)_{3} \quad(0.165 \mathrm{~g}, 0.5 \mathrm{mmol}$ in $10 \mathrm{~mL}$ hexane $)$ diphenylacetonitrile (96 mg, $0.5 \mathrm{mmol}$ ) was added followed by bis(tert-butyl)imidazolin-2ylidene $\left(\mathrm{SI}^{t} \mathrm{Bu}\right)(0.09 \mathrm{~g}, 0.5 \mathrm{mmol})$ and the obtained yellow suspension was stirred for $2 \mathrm{~h}$ at 0 ${ }^{\circ} \mathrm{C}$. The yellow solid was isolated by filtration (305 $\mathrm{mg}, 86 \%$ ). Anal. Calcd for $\mathrm{C}_{37} \mathrm{H}_{66} \mathrm{~N}_{3} \mathrm{Si}_{3} \mathrm{Ga}: \mathrm{C}, 62.86 ; \mathrm{H}, 9.41 ; \mathrm{N}$, 5.94. Found: C, 62.15; H, 9.06; N, 6.28.

${ }^{1} \mathbf{H}$ NMR $\left(298 \mathrm{~K}, \mathbf{d}_{\mathbf{8}}\right.$-THF) $\delta(\mathrm{ppm})-0.68\left(6 \mathrm{H}, \mathrm{s}, \mathrm{CH}_{2} \mathrm{SiMe}_{3}\right), 0.00\left(27 \mathrm{H}, \mathrm{s}, \mathrm{Si}\left(\mathrm{CH}_{3}\right)_{3}\right), 1.28$ $\left(18 \mathrm{H}, \mathrm{s}, \mathrm{C}\left(\mathrm{CH}_{3}\right)_{3}\right), 3.75\left(\mathrm{~s}, 4 \mathrm{H}, \mathrm{CH}_{2}\right), 6.48(\mathrm{t}, 2 \mathrm{H}, p-\mathrm{CH}), 6.95(\mathrm{t}, 4 \mathrm{H}, m-\mathrm{CH}), 7.30(4 \mathrm{H}, \mathrm{d}, o-$ $\mathrm{CH}), 7.78(1 \mathrm{H}, \mathrm{s}, \mathrm{C} 2-H) .{ }^{13} \mathbf{C}\left\{{ }^{1} \mathbf{H}\right\}$ NMR (298 K, d8-THF) $\delta(\mathrm{ppm}) 2.8\left(\mathrm{CH}_{2} \mathrm{SiMe}_{3}\right), 3.1$ $\left(\mathrm{Si}\left(\mathrm{CH}_{3}\right)_{3}\right), 27.9\left(\mathrm{C}\left(\mathrm{CH}_{3}\right)_{3}\right), 46.0\left(\mathrm{NCH}_{2} \mathrm{CH}_{2} \mathrm{~N}\right), 55.6\left(\mathrm{Ph}_{2} \mathrm{C}=\mathrm{C}=\mathrm{N}\right), 57.5\left(C\left(\mathrm{CH}_{3}\right)_{3}\right), 118.0$ $(\mathrm{Ar}-\mathrm{CH}), \quad 123.0 \quad(\mathrm{Ar}-\mathrm{CH}), \quad 128.4 \quad(\mathrm{Ar}-\mathrm{CH}), \quad 143.1 \quad\left(\mathrm{Ph}_{2} \mathrm{C}=C=\mathrm{N}\right), 144.0 \quad\left(\mathrm{Ar}-C_{i p s o}\right), 152.6$ $(\mathrm{NCHN})$.

\section{Control reactions}

Several control reactions were conducted in sealed Young's tap NMR tubes to demonstrate that $\mathrm{GaR}_{3}$ interacts, but does not activate aldehydes (Fig S1) or ketones (Fig S2) and that $\mathrm{I}^{t} \mathrm{Bu}$ does not interact or activate aldehydes (Fig S3) or ketones (Fig S4) in any way. Furthermore, isolated crystalline sample of pre-formed $a \mathrm{I}^{t} \mathrm{Bu} \cdot \mathrm{GaR}_{3}$ has also been shown not to react with 4bromobenzaldehyde (Fig S5). Also, the mixture of equimolar amounts of $\mathrm{GaR}_{3}$ and $\mathrm{SI}^{t} \mathrm{Bu}$ (Fig S6) displays no signs of interaction between these two reagents even when heated over prolonged periods of time. Finally, ${ }^{1} \mathrm{H}$ and ${ }^{13} \mathrm{C}$ NMR spectra (Fig S7 and S8) of the crude reaction mixture of $\mathrm{I} t \mathrm{Bu} / \mathrm{GaR}_{3}$ pair with benzophenone reveals the deactivation of the pair and no reaction with this ketone. 

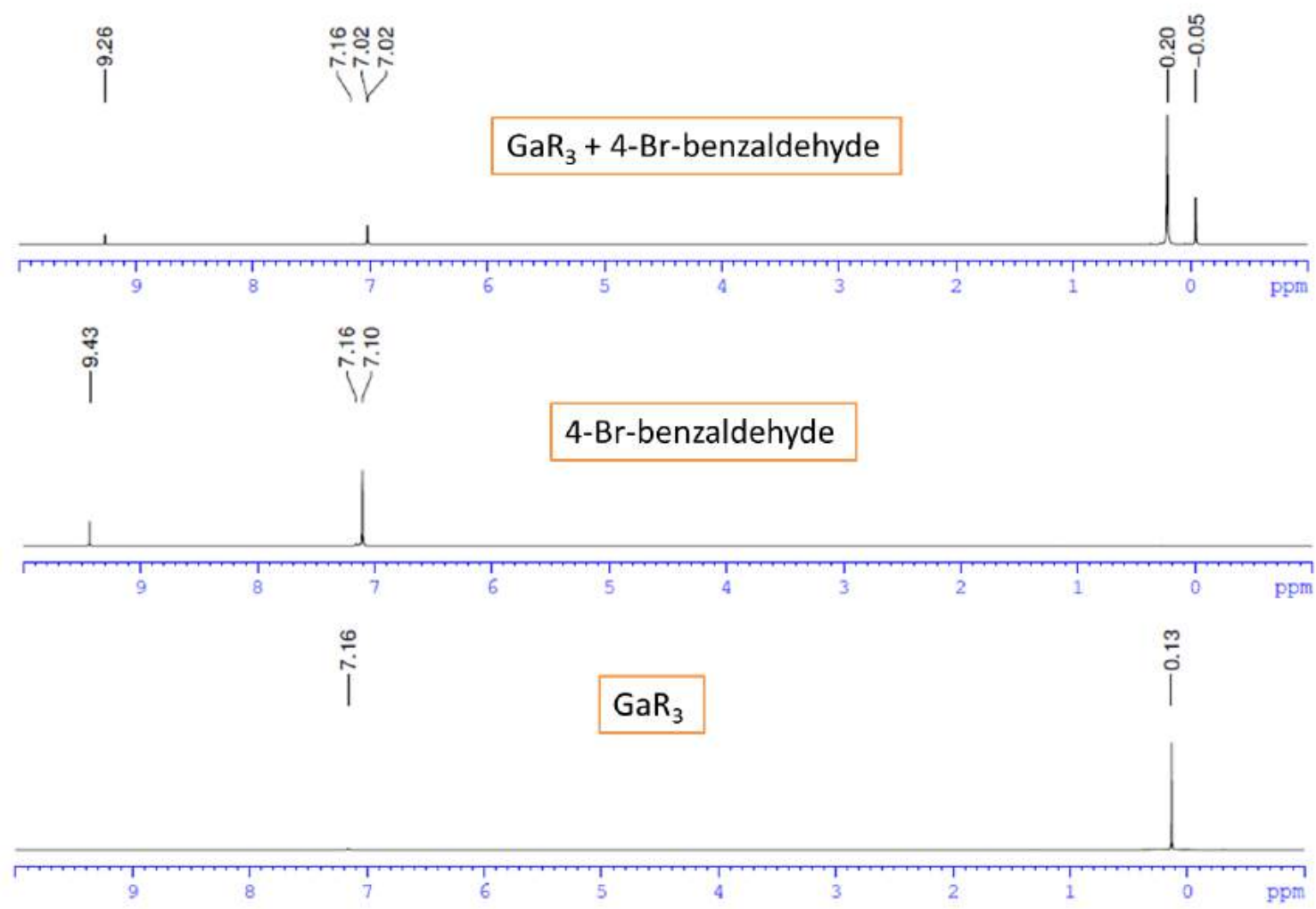

Figure S1: Comparison of ${ }^{1} \mathrm{H}$ NMR spectra in $\mathrm{C}_{6} \mathrm{D}_{6}$ of pure $\mathrm{GaR}_{3}$ (bottom), pure 4-bromobenzaldehyde (middle) and a mixture of equimolar amounts of $\mathrm{GaR}_{3}$ and 4-bromobenzaldehyde (top) revealing interaction between the two, but no reaction $\left[\mathrm{R}=\mathrm{CH}_{2} \mathrm{SiMe}_{3}\right]$.

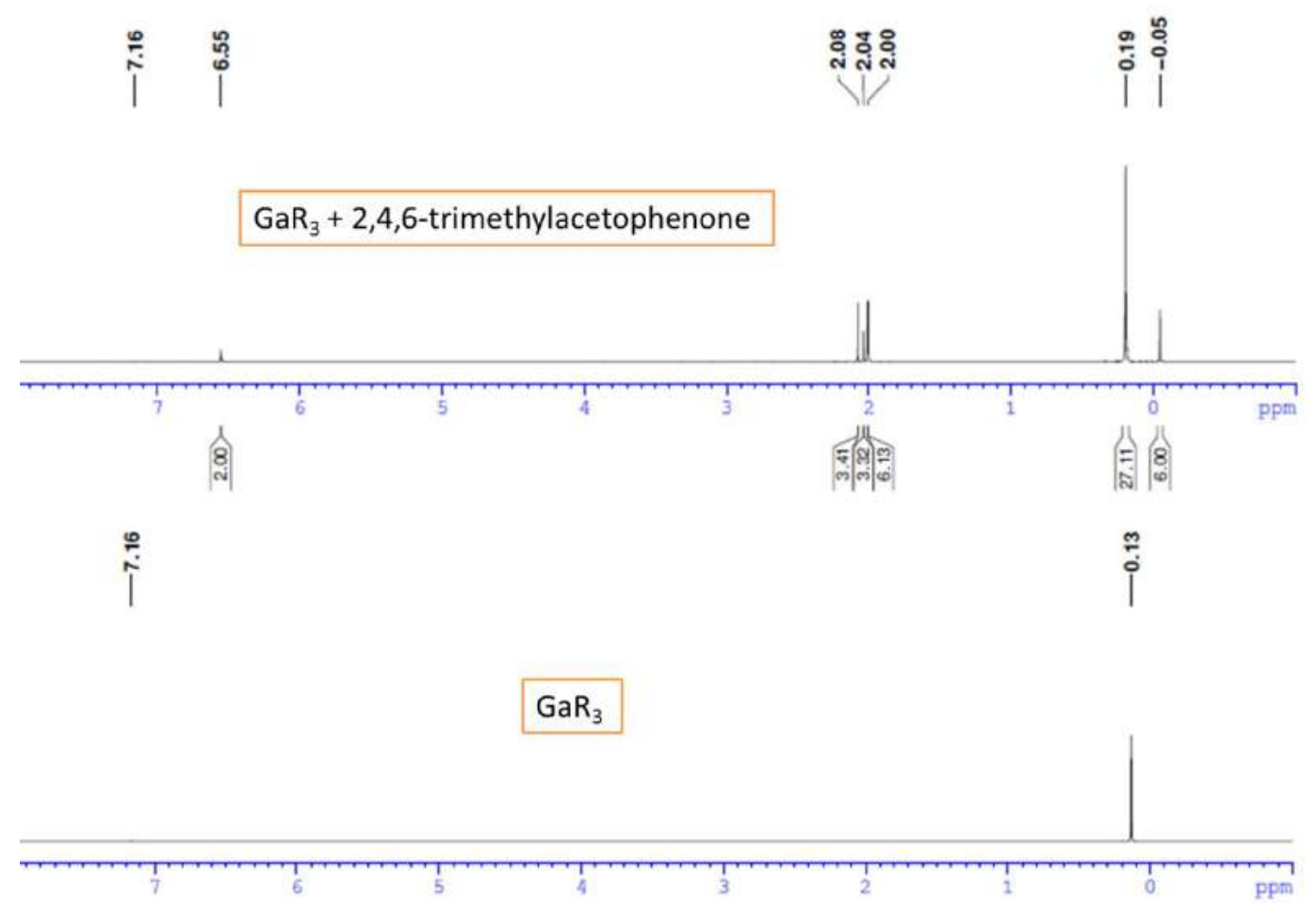

Figure S2: Comparison of ${ }^{1} \mathrm{H}$ NMR spectra in $\mathrm{C}_{6} \mathrm{D}_{6}$ of pure $\mathrm{GaR}_{3}$ (bottom) and a mixture of equimolar amounts of $\mathrm{GaR}_{3}$ and 2,4,6-trimethylacetophenone (top) revealing interaction between the two, but no reaction $\left[\mathrm{R}=\mathrm{CH}_{2} \mathrm{SiMe}_{3}\right]$. 

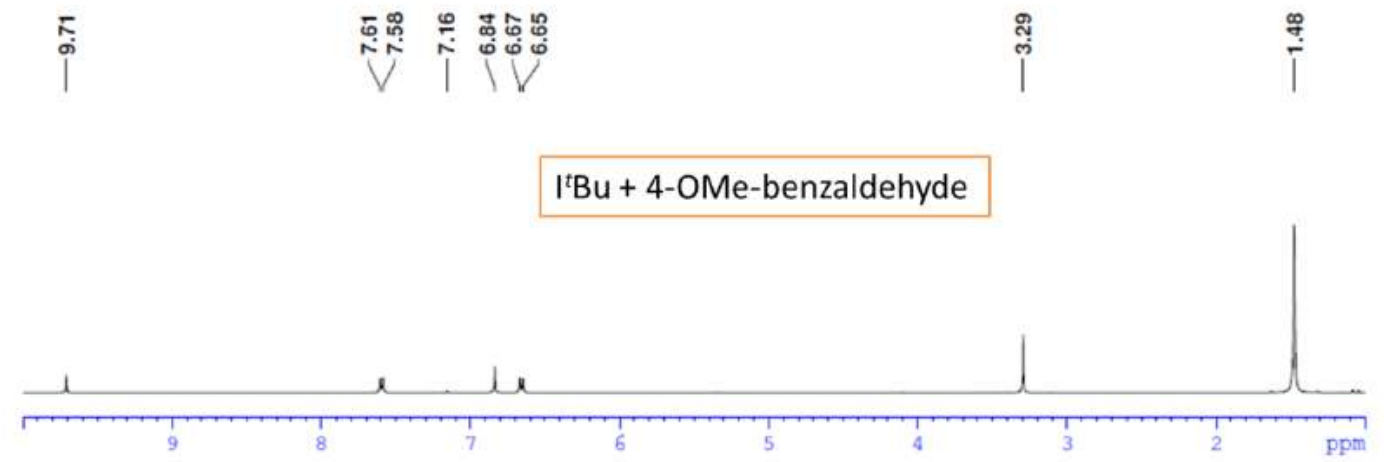

$\stackrel{\infty}{i}$

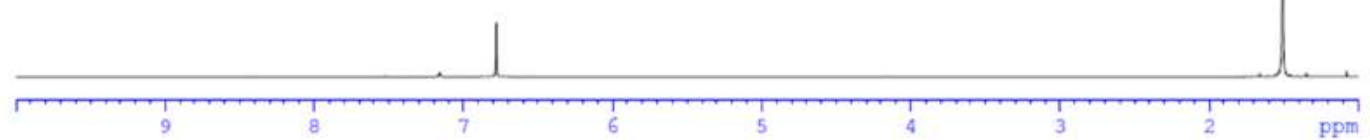

Figure S3: Comparison of ${ }^{1} \mathrm{H}$ NMR spectra in $\mathrm{C}_{6} \mathrm{D}_{6}$ of pure $\mathrm{I}^{t} \mathrm{Bu}$ (bottom) and a mixture of equimolar amounts of $\mathrm{I}^{t} \mathrm{Bu}$ and 4-anisaldehyde (top) revealing no interaction or any reaction between the two.
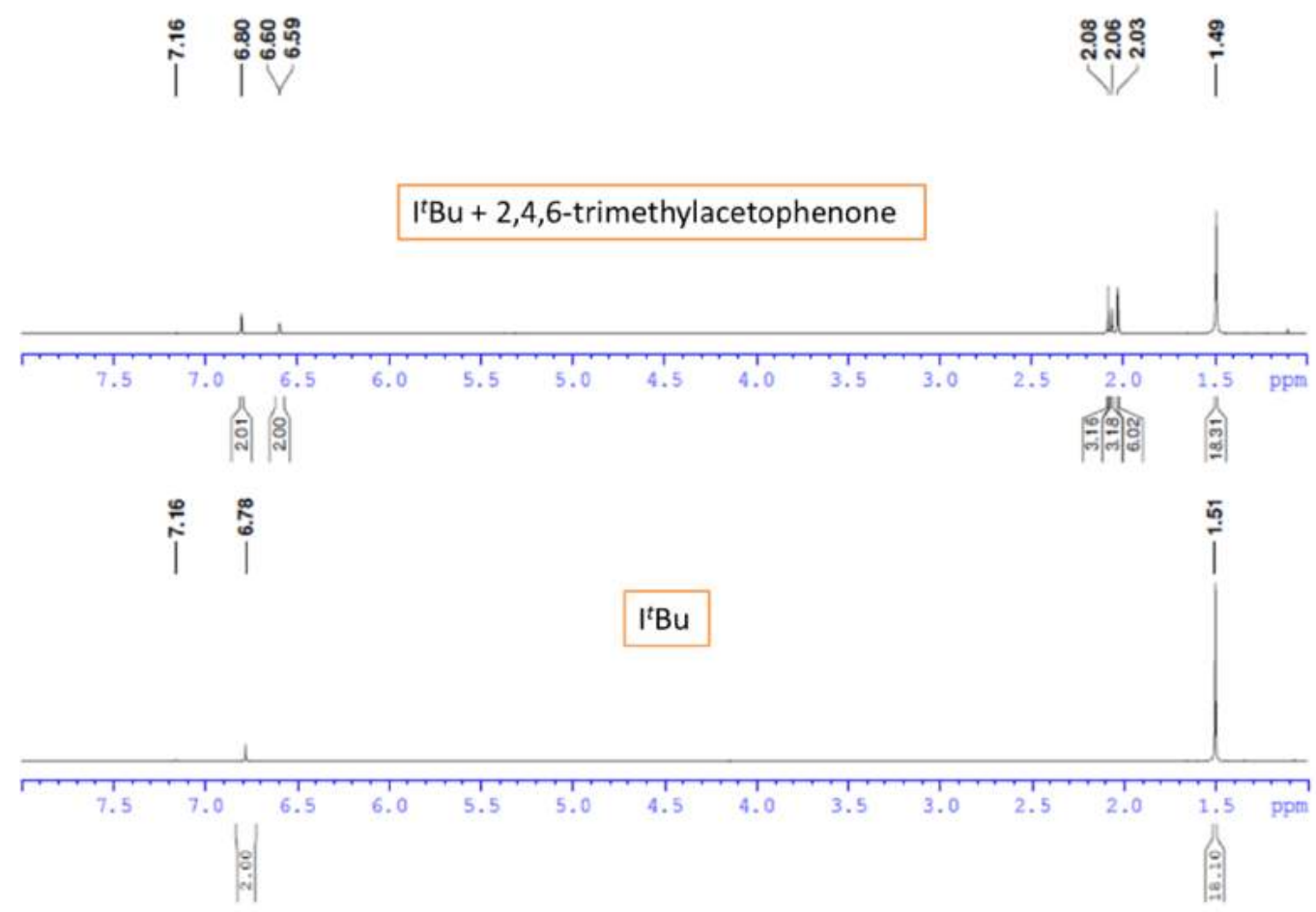

Figure S4: Comparison of ${ }^{1} \mathrm{H}$ NMR spectra in $\mathrm{C}_{6} \mathrm{D}_{6}$ of pure $\mathrm{I}^{t} \mathrm{Bu}$ (bottom) and a mixture of equimolar amounts of $\mathrm{I}^{t} \mathrm{Bu}$ and 2,4,6-trimethylacetophenone (top) revealing no interaction or any type of reaction between the two. 

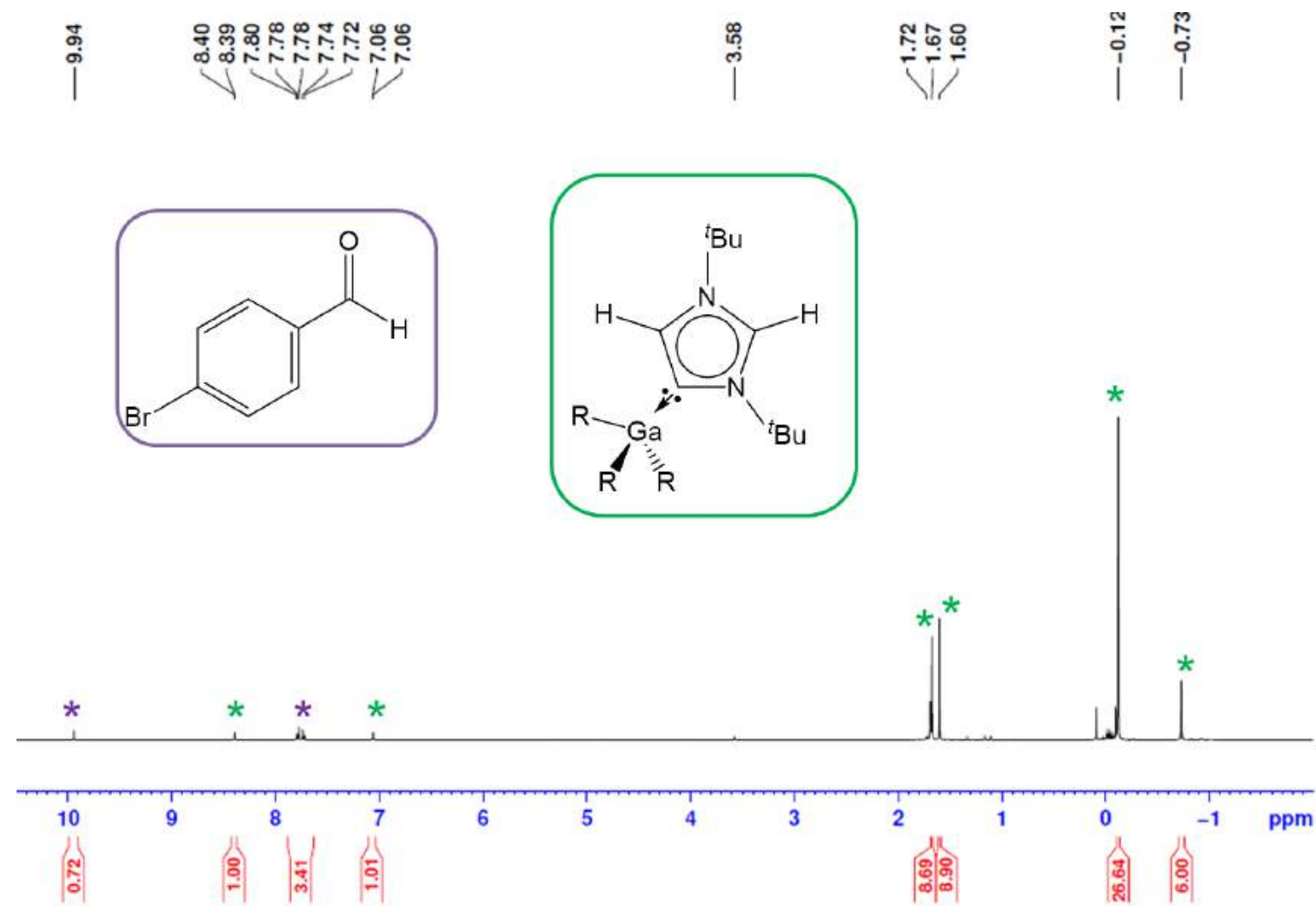

Figure S5: ${ }^{1} \mathrm{H}$ NMR spectrum of the mixture of $a \mathrm{I}^{t} \mathrm{Bu} \cdot \mathrm{GaR}_{3}$ and 4-bromobenzaldehyde in $\mathrm{d}_{8}$-THF standing for $48 \mathrm{~h}$ in a sealed Young's tap NMR tube at room temperature revealing no reaction between the two.

$\stackrel{\circ}{i}$
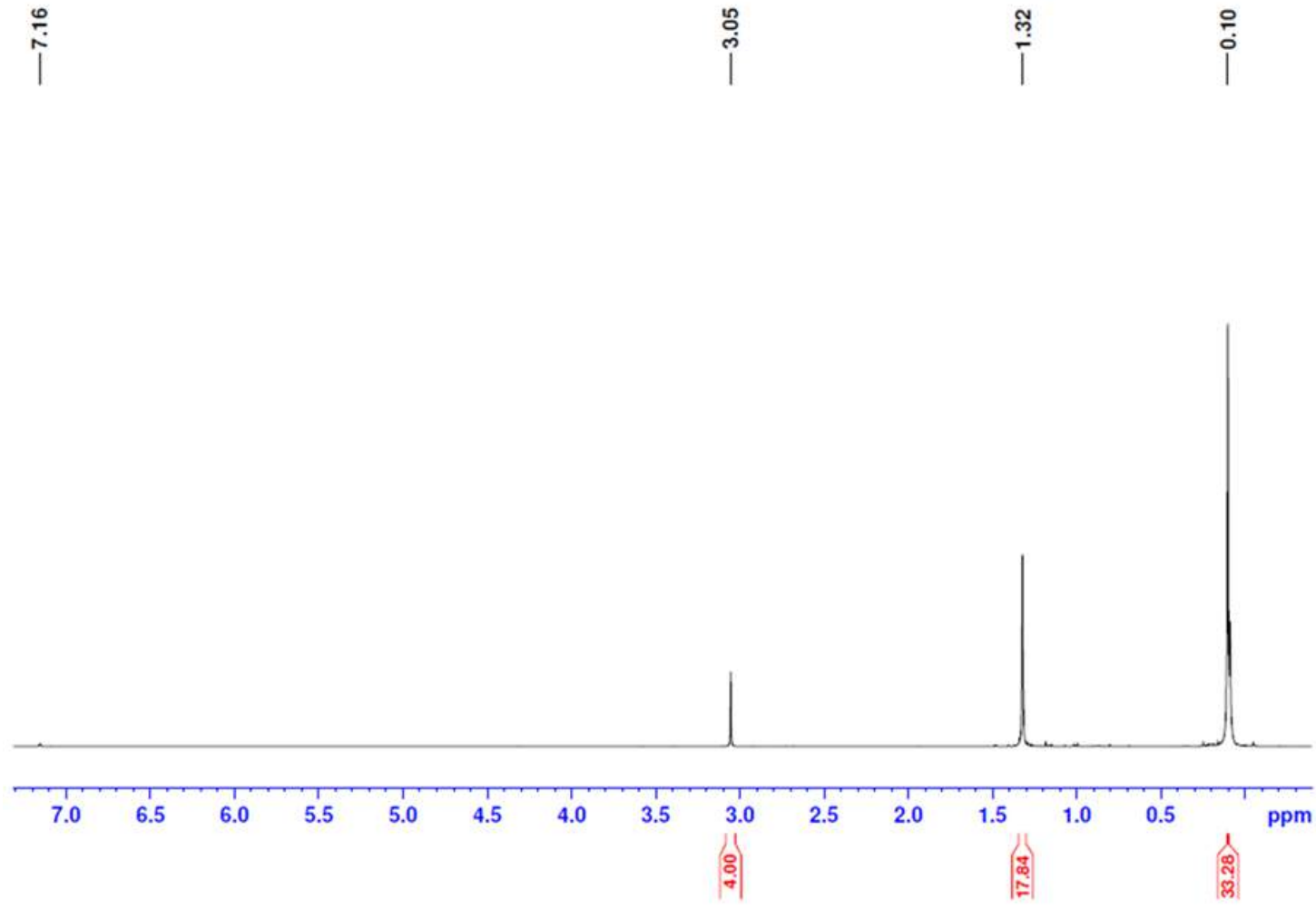

Figure S6: ${ }^{1} \mathrm{H}$ NMR spectrum of an equimolar mixture of $\mathrm{SI}^{t} \mathrm{Bu}$ and $\mathrm{GaR}_{3}$ after $9 \mathrm{~h}$ heating in $\mathrm{C}_{6} \mathrm{D}_{6}$ at $90{ }^{\circ} \mathrm{C}$ revealing no reaction between the two. 


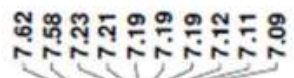

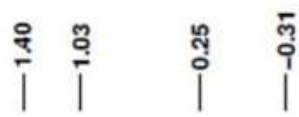

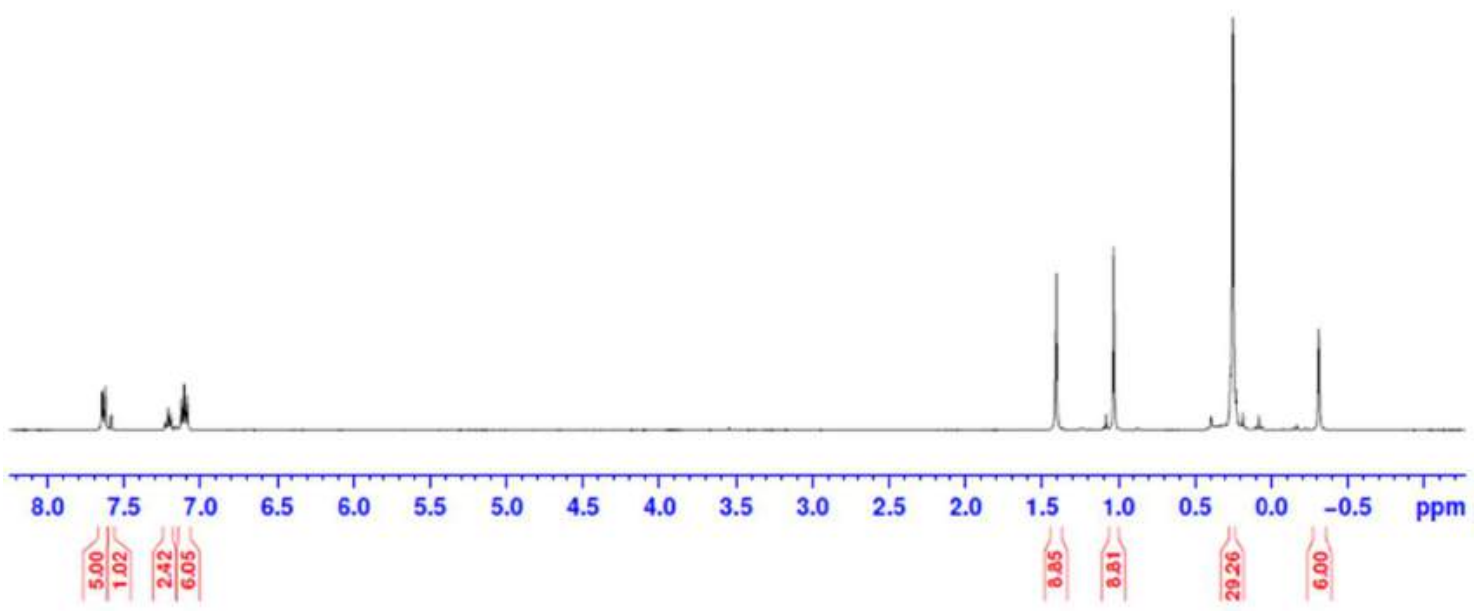

Figure S7: ${ }^{1} \mathrm{H}$ NMR spectrum of reaction mixture (benzophenone $+\mathrm{GaR}_{3}+\mathrm{I}^{t} \mathrm{Bu}$ in hexane at $0{ }^{\circ} \mathrm{C}$ for $2 \mathrm{~h}$ ) in $\mathrm{C}_{6} \mathrm{D}_{6}$ revealing the deactivation the FLP pair and no reactivity towards benzophenone.

5.<smiles>O=C(c1ccccc1)c1ccccc1</smiles><smiles>[R][Z9]([R])([R])[C@H]1C([2H])N(C(C)(C)C)C([2H])N1C(C)(C)C</smiles>

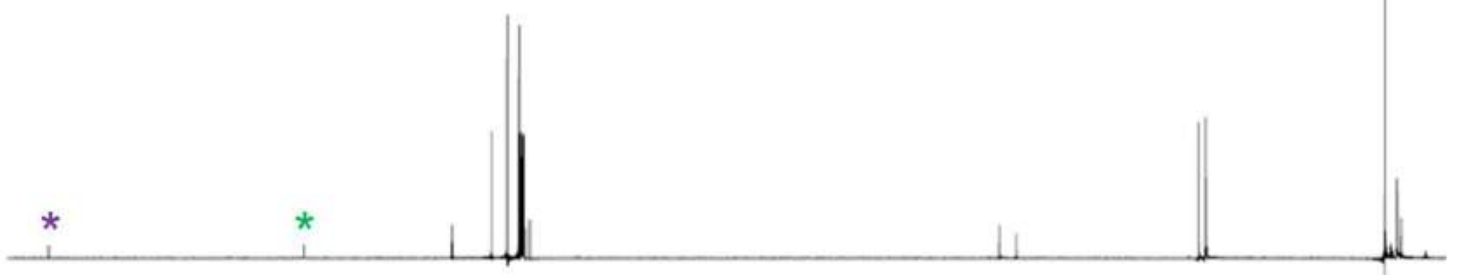

$\begin{array}{llllllllllllllllllll}190 & 180 & 170 & 160 & 150 & 140 & 130 & 120 & 110 & 100 & 90 & 80 & 70 & 60 & 50 & 40 & 30 & 20 & 10 & \mathrm{ppm}\end{array}$

Figure S8: ${ }^{13} \mathrm{CNMR}$ spectrum of reaction mixture (benzophenone $+\mathrm{GaR}_{3}+\mathrm{I}^{t} \mathrm{Bu}$ in hexane at $0{ }^{\circ} \mathrm{C}$ for $2 \mathrm{~h}$ ) in $\mathrm{C}_{6} \mathrm{D}_{6}$ revealing the deactivation the FLP pair and no reactivity towards benzophenone. 


\section{Variable Temperature ${ }^{1} \mathrm{H}$ NMR studies}

$0.08 \mathrm{~g}(0.25 \mathrm{mmol})$ of $\mathrm{GaR}_{3}$ was dissolved in $0.4 \mathrm{~mL}$ of $d_{8}$-THF in the glovebox, followed by addition of $40 \mathrm{mg}(0.25 \mathrm{mmol})$ of 4-bromobenzaldehyde. The reaction mixture was kept in a sealed Young's tap NMR tube and ${ }^{1} \mathrm{H}$ and ${ }^{13} \mathrm{C}$ NMR spectra were recorded at room temperature before the addition of $I^{t} \mathrm{Bu}$ (Fig S9 and S10). $45 \mathrm{mg}(0.25 \mathrm{mmol})$ of $\mathrm{I}^{t} \mathrm{Bu}$ was placed in the tube and as soon as all three components were mixed, the tube was placed into the NMR machine cooled to $230 \mathrm{~K}$. The reaction was regularly monitored by ${ }^{1} \mathrm{H}$ NMR spectroscopy and the temperature was gradually increased. At $230 \mathrm{~K}$, the presence of free starting materials and compound 2 is evident (Fig S11 and S12), as it is at $303 \mathrm{~K}$ (Fig S13). At $323 \mathrm{~K}$, species 3 is first observed (in addition to free components and compound 2) as can be seen in Fig S14. After leaving the tube to stand at $323 \mathrm{~K}$ for 15 min another ${ }^{1} \mathrm{H}$ NMR spectrum was recorded which revealed that species $\mathbf{2}$ ceased to exist and only compound $\mathbf{3}$ is present together with some starting material (Fig S15). This observation was further confirmed by the ${ }^{13} \mathrm{C}$ NMR spectrum (Fig S16). The overview of this experiment is shown in Fig S17.
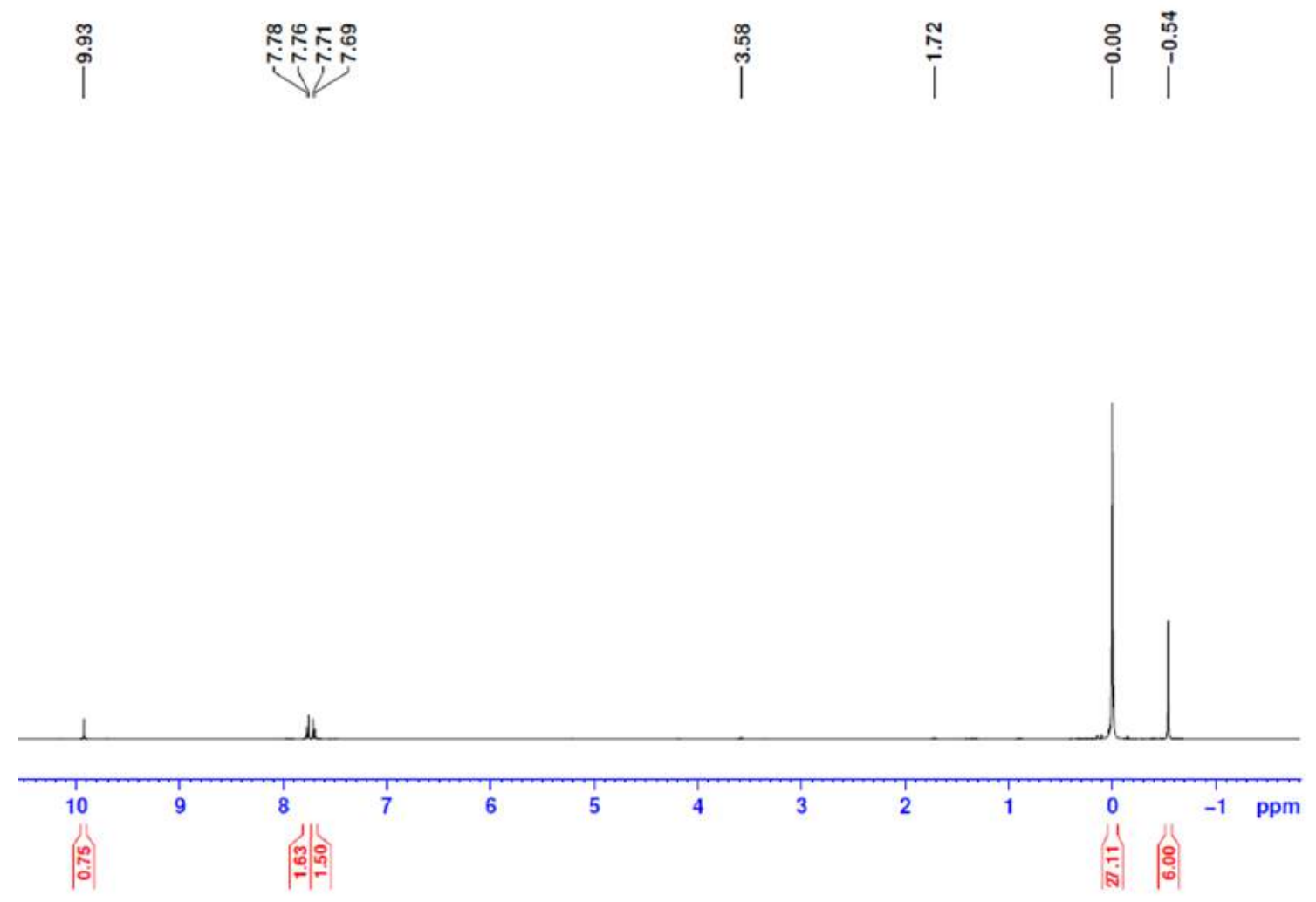

Figure S9: ${ }^{1} \mathrm{H}$ NMR spectrum of $\mathrm{GaR}_{3}$ and 4-bromobenzaldehyde in $\mathrm{d}_{8}$-THF at $298 \mathrm{~K}$. 

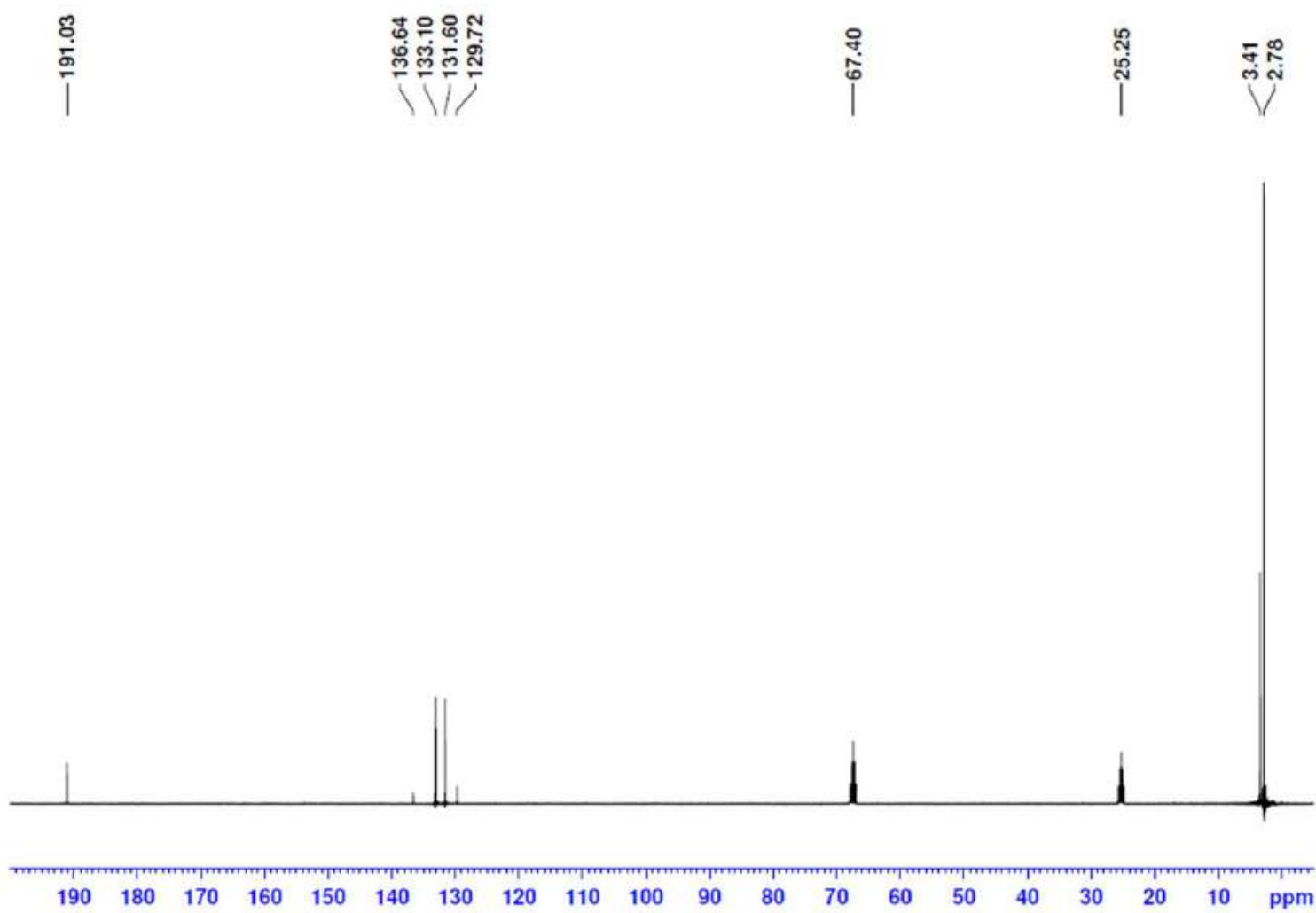

Figure S10: ${ }^{13} \mathrm{C}$ NMR spectrum of $\mathrm{GaR}_{3}$ and 4-bromobenzaldehyde in $\mathrm{d}_{8}$-THF at $298 \mathrm{~K}$.

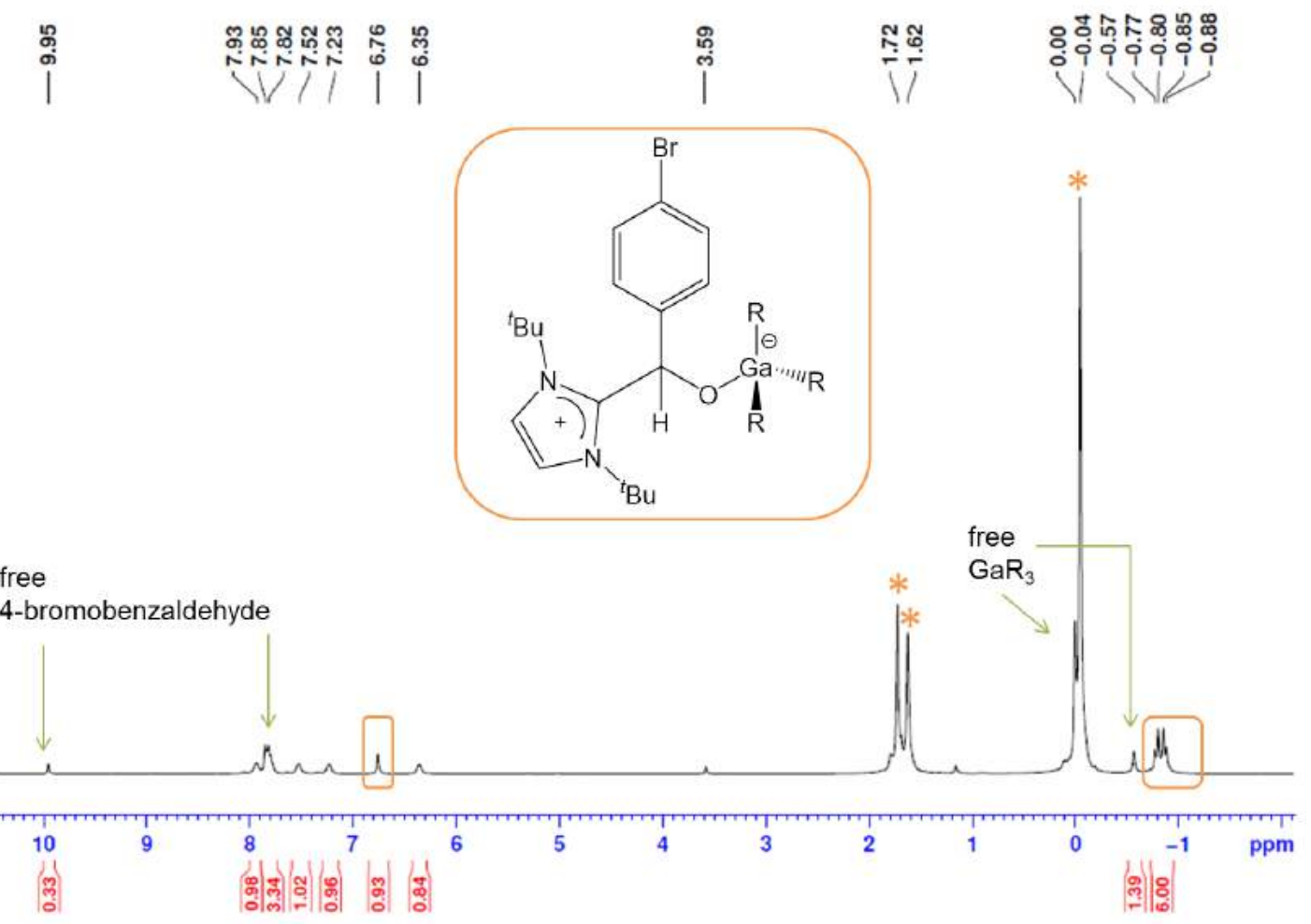

Figure S11: ${ }^{1} \mathrm{H}$ NMR spectrum of the reaction mixture recorded at $230 \mathrm{~K}$ as soon as $\mathrm{I}^{t} \mathrm{Bu}$ was added. 


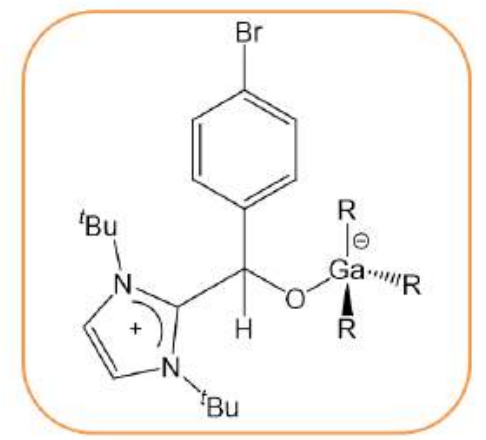

free

4-bromobenzaldehyde

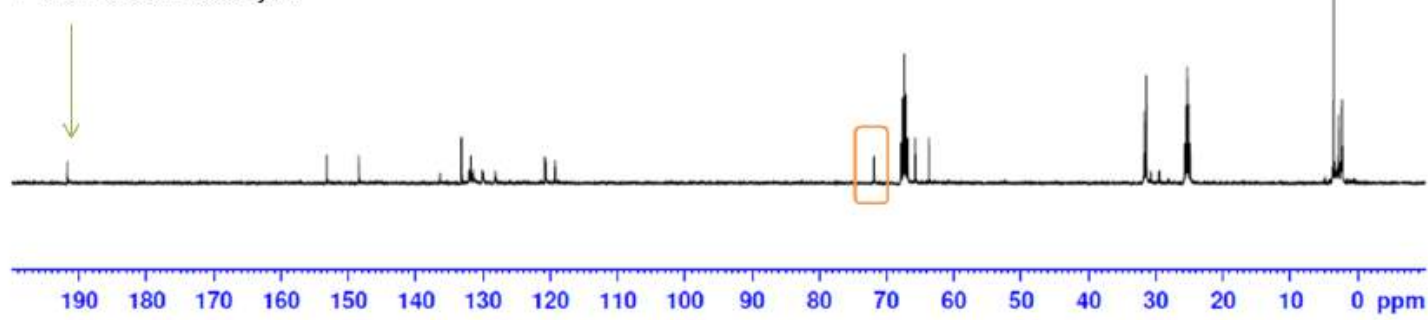

Figure S12: ${ }^{13} \mathrm{C}$ NMR spectrum of the reaction mixture recorded at $230 \mathrm{~K}$ as soon as $\mathrm{I}^{t} \mathrm{Bu}$ was added.
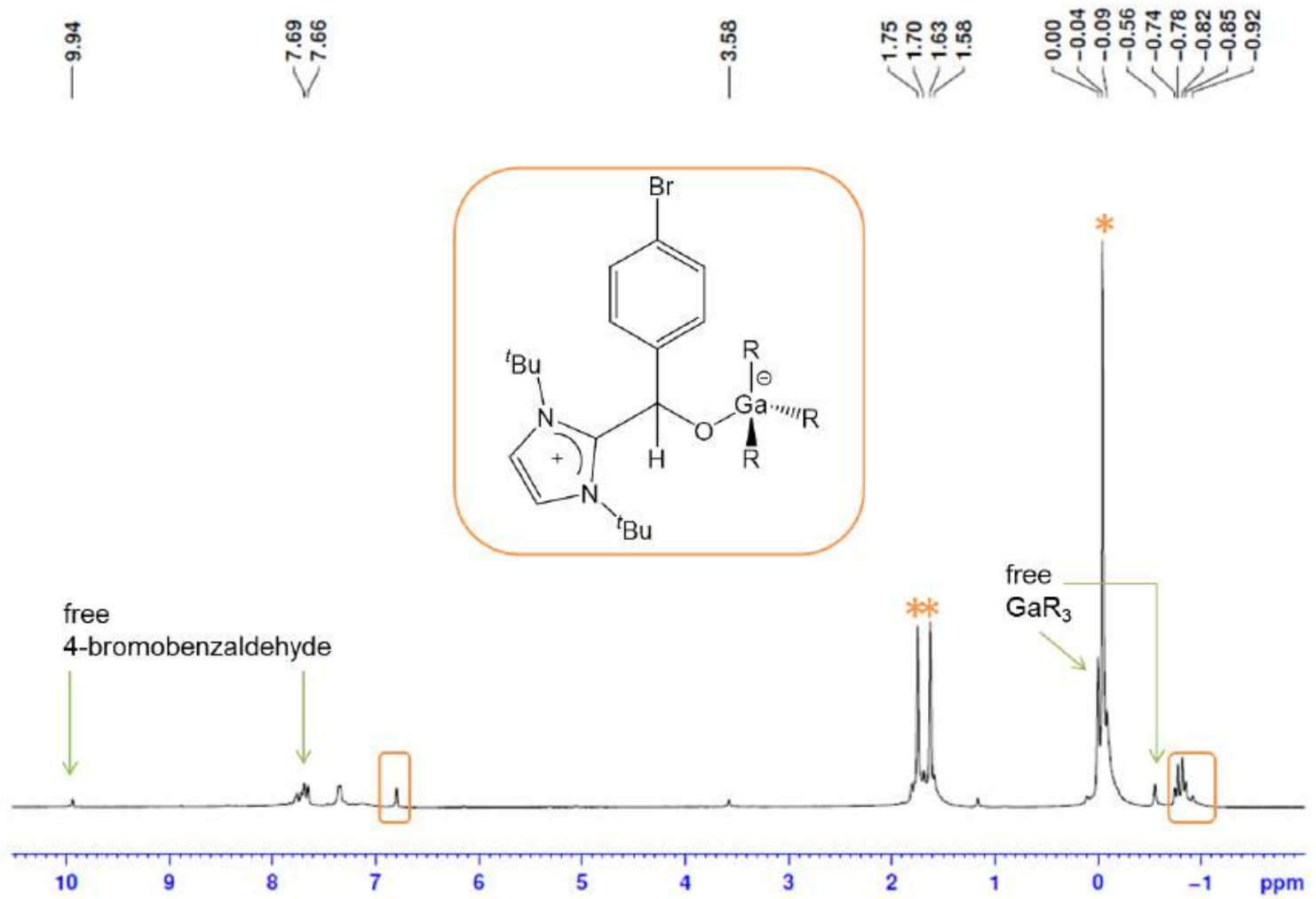

Figure S13: ${ }^{1} \mathrm{H}$ NMR spectrum of the reaction mixture recorded at $303 \mathrm{~K}$. 

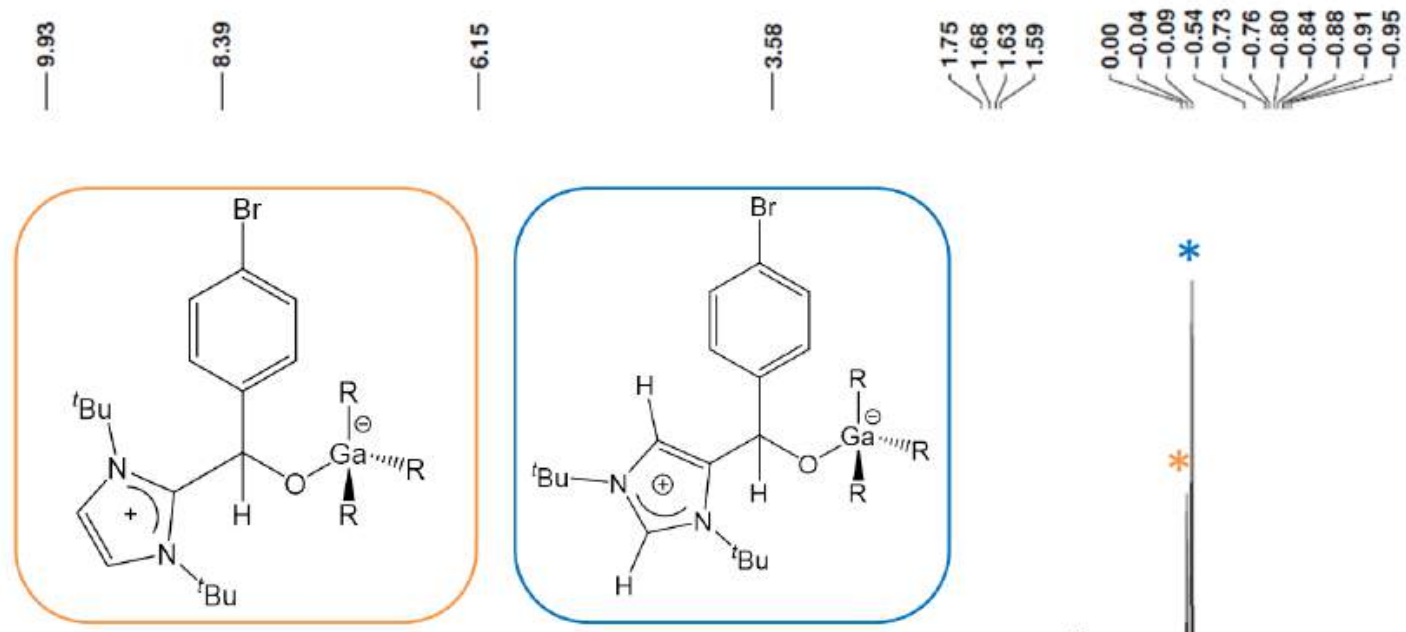

free

4-bromobenzaldehyde

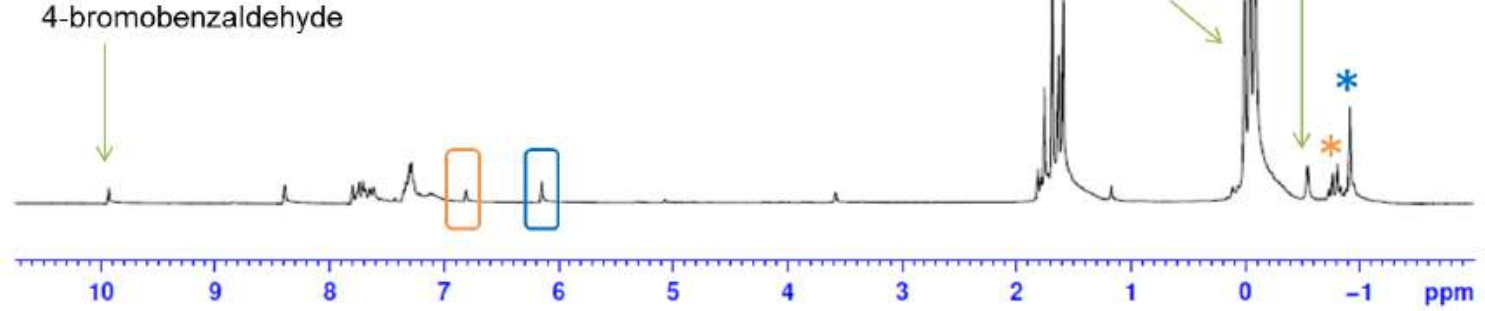

Figure S14: ${ }^{1} \mathrm{H}$ NMR spectrum of the reaction mixture recorded at $323 \mathrm{~K}$.
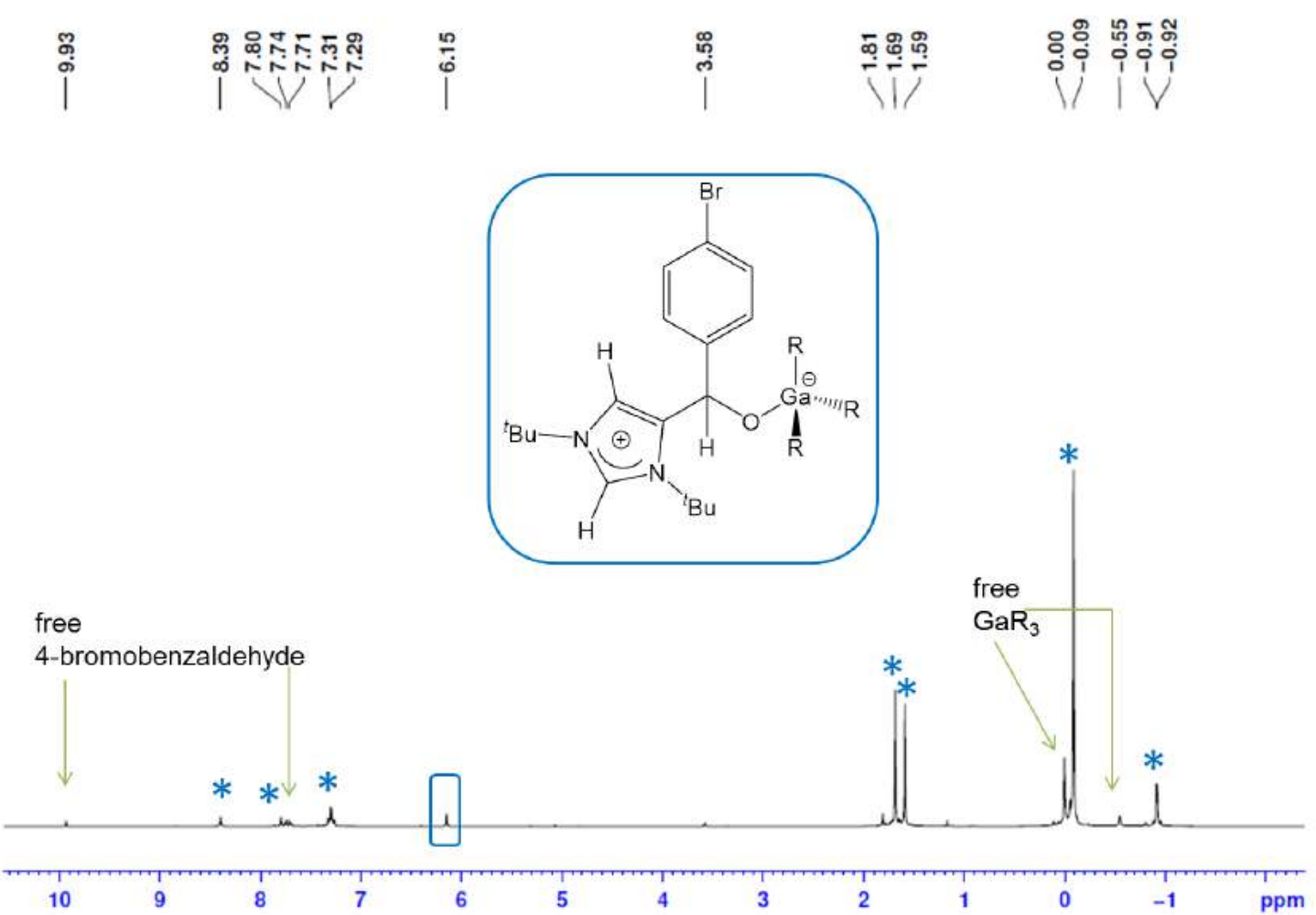

Figure S15: ${ }^{1} \mathrm{H}$ NMR spectrum of the reaction mixture recorded upon standing at $323 \mathrm{~K}$ for 15 min. 


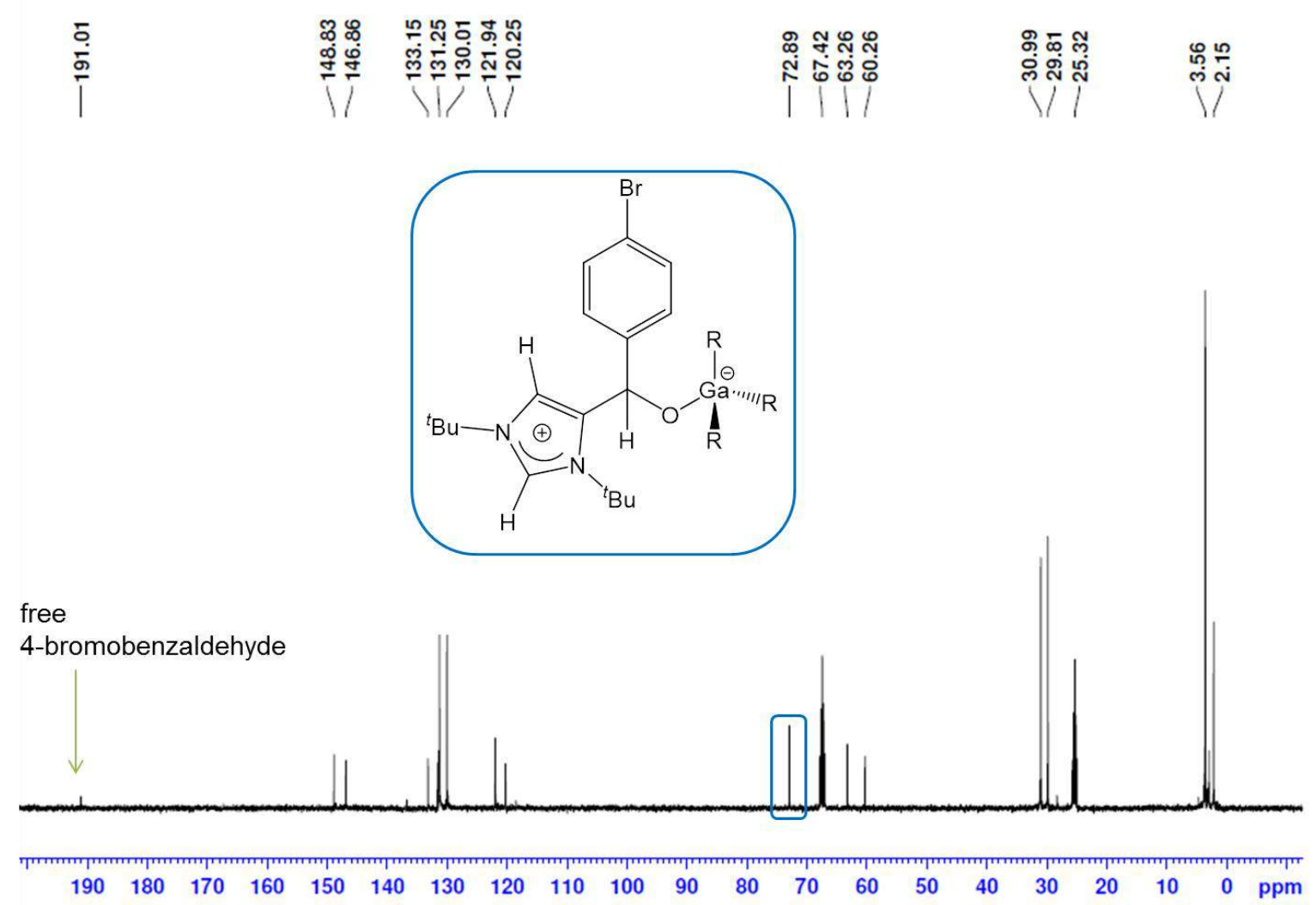

Figure S16: ${ }^{13} \mathrm{C}$ NMR spectrum of the reaction mixture recorded upon standing at $323 \mathrm{~K}$ for $15 \mathrm{~min}$.

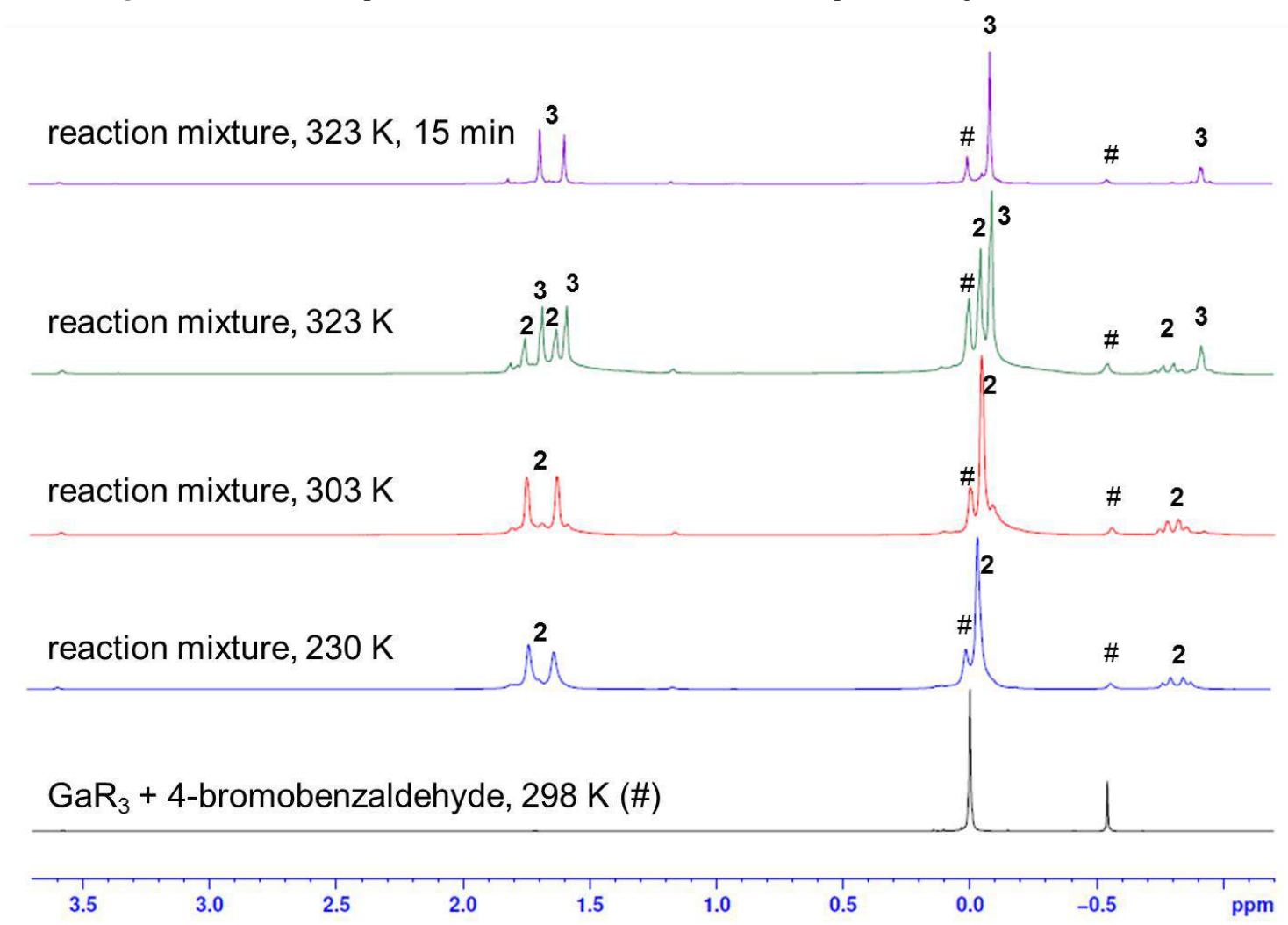

Figure S17 : ${ }^{1} \mathrm{H}$ NMR spectra (high-field region) of the reaction mixture in the $230-323 \mathrm{~K}$ temperature range. 


\section{DFT calculations}

Density Functional Theory (DFT) calculations ${ }^{5}$ were performed using the Gaussian computational package G03. ${ }^{6}$ In this series of calculations the geometries of the molecules and ions were optimised by employing the B3LYP density functionals ${ }^{7,8}$ and the $6-311 \mathrm{G}^{* *}$ basis set. ${ }^{9,10}$

Table S3: Comparison of calculated and experimentally found structural parameters for compounds 2 and 3.

\begin{tabular}{|c|c|c|c|c|}
\hline \multirow[b]{2}{*}{ Structural parameters } & \multicolumn{2}{|c|}{$\mathrm{I}^{t} \mathrm{BuCH}\left(\mathrm{C}_{6} \mathrm{H}_{4} \mathrm{Br}\right) \mathrm{OGaR}_{3}$ (2) } & \multicolumn{2}{|c|}{$a l^{t} \mathrm{BuCH}\left(\mathrm{C}_{6} \mathrm{H}_{4} \mathrm{Br}\right) \mathrm{OGaR}_{3}(3)$} \\
\hline & calculated & experimental & calculated & experimental \\
\hline $\mathrm{C}^{\mathrm{NHC}}-\mathrm{C}^{\text {substrate }}(\AA)$ & 1.547 & $1.525(3)$ & 1.532 & $1.541(10)$ \\
\hline Ga-O ${ }^{\text {substrate }}(\AA)$ & 2.016 & $1.9522(16)$ & 2.000 & $1.943(5)$ \\
\hline N-C-N $\left({ }^{\circ}\right)$ & 107.8 & $107.8(2)$ & 110.4 & $109.9(7)$ \\
\hline
\end{tabular}

Table S4: Comparison of calculated and experimentally found structural parameters for compounds 1 and 7.

\begin{tabular}{|c|c|c|c|c|}
\hline \multirow{3}{*}{$\begin{array}{c}\text { Structural } \\
\text { parameters } \\
\text { C }^{\mathrm{NHC}}-\mathrm{C}^{\text {substrate }}(\AA \AA)\end{array}$} & \multicolumn{2}{|c|}{$\mathrm{I}^{t} \mathrm{BuCH}_{2} \mathrm{OGaR}_{3}(\mathbf{1})$} & \multicolumn{2}{|c|}{$a I^{t} \mathrm{BuC}\left(\mathrm{CF}_{3}\right)\left(\mathrm{C}_{6} \mathrm{H}_{4}\right) \mathrm{OGaR}_{3}(7)$} \\
\hline & calculated & experimental & calculated & experimental \\
\hline & 1.519 & $1.505(3)$ & 1.552 & $1.544(3)$ \\
\hline Ga-O ${ }^{\text {substrate }}(\AA)$ & 2.010 & $1.9317(15)$ & 2.061 & $1.9692(14)$ \\
\hline $\mathbf{N}-\mathrm{C}-\mathrm{N}\left({ }^{\circ}\right)$ & 105.9 & $107.50(19)$ & 110.7 & $110.93(18)$ \\
\hline
\end{tabular}

Table S5: Comparison of total and relative energies for different variations of components of activation of 4 bromobenzaldehyde, formaldehyde and $\alpha, \alpha, \alpha$-trifluoroacetophenone.

\begin{tabular}{ccccc}
\hline & \multicolumn{2}{c}{ Total energies (a.u.) } & \multicolumn{2}{c}{ Relative energies $\left(\mathrm{kcal} \mathrm{mol}^{-1}\right.$ ) } \\
& normal & abnormal & normal & abnormal \\
$\mathrm{I}^{t} \mathrm{Bu}$ & -540.827324 & -540.799845 & 0.0 & 17.2 \\
$\mathrm{I}^{t} \mathrm{Bu} \cdot \mathrm{GaR}_{3}$ & -3811.699566 & -3811.710306 & 6.6 & 0.0 \\
\hline $\mathrm{I}^{t} \mathrm{Bu} \cdot \mathbf{p}-\mathrm{Br}-\mathrm{C}_{6} \mathrm{H}_{4} \cdot \mathrm{GaR}_{3}$ & -6730.925145 & -6730.931310 & 3.9 & 0.0 \\
$\mathbf{p}-\mathrm{Br}-\mathrm{C}_{6} \mathrm{H}_{4} \cdot \mathrm{GaR}_{3}$ & -6190.088449 & -6190.087370 & 0.0 & 0.7 \\
$\mathrm{I}^{t} \mathrm{Bu} \cdot \mathbf{p}-\mathrm{Br}-\mathrm{C}_{6} \mathrm{H}_{4}$ & -3460.012109 & -3460.012511 & 0.2 & 0.0 \\
\hline $\mathrm{I}^{t} \mathrm{Bu} \cdot \mathrm{CH}_{2} \mathbf{O} \cdot \mathrm{GaR}_{3}$ & -3926.286551 & -3926.286937 & 0.2 & 0.0 \\
\hline
\end{tabular}

\footnotetext{
${ }^{5}$ W. Kohn, A. D. Becke, R. G. Parr, J. Phys. Chem. 1996, 100, 12974.

${ }^{6}$ Gaussian 03, Revision B.0.5, M. J. Frisch, G. W. Trucks, H. B. Schlegel, G. E. Scuseria, M. A. Robb, J. R. Cheeseman, J. A. Montgomery, Jr., T. Vreven, K. N. Kudin, J. C. Burant, J. M. Millam, S. S. Iyengar, J. Tomasi, V. Barone, B. Mennucci, M. Cossi, G. Scalmani, N. Rega, G. A. Petersson, H. Nakatsuji, M. Hada, M. Ehara, K. Toyota, R. Fukuda, J. Hasegawa, M. Ishida, T. Nakajima, Y. Honda, O. Kitao, H. Nakai, M. Klene, X. Li, J. E. Knox, H. P. Hratchian, J. B. Cross, C. Adamo, J. Jaramillo, R. Gomperts, R. E. Stratmann, O. Yazyev, A. J. Austin, R. Cammi, C. Pomelli, J. W. Ochterski, P. Y. Ayala, K. Morokuma, G. A. Voth, P. Salvador, J. J. Dannenberg, V. G. Zakrzewski, S. Dapprich, A. D. Daniels, M. C. Strain, O. Farkas, D. K. Malick, A. D. Rabuck, K. Raghavachari, J. B. Foresman, J. V. Ortiz, Q. Cui, A. G. Baboul, S. Clifford, J. Cioslowski, B. B. Stefanov, G. Liu, A. Liashenko, P. Piskorz, I. Komaromi, R. L. Martin, D. J. Fox, T. Keith, M. A. AlLaham, C. Y. Peng, A. Nanayakkara, M. Challacombe, P. M. W. Gill, B. Johnson, W. Chen, M. W. Wong, C. Gonzalez, and J. A. Pople, Gaussian, Inc., Pittsburgh PA, 2003.

${ }^{7}$ A.D. Becke, Phys. Rev. A 1988, 38, 3098.

${ }^{8}$ C.T. Lee, W.T. Yang and R.G.Parr, Phys.Rev. B 1998, 37, 785.

${ }^{9}$ A. D. McLean and G. S. Chandler, J. Chem. Phys. 1980, 72, 5639.

${ }^{10}$ R. Krishnan, J. S. Binkley, R. Seeger and J. A. Pople, J. Chem. Phys. 1980, 72, 650.
} 


\begin{tabular}{ccccc}
\hline $\mathrm{I}^{t} \mathrm{Bu} \cdot \mathrm{C}(\mathrm{Ph}) \mathrm{CF}_{3} \mathrm{O} \cdot \mathrm{GaR}_{3}$ & -4494.503483 & -4494.517676 & 12.4 & 0.0 \\
$\mathrm{C}(\mathrm{Ph}) \mathrm{CF}_{3} \mathrm{O} \cdot \mathrm{GaR}_{3}$ & -3953.673983 & -3953.673983 & 0.0 & 0.0 \\
$\mathrm{I}^{t} \mathrm{Bu} \cdot \mathbf{C}(\mathbf{P h}) \mathrm{CF}_{3} \mathrm{O}$ & -1223.625316 & -1223.614338 & 0.0 & 6.9 \\
\hline
\end{tabular}

Table S6: Total energies of $\mathrm{GaR}_{3}$ and substrates used in this study.

\begin{tabular}{cc}
\hline & Total energies (a.u.) \\
$\mathbf{G a R}_{\mathbf{3}}$ & -3270.872410 \\
$\mathbf{p}-\mathbf{B r}_{\mathbf{6}} \mathbf{H}_{\mathbf{4}}$ & -2919.204596 \\
$\mathbf{C H}_{\mathbf{2}} \mathbf{O}$ & -114.536341 \\
$\mathbf{C F}_{\mathbf{3}} \mathbf{C}(\mathbf{P h}) \mathbf{O}$ & -682.794775 \\
\hline
\end{tabular}

Optimised geometry and energy of $\mathrm{Ga}\left(\mathrm{CH}_{2} \mathrm{SiMe}_{3}\right)_{3}, \mathrm{I}^{t} \mathrm{Bu}, a \mathrm{I}^{t} \mathrm{Bu}, \mathrm{I}^{t} \mathrm{Bu} \cdot \mathrm{GaR}_{3}$ and $a \mathrm{I}^{t} \mathrm{Bu} \cdot \mathrm{GaR}_{3}$ were obtained previously employing the same methods and basis set. ${ }^{11}$

1. Optimized geometry of 4-bromobenzaldehyde

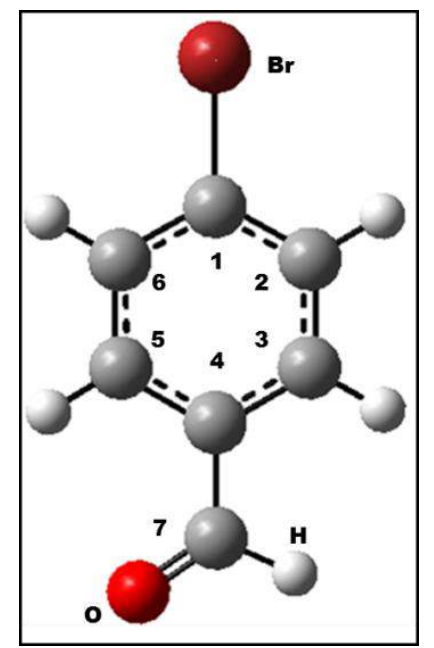

\begin{tabular}{cclrlr}
\hline \multicolumn{2}{c}{ Principal bond lengths $(\AA)$} & \multicolumn{2}{c}{ Principal bond angles $\left(^{\circ}\right)$} & \multicolumn{2}{c}{ Principal bond indices } \\
\hline $\mathbf{C}_{\mathbf{1}}-\mathbf{C}_{\mathbf{2}}$ & 1.393 & $\mathbf{C}_{\mathbf{1}}-\mathbf{C}_{\mathbf{2}}-\mathbf{C}_{\mathbf{3}}$ & 118.7 & $\mathbf{C}_{\mathbf{1}}-\mathbf{C}_{\mathbf{2}}$ & 1.41 \\
$\mathbf{C}_{\mathbf{2}}-\mathbf{C}_{\mathbf{3}}$ & 1.391 & $\mathbf{C}_{\mathbf{2}}-\mathbf{C}_{\mathbf{3}}-\mathbf{C}_{\mathbf{4}}$ & 120.6 & $\mathbf{C}_{\mathbf{2}}-\mathbf{C}_{\mathbf{3}}$ & 1.43 \\
$\mathbf{C}_{\mathbf{3}}-\mathbf{C}_{\mathbf{4}}$ & 1.397 & $\mathbf{C}_{\mathbf{3}}-\mathbf{C}_{\mathbf{4}}-\mathbf{C}_{\mathbf{5}}$ & 119.6 & $\mathbf{C}_{\mathbf{3}}-\mathbf{C}_{\mathbf{4}}$ & 1.38 \\
$\mathbf{C}_{\mathbf{4}}-\mathbf{C}_{\mathbf{5}}$ & 1.400 & $\mathbf{C}_{\mathbf{4}}-\mathbf{C}_{\mathbf{5}}-\mathbf{C}_{\mathbf{6}}$ & 120.4 & $\mathbf{C}_{\mathbf{4}}-\mathbf{C}_{\mathbf{5}}$ & 1.35 \\
$\mathbf{C}_{\mathbf{5}}-\mathbf{C}_{\mathbf{6}}$ & 1.387 & $\mathbf{C}_{\mathbf{5}}-\mathbf{C}_{\mathbf{6}}-\mathbf{C}_{\mathbf{1}}$ & 119.0 & $\mathbf{C}_{\mathbf{5}}-\mathbf{C}_{\mathbf{6}}$ & 1.46 \\
\hline
\end{tabular}

\footnotetext{
${ }^{11}$ M. Uzelac, A. Hernán-Gómez, D. R. Armstrong, A. R. Kennedy, E. Hevia, Chem. Sci., 2015, 6, 5719.
} 


\begin{tabular}{llllll}
\hline $\mathbf{C}_{6}-\mathbf{C}_{\mathbf{1}}$ & 1.396 & $\mathbf{C}_{\mathbf{6}}-\mathbf{C}_{\mathbf{1}}-\mathbf{C}_{\mathbf{2}}$ & 121.6 & $\mathbf{C}_{6}-\mathbf{C}_{1}$ & 1.39 \\
$\mathbf{C}_{\mathbf{1}}-\mathbf{B r}$ & 1.913 & $\mathbf{C}_{6}-\mathbf{C}_{\mathbf{1}}-\mathbf{B r}$ & 119.2 & $\mathbf{C}_{\mathbf{1}}-\mathbf{B r}$ & 1.05 \\
$\mathbf{C}_{\mathbf{4}}-\mathbf{C}_{7}$ & 1.481 & $\mathbf{C}_{\mathbf{3}}-\mathbf{C}_{\mathbf{4}}-\mathbf{C}_{7}$ & 120.1 & $\mathbf{C}_{4}-\mathbf{C}_{7}$ & 1.05 \\
$\mathbf{C}_{7}-\mathbf{O}$ & 1.209 & $\mathbf{C}_{4}-\mathbf{C}_{7}-\mathbf{H}$ & 114.5 & $\mathbf{C}_{7}-\mathbf{O}$ & 1.82 \\
$\mathbf{C}_{7}-\mathbf{H}$ & 1.112 & $\mathbf{C}_{4}-\mathbf{C}_{7}-\mathbf{O}$ & 124.6 & $\mathbf{C}_{7}-\mathbf{H}$ & 0.91 \\
\hline
\end{tabular}

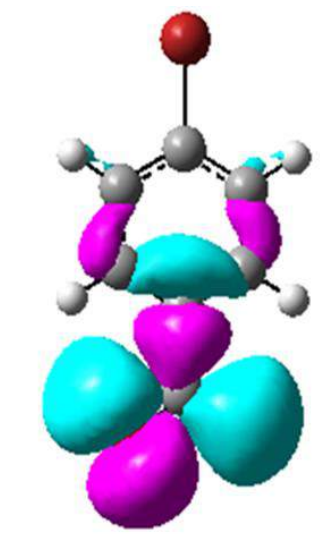

HOMO-1

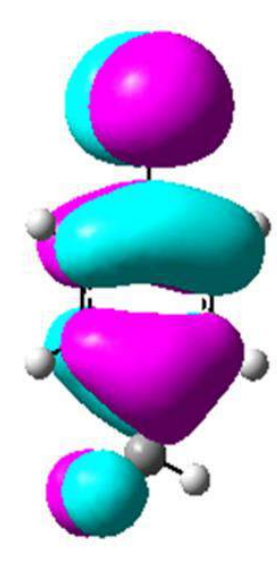

HOMO

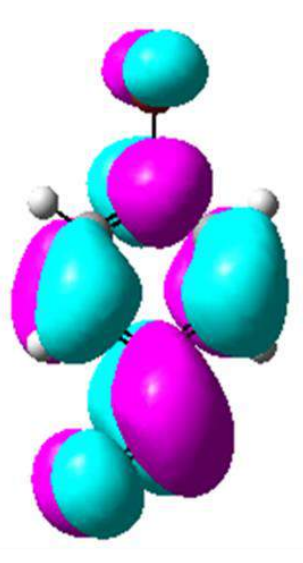

LUMO

Figure S18: Representation of molecular orbitals HOMO-1, HOMO and LUMO of p-bromobenzaldehyde.

2. Optimized geometry of $\alpha, \alpha, \alpha$-trifluoroacetophenone

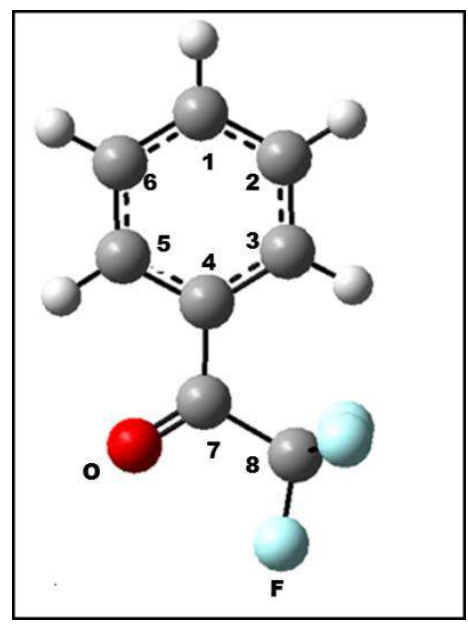

\begin{tabular}{cclclc}
\hline \multicolumn{2}{c}{ Principal bond lengths $(\AA)$} & \multicolumn{2}{c}{ Principal bond angles $\left(^{\circ}\right)$} & \multicolumn{2}{c}{ Principal bond indices } \\
\hline $\mathbf{C}_{\mathbf{1}}-\mathbf{C}_{\mathbf{2}}$ & 1.393 & $\mathbf{C}_{\mathbf{1}}-\mathbf{C}_{\mathbf{2}}-\mathbf{C}_{\mathbf{3}}$ & 120.2 & $\mathbf{C}_{\mathbf{1}}-\mathbf{C}_{\mathbf{2}}$ & 1.43 \\
$\mathbf{C}_{\mathbf{2}}-\mathbf{C}_{\mathbf{3}}$ & 1.391 & $\mathbf{C}_{\mathbf{2}}-\mathbf{C}_{\mathbf{3}}-\mathbf{C}_{\mathbf{4}}$ & 119.9 & $\mathbf{C}_{\mathbf{2}}-\mathbf{C}_{\mathbf{3}}$ & 1.44 \\
$\mathbf{C}_{\mathbf{3}}-\mathbf{C}_{\mathbf{4}}$ & 1.403 & $\mathbf{C}_{\mathbf{3}}-\mathbf{C}_{\mathbf{4}}-\mathbf{C}_{\mathbf{5}}$ & 119.4 & $\mathbf{C}_{\mathbf{3}}-\mathbf{C}_{\mathbf{4}}$ & 1.37 \\
$\mathbf{C}_{\mathbf{4}}-\mathbf{C}_{\mathbf{5}}$ & 1.404 & $\mathbf{C}_{\mathbf{4}}-\mathbf{C}_{\mathbf{5}}-\mathbf{C}_{\mathbf{6}}$ & 120.4 & $\mathbf{C}_{\mathbf{4}}-\mathbf{C}_{\mathbf{5}}$ & 1.35 \\
$\mathbf{C}_{\mathbf{5}}-\mathbf{C}_{\mathbf{6}}$ & 1.387 & $\mathbf{C}_{\mathbf{5}}-\mathbf{C}_{\mathbf{6}}-\mathbf{C}_{\mathbf{1}}$ & 119.9 & $\mathbf{C}_{\mathbf{5}}-\mathbf{C}_{\mathbf{6}}$ & 1.46 \\
$\mathbf{C}_{\mathbf{6}}-\mathbf{C}_{\mathbf{1}}$ & 1.396 & $\mathbf{C}_{\mathbf{6}}-\mathbf{C}_{\mathbf{1}}-\mathbf{C}_{\mathbf{2}}$ & 120.1 & $\mathbf{C}_{\mathbf{6}}-\mathbf{C}_{\mathbf{1}}$ & 1.42 \\
\hline
\end{tabular}




\begin{tabular}{lccccc}
\hline $\mathbf{C}_{7}-\mathbf{C}_{\mathbf{8}}$ & 1.558 & $\mathbf{C}_{\mathbf{4}}-\mathbf{C}_{\mathbf{7}}-\mathbf{C}_{\mathbf{8}}$ & 118.5 & $\mathbf{C}_{7}-\mathbf{C}_{\mathbf{8}}$ & 1.02 \\
$\mathbf{C}_{\mathbf{4}}-\mathbf{C}_{7}$ & 1.486 & $\mathbf{C}_{\mathbf{3}}-\mathbf{C}_{\mathbf{4}}-\mathbf{C}_{7}$ & 123.7 & $\mathbf{C}_{\mathbf{4}}-\mathbf{C}_{7}$ & 1.05 \\
$\mathbf{C}_{7}-\mathbf{O}$ & 1.207 & $\mathbf{C}_{\mathbf{4}}-\mathbf{C}_{7}-\mathbf{O}$ & 123.9 & $\mathbf{C}_{7}-\mathbf{O}$ & 1.80 \\
$\mathbf{C}_{8}-\mathbf{F}$ & $1.330 ; 1.352 ; 1.352$ & $\mathbf{C}_{7}-\mathbf{C}_{\mathbf{8}}-\mathbf{F}$ & $111.2 ; 110.9 ; 110.9$ & $\mathbf{C}_{\mathbf{8}}-\mathbf{F}$ & $0.91 ; 0.87 ; 0.87$ \\
\hline
\end{tabular}

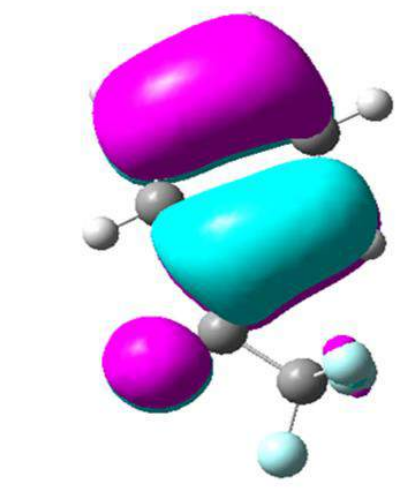

HOMO-1

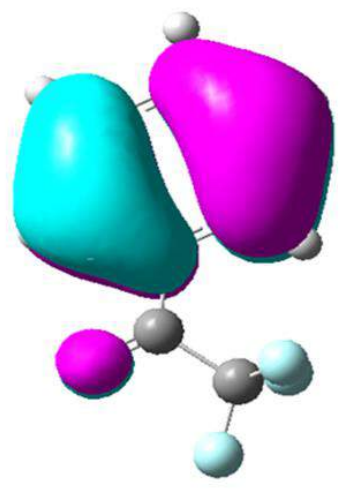

HOMO

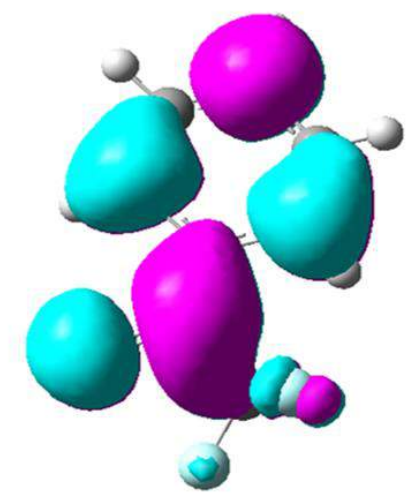

LUMO

Figure S19: Representation of molecular orbitals HOMO-1, HOMO and LUMO of trifluoroacetophenone.

3. Optimised geometry of formaldehyde

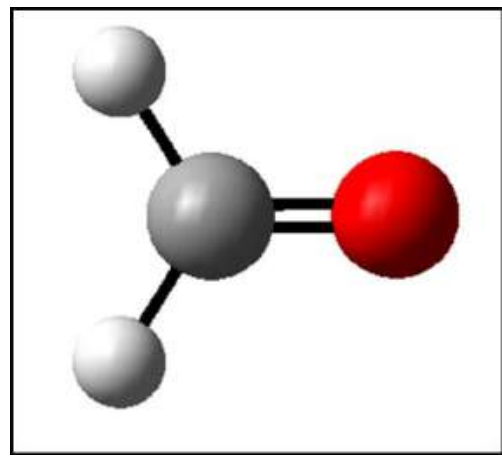

\begin{tabular}{lccc}
\hline \multicolumn{2}{c}{ Principal bond lengths $(\AA)$} & \multicolumn{2}{c}{ Principal bond angles $\left(^{\circ}\right)$} \\
\hline H-C & 1.110 & H-C-O & 122.3 \\
C-O & 1.200 & H-C-H & 115.5 \\
\hline
\end{tabular}

4. Optimised geometry of $\mathrm{GaR}_{3}+4$-bromobenzaldehyde 


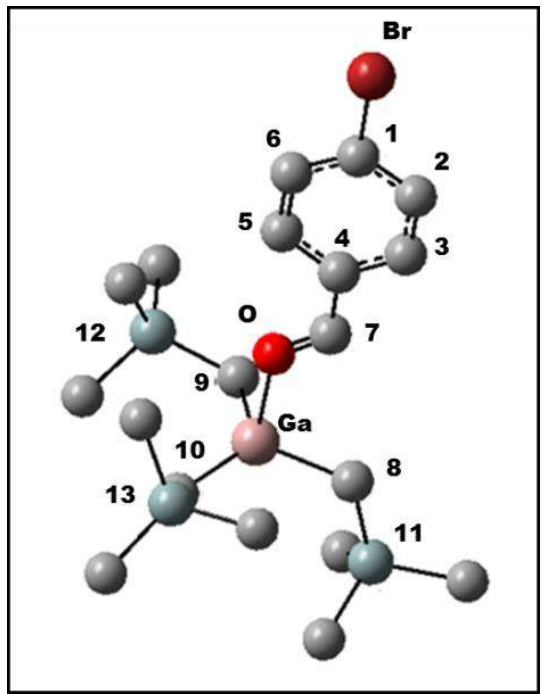

\begin{tabular}{|c|c|c|c|}
\hline \multicolumn{2}{|c|}{ Principal bond lengths $(\AA)$} & \multicolumn{2}{|c|}{ Principal bond angles $\left({ }^{\circ}\right)$} \\
\hline $\mathrm{C}_{1}-\mathrm{C}_{2}$ & 1.394 & $\mathrm{C}_{1}-\mathrm{C}_{2}-\mathrm{C}_{3}$ & 118.8 \\
\hline $\mathrm{C}_{2}-\mathrm{C}_{3}$ & 1.389 & $\mathrm{C}_{2}-\mathrm{C}_{3}-\mathrm{C}_{4}$ & 120.5 \\
\hline $\mathrm{C}_{3}-\mathrm{C}_{4}$ & 1.401 & $C_{3}-C_{4}-C_{5}$ & 119.7 \\
\hline $\mathrm{C}_{4}-\mathrm{C}_{5}$ & 1.404 & $\mathrm{C}_{4}-\mathrm{C}_{5}-\mathrm{C}_{6}$ & 120.2 \\
\hline $\mathrm{C}_{5}-\mathrm{C}_{6}$ & 1.385 & $C_{5}-C_{6}-C_{1}$ & 119.1 \\
\hline $\mathrm{C}_{6}-\mathrm{C}_{1}$ & 1.398 & $\mathrm{C}_{6}-\mathrm{C}_{1}-\mathrm{C}_{2}$ & 121.7 \\
\hline $\mathrm{C}_{1}-\mathrm{Br}$ & 1.907 & $\mathrm{C}_{6}-\mathrm{C}_{1}-\mathrm{Br}$ & 119.1 \\
\hline $\mathrm{C}_{4}-\mathrm{C}_{7}$ & 1.462 & $C_{3}-C_{4}-C_{7}$ & 119.3 \\
\hline $\mathrm{C}_{7}-\mathrm{H}$ & 1.102 & $\mathrm{C}_{4}-\mathrm{C}_{7}-\mathrm{H}$ & 116.4 \\
\hline $\mathrm{C}_{7}-\mathrm{O}$ & 1.225 & $\mathrm{C}_{4}-\mathrm{C}_{7}-\mathrm{O}$ & 123.8 \\
\hline Ga-O & 2.284 & $\mathrm{C}_{7}-\mathrm{O}-\mathbf{G a}$ & 126.6 \\
\hline $\mathrm{Ga}^{-\mathrm{C}_{8}}$ & 2.019 & $\mathrm{O}-\mathrm{Ga}-\mathrm{C}_{8}$ & 96.7 \\
\hline Ga-C ${ }_{9}$ & 2.015 & O-Ga-C ${ }_{9}$ & 94.1 \\
\hline $\mathrm{Ga}-\mathrm{C}_{10}$ & 2.009 & O-Ga-C $C_{10}$ & 101.4 \\
\hline $\mathrm{C}_{8}-\mathrm{Si}_{11}$ & 1.883 & $\mathrm{Ga}-\mathrm{C}_{8}-\mathrm{Si}_{11}$ & 118.2 \\
\hline $\mathrm{C}_{9}-\mathrm{Si}_{12}$ & 1.878 & $\mathrm{Ga}-\mathrm{C}_{9}-\mathrm{Si}_{12}$ & 122.0 \\
\hline $\begin{array}{l}\mathrm{C}_{10}-\mathrm{Si}_{13} \\
\mathrm{Si}_{11}-\mathrm{C}_{\mathrm{Me}}\end{array}$ & $\begin{array}{c}1.881 \\
1.897 ; 1.894 ; 1.895\end{array}$ & $\begin{array}{l}\mathrm{Ga}-\mathrm{C}_{10}-\mathrm{Si}_{13} \\
\mathrm{C}_{8}-\mathrm{Si}_{11}-\mathrm{C}_{\mathrm{Me}}\end{array}$ & $\begin{array}{c}122.5 \\
111.1 ; 110.1 ; 110.7\end{array}$ \\
\hline $\mathrm{Si}_{12}-\mathrm{C}_{\mathrm{Me}}$ & $1.894 ; 1.895 ; 1.898$ & $\mathrm{C}_{9}-\mathrm{Si}_{12}-\mathrm{C}_{\mathrm{Me}}$ & $111.3 ; 110.3 ; 110.7$ \\
\hline $\mathrm{Si}_{13}-\mathrm{C}_{\mathrm{Me}}$ & $1.894 ; 1.896 ; 1.896$ & $\mathrm{C}_{10}-\mathrm{Si}_{13}-\mathrm{C}_{\mathrm{Me}}$ & $110.5 ; 108.7 ; 111.7$ \\
\hline
\end{tabular}

5. Optimized geometry I $I^{t} B u+4$-bromobenzaldehyde 


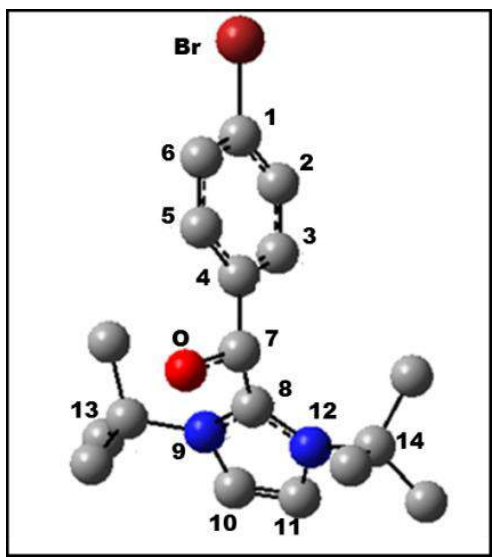

\begin{tabular}{|c|c|c|c|}
\hline \multicolumn{2}{|c|}{ Principal bond lengths $(\AA)$} & \multicolumn{2}{|c|}{ Principal bond angles $\left({ }^{\circ}\right)$} \\
\hline $\mathrm{C}_{1}-\mathrm{C}_{2}$ & 1.390 & $\mathrm{C}_{1}-\mathrm{C}_{2}-\mathrm{C}_{3}$ & 118.9 \\
\hline $\mathrm{C}_{2}-\mathrm{C}_{3}$ & 1.396 & $\mathrm{C}_{2}-\mathrm{C}_{3}-\mathrm{C}_{4}$ & 121.2 \\
\hline $\mathrm{C}_{3}-\mathrm{C}_{4}$ & 1.396 & $\mathrm{C}_{3}-\mathrm{C}_{4}-\mathrm{C}_{5}$ & 118.6 \\
\hline $\mathrm{C}_{4}-\mathrm{C}_{5}$ & 1.396 & $C_{4}-C_{5}-C_{6}$ & 121.2 \\
\hline $\mathrm{C}_{5}-\mathrm{C}_{6}$ & 1.392 & $\mathrm{C}_{5}-\mathrm{C}_{6}-\mathrm{C}_{1}$ & 119.0 \\
\hline $\mathrm{C}_{6}-\mathrm{C}_{1}$ & 1.392 & $\mathrm{C}_{6}-\mathrm{C}_{1}-\mathrm{C}_{2}$ & 121.2 \\
\hline $\mathrm{C}_{1}-\mathrm{Br}$ & 1.925 & $\mathrm{C}_{6}-\mathrm{C}_{1}-\mathrm{Br}$ & 119.5 \\
\hline $\mathrm{C}_{4}-\mathrm{C}_{7}$ & 1.556 & $\mathrm{C}_{3}-\mathrm{C}_{4}-\mathrm{C}_{7}$ & 124.9 \\
\hline $\mathrm{C}_{7}-\mathrm{H}$ & 1.110 & $\mathrm{C}_{4}-\mathrm{C}_{7}-\mathrm{H}$ & 100.8 \\
\hline $\mathrm{C}_{7}-\mathrm{O}$ & 1.329 & $\mathrm{C}_{4}-\mathrm{C}_{7}-\mathrm{O}$ & 117.1 \\
\hline $\mathrm{C}_{7}-\mathrm{C}_{8}$ & 1.555 & $\mathrm{C}_{7}-\mathrm{O}-\mathrm{C}_{8}$ & 112.8 \\
\hline $\mathrm{C}_{8}-\mathrm{N}_{9}$ & 1.365 & $\mathrm{C}_{7}-\mathrm{C}_{8}-\mathrm{N}_{9}$ & 127.5 \\
\hline $\mathrm{N}_{9}-\mathrm{C}_{10}$ & 1.385 & $\mathrm{C}_{8}-\mathrm{N}_{9}-\mathrm{C}_{10}$ & 108.5 \\
\hline $\mathrm{C}_{10}-\mathrm{C}_{11}$ & 1.347 & $N_{9}-C_{10}-C_{11}$ & 108.3 \\
\hline $\mathrm{C}_{11}-\mathrm{N}_{12}$ & 1.387 & $\mathrm{C}_{10}-\mathrm{C}_{11}-\mathrm{N}_{12}$ & 107.4 \\
\hline $\mathrm{N}_{12}-\mathrm{C}_{8}$ & 1.368 & $\mathrm{C}_{11}-\mathrm{N}_{12}-\mathrm{C}_{8}$ & 108.9 \\
\hline $\mathrm{N}_{9}-\mathrm{C}_{13}$ & 1.529 & $\mathrm{~N}_{12}-\mathrm{C}_{8}-\mathrm{N}_{9}$ & 106.9 \\
\hline $\mathrm{C}_{13}-\mathrm{C}_{\mathrm{Me}}$ & $1.539 ; 1.528 ; 1.538$ & $C_{10}-N_{9}-C_{13}$ & 120.0 \\
\hline $\mathrm{C}_{12}-\mathrm{C}_{14}$ & 1.518 & $\mathrm{~N}_{9}-\mathrm{C}_{13}-\mathrm{C}_{\mathrm{Me}}$ & $106.3 ; 108.4 ; 112.0$ \\
\hline $\mathrm{C}_{14}-\mathrm{C}_{\mathrm{Me}}$ & $1.544 ; 1.539 ; 1.539$ & $\mathrm{C}_{11}-\mathrm{N}_{12}-\mathrm{C}_{14}$ & 123.0 \\
\hline & & $\mathrm{N}_{12}-\mathrm{C}_{14}-\mathrm{C}_{\mathrm{Me}}$ & $109.1 ; 110.4 ; 109.1$ \\
\hline
\end{tabular}

6. Optimized geometry $a I^{\mathrm{t}} \mathrm{Bu}+4$-bromobenzaldehyde 


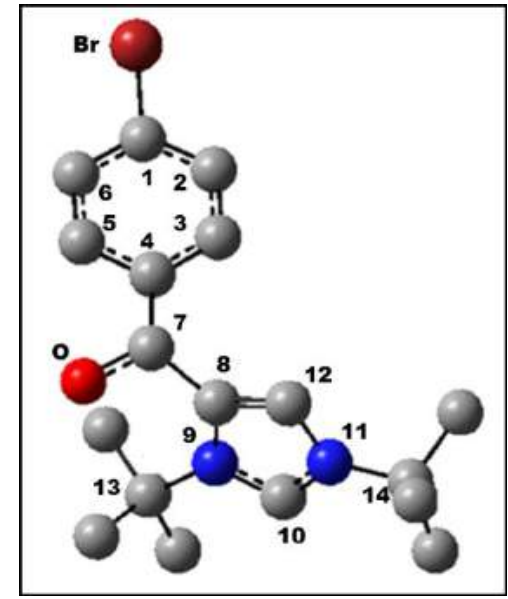

\begin{tabular}{|c|c|c|c|}
\hline \multicolumn{2}{|c|}{ Principal bond lengths $(\AA)$} & \multicolumn{2}{|c|}{ Principal bond angles $\left({ }^{\circ}\right)$} \\
\hline $\mathrm{C}_{1}-\mathrm{C}_{2}$ & 1.390 & $\mathrm{C}_{1}-\mathrm{C}_{2}-\mathrm{C}_{3}$ & 118.8 \\
\hline $\mathrm{C}_{2}-\mathrm{C}_{3}$ & 1.395 & $\mathrm{C}_{2}-\mathrm{C}_{3}-\mathrm{C}_{4}$ & 121.3 \\
\hline $\mathrm{C}_{3}-\mathrm{C}_{4}$ & 1.397 & $\mathrm{C}_{3}-\mathrm{C}_{4}-\mathrm{C}_{5}$ & 118.4 \\
\hline $\mathrm{C}_{4}-\mathrm{C}_{5}$ & 1.395 & $\mathrm{C}_{4}-\mathrm{C}_{5}-\mathrm{C}_{6}$ & 121.3 \\
\hline $\mathrm{C}_{5}-\mathrm{C}_{6}$ & 1.393 & $\mathrm{C}_{5}-\mathrm{C}_{6}-\mathrm{C}_{1}$ & 118.9 \\
\hline $\mathrm{C}_{6}-\mathrm{C}_{1}$ & 1.390 & $\mathrm{C}_{6}-\mathrm{C}_{1}-\mathrm{C}_{2}$ & 121.2 \\
\hline $\mathrm{C}_{1}-\mathrm{Br}$ & 1.928 & $\mathrm{C}_{6}-\mathrm{C}_{1}-\mathrm{Br}$ & 119.6 \\
\hline $\mathrm{C}_{4}-\mathrm{C}_{7}$ & 1.568 & $\mathrm{C}_{3}-\mathrm{C}_{4}-\mathrm{C}_{7}$ & 125.2 \\
\hline $\mathrm{C}_{7}-\mathrm{H}$ & 1.128 & $\mathrm{C}_{4}-\mathrm{C}_{7}-\mathrm{H}$ & 102.5 \\
\hline $\mathrm{C}_{7}-\mathrm{O}$ & 1.321 & $\mathrm{C}_{4}-\mathrm{C}_{7}-\mathrm{O}$ & 114.6 \\
\hline $\mathrm{C}_{7}-\mathrm{C}_{8}$ & 1.562 & $\mathrm{C}_{4}-\mathrm{C}_{7}-\mathrm{C}_{8}$ & 109.7 \\
\hline $\mathrm{C}_{8}-\mathrm{N}_{9}$ & 1.405 & $\mathrm{C}_{7}-\mathrm{C}_{8}-\mathrm{N}_{9}$ & 129.1 \\
\hline $\mathrm{N}_{9}-\mathrm{C}_{10}$ & 1.340 & $\mathrm{C}_{8}-\mathrm{N}_{9}-\mathrm{C}_{10}$ & 108.4 \\
\hline $\mathrm{C}_{10}-\mathrm{N}_{11}$ & 1.336 & $\mathrm{~N}_{9}-\mathrm{C}_{10}-\mathrm{N}_{11}$ & 110.1 \\
\hline $\mathrm{N}_{11}-\mathrm{C}_{12}$ & 1.385 & $\mathrm{C}_{10}-\mathrm{N}_{11}-\mathrm{C}_{12}$ & 107.0 \\
\hline $\mathrm{C}_{12}-\mathrm{C}_{8}$ & 1.368 & $\mathrm{~N}_{11}-\mathrm{C}_{12}-\mathrm{C}_{8}$ & 109.2 \\
\hline $\mathrm{N}_{9}-\mathrm{C}_{13}$ & 1.525 & $\mathrm{C}_{12}-\mathrm{C}_{8}-\mathrm{N}_{9}$ & 105.2 \\
\hline $\mathrm{C}_{13}-\mathrm{C}_{\mathrm{Me}}$ & $1.531 ; 1.537 ; 1.536$ & $\mathrm{C}_{10}-\mathrm{N}_{9}-\mathrm{C}_{13}$ & 122.2 \\
\hline $\mathrm{N}_{11}-\mathrm{C}_{14}$ & 1.499 & $\mathrm{~N}_{9}-\mathrm{C}_{13}-\mathrm{C}_{\mathrm{Me}}$ & $109.1 ; 107.0 ; 109.0$ \\
\hline \multirow[t]{2}{*}{$\mathrm{C}_{14}-\mathrm{C}_{\mathrm{Me}}$} & $1.533 ; 1.537 ; 1.538$ & $\mathrm{C}_{10}-\mathrm{N}_{11}-\mathrm{C}_{14}$ & 127.4 \\
\hline & & $\mathrm{N}_{11}-\mathrm{C}_{14}-\mathrm{C}_{\mathrm{Me}}$ & $108.1 ; 109.8 ; 108.1$ \\
\hline
\end{tabular}

7. Optimized geometry of $\mathbf{I I}_{\mathrm{Ar}}$ 


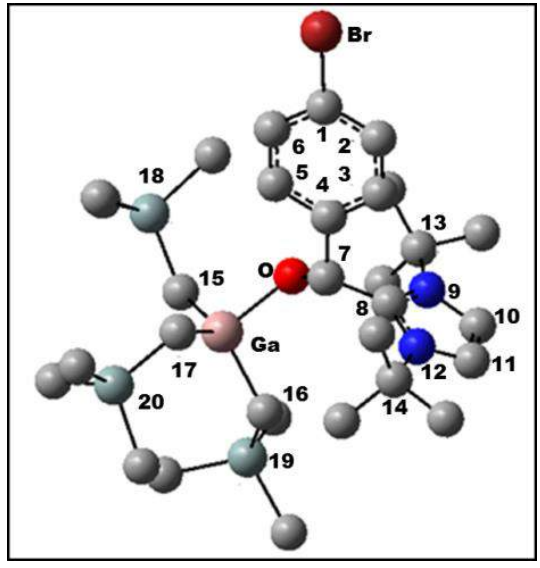

\begin{tabular}{|c|c|c|c|c|c|}
\hline \multicolumn{2}{|c|}{ Principal bond lengths $(\AA)$} & \multicolumn{2}{|c|}{ Principal bond angles $\left({ }^{\circ}\right)$} & \multicolumn{2}{|c|}{ Principal Bond Indices } \\
\hline $\mathrm{C}_{1}-\mathrm{C}_{2}$ & 1.389 & $\mathrm{C}_{1}-\mathrm{C}_{2}-\mathrm{C}_{3}$ & 119.0 & $\mathrm{C}_{1}-\mathrm{C}_{2}$ & 1.41 \\
\hline $\mathrm{C}_{2}-\mathrm{C}_{3}$ & 1.395 & $\mathrm{C}_{2}-\mathrm{C}_{3}-\mathrm{C}_{4}$ & 121.3 & $\mathrm{C}_{2}-\mathrm{C}_{3}$ & 1.41 \\
\hline $\mathrm{C}_{3}-\mathrm{C}_{4}$ & 1.396 & $\mathrm{C}_{3}-\mathrm{C}_{4}-\mathrm{C}_{5}$ & 118.3 & $\mathrm{C}_{3}-\mathrm{C}_{4}$ & 1.41 \\
\hline $\mathrm{C}_{4}-\mathrm{C}_{5}$ & 1.397 & $\mathrm{C}_{4}-\mathrm{C}_{5}-\mathrm{C}_{6}$ & 121.2 & $\mathrm{C}_{4}-\mathrm{C}_{5}$ & 1.39 \\
\hline $\mathrm{C}_{5}-\mathrm{C}_{6}$ & 1.391 & $\mathrm{C}_{5}-\mathrm{C}_{6}-\mathrm{C}_{1}$ & 119.2 & $\mathrm{C}_{5}-\mathrm{C}_{6}$ & 1.43 \\
\hline $\mathrm{C}_{6}-\mathrm{C}_{1}$ & 1.392 & $\mathrm{C}_{6}-\mathrm{C}_{1}-\mathrm{C}_{2}$ & 120.9 & $\mathrm{C}_{6}-\mathrm{C}_{1}$ & 1.40 \\
\hline $\mathrm{C}_{1}-\mathrm{Br}$ & 1.919 & $\mathrm{C}_{6}-\mathrm{C}_{1}-\mathrm{Br}$ & 119.6 & $\mathrm{C}_{1}-\mathrm{Br}$ & 1.04 \\
\hline $\mathrm{C}_{4}-\mathrm{C}_{7}$ & 1.547 & $\mathrm{C}_{3}-\mathrm{C}_{4}-\mathrm{C}_{7}$ & 124.6 & $\mathrm{C}_{4}-\mathrm{C}_{7}$ & 0.96 \\
\hline $\mathrm{C}_{7}-\mathrm{H}$ & 1.098 & $\mathrm{C}_{4}-\mathrm{C}_{7}-\mathrm{H}$ & 104.6 & $\mathrm{C}_{7}-\mathrm{H}$ & 0.87 \\
\hline $\mathrm{C}_{7}-\mathrm{O}$ & 1.369 & $\mathrm{C}_{4}-\mathrm{C}_{7}-\mathrm{O}$ & 113.2 & $\mathrm{C}_{7}-\mathrm{O}$ & 1.02 \\
\hline $\mathrm{C}_{7}-\mathrm{C}_{8}$ & 1.547 & $\mathrm{C}_{4}-\mathrm{C}_{7}-\mathrm{C}_{8}$ & 111.9 & $\mathrm{C}_{7}-\mathrm{C}_{8}$ & 0.96 \\
\hline $\mathrm{C}_{8}-\mathrm{N}_{9}$ & 1.362 & $\mathrm{C}_{7}-\mathrm{C}_{8}-\mathrm{N}_{9}$ & 127.4 & $\mathrm{C}_{8}-\mathrm{N}_{9}$ & 1.30 \\
\hline $\mathrm{N}_{9}-\mathrm{C}_{10}$ & 1.382 & $\mathrm{C}_{8}-\mathrm{N}_{9}-\mathrm{C}_{10}$ & 108.2 & $\mathrm{~N}_{9}-\mathrm{C}_{10}$ & 1.15 \\
\hline $\mathrm{C}_{10}-\mathrm{C}_{11}$ & 1.348 & $\mathrm{~N}_{9}-\mathrm{C}_{10}-\mathrm{C}_{11}$ & 108.2 & $\mathrm{C}_{10}-\mathrm{C}_{11}$ & 1.63 \\
\hline $\mathrm{C}_{11}-\mathrm{N}_{12}$ & 1.383 & $\mathrm{C}_{10}-\mathrm{C}_{11}-\mathrm{N}_{12}$ & 107.6 & $\mathrm{C}_{11}-\mathrm{N}_{12}$ & 1.14 \\
\hline $\mathrm{N}_{12}-\mathrm{C}_{8}$ & 1.366 & $\mathrm{C}_{11}-\mathrm{N}_{12}-\mathrm{C}_{8}$ & 108.4 & $\mathrm{~N}_{12}-\mathrm{C}_{8}$ & 1.28 \\
\hline$N_{9}-C_{13}$ & 1.540 & $\mathrm{~N}_{12}-\mathrm{C}_{8}-\mathrm{N}_{9}$ & 107.8 & $N_{9}-C_{13}$ & 0.84 \\
\hline $\mathrm{N}_{12}-\mathrm{C}_{14}$ & 1.532 & $\mathrm{C}_{10}-\mathrm{N}_{9}-\mathrm{C}_{13}$ & 121.1 & $\mathrm{~N}_{12}-\mathrm{C}_{14}$ & 0.85 \\
\hline $\mathrm{C}_{13}-\mathrm{C}_{\mathrm{Me}}$ & $1.540 ; 1.529 ; 1.534$ & $\mathrm{~N}_{9}-\mathrm{C}_{13}-\mathrm{C}_{\mathrm{Me}}$ & $107.5 ; 108.0 ; 111.3$ & $\mathrm{C}_{13}-\mathrm{C}_{\mathrm{Me}}$ & $1.00 ; 1.00 ; 1.01$ \\
\hline $\mathrm{C}_{14}-\mathrm{C}_{\mathrm{Me}}$ & $1.539 ; 1.536 ; 1.536$ & $\mathrm{C}_{11}-\mathrm{N}_{12}-\mathrm{C}_{14}$ & 122.0 & $\mathrm{C}_{14}-\mathrm{C}_{\mathrm{Me}}$ & $1.00 ; 1.00 ; 1.00$ \\
\hline & & $\mathrm{N}_{12}-\mathrm{C}_{14}-\mathrm{C}_{\mathrm{Me}}$ & $108.9 ; 110.1 ; 109.4$ & & \\
\hline O-Ga & 2.016 & $\mathrm{C}_{7}-\mathrm{O}-\mathrm{Ga}$ & 120.2 & O-Ga & 0.33 \\
\hline $\mathrm{Ga} \mathrm{C}_{15}$ & 2.027 & O-Ga-C ${ }_{15}$ & 105.4 & Ga-C ${ }_{15}$ & 0.56 \\
\hline $\mathrm{Ga} \mathrm{C}_{16}$ & 2.058 & O-Ga-C ${ }_{16}$ & 103.5 & $\mathrm{Ga}-\mathrm{C}_{16}$ & 0.59 \\
\hline Ga-C ${ }_{17}$ & 2.046 & O-Ga-C ${ }_{17}$ & 102.4 & Ga-C ${ }_{17}$ & 0.57 \\
\hline $\mathrm{C}_{15}-\mathrm{Si}_{18}$ & 1.874 & $\mathbf{G a}-\mathrm{C}_{15}-\mathrm{Si}_{18}$ & 121.9 & $\mathrm{C}_{15}-\mathrm{Si}_{18}$ & 0.83 \\
\hline $\mathrm{C}_{16}-\mathrm{Si}_{19}$ & 1.875 & $\mathrm{Ga}-\mathrm{C}_{16}-\mathrm{Si}_{19}$ & 123.7 & $\mathrm{C}_{16}-\mathrm{Si}_{19}$ & 0.83 \\
\hline
\end{tabular}




\begin{tabular}{|c|c|c|c|c|c|}
\hline $\mathrm{C}_{17}-\mathrm{Si}_{20}$ & 1.875 & $\mathbf{G a}-\mathrm{C}_{17}-\mathrm{Si}_{20}$ & 118.9 & $\mathrm{C}_{17}-\mathrm{Si}_{20}$ & 0.83 \\
\hline $\mathrm{Si}_{18}-\mathrm{C}_{\mathrm{Me}}$ & $1.896 ; 1.902 ; 1.897$ & $\mathrm{C}_{15}-\mathrm{Si}_{18}-\mathrm{C}_{\mathrm{Me}}$ & $111.8 ; 111.2 ; 111.2$ & $\mathrm{Si}_{18}-\mathrm{C}_{M e}$ & $0.80 ; 0.81 ; 0.81$ \\
\hline $\mathrm{Si}_{19}-\mathrm{C}_{\mathrm{Me}}$ & $1.904 ; 1.895 ; 1.898$ & $\mathrm{C}_{16}-\mathrm{Si}_{19}-\mathrm{C}_{\mathrm{Me}}$ & $112.2 ; 114.1 ; 109.4$ & $\mathrm{Si}_{19}-\mathrm{C}_{\mathrm{Me}}$ & $0.81 ; 0.81 ; 0.81$ \\
\hline $\mathrm{Si}_{20}-\mathrm{C}_{\mathrm{Me}}$ & $1.899 ; 1.901 ; 1.894$ & $\mathrm{C}_{17}-\mathrm{Si}_{20}-\mathrm{C}_{\mathrm{Me}}$ & $110.3 ; 111.5 ; 112.7$ & $\mathrm{Si}_{20}-\mathrm{C}_{\mathrm{Me}}$ & $0.81 ; 0.81 ; 0.82$ \\
\hline
\end{tabular}
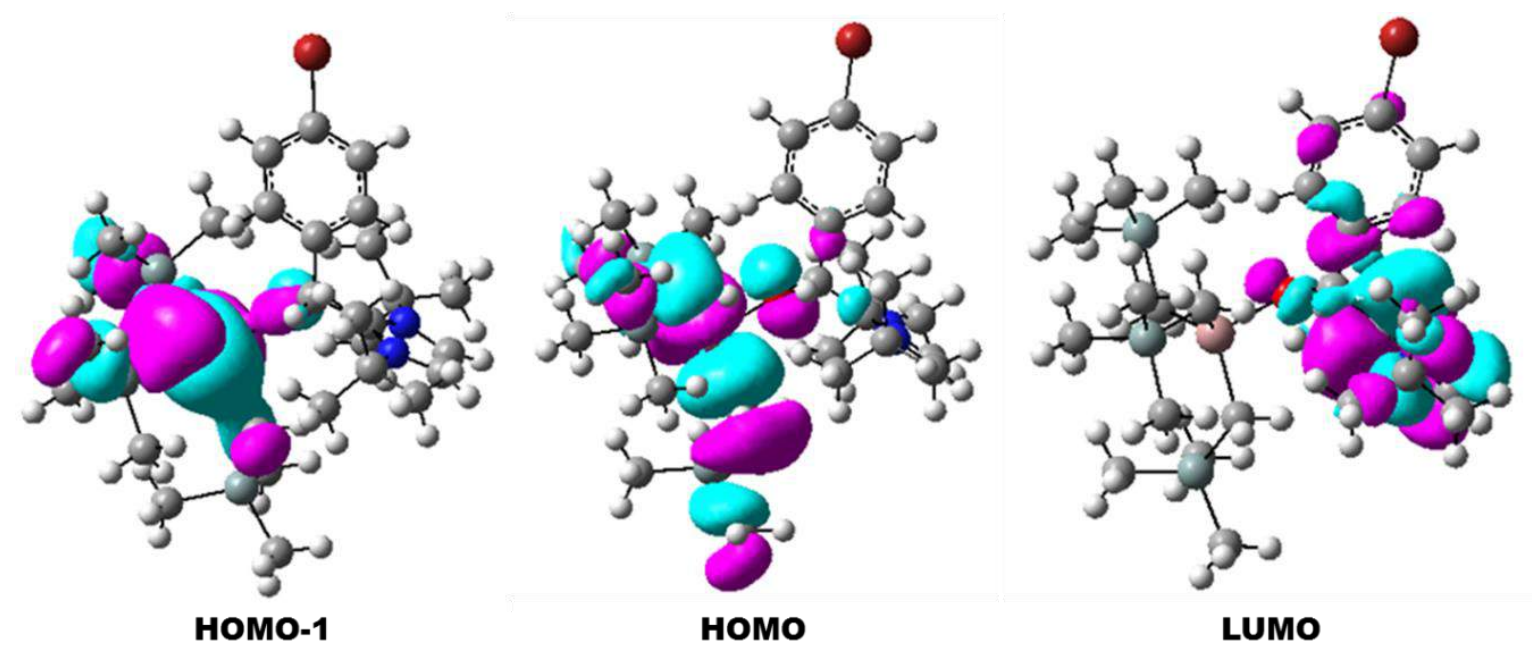

Figure S20: Representation of molecular orbitals HOMO-1, HOMO and LUMO of $\mathbf{I I}_{\mathbf{A r}}$.

8. Optimized geometry of $\mathrm{III}_{\mathrm{Ar}}$

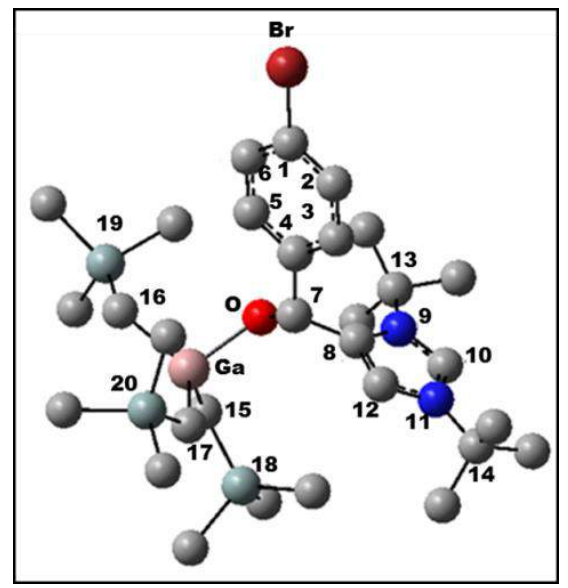

\begin{tabular}{|c|c|c|c|c|c|}
\hline \multicolumn{2}{|c|}{ Principal bond lengths $(\AA)$} & \multicolumn{2}{|c|}{ Principal bond angles $\left({ }^{\circ}\right)$} & \multicolumn{2}{|c|}{ Principal Bond Indices } \\
\hline $\mathrm{C}_{1}-\mathrm{C}_{2}$ & 1.391 & $\mathrm{C}_{1}-\mathrm{C}_{2}-\mathrm{C}_{3}$ & 119.0 & $\mathrm{C}_{1}-\mathrm{C}_{2}$ & 1.41 \\
\hline $\mathrm{C}_{2}-\mathrm{C}_{3}$ & 1.394 & $\mathrm{C}_{2}-\mathrm{C}_{3}-\mathrm{C}_{4}$ & 121.2 & $\mathrm{C}_{2}-\mathrm{C}_{3}$ & 1.43 \\
\hline $\mathrm{C}_{3}-\mathrm{C}_{4}$ & 1.397 & $\mathrm{C}_{3}-\mathrm{C}_{4}-\mathrm{C}_{5}$ & 118.5 & $\mathrm{C}_{3}-\mathrm{C}_{4}$ & 1.40 \\
\hline $\mathrm{C}_{4}-\mathrm{C}_{5}$ & 1.395 & $\mathrm{C}_{4}-\mathrm{C}_{5}-\mathrm{C}_{6}$ & 121.1 & $\mathrm{C}_{4}-\mathrm{C}_{5}$ & 1.40 \\
\hline $\mathrm{C}_{5}-\mathrm{C}_{6}$ & 1.393 & $\mathrm{C}_{5}-\mathrm{C}_{6}-\mathrm{C}_{1}$ & 119.2 & $\mathrm{C}_{5}-\mathrm{C}_{6}$ & 1.41 \\
\hline $\mathrm{C}_{6}-\mathrm{C}_{1}$ & 1.390 & $\mathrm{C}_{6}-\mathrm{C}_{1}-\mathrm{C}_{2}$ & 121.0 & $\mathrm{C}_{6}-\mathrm{C}_{1}$ & 1.41 \\
\hline $\mathrm{C}_{1}-\mathrm{Br}$ & 1.921 & $\mathrm{C}_{6}-\mathrm{C}_{1}-\mathrm{Br}$ & 119.6 & $\mathrm{C}_{1}-\mathrm{Br}$ & 1.03 \\
\hline
\end{tabular}




\begin{tabular}{|c|c|c|c|c|c|}
\hline $\mathrm{C}_{4}-\mathrm{C}_{7}$ & 1.545 & $\mathrm{C}_{3}-\mathrm{C}_{4}-\mathrm{C}_{7}$ & 122.6 & $\mathrm{C}_{4}-\mathrm{C}_{7}$ & 0.96 \\
\hline $\mathrm{C}_{7}-\mathrm{H}$ & 1.105 & $\mathrm{C}_{4}-\mathrm{C}_{7}-\mathrm{H}$ & 104.1 & $\mathrm{C}_{7}-\mathrm{H}$ & 0.87 \\
\hline $\mathrm{C}_{7}-\mathrm{O}$ & 1.370 & $\mathrm{C}_{4}-\mathrm{C}_{7}-\mathrm{O}$ & 114.1 & $\mathrm{C}_{7}-\mathrm{O}$ & 1.02 \\
\hline $\mathrm{C}_{7}-\mathrm{C}_{8}$ & 1.532 & $\mathrm{C}_{4}-\mathrm{C}_{7}-\mathrm{C}_{8}$ & 112.6 & $\mathrm{C}_{7}-\mathrm{C}_{8}$ & 0.97 \\
\hline $\mathrm{C}_{8}-\mathrm{N}_{9}$ & 1.405 & $\mathrm{C}_{7}-\mathrm{C}_{8}-\mathrm{N}_{9}$ & 130.6 & $\mathrm{C}_{8}-\mathrm{N}_{9}$ & 1.12 \\
\hline$N_{9}-C_{10}$ & 1.341 & $\mathrm{C}_{8}-\mathrm{N}_{9}-\mathrm{C}_{10}$ & 107.7 & $\mathrm{~N}_{9}-\mathrm{C}_{10}$ & 1.32 \\
\hline $\mathrm{C}_{10}-\mathrm{N}_{11}$ & 1.334 & $N_{9}-C_{10}-N_{11}$ & 110.4 & $\mathrm{C}_{10}-\mathrm{N}_{11}$ & 1.31 \\
\hline $\mathrm{N}_{11}-\mathrm{C}_{12}$ & 1.309 & $\mathrm{C}_{10}-\mathrm{N}_{11}-\mathrm{C}_{12}$ & 107.1 & $\mathrm{~N}_{11}-\mathrm{C}_{12}$ & 1.13 \\
\hline $\mathrm{C}_{12}-\mathrm{C}_{8}$ & 1.365 & $\mathrm{~N}_{11}-\mathrm{C}_{12}-\mathrm{C}_{8}$ & 109.1 & $\mathrm{~N}_{12}-\mathrm{C}_{8}$ & 1.59 \\
\hline $\mathrm{N}_{9}-\mathrm{C}_{13}$ & 1.529 & $\mathrm{~N}_{12}-\mathrm{C}_{8}-\mathrm{N}_{9}$ & 105.7 & $\mathrm{~N}_{9}-\mathrm{C}_{13}$ & 0.85 \\
\hline $\mathrm{C}_{12}-\mathrm{C}_{14}$ & 1.504 & $\mathrm{C}_{10}-\mathrm{N}_{9}-\mathrm{C}_{13}$ & 121.6 & $\mathrm{~N}_{11}-\mathrm{C}_{14}$ & 0.87 \\
\hline $\mathrm{C}_{13}-\mathrm{C}_{\mathrm{Me}}$ & $1.537 ; 1.530 ; 1.534$ & $\mathrm{~N}_{9}-\mathrm{C}_{13}-\mathrm{C}_{\mathrm{Me}}$ & $108.7 ; 109.8 ; 107.5$ & $\mathrm{C}_{13}-\mathrm{C}_{\mathrm{Me}}$ & $1.00 ; 1.00 ; 1.01$ \\
\hline \multirow[t]{2}{*}{$\mathrm{C}_{14}-\mathrm{C}_{\mathrm{Me}}$} & \multirow{2}{*}{$1.533 ; 1.537 ; 1.537$} & $\mathrm{C}_{10}-\mathrm{N}_{11}-\mathrm{C}_{14}$ & 127.5 & \multirow[t]{2}{*}{$\mathrm{C}_{14}-\mathrm{C}_{\mathrm{Me}}$} & \multirow[t]{2}{*}{$1.00 ; 1.00 ; 1.00$} \\
\hline & & $\mathrm{N}_{11}-\mathrm{C}_{14}-\mathrm{C}_{\mathrm{Me}}$ & $107.9 ; 107.9 ; 109.5$ & & \\
\hline O-Ga & 2.000 & $\mathrm{C}_{7}-\mathrm{O}-\mathrm{Ga}$ & 124.0 & O-Ga & 0.36 \\
\hline Ga-C $C_{15}$ & 2.048 & O-Ga-C ${ }_{15}$ & 103.8 & Ga-C 15 & 0.60 \\
\hline Ga-C ${ }_{16}$ & 2.039 & O-Ga-C ${ }_{16}$ & 103.9 & Ga-C ${ }_{16}$ & 0.60 \\
\hline $\mathrm{Ga}^{-\mathrm{C}_{17}}$ & 2.056 & O-Ga-C ${ }_{17}$ & 105.8 & $\mathbf{G a} \mathrm{C}_{17}$ & 0.59 \\
\hline $\mathrm{C}_{15}-\mathrm{Si}_{18}$ & 1.867 & $\mathrm{Ga}-\mathrm{C}_{15}-\mathrm{Si}_{18}$ & 123.9 & $\mathrm{C}_{15}-\mathrm{Si}_{18}$ & 0.85 \\
\hline $\mathrm{C}_{16}-\mathrm{Si}_{19}$ & 1.873 & Ga-C ${ }_{16}-\mathrm{Si}_{19}$ & 122.2 & $\mathrm{C}_{16}-\mathrm{Si}_{19}$ & 0.84 \\
\hline $\mathrm{C}_{17}-\mathrm{Si}_{20}$ & 1.874 & $\mathbf{G a}-\mathrm{C}_{17}-\mathrm{Si}_{20}$ & 124.0 & $\mathrm{C}_{17}-\mathrm{Si}_{20}$ & 0.84 \\
\hline $\mathrm{Si}_{18}-\mathrm{C}_{\mathrm{Me}}$ & $1.897 ; 1.904 ; 1.906$ & $\mathrm{C}_{15}-\mathrm{Si}_{18}-\mathrm{C}_{\mathrm{Me}}$ & $113.4 ; 111.4 ; 111.2$ & $\mathrm{Si}_{18}-\mathrm{C}_{\mathrm{Me}}$ & $0.80 ; 0.80 ; 0.82$ \\
\hline $\mathrm{Si}_{19}-\mathrm{C}_{\mathrm{Me}}$ & $1.900 ; 1.902 ; 1.897$ & $\mathrm{C}_{16}-\mathrm{Si}_{19}-\mathrm{C}_{\mathrm{Me}}$ & $110.7 ; 111.3 ; 112.8$ & $\mathrm{Si}_{19}-\mathrm{C}_{\mathrm{Me}}$ & $0.81 ; 0.81 ; 0.81$ \\
\hline $\mathrm{Si}_{20}-\mathrm{C}_{\mathrm{Me}}$ & $1.897 ; 1.905 ; 1.894$ & $\mathrm{C}_{17}-\mathrm{Si}_{20}-\mathrm{C}_{\mathrm{Me}}$ & $113.0 ; 110.9 ; 110.5$ & $\mathrm{Si}_{20}-\mathrm{C}_{\mathrm{Me}}$ & $0.80 ; 0.81 ; 0.82$ \\
\hline
\end{tabular}
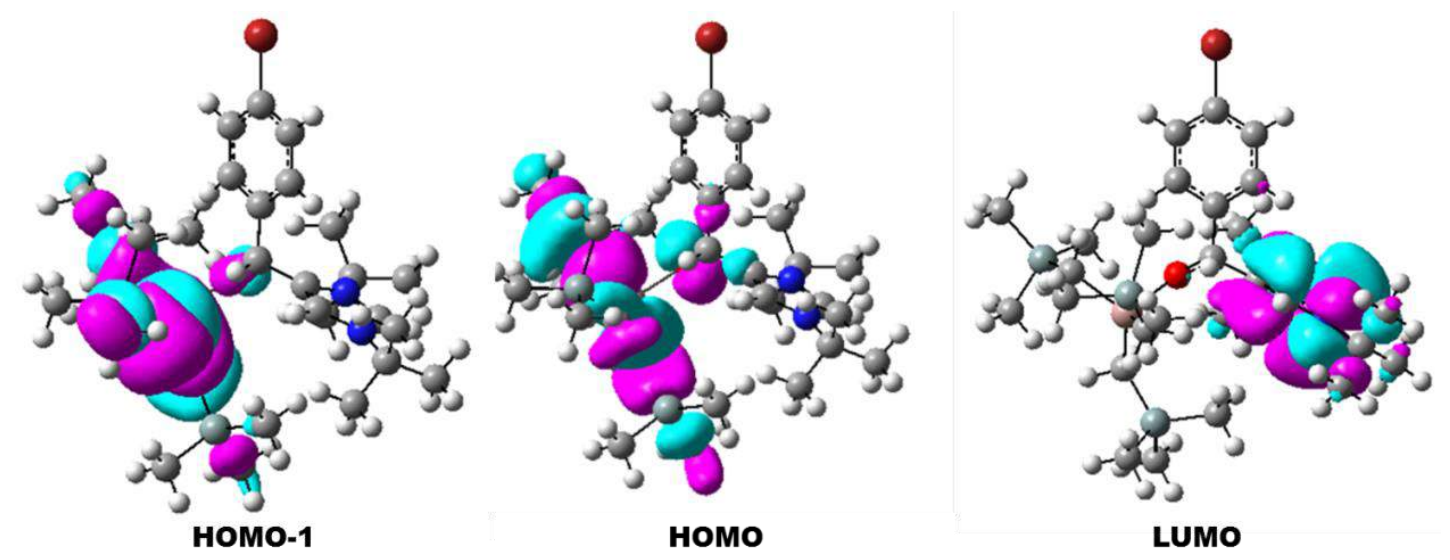

Figure S21: Representation of molecular orbitals HOMO-1, HOMO and LUMO of $\mathbf{I I I}_{\mathrm{Ar}}$.

9. Optimized geometry of $\|_{H}$ 


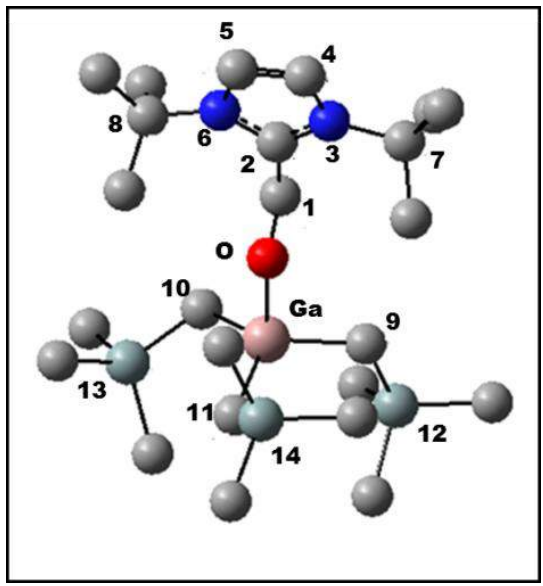

\begin{tabular}{|c|c|c|c|}
\hline \multicolumn{2}{|c|}{ Principal bond lengths $(\AA)$} & \multicolumn{2}{|c|}{ Principal bond angles $\left({ }^{\circ}\right)$} \\
\hline $\mathrm{C}_{1}-\mathrm{C}_{2}$ & 1.519 & $\mathrm{C}_{1}-\mathrm{C}_{2}-\mathrm{N}_{3}$ & 126.2 \\
\hline $\mathrm{C}_{2}-\mathrm{N}_{3}$ & 1.359 & $\mathrm{C}_{2}-\mathrm{N}_{3}-\mathrm{C}_{4}$ & 108.8 \\
\hline $\mathrm{N}_{3}-\mathrm{C}_{4}$ & 1.382 & $\mathrm{~N}_{3}-\mathrm{C}_{4}-\mathrm{C}_{5}$ & 107.2 \\
\hline $\mathrm{C}_{4}-\mathrm{C}_{5}$ & 1.351 & $\mathrm{C}_{4}-\mathrm{C}_{5}-\mathrm{N}_{6}$ & 110.1 \\
\hline $\mathrm{C}_{5}-\mathrm{N}_{6}$ & 1.387 & $\mathrm{C}_{5}-\mathrm{N}_{6}-\mathrm{C}_{2}$ & 108.0 \\
\hline $\mathrm{N}_{6}-\mathrm{C}_{2}$ & 1.359 & $\mathrm{~N}_{6}-\mathrm{C}_{2}-\mathrm{N}_{3}$ & 105.9 \\
\hline $\mathrm{N}_{3}-\mathrm{C}_{7}$ & 1.524 & $\mathrm{C}_{2}-\mathrm{N}_{3}-\mathrm{C}_{7}$ & 125.4 \\
\hline $\mathrm{N}_{6}-\mathrm{C}_{8}$ & 1.526 & $\mathrm{~N}_{3}-\mathrm{C}_{7}-\mathrm{C}_{\mathrm{Me}}$ & $107.9 ; 108.0 ; 109.5$ \\
\hline $\mathrm{C}_{7}-\mathrm{C}_{\mathrm{Me}}$ & $1.537 ; 1.539 ; 1.535$ & $\mathrm{C}_{2}-\mathrm{N}_{6}-\mathrm{C}_{8}$ & 129.1 \\
\hline $\mathrm{C}_{8}-\mathrm{C}_{\mathrm{Me}}$ & $1.537 ; 1.539 ; 1.535$ & $\mathrm{~N}_{6}-\mathrm{C}_{8}-\mathrm{C}_{\mathrm{Me}}$ & $108.6 ; 107.7 ; 109.5$ \\
\hline $\mathrm{C}_{1}-\mathrm{O}$ & 1.375 & $\mathrm{C}_{2}-\mathrm{C}_{1}-\mathrm{O}$ & 111.8 \\
\hline $\mathrm{C}_{1}-\mathrm{H}$ & $1.100 ; 1.099$ & $\mathrm{H}-\mathrm{C}_{1}-\mathrm{O}$ & $112.9 ; 113.9$ \\
\hline O-Ga & 2.010 & $\mathrm{H}-\mathrm{C}_{1}-\mathrm{H}$ & 105.1 \\
\hline $\mathrm{Ga}-\mathrm{C}_{9}$ & 2.045 & $\mathrm{C}_{1}-\mathrm{O}-\mathrm{Ga}$ & 143.3 \\
\hline Ga-C ${ }_{10}$ & 2.054 & O-Ga- $\mathrm{C}_{9}$ & 101.3 \\
\hline Ga-C ${ }_{11}$ & 2.028 & O-Ga- $\mathrm{C}_{10}$ & 102.4 \\
\hline $\begin{array}{l}\mathrm{C}_{9}-\mathrm{Si}_{12} \\
\mathrm{C}_{10}-\mathrm{Si}_{13}\end{array}$ & $\begin{array}{l}1.872 \\
1.873\end{array}$ & $\begin{array}{l}\text { O-Ga-C } C_{11} \\
\text { Ga-C } C_{9-} \mathrm{Si}_{12}\end{array}$ & $\begin{array}{l}103.2 \\
121.9\end{array}$ \\
\hline $\mathrm{C}_{11}-\mathrm{Si}_{14}$ & 1.871 & Ga- $\mathbf{C}_{10}-\mathrm{Si}_{13}$ & 121.5 \\
\hline $\mathrm{Si}_{13}-\mathrm{C}_{\mathrm{Me}}$ & $1.903 ; 1.898 ; 1.896$ & Ga- $\mathbf{C}_{11}-S_{14}$ & 121.5 \\
\hline $\mathrm{Si}_{14}-\mathrm{C}_{\mathrm{Me}}$ & $1.895 ; 1.900 ; 1.903$ & $\mathrm{C}_{9}-\mathrm{Si}_{12}-\mathrm{C}_{\mathrm{Me}}$ & $111.3 ; 110.7 ; 111.8$ \\
\hline $\mathrm{Si}_{15}-\mathrm{C}_{\mathrm{Me}}$ & $1.903 ; 1.897 ; 1.898$ & $\mathrm{C}_{10}-\mathrm{Si}_{13}-\mathrm{C}_{\mathrm{Me}}$ & $112.6 ; 110.8 ; 110.8$ \\
\hline & & $\mathrm{C}_{11}-\mathrm{Si}_{14}-\mathrm{C}_{\mathrm{Me}}$ & $112.0 ; 108.5 ; 106.7$ \\
\hline
\end{tabular}

10. Optimized geometry of $\mathrm{III}_{\mathrm{H}}$ 


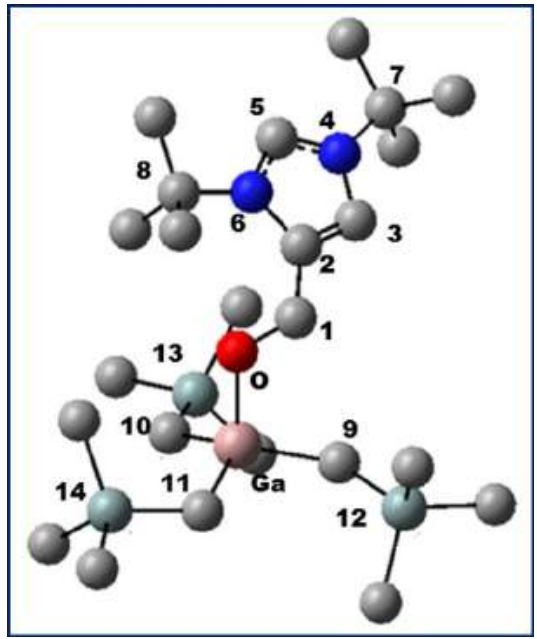

\begin{tabular}{|c|c|c|c|}
\hline \multicolumn{2}{|c|}{ Principal bond lengths (̊) } & \multicolumn{2}{|c|}{ Principal bond angles $\left({ }^{\circ}\right)$} \\
\hline $\mathrm{C}_{1}-\mathrm{C}_{2}$ & 1.516 & $\mathrm{C}_{1}-\mathrm{C}_{2}-\mathrm{C}_{3}$ & 126.2 \\
\hline $\mathrm{C}_{2}-\mathrm{C}_{3}$ & 1.364 & $\mathrm{C}_{2}-\mathrm{C}_{3}-\mathrm{N}_{4}$ & 108.8 \\
\hline $\mathrm{C}_{3}-\mathrm{N}_{4}$ & 1.383 & $\mathrm{C}_{3}-\mathrm{N}_{4}-\mathrm{C}_{5}$ & 107.2 \\
\hline $\mathrm{N}_{4}-\mathrm{C}_{5}$ & 1.335 & $\mathrm{~N}_{4}-\mathrm{C}_{5}-\mathrm{N}_{6}$ & 110.1 \\
\hline $\mathrm{C}_{5}-\mathrm{N}_{6}$ & 1.340 & $\mathrm{C}_{5}-\mathrm{N}_{6}-\mathrm{C}_{2}$ & 108.0 \\
\hline $\mathrm{N}_{6}-\mathrm{C}_{2}$ & 1.401 & $\mathrm{~N}_{6}-\mathrm{C}_{2}-\mathrm{C}_{3}$ & 105.9 \\
\hline $\mathrm{N}_{4}-\mathrm{C}_{7}$ & 1.503 & $\mathrm{C}_{3}-\mathrm{N}_{3}-\mathrm{C}_{7}$ & 125.4 \\
\hline $\mathrm{N}_{6}-\mathrm{C}_{8}$ & 1.525 & $\mathrm{~N}_{4}-\mathrm{C}_{7}-\mathrm{C}_{\mathrm{Me}}$ & $107.9 ; 108.0 ; 109.5$ \\
\hline $\mathrm{C}_{7}-\mathrm{C}_{\mathrm{Me}}$ & $1.537 ; 1.537 ; 1.533$ & $\mathrm{C}_{2}-\mathrm{N}_{6}-\mathrm{C}_{8}$ & 129.1 \\
\hline $\mathrm{C}_{8}-\mathrm{C}_{\mathrm{Me}}$ & $1.537 ; 1.533 ; 1.535$ & $\mathrm{~N}_{6}-\mathrm{C}_{8}-\mathrm{C}_{\mathrm{Me}}$ & $108.6 ; 107.7 ; 109.5$ \\
\hline $\mathrm{C}_{1}-\mathrm{O}$ & 1.374 & $\mathrm{C}_{2}-\mathrm{C}_{1}-\mathrm{O}$ & 111.8 \\
\hline $\mathrm{C}_{1}-\mathrm{H}$ & $1.106 ; 1.101$ & $\mathrm{H}-\mathrm{C}_{1}-\mathrm{O}$ & $112.9 ; 113.9$ \\
\hline O-Ga & 1.979 & $\mathrm{H}-\mathrm{C}_{1}-\mathrm{H}$ & 105.1 \\
\hline Ga-C & 2.056 & $\mathrm{C}_{1}-\mathrm{O}-\mathrm{Ga}$ & 124.1 \\
\hline Ga-C 10 & 2.046 & O-Ga-C $C_{9}$ & 105.8 \\
\hline Ga-C ${ }_{11}$ & 2.041 & O-Ga- $\mathrm{C}_{10}$ & 102.3 \\
\hline $\begin{array}{l}\mathrm{C}_{9}-\mathrm{Si}_{12} \\
\mathrm{C}_{10}-\mathrm{Si}_{13}\end{array}$ & $\begin{array}{l}1.871 \\
1.866\end{array}$ & $\begin{array}{l}\text { O-Ga-C } C_{11} \\
\text { Ga-C }{ }_{9-S i_{12}}\end{array}$ & $\begin{array}{l}105.4 \\
123.3\end{array}$ \\
\hline $\mathrm{C}_{11}-\mathrm{Si}_{14}$ & 1.869 & $\mathbf{G a}-\mathrm{C}_{10}-\mathrm{Si}_{13}$ & 123.0 \\
\hline $\mathrm{Si}_{12}-\mathrm{C}_{\mathrm{Me}}$ & $1.901 ; 1.905 ; 1.895$ & Ga- $\mathrm{C}_{11-} \mathrm{Si}_{14}$ & 122.0 \\
\hline $\mathrm{Si}_{13}-\mathrm{C}_{\mathrm{Me}}$ & $1.904 ; 1.897 ; 1.906$ & $\mathrm{C}_{9}-\mathrm{Si}_{12}-\mathrm{C}_{\mathrm{Me}}$ & $113.1 ; 110.9 ; 110.9$ \\
\hline $\mathrm{Si}_{14}-\mathrm{C}_{\mathrm{Me}}$ & $1.904 ; 1.897 ; 1.899$ & $\mathrm{C}_{10}-\mathrm{Si}_{13}-\mathrm{C}_{\mathrm{Me}}$ & $112.6 ; 111.9 ; 111.4$ \\
\hline & & $\mathrm{C}_{11 .}-\mathrm{Si}_{14}-\mathrm{C}_{\mathrm{Me}}$ & $111.0 ; 112.2 ; 111.5$ \\
\hline
\end{tabular}

11. Optimized geometry of $\mathrm{GaR}_{3}+\alpha, \alpha, \alpha$-trifluoroacetophenone 


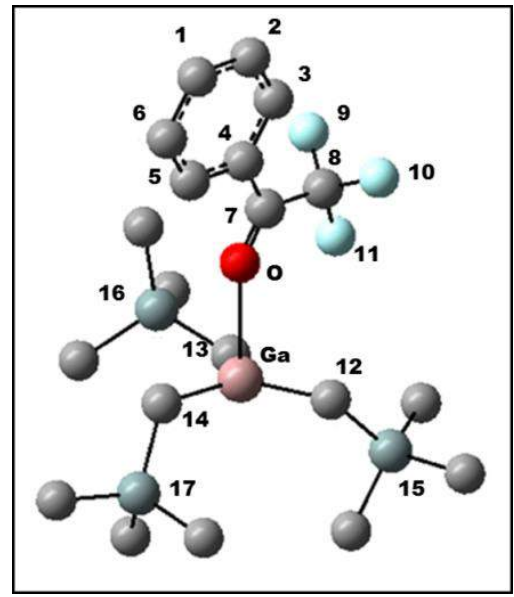

\begin{tabular}{|c|c|c|c|}
\hline \multicolumn{2}{|c|}{ Principal bond lengths $(\AA)$} & \multicolumn{2}{|c|}{ Principal bond angles $\left({ }^{\circ}\right)$} \\
\hline $\mathrm{C}_{1}-\mathrm{C}_{2}$ & 1.394 & $\mathrm{C}_{1}-\mathrm{C}_{2}-\mathrm{C}_{3}$ & 120.2 \\
\hline $\mathrm{C}_{2}-\mathrm{C}_{3}$ & 1.390 & $\mathrm{C}_{2}-\mathrm{C}_{3}-\mathrm{C}_{4}$ & 119.9 \\
\hline $\mathrm{C}_{3}-\mathrm{C}_{4}$ & 1.405 & $\mathrm{C}_{3}-\mathrm{C}_{4}-\mathrm{C}_{5}$ & 119.5 \\
\hline $\mathrm{C}_{4}-\mathrm{C}_{5}$ & 1.406 & $\mathrm{C}_{4}-\mathrm{C}_{5}-\mathrm{C}_{6}$ & 120.3 \\
\hline $\mathrm{C}_{5}-\mathrm{C}_{6}$ & 1.386 & $\mathrm{C}_{5}-\mathrm{C}_{6}-\mathrm{C}_{1}$ & 119.9 \\
\hline $\mathrm{C}_{6}-\mathrm{C}_{1}$ & 1.396 & $\mathrm{C}_{6}-\mathrm{C}_{1}-\mathrm{C}_{2}$ & 120.2 \\
\hline $\mathrm{C}_{7}-\mathrm{C}_{8}$ & 1.558 & $\mathrm{C}_{4}-\mathrm{C}_{7}-\mathrm{C}_{8}$ & 118.9 \\
\hline $\mathrm{C}_{4}-\mathrm{C}_{7}$ & 1.476 & $\mathrm{C}_{3}-\mathrm{C}_{4}-\mathrm{C}_{7}$ & 123.3 \\
\hline $\mathrm{C}_{7}-\mathrm{O}$ & 1.214 & $\mathrm{C}_{4}-\mathrm{C}_{7}-\mathrm{O}$ & 123.3 \\
\hline $\mathrm{C}_{8}-\mathrm{F}$ & $1.349 ; 1.349 ; 1.332$ & $\mathrm{C}_{7}-\mathrm{C}_{8}-\mathrm{F}$ & $110.8 ; 110.7 ; 111.2$ \\
\hline Ga-O & 2.557 & $\mathrm{C}_{7}-\mathrm{O}-\mathrm{Ga}$ & 151.2 \\
\hline $\mathrm{Ga}-\mathrm{C}_{12}$ & 2.010 & $\mathrm{O}-\mathrm{Ga}-\mathrm{C}_{12}$ & 92.3 \\
\hline Ga-C ${ }_{13}$ & 2.006 & O-Ga-C $C_{13}$ & 99.1 \\
\hline $\mathrm{Ga}-\mathrm{C}_{14}$ & 2.003 & O-Ga-C 14 & 89.7 \\
\hline $\mathrm{C}_{12}-\mathrm{Si}_{15}$ & 1.883 & $\mathrm{Ga}-\mathrm{C}_{12}-\mathrm{Si}_{15}$ & 120.0 \\
\hline $\mathrm{C}_{13}-\mathrm{Si}_{16}$ & 1.881 & $\mathrm{Ga}-\mathrm{C}_{13}-\mathrm{Si}_{16}$ & 123.2 \\
\hline $\begin{array}{l}\mathrm{C}_{14}-\mathrm{Si}_{17} \\
\mathrm{Si}_{15}-\mathrm{C}_{\mathrm{Me}}\end{array}$ & $\begin{array}{c}1.883 \\
1.896 ; 1.897 ; 1.893\end{array}$ & $\begin{array}{l}\mathrm{Ga}-\mathrm{C}_{14}-\mathrm{Si}_{17} \\
\mathrm{C}_{12}-\mathrm{Si}_{15}-\mathrm{C}_{\mathrm{Me}}\end{array}$ & $\begin{array}{c}119.5 \\
110.4 ; 110.1 ; 111.6\end{array}$ \\
\hline $\mathrm{Si}_{16}-\mathrm{C}_{\mathrm{Me}}$ & $1.896 ; 1.896 ; 1.894$ & $\mathrm{C}_{13}-\mathrm{Si}_{16}-\mathrm{C}_{\mathrm{Me}}$ & $111.8 ; 109.7 ; 111.0$ \\
\hline $\mathrm{Si}_{17}-\mathrm{C}_{\mathrm{Me}}$ & $1.894 ; 1.894 ; 1.896$ & $\mathrm{C}_{14}-\mathrm{Si}_{17}-\mathrm{C}_{\mathrm{Me}}$ & $110.1 ; 110.2 ; 111.2$ \\
\hline
\end{tabular}

12. Optimized geometry of $I^{t} \mathrm{Bu}+$ trifluoroacetophenone 


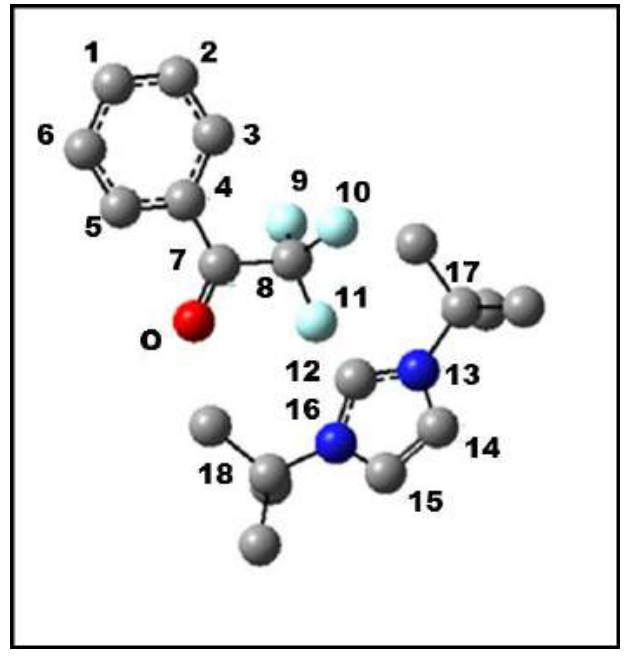

\begin{tabular}{|c|c|c|c|}
\hline \multicolumn{2}{|c|}{ Principal bond lengths $(\AA)$} & \multicolumn{2}{|c|}{ Principal bond angles $\left({ }^{\circ}\right)$} \\
\hline $\mathrm{C}_{1}-\mathrm{C}_{2}$ & 1.393 & $C_{1}-C_{2}-C_{3}$ & 120.2 \\
\hline $\mathrm{C}_{2}-\mathrm{C}_{3}$ & 1.391 & $\mathrm{C}_{2}-\mathrm{C}_{3}-\mathrm{C}_{4}$ & 120.0 \\
\hline $\mathrm{C}_{3}-\mathrm{C}_{4}$ & 1.403 & $\mathrm{C}_{3}-\mathrm{C}_{4}-\mathrm{C}_{5}$ & 119.4 \\
\hline $\mathrm{C}_{4}-\mathrm{C}_{5}$ & 1.404 & $\mathrm{C}_{4}-\mathrm{C}_{5}-\mathrm{C}_{6}$ & 120.4 \\
\hline $\mathrm{C}_{5}-\mathrm{C}_{6}$ & 1.387 & $\mathrm{C}_{5}-\mathrm{C}_{6}-\mathrm{C}_{1}$ & 119.9 \\
\hline $\mathrm{C}_{6}-\mathrm{C}_{1}$ & 1.396 & $\mathrm{C}_{6}-\mathrm{C}_{1}-\mathrm{C}_{2}$ & 120.1 \\
\hline $\mathrm{C}_{4}-\mathrm{C}_{7}$ & 1.486 & $\mathrm{C}_{3}-\mathrm{C}_{4}-\mathrm{C}_{7}$ & 123.7 \\
\hline $\mathrm{C}_{7}-\mathrm{C}_{8}$ & 1.558 & $\mathrm{C}_{4}-\mathrm{C}_{7}-\mathrm{C}_{8}$ & 118.5 \\
\hline $\mathrm{C}_{7}-\mathrm{O}$ & 1.207 & $\mathrm{C}_{4}-\mathrm{C}_{7}-\mathrm{O}$ & 123.8 \\
\hline $\mathrm{C}_{8}-\mathrm{F}$ & $1.355 ; 1.351 ; 1.329$ & $\mathrm{C}_{7}-\mathrm{C}_{8}-\mathrm{F}$ & $110.2 ; 111.6 ; 111.5$ \\
\hline $\mathrm{C}_{7} \cdots \cdots \mathrm{C}_{12}$ & 4.475 & $\mathrm{C}_{4}-\mathrm{C}_{7}-\mathrm{C}_{12}$ & 119.6 \\
\hline $\mathrm{C}_{12}-\mathrm{N}_{13}$ & 1.365 & $\mathrm{C}_{7}-\mathrm{C}_{12}-\mathrm{N}_{13}$ & 137.7 \\
\hline $\mathrm{N}_{13}-\mathrm{C}_{14}$ & 1.393 & $\mathrm{C}_{12}-\mathrm{N}_{13}-\mathrm{C}_{14}$ & 112.1 \\
\hline $\mathrm{C}_{14}-\mathrm{C}_{15}$ & 1.354 & $\mathrm{~N}_{13}-\mathrm{C}_{14}-\mathrm{C}_{15}$ & 106.3 \\
\hline $\mathrm{C}_{15}-\mathrm{N}_{16}$ & 1.392 & $\mathrm{C}_{14}-\mathrm{C}_{15}-\mathrm{N}_{16}$ & 106.4 \\
\hline $\mathrm{C}_{12}-\mathrm{N}_{16}$ & 1.365 & $\mathrm{C}_{15}-\mathrm{N}_{16}-\mathrm{C}_{12}$ & 112.0 \\
\hline $\mathrm{N}_{13}-\mathrm{C}_{17}$ & 1.493 & $\mathrm{C}_{16}-\mathrm{C}_{12}-\mathrm{N}_{13}$ & 103.2 \\
\hline $\mathrm{N}_{16}-\mathrm{C}_{18}$ & 1.495 & $\mathrm{C}_{12}-\mathrm{N}_{13}-\mathrm{C}_{17}$ & 121.8 \\
\hline & & $\mathrm{C}_{12}-\mathrm{N}_{16}-\mathrm{C}_{18}$ & 121.7 \\
\hline $\mathrm{C}_{17}-\mathrm{C}_{\mathrm{Me}}$ & $1.537 ; 1.538 ; 1.535$ & $\mathrm{~N}_{13}-\mathrm{C}_{17}-\mathrm{C}_{\mathrm{Me}}$ & $108.5 ; 110.2 ; 108.3$ \\
\hline $\mathrm{C}_{18}-\mathrm{C}_{\mathrm{Me}}$ & $1.537 ; 1.538 ; 1.534$ & $\mathrm{~N}_{16}-\mathrm{C}_{18}-\mathrm{C}_{\mathrm{Me}}$ & $108.3 ; 108.4 ; 110.1$ \\
\hline
\end{tabular}

13. Optimized geometry of $a l^{\mathrm{t}} \mathrm{Bu}+$ trifluoroacetophenone 


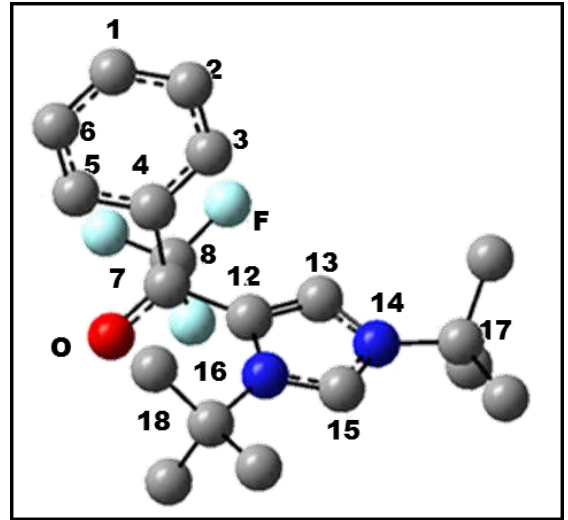

\begin{tabular}{|c|c|c|c|}
\hline \multicolumn{2}{|c|}{ Principal bond lengths $(\stackrel{\AA}{)})$} & \multicolumn{2}{|c|}{ Principal bond angles $\left({ }^{\circ}\right)$} \\
\hline $\mathrm{C}_{1}-\mathrm{C}_{2}$ & 1.393 & $\mathrm{C}_{1}-\mathrm{C}_{2}-\mathrm{C}_{3}$ & 120.1 \\
\hline $\mathrm{C}_{2}-\mathrm{C}_{3}$ & 1.395 & $\mathrm{C}_{2}-\mathrm{C}_{3}-\mathrm{C}_{4}$ & 120.7 \\
\hline $\mathrm{C}_{3}-\mathrm{C}_{4}$ & 1.397 & $\mathrm{C}_{3}-\mathrm{C}_{4}-\mathrm{C}_{5}$ & 118.7 \\
\hline $\mathrm{C}_{4}-\mathrm{C}_{5}$ & 1.396 & $\mathrm{C}_{4}-\mathrm{C}_{5}-\mathrm{C}_{6}$ & 120.8 \\
\hline $\mathrm{C}_{5}-\mathrm{C}_{6}$ & 1.392 & $\mathrm{C}_{5}-\mathrm{C}_{6}-\mathrm{C}_{1}$ & 120.2 \\
\hline $\mathrm{C}_{6}-\mathrm{C}_{1}$ & 1.394 & $\mathrm{C}_{6}-\mathrm{C}_{1}-\mathrm{C}_{2}$ & 119.4 \\
\hline $\mathrm{C}_{7}-\mathrm{C}_{8}$ & 1.581 & $\mathrm{C}_{4}-\mathrm{C}_{7}-\mathrm{C}_{8}$ & 106.2 \\
\hline $\mathrm{C}_{4}-\mathrm{C}_{7}$ & 1.576 & $\mathrm{C}_{3}-\mathrm{C}_{4}-\mathrm{C}_{7}$ & 125.6 \\
\hline $\mathrm{C}_{7}-\mathrm{O}$ & 1.316 & $\mathrm{C}_{4}-\mathrm{C}_{7}-\mathrm{O}$ & 114.0 \\
\hline $\mathrm{C}_{8}-\mathrm{F}$ & $1.384 ; 1.352 ; 1.336$ & $\mathrm{C}_{7}-\mathrm{C}_{8}-\mathrm{F}$ & $115.1 ; 111.8 ; 110.6$ \\
\hline $\mathrm{C}_{7}-\mathrm{C}_{12}$ & 1.576 & $\mathrm{C}_{4}-\mathrm{C}_{7}-\mathrm{C}_{12}$ & 108.8 \\
\hline $\mathrm{C}_{12}-\mathrm{C}_{13}$ & 1.368 & $\mathrm{C}_{7}-\mathrm{C}_{12}-\mathrm{C}_{13}$ & 128.3 \\
\hline $\mathrm{C}_{13}-\mathrm{N}_{14}$ & 1.381 & $\mathrm{C}_{12}-\mathrm{C}_{13}-\mathrm{N}_{14}$ & 109.2 \\
\hline $\mathrm{N}_{14}-\mathrm{C}_{15}$ & 1.334 & $\mathrm{C}_{13}-\mathrm{N}_{14}-\mathrm{C}_{15}$ & 107.1 \\
\hline $\mathrm{C}_{15}-\mathrm{N}_{16}$ & 1.340 & $\mathrm{~N}_{14}-\mathrm{C}_{15}-\mathrm{N}_{16}$ & 110.3 \\
\hline $\mathrm{C}_{12}-\mathrm{N}_{16}$ & 1.409 & $\mathrm{C}_{15}-\mathrm{N}_{16}-\mathrm{C}_{12}$ & 108.1 \\
\hline $\mathrm{N}_{14}-\mathrm{C}_{17}$ & 1.500 & $C_{16}-C_{12}-C_{13}$ & 105.2 \\
\hline $\mathrm{N}_{16}-\mathrm{C}_{18}$ & 1.532 & $\mathrm{C}_{13}-\mathrm{N}_{14}-\mathrm{C}_{17}$ & 125.2 \\
\hline & & $\mathrm{C}_{12}-\mathrm{N}_{16}-\mathrm{C}_{18}$ & 130.7 \\
\hline $\mathrm{C}_{17}-\mathrm{C}_{\mathrm{Me}}$ & $1.537 ; 1.537 ; 1.533$ & $\mathrm{~N}_{14}-\mathrm{C}_{17}-\mathrm{C}_{\mathrm{Me}}$ & $108.1 ; 107.9 ; 109.7$ \\
\hline $\mathrm{C}_{18}-\mathrm{C}_{\mathrm{Me}}$ & $1.538 ; 1.536 ; 1.532$ & $\mathrm{~N}_{16}-\mathrm{C}_{18}-\mathrm{C}_{\mathrm{Me}}$ & $109.3 ; 107.5 ; 108.9$ \\
\hline
\end{tabular}

14. Optimized geometry of $\mathrm{II}_{\mathrm{CF} 3}$ 


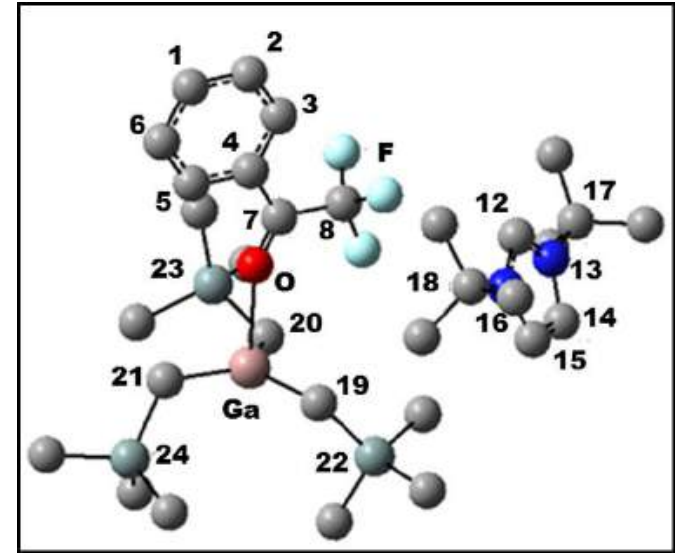

\begin{tabular}{|c|c|c|c|c|c|}
\hline \multicolumn{2}{|c|}{ Principal bond lengths $(\AA)$} & \multicolumn{2}{|c|}{ Principal bond angles $\left({ }^{\circ}\right)$} & \multicolumn{2}{|c|}{ Principal Bond Indices } \\
\hline $\mathrm{C}_{1}-\mathrm{C}_{2}$ & 1.393 & $\mathrm{C}_{1}-\mathrm{C}_{2}-\mathrm{C}_{3}$ & 120.2 & $\mathrm{C}_{1}-\mathrm{C}_{2}$ & 1.43 \\
\hline $\mathrm{C}_{2}-\mathrm{C}_{3}$ & 1.390 & $\mathrm{C}_{2}-\mathrm{C}_{3}-\mathrm{C}_{4}$ & 119.9 & $\mathrm{C}_{2}-\mathrm{C}_{3}$ & 1.45 \\
\hline $\mathrm{C}_{3}-\mathrm{C}_{4}$ & 1.405 & $\mathrm{C}_{3}-\mathrm{C}_{4}-\mathrm{C}_{5}$ & 119.5 & $\mathrm{C}_{3}-\mathrm{C}_{4}$ & 1.35 \\
\hline $\mathrm{C}_{4}-\mathrm{C}_{5}$ & 1.406 & $\mathrm{C}_{4}-\mathrm{C}_{5}-\mathrm{C}_{6}$ & 120.3 & $\mathrm{C}_{4}-\mathrm{C}_{5}$ & 1.34 \\
\hline $\mathrm{C}_{5}-\mathrm{C}_{6}$ & 1.386 & $\mathrm{C}_{5}-\mathrm{C}_{6}-\mathrm{C}_{1}$ & 119.9 & $\mathrm{C}_{5}-\mathrm{C}_{6}$ & 1.47 \\
\hline $\mathrm{C}_{6}-\mathrm{C}_{1}$ & 1.396 & $\mathrm{C}_{6}-\mathrm{C}_{1}-\mathrm{C}_{2}$ & 120.2 & $\mathrm{C}_{6}-\mathrm{C}_{1}$ & 1.41 \\
\hline $\mathrm{C}_{4}-\mathrm{C}_{7}$ & 1.476 & $\mathrm{C}_{3}-\mathrm{C}_{4}-\mathrm{C}_{7}$ & 123.3 & $\mathrm{C}_{4}-\mathrm{C}_{7}$ & 1.08 \\
\hline $\mathrm{C}_{7}-\mathrm{C}_{8}$ & 1.559 & $\mathrm{C}_{4}-\mathrm{C}_{7}-\mathrm{C}_{8}$ & 118.8 & $\mathrm{C}_{7}-\mathrm{C}_{8}$ & 0.91 \\
\hline $\mathrm{C}_{7}-\mathrm{O}$ & 1.214 & $\mathrm{C}_{4}-\mathrm{C}_{7}-\mathrm{O}$ & 123.3 & $\mathrm{C}_{7}-\mathrm{O}$ & 1.22 \\
\hline $\mathrm{C}_{8}-\mathrm{F}$ & $1.350 ; 1.346 ; 1.334$ & $\mathrm{C}_{7}-\mathrm{C}_{8}-\mathrm{F}$ & $110.6 ; 110.9 ; 111.2$ & $\mathrm{C}_{8}-\mathrm{F}$ & $0.87 ; 0.88 ; 0.90$ \\
\hline$C_{7} \cdots \cdot C_{12}$ & 5.708 & $\mathrm{C}_{12}-\mathrm{C}_{13}-\mathrm{N}_{14}$ & 112.2 & $\mathrm{C}_{7} \cdots \cdots \mathrm{C}_{12}$ & 0.0001 \\
\hline $\mathrm{C}_{12}-\mathrm{N}_{13}$ & 1.365 & $\mathrm{C}_{13}-\mathrm{N}_{14}-\mathrm{C}_{15}$ & 106.4 & $\mathrm{C}_{12}-\mathrm{N}_{13}$ & 1.26 \\
\hline $\mathrm{N}_{13}-\mathrm{C}_{14}$ & 1.394 & $\mathrm{~N}_{14}-\mathrm{C}_{15}-\mathrm{N}_{16}$ & 106.3 & $\mathrm{~N}_{13}-\mathrm{C}_{14}$ & 1.10 \\
\hline $\mathrm{C}_{14}-\mathrm{C}_{15}$ & 1.352 & $\mathrm{C}_{15}-\mathrm{N}_{16}-\mathrm{C}_{12}$ & 112.2 & $\mathrm{C}_{14}-\mathrm{C}_{15}$ & 1.67 \\
\hline $\mathrm{C}_{15}-\mathrm{N}_{16}$ & 1.394 & $\mathrm{~N}_{16}-\mathrm{C}_{12}-\mathrm{N}_{13}$ & 103.0 & $\mathrm{C}_{15}-\mathrm{N}_{16}$ & 1.10 \\
\hline $\mathrm{N}_{16}-\mathrm{C}_{12}$ & 1.366 & $\mathrm{C}_{12}-\mathrm{N}_{13}-\mathrm{C}_{17}$ & 124.6 & $N_{16}-C_{12}$ & 1.26 \\
\hline $\mathrm{N}_{13}-\mathrm{C}_{17}$ & 1.489 & $\mathrm{C}_{12}-\mathrm{N}_{16}-\mathrm{C}_{18}$ & 124.2 & $\mathrm{~N}_{13}-\mathrm{C}_{17}$ & 0.90 \\
\hline $\mathrm{N}_{16}-\mathrm{C}_{18}$ & 1.489 & $\mathrm{~N}_{13}-\mathrm{C}_{17}-\mathrm{C}_{\mathrm{Me}}$ & $108.7 ; 109.7 ; 108.7$ & $\mathrm{~N}_{16}-\mathrm{C}_{18}$ & 0.90 \\
\hline $\mathrm{C}_{17}-\mathrm{C}_{\mathrm{Me}}$ & $1.533 ; 1.540 ; 1.540$ & $\mathrm{~N}_{16}-\mathrm{C}_{18}-\mathrm{C}_{\mathrm{Me}}$ & $108.6 ; 109.5 ; 109.1$ & $\mathrm{C}_{17}-\mathrm{C}_{\mathrm{Me}}$ & $0.99 ; 1.00 ; 0.99$ \\
\hline $\mathrm{C}_{18}-\mathrm{C}_{\mathrm{Me}}$ & $1.540 ; 1.539 ; 1.533$ & $\mathrm{C}_{7}-\mathrm{O}-\mathrm{Ga}$ & 153.5 & $\mathrm{C}_{18}-\mathrm{C}_{\mathrm{Me}}$ & $1.00 ; 0.99 ; 0.99$ \\
\hline O-Ga & 2.562 & O-Ga-C 19 & 92.7 & O-Ga & 0.12 \\
\hline Ga-C 19 & 2.008 & O-Ga-C 20 & 98.3 & Ga-C 19 & 0.61 \\
\hline $\mathrm{Ga}-\mathrm{C}_{20}$ & 2.007 & O-Ga-C ${ }_{21}$ & 90.1 & $\mathrm{Ga}-\mathrm{C}_{20}$ & 0.60 \\
\hline $\mathrm{Ga}-\mathrm{C}_{21}$ & 2.003 & $\mathrm{Ga}-\mathrm{C}_{19}-\mathrm{Si}_{22}$ & 120.2 & Ga-C $C_{21}$ & 0.60 \\
\hline $\mathrm{C}_{19}-\mathrm{Si}_{22}$ & 1.882 & $\mathrm{Ga}-\mathrm{C}_{20}-\mathrm{Si}_{23}$ & 123.4 & $\mathrm{C}_{19}-\mathrm{Si}_{22}$ & 0.82 \\
\hline $\mathrm{C}_{20}-\mathrm{Si}_{23}$ & 1.880 & $\mathrm{Ga}-\mathrm{C}_{21}-\mathrm{Si}_{24}$ & 119.6 & $\mathrm{C}_{20}-\mathrm{Si}_{23}$ & 0.82 \\
\hline $\mathrm{C}_{21}-\mathrm{Si}_{24}$ & 1.883 & $\mathrm{C}_{19}-\mathrm{Si}_{22}-\mathrm{C}_{\mathrm{Me}}$ & $110.7 ; 111.6 ; 110.0$ & $\mathrm{C}_{21}-\mathrm{Si}_{24}$ & 0.82 \\
\hline
\end{tabular}




\begin{tabular}{llllll}
\hline $\mathbf{S i}_{22}-\mathbf{C}_{\mathrm{Me}}$ & $1.893 ; 1.897 ; 1.896$ & $\mathbf{C}_{20}-\mathbf{S i}_{23}-\mathbf{C}_{\mathrm{Me}}$ & $111.7 ; 109.7 ; 111.1$ & $\mathbf{S i}_{22}-\mathbf{C}_{\mathrm{Me}}$ & $0.82 ; 0.82 ; 0.82$ \\
$\mathbf{S i}_{23}-\mathbf{C}_{\mathrm{Me}}$ & $1.896 ; 1.896 ; 1.894$ & $\mathbf{C}_{21}-\mathbf{S i}_{24}-\mathbf{C}_{\mathrm{Me}}$ & $110.3 ; 110.1 ; 111.4$ & $\mathbf{S i}_{23}-\mathbf{C}_{\mathrm{Me}}$ & $0.82 ; 0.82 ; 0.82$ \\
$\mathbf{S i}_{24}-\mathbf{C}_{\mathrm{Me}}$ & $1.894 ; 1.894 ; 1.896$ & & & $\mathrm{Si}_{24}-\mathbf{C}_{\mathrm{Me}}$ & $0.82 ; 0.82 ; 0.82$ \\
\hline
\end{tabular}

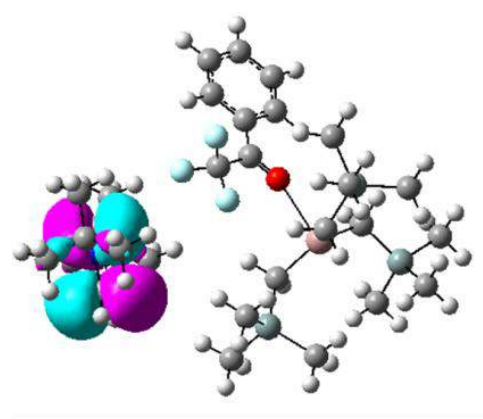

HOMO-1

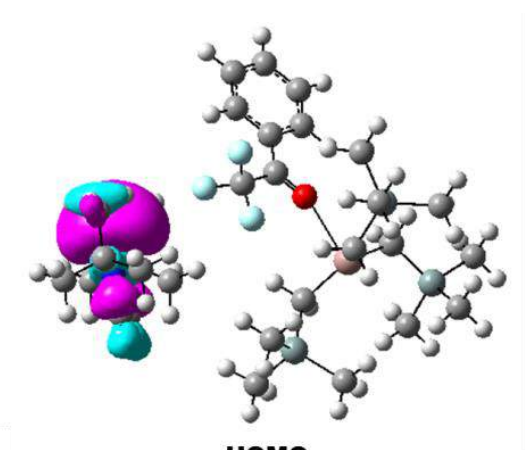

HOMO

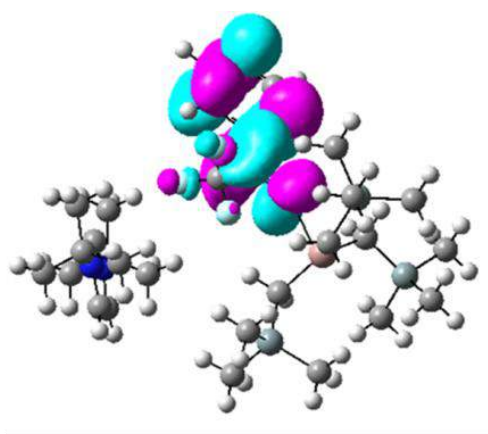

LUMO

Figure S22: Representation of molecular orbitals HOMO-1, HOMO and LUMO of $\mathbf{I I}_{\mathbf{C F 3}}$.

15. Optimized geometry of $\mathrm{III}_{\mathrm{CF} 3}$

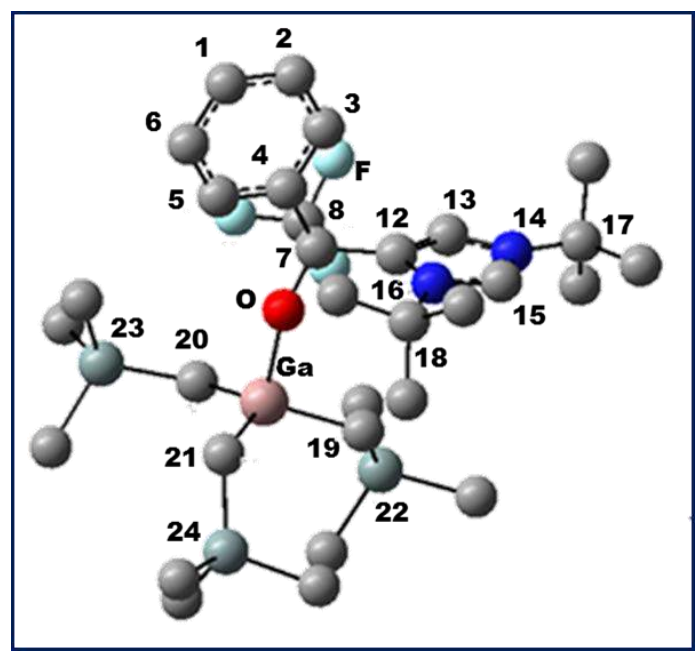

\begin{tabular}{|c|c|c|c|c|c|}
\hline \multicolumn{2}{|c|}{ Principal bond lengths $(\AA)$} & \multicolumn{2}{|c|}{ Principal bond angles $\left({ }^{\circ}\right)$} & \multicolumn{2}{|c|}{ Principal Bond Indices } \\
\hline $\mathrm{C}_{1}-\mathrm{C}_{2}$ & 1.393 & $\mathrm{C}_{1}-\mathrm{C}_{2}-\mathrm{C}_{3}$ & 120.1 & $\mathrm{C}_{1}-\mathrm{C}_{2}$ & 1.44 \\
\hline $\mathrm{C}_{2}-\mathrm{C}_{3}$ & 1.394 & $\mathrm{C}_{2}-\mathrm{C}_{3}-\mathrm{C}_{4}$ & 120.6 & $\mathrm{C}_{2}-\mathrm{C}_{3}$ & 1.43 \\
\hline $\mathrm{C}_{3}-\mathrm{C}_{4}$ & 1.397 & $\mathrm{C}_{3}-\mathrm{C}_{4}-\mathrm{C}_{5}$ & 119.0 & $\mathrm{C}_{3}-\mathrm{C}_{4}$ & 1.41 \\
\hline $\mathrm{C}_{4}-\mathrm{C}_{5}$ & 1.395 & $\mathrm{C}_{4}-\mathrm{C}_{5}-\mathrm{C}_{6}$ & 120.5 & $\mathrm{C}_{4}-\mathrm{C}_{5}$ & 1.40 \\
\hline $\mathrm{C}_{5}-\mathrm{C}_{6}$ & 1.392 & $\mathrm{C}_{5}-\mathrm{C}_{6}-\mathrm{C}_{1}$ & 120.3 & $\mathrm{C}_{5}-\mathrm{C}_{6}$ & 1.43 \\
\hline $\mathrm{C}_{6}-\mathrm{C}_{1}$ & 1.394 & $\mathrm{C}_{6}-\mathrm{C}_{1}-\mathrm{C}_{2}$ & 119.5 & $\mathrm{C}_{6}-\mathrm{C}_{1}$ & 1.43 \\
\hline $\mathrm{C}_{4}-\mathrm{C}_{7}$ & 1.559 & $\mathrm{C}_{3}-\mathrm{C}_{4}-\mathrm{C}_{7}$ & 122.8 & $\mathrm{C}_{4}-\mathrm{C}_{7}$ & 0.94 \\
\hline $\mathrm{C}_{7}-\mathrm{C}_{8}$ & 1.576 & $\mathrm{C}_{4}-\mathrm{C}_{7}-\mathrm{C}_{8}$ & 104.5 & $\mathrm{C}_{7}-\mathrm{C}_{8}$ & 0.90 \\
\hline $\mathrm{C}_{7}-\mathrm{O}$ & 1.355 & $\mathrm{C}_{4}-\mathrm{C}_{7}-\mathrm{O}$ & 112.6 & $\mathrm{C}_{7}-\mathrm{O}$ & 1.04 \\
\hline
\end{tabular}




\begin{tabular}{|c|c|c|c|c|c|}
\hline$C_{8}-F$ & $1.367 ; 1.353 ; 1.335$ & $\mathrm{C}_{7}-\mathrm{C}_{8}-\mathrm{F}$ & $113.4 ; 112.5 ; 110.6$ & $\mathrm{C}_{8}-\mathrm{F}$ & $0.90 ; 0.86 ; 0.95$ \\
\hline $\mathrm{C}_{7}-\mathrm{C}_{12}$ & 1.552 & $C_{4}-C_{7}-C_{12}$ & 110.3 & $\mathrm{C}_{7}-\mathrm{C}_{12}$ & 0.95 \\
\hline $\mathrm{C}_{12}-\mathrm{C}_{13}$ & 1.367 & $\mathrm{C}_{7}-\mathrm{C}_{12}-\mathrm{C}_{13}$ & 126.9 & $\mathrm{C}_{12}-\mathrm{C}_{13}$ & 1.59 \\
\hline $\mathrm{C}_{13}-\mathrm{N}_{14}$ & 1.378 & $\mathrm{C}_{12}-\mathrm{C}_{13}-\mathrm{N}_{14}$ & 109.2 & $\mathrm{C}_{13}-\mathrm{N}_{14}$ & 1.13 \\
\hline $\mathrm{N}_{14}-\mathrm{C}_{15}$ & 1.311 & $\mathrm{C}_{13}-\mathrm{N}_{14}-\mathrm{C}_{15}$ & 107.1 & $\mathrm{~N}_{14}-\mathrm{C}_{15}$ & 1.31 \\
\hline $\mathrm{C}_{15}-\mathrm{N}_{16}$ & 1.340 & $\mathrm{~N}_{14}-\mathrm{C}_{15}-\mathrm{N}_{16}$ & 110.7 & $\mathrm{C}_{15}-\mathrm{N}_{16}$ & 1.33 \\
\hline$N_{16}-C_{12}$ & 1.411 & $\mathrm{C}_{15}-\mathrm{N}_{16}-\mathrm{C}_{12}$ & 107.5 & $N_{16}-C_{12}$ & 1.13 \\
\hline $\mathrm{N}_{14}-\mathrm{C}_{17}$ & 1.504 & $\mathrm{~N}_{16}-\mathrm{C}_{12}-\mathrm{C}_{13}$ & 105.5 & $\mathrm{~N}_{14}-\mathrm{C}_{17}$ & 0.87 \\
\hline $\mathrm{N}_{16}-\mathrm{C}_{18}$ & 1.542 & $\mathrm{C}_{13}-\mathrm{N}_{14}-\mathrm{C}_{17}$ & 125.2 & $\mathrm{~N}_{16}-\mathrm{C}_{18}$ & 0.84 \\
\hline $\mathrm{C}_{17}-\mathrm{C}_{\mathrm{Me}}$ & $1.532 ; 1.537 ; 1.537$ & $\mathrm{C}_{12}-\mathrm{N}_{16}-\mathrm{C}_{18}$ & 133.8 & $\mathrm{C}_{17}-\mathrm{C}_{\mathrm{Me}}$ & $1.00 ; 1.00 ; 1.01$ \\
\hline $\mathrm{C}_{18}-\mathrm{C}_{\mathrm{Me}}$ & $1.541 ; 1.533 ; 1.527$ & $\mathrm{~N}_{14}-\mathrm{C}_{17}-\mathrm{C}_{\mathrm{Me}}$ & $107.9 ; 107.8 ; 109.4$ & $\mathrm{C}_{18}-\mathrm{C}_{\mathrm{Me}}$ & $1.00 ; 1.00 ; 1.01$ \\
\hline & & $\mathrm{N}_{16}-\mathrm{C}_{18}-\mathrm{C}_{\mathrm{Me}}$ & $108.0 ; 106.8 ; 111.9$ & & \\
\hline O-Ga & 2.061 & $\mathrm{C}_{7}-\mathrm{O}-\mathbf{G a}$ & 139.3 & O-Ga & 0.32 \\
\hline Ga-C 19 & 2.058 & O-Ga-C 19 & 101.9 & Ga-C 19 & 0.55 \\
\hline Ga- $C_{20}$ & 2.031 & $\mathrm{O}-\mathrm{Ga}-\mathrm{C}_{20}$ & 108.8 & $\mathrm{Ga}-\mathrm{C}_{20}$ & 0.58 \\
\hline Ga-C ${ }_{21}$ & 2.039 & O-Ga-C $\mathrm{C}_{21}$ & 95.5 & $\mathrm{Ga}-\mathrm{C}_{21}$ & 0.57 \\
\hline $\mathrm{C}_{19}-\mathrm{Si}_{22}$ & 1.873 & $\mathrm{Ga}-\mathrm{C}_{19}-\mathrm{Si}_{22}$ & 122.1 & $\mathrm{C}_{19}-\mathrm{Si}_{22}$ & 0.84 \\
\hline $\mathrm{C}_{20}-\mathrm{Si}_{23}$ & 1.875 & $\mathrm{Ga}-\mathrm{C}_{20}-\mathrm{Si}_{23}$ & 124.2 & $\mathrm{C}_{20}-\mathrm{Si}_{23}$ & 0.83 \\
\hline $\mathrm{C}_{21}-\mathrm{Si}_{24}$ & 1.874 & Ga-C ${ }_{21}-\mathrm{Si}_{24}$ & 123.7 & $\mathrm{C}_{21}-\mathrm{Si}_{24}$ & 0.84 \\
\hline $\mathrm{Si}_{22}-\mathrm{C}_{\mathrm{Me}}$ & $1.895 ; 1.908 ; 1.895$ & $\mathrm{C}_{19}-\mathrm{Si}_{22}-\mathrm{C}_{\mathrm{Me}}$ & $111.8 ; 110.2 ; 112.5$ & $\mathrm{Si}_{22}-\mathrm{C}_{\mathrm{Me}}$ & $0.79 ; 0.82 ; 0.81$ \\
\hline $\mathrm{Si}_{23}-\mathrm{C}_{\mathrm{Me}}$ & $1.901 ; 1.896 ; 1.898$ & $\mathrm{C}_{20}-\mathrm{Si}_{23}-\mathrm{C}_{\mathrm{Me}}$ & $111.2 ; 113.7 ; 110.0$ & $\mathrm{Si}_{23}-\mathrm{C}_{\mathrm{Me}}$ & $0.81 ; 0.81 ; 0.81$ \\
\hline $\mathrm{Si}_{24}-\mathrm{C}_{\mathrm{Me}}$ & $1.903 ; 1.894 ; 1.901$ & $\mathrm{C}_{21}-\mathrm{Si}_{24}-\mathrm{C}_{\mathrm{Me}}$ & $111.7 ; 112.7 ; 110.4$ & $\mathrm{Si}_{24}-\mathrm{C}_{\mathrm{Me}}$ & $0.80 ; 0.81 ; 0.82$ \\
\hline
\end{tabular}

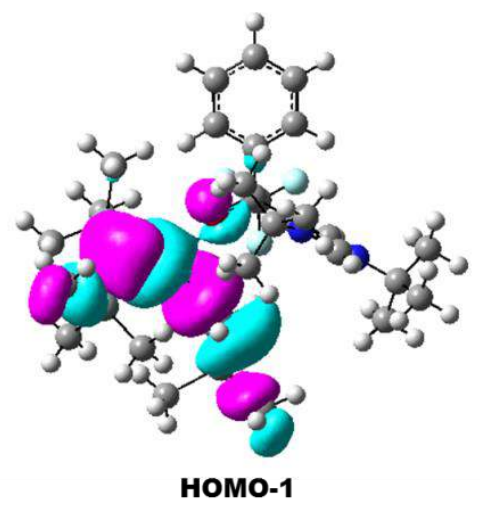

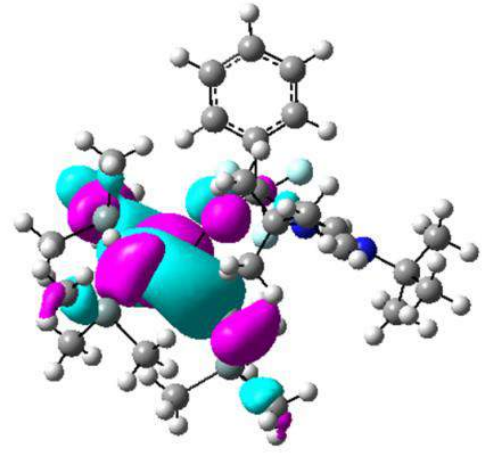

номо

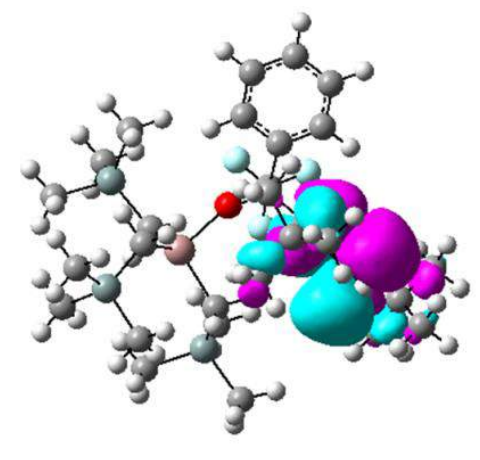

LUMO

Figure S23: Representation of molecular orbitals HOMO-1, HOMO and LUMO of III $_{\mathbf{C F 3}}$. 


\section{NMR spectra of products}

i

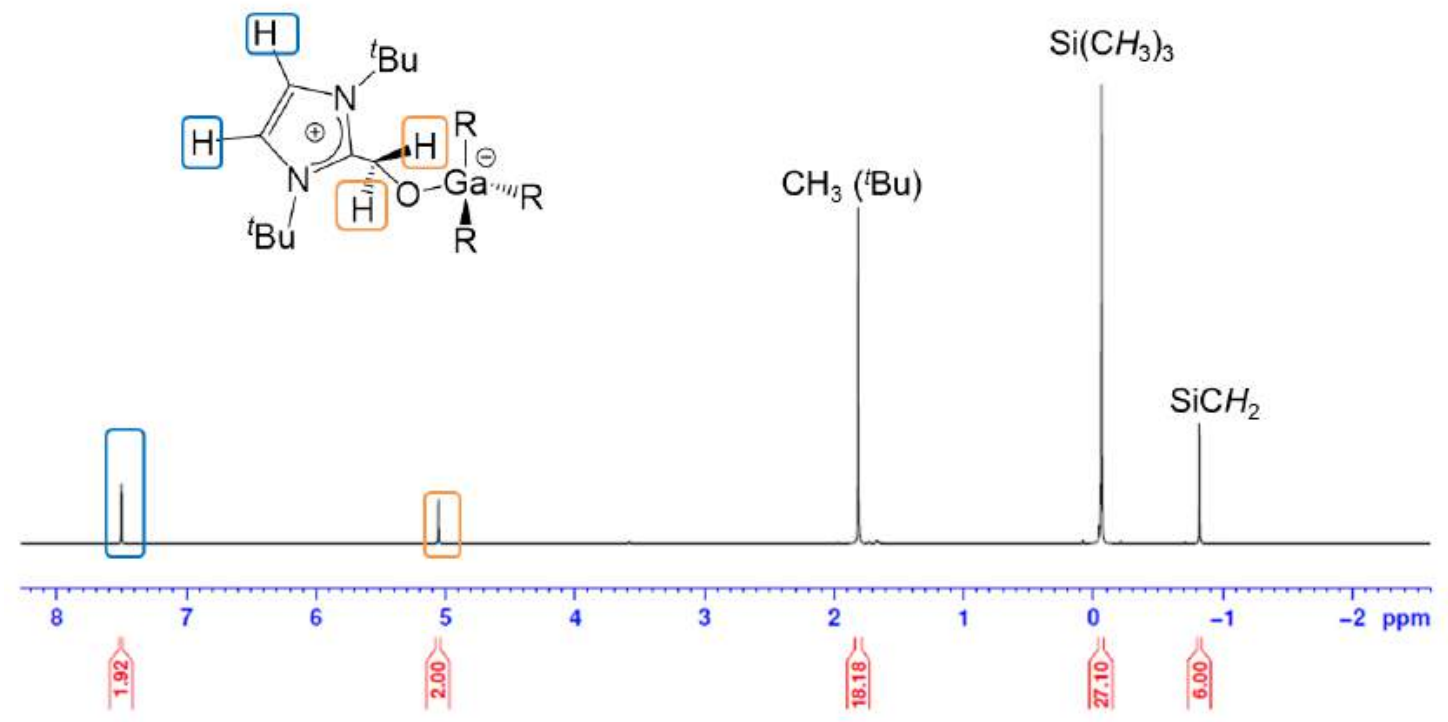

Figure S24: ${ }^{1} \mathrm{H}$ NMR spectrum of $\mathbf{1}$ in $\mathrm{d}_{8}$-THF.

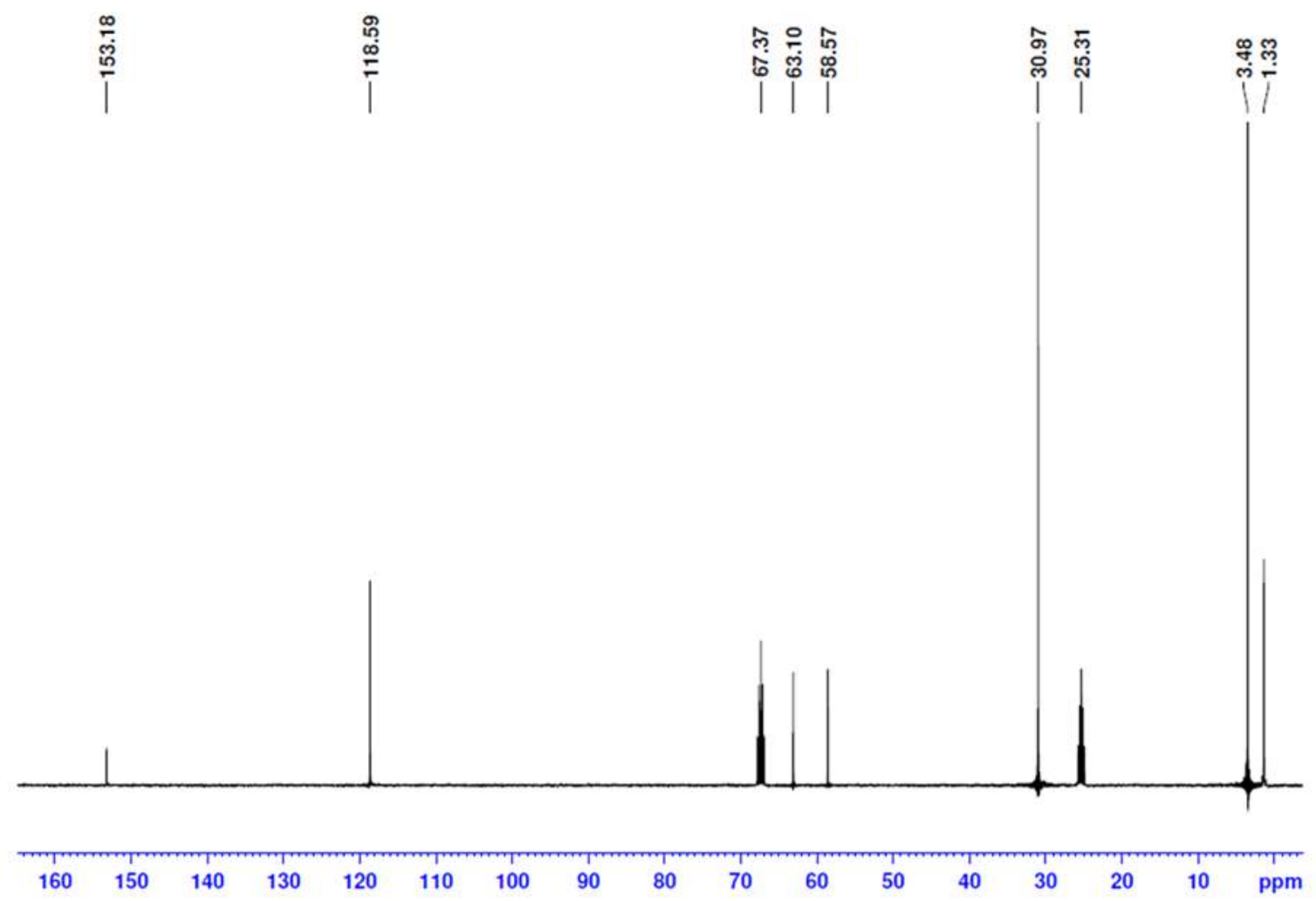

Figure S25: ${ }^{13} \mathrm{C}$ NMR spectrum of $\mathbf{1}$ in $\mathrm{d}_{8}$-THF. 


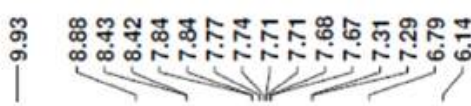<smiles></smiles>

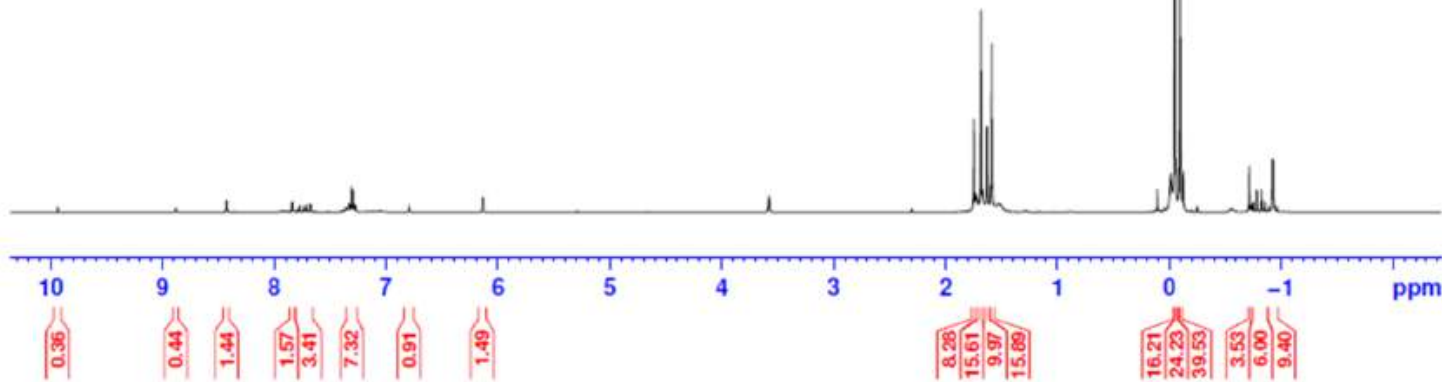

Figure S26: ${ }^{1} \mathrm{H}$ NMR spectrum of $\mathbf{2}$ in $\mathrm{d}_{8}$-THF (presence of $\mathbf{3}$ and free components observed, see further spectra).
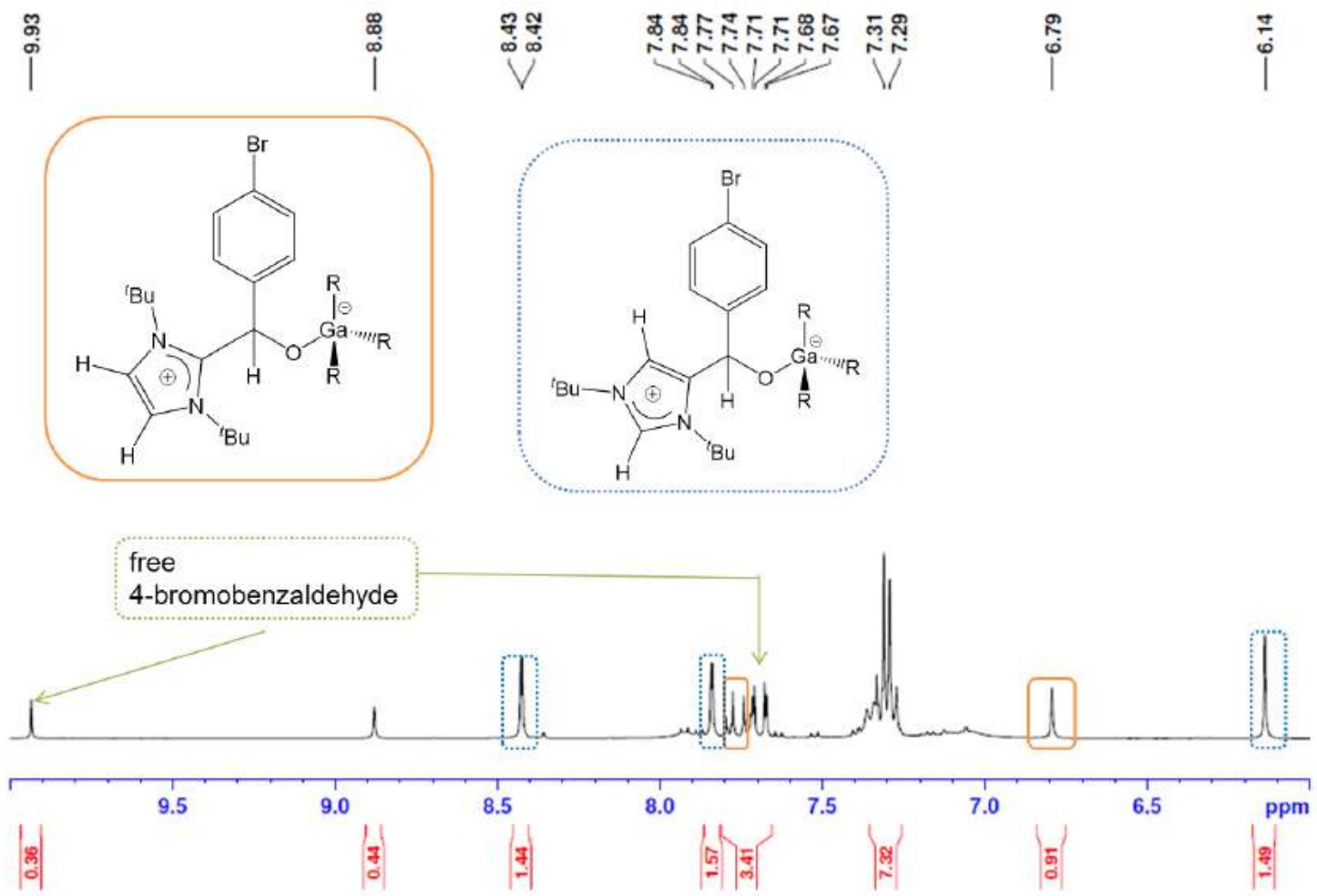

Figure S27: Aromatic region of ${ }^{1} \mathrm{H}$ NMR spectrum of 2 in $\mathrm{d}_{8}$-THF. 


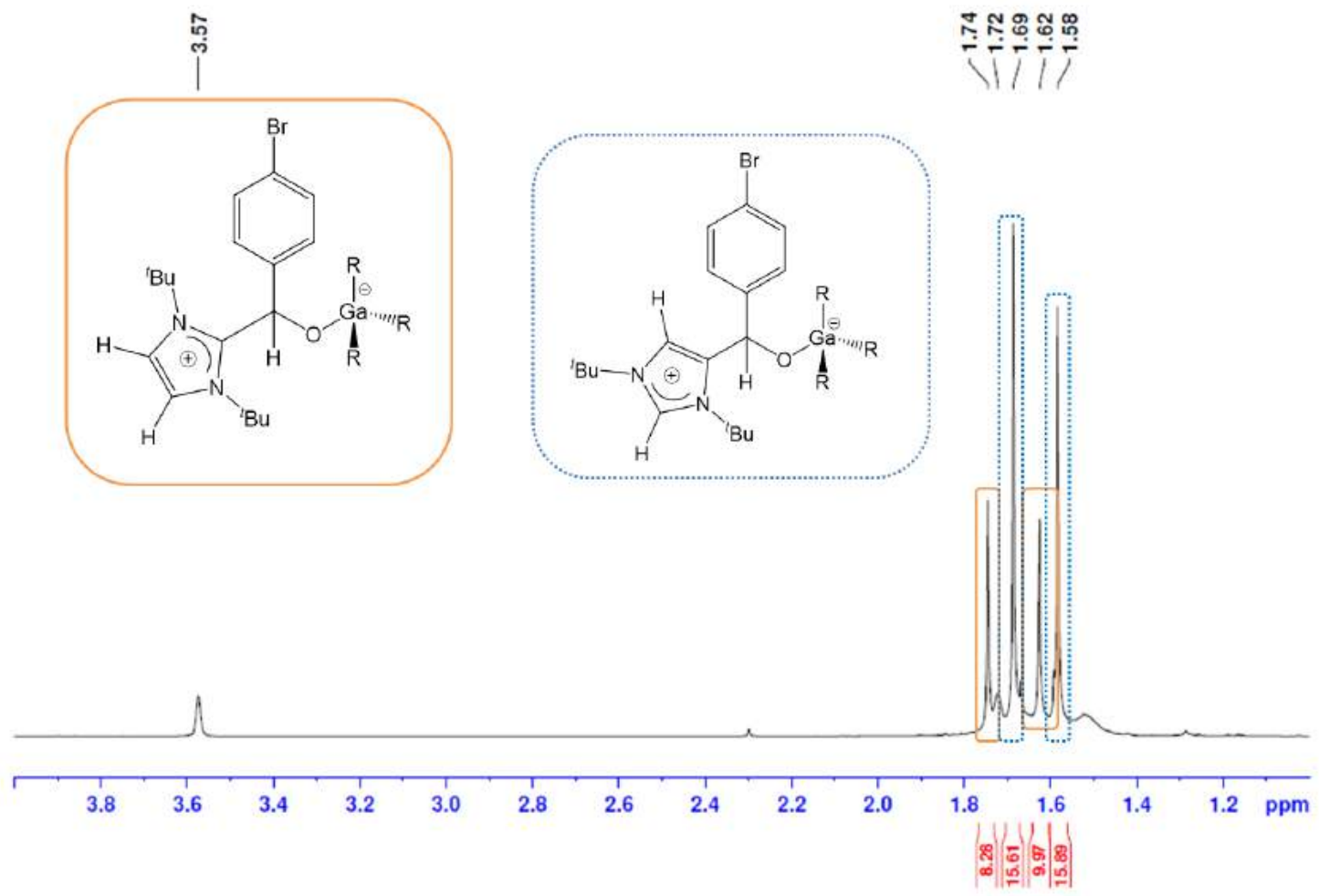

Figure S28: High field region of ${ }^{1} \mathrm{H}$ NMR spectrum of 2 in $\mathrm{d}_{8}$-THF.

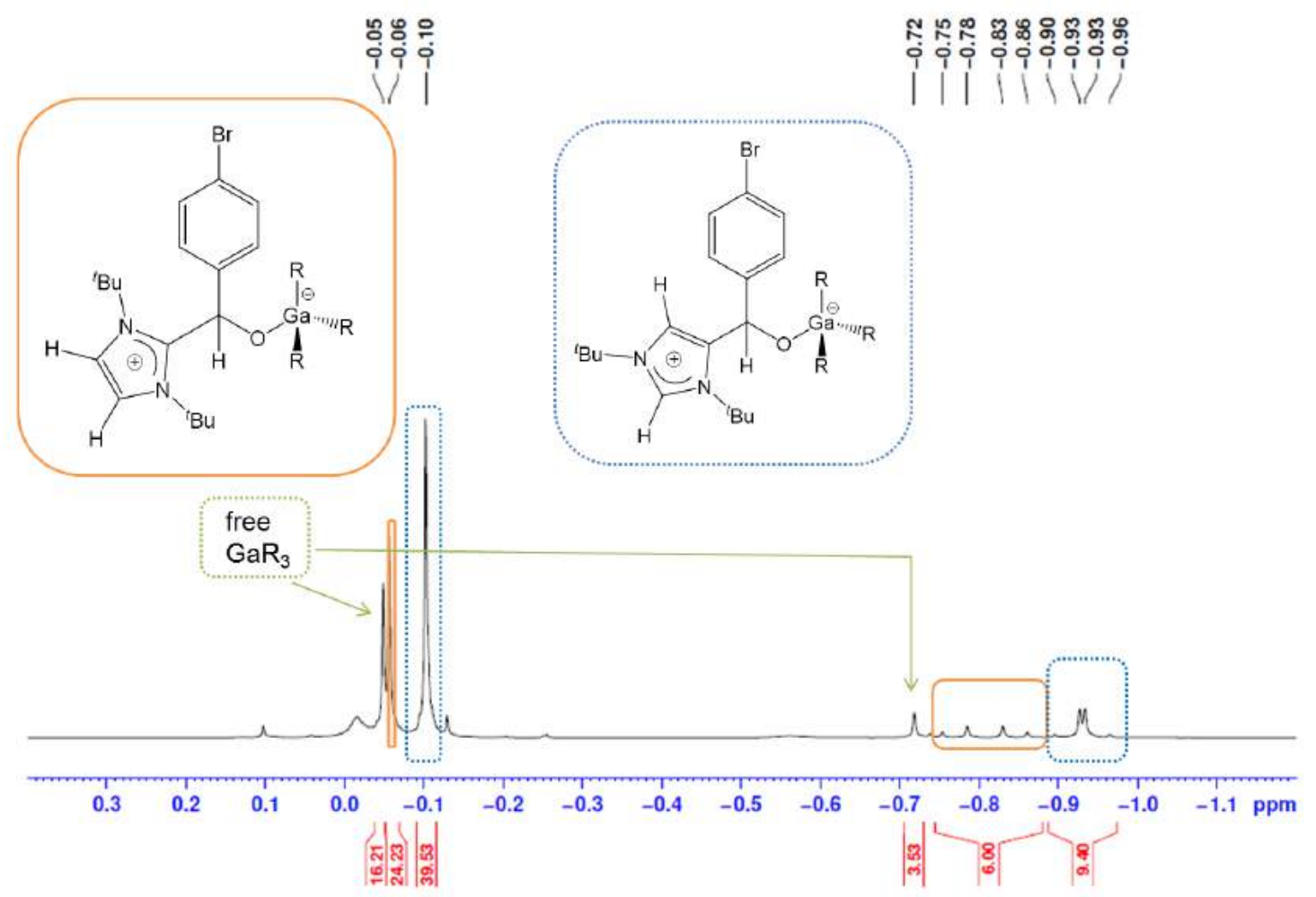

Figure S29: High field region of ${ }^{1} \mathrm{H}$ NMR spectrum of 2 in $\mathrm{d}_{8}$-THF. 


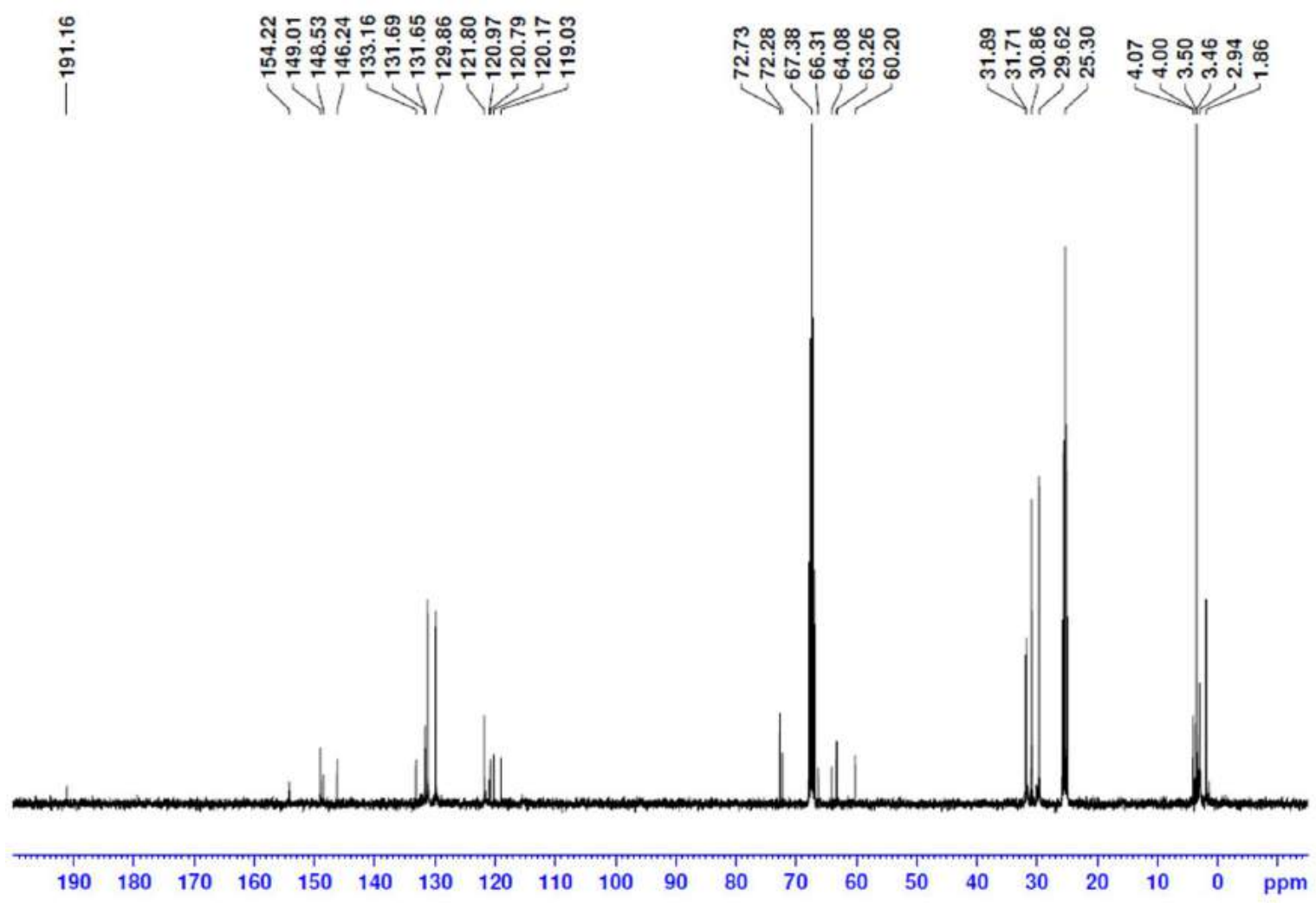

Figure S30: ${ }^{13} \mathrm{C}$ NMR spectrum of $\mathbf{2}$ in $\mathrm{d}_{8}$-THF.

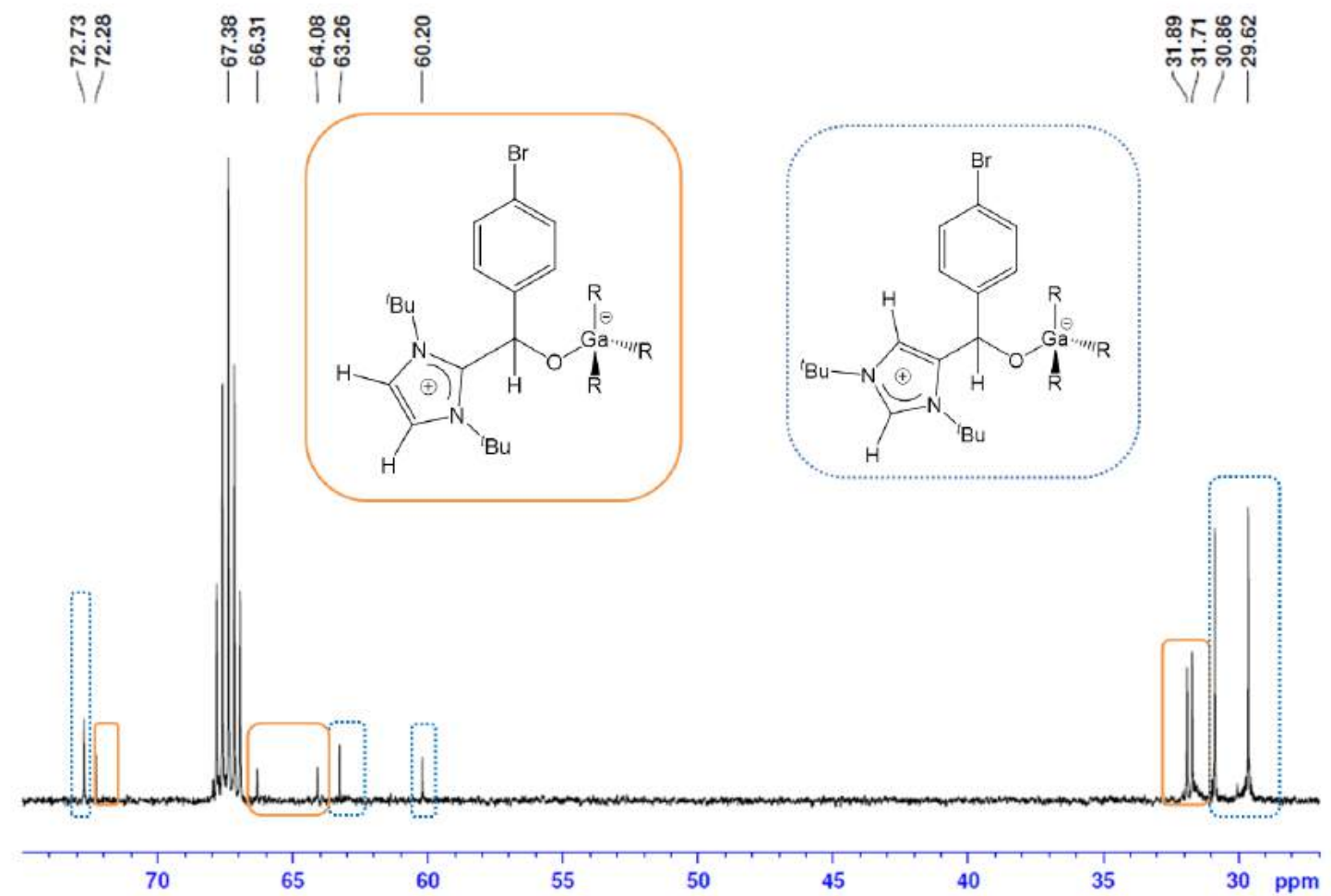

Figure S31: High field region of ${ }^{13} \mathrm{C}$ NMR of 2 in $\mathrm{d}_{8}$-THF. 


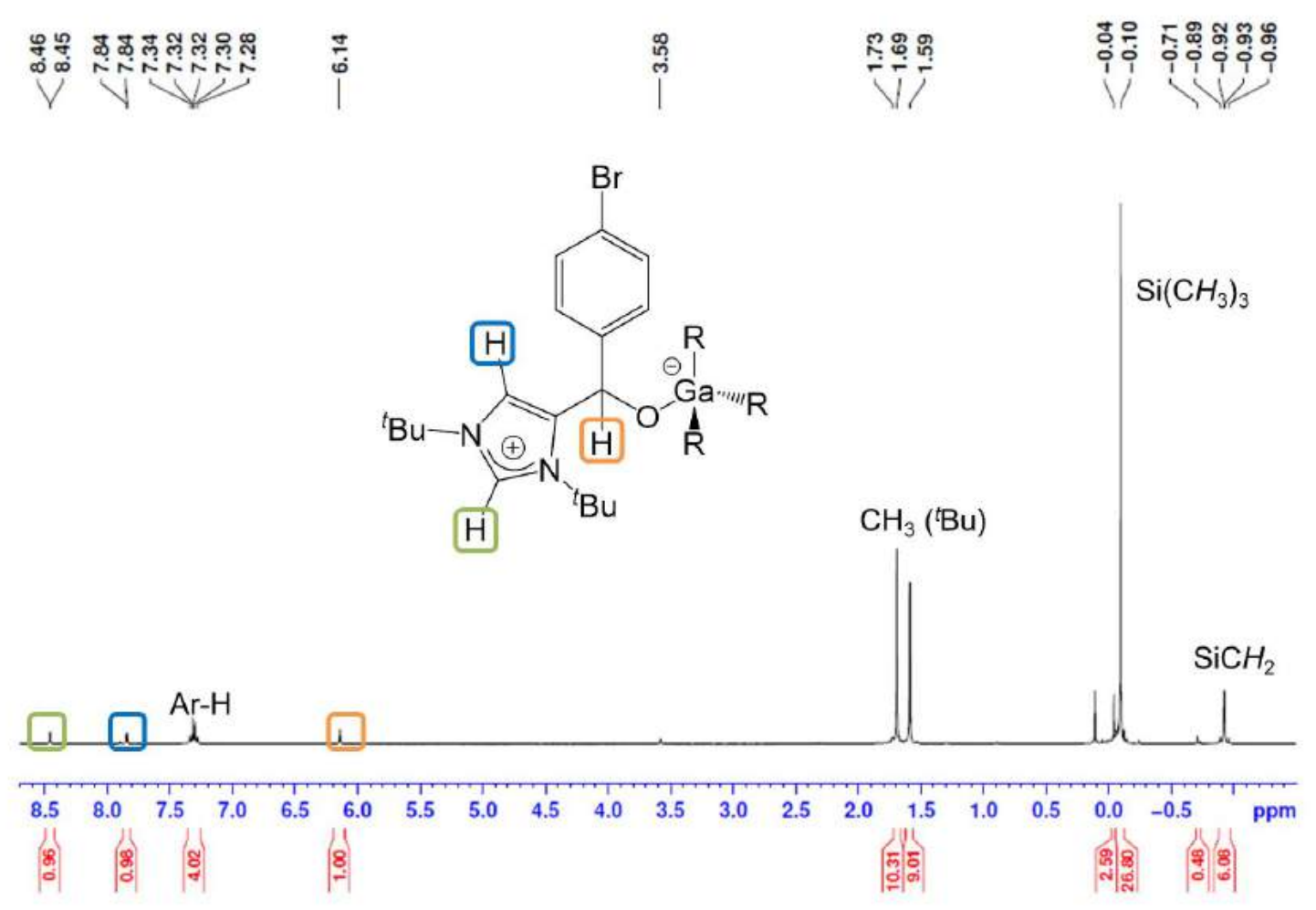

Figure S32: ${ }^{1} \mathrm{H}$ NMR spectrum of 3 in $\mathrm{d}_{8}$-THF.

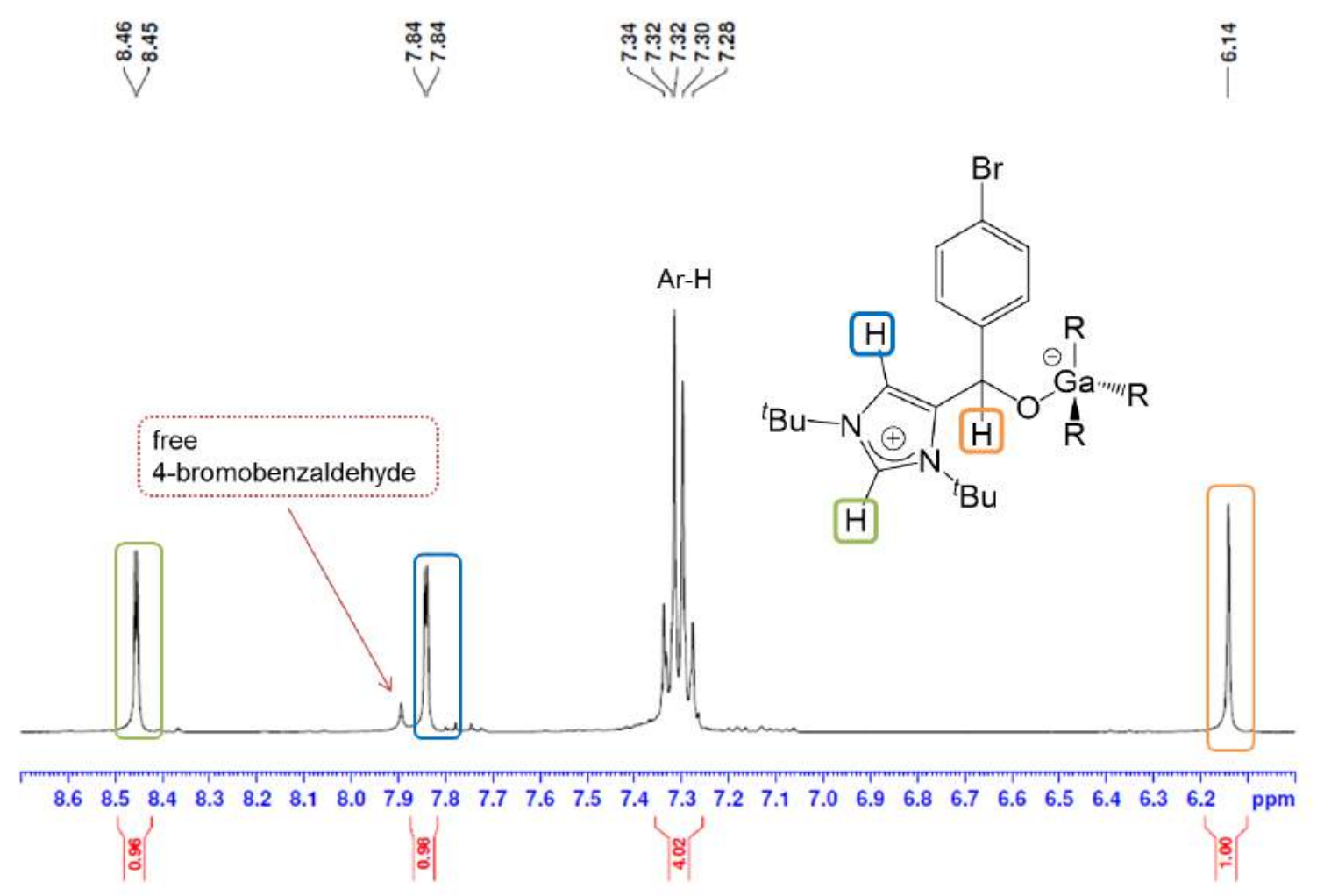

Figure S33: Aromatic region of ${ }^{1} \mathrm{H}$ NMR spectrum of $\mathbf{3}$ in $\mathrm{d}_{8}$-THF. 


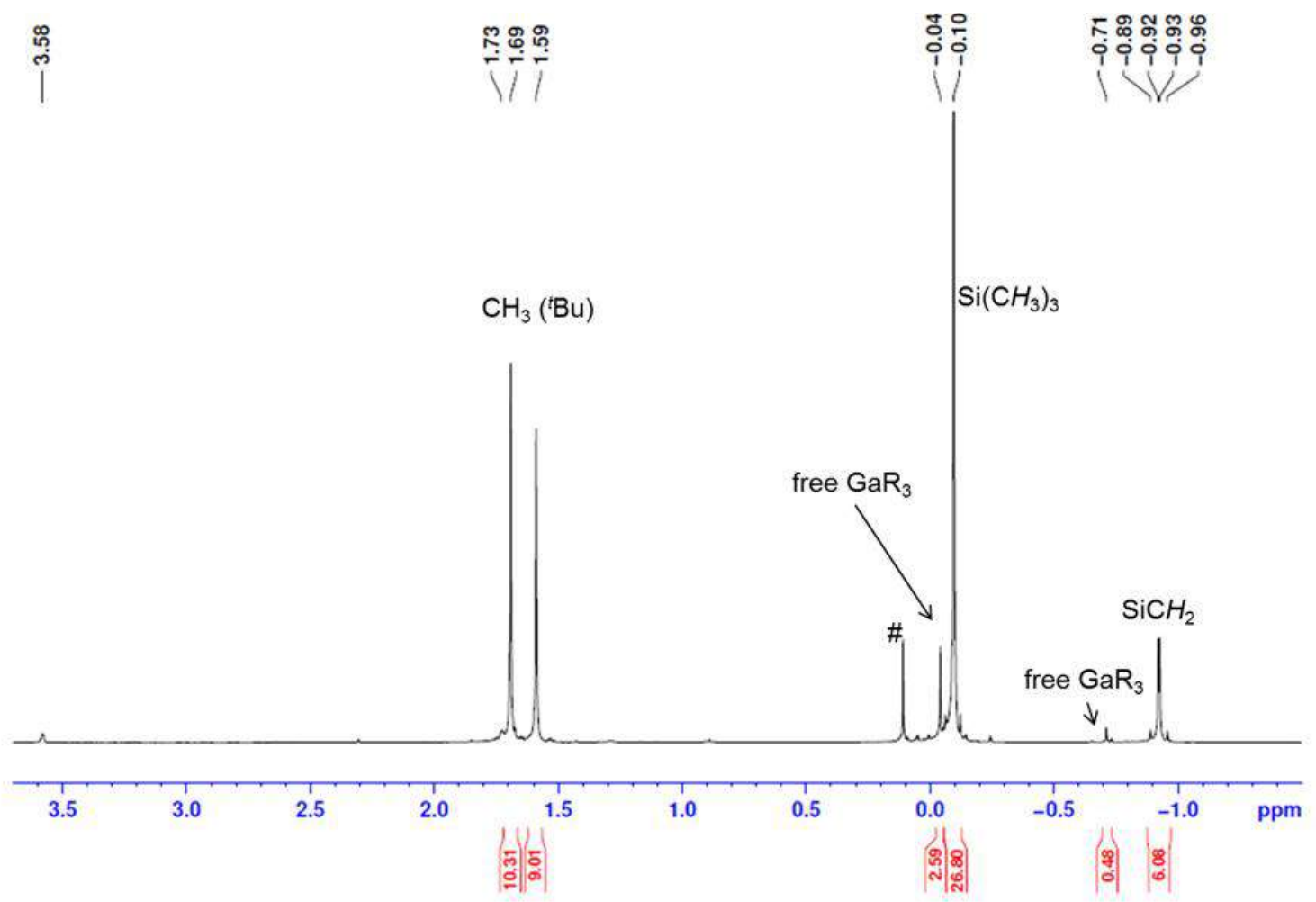

Figure S34: High field region of ${ }^{1} \mathrm{H}$ NMR spectrum of $\mathbf{3}$ in $\mathrm{d}_{8}$-THF (\# = grease)

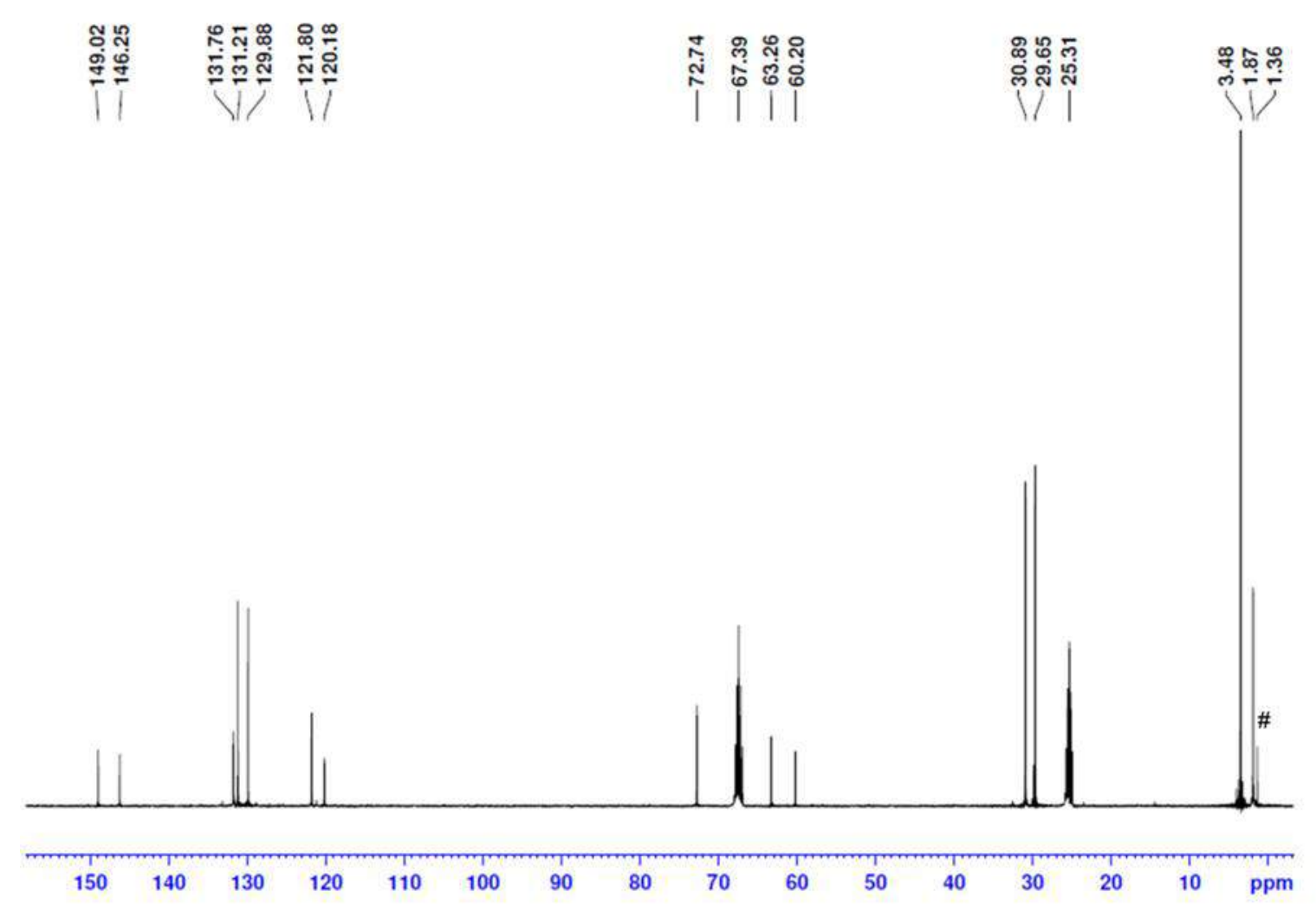

Figure S35: ${ }^{13} \mathrm{C}$ NMR spectrum of 3 in $\mathrm{d}_{8}$ THF (\# = grease). 


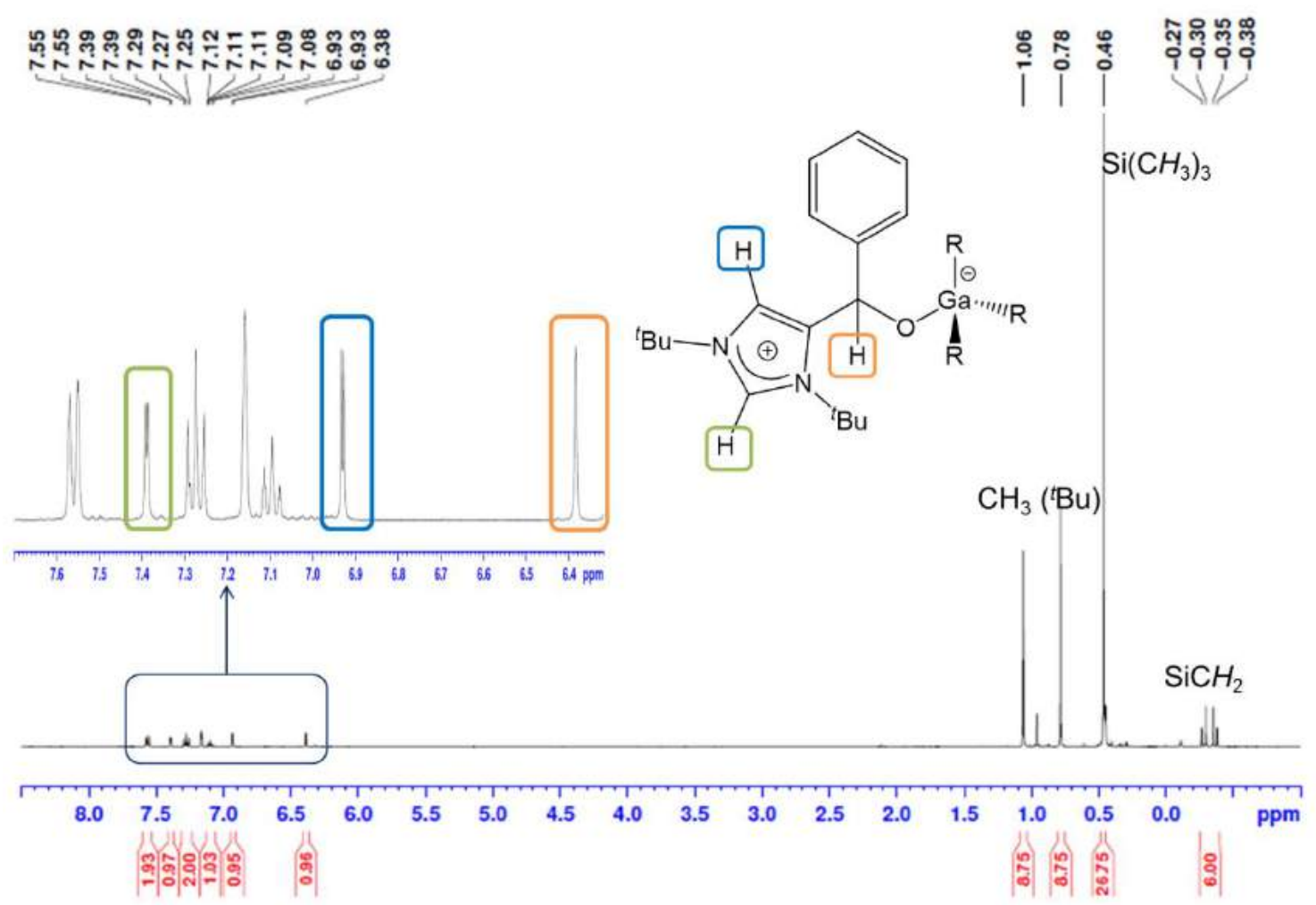

Figure S36: ${ }^{1} \mathrm{H}$ NMR spectrum of 4 in $\mathrm{C}_{6} \mathrm{D}_{6}$.

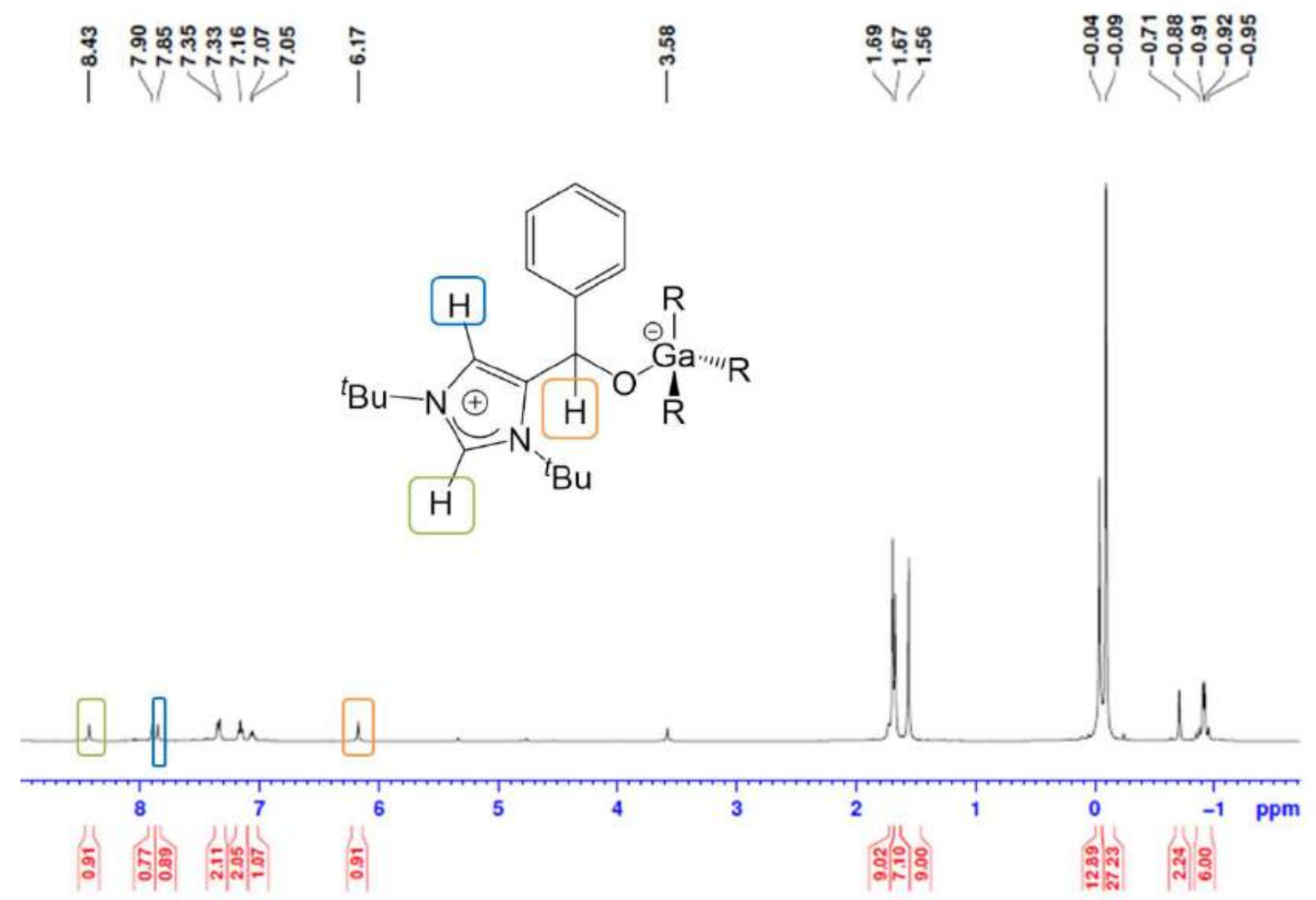

Figure S37: ${ }^{1} \mathrm{H}$ NMR spectrum of 4 in $\mathrm{d}_{8}$-THF. 


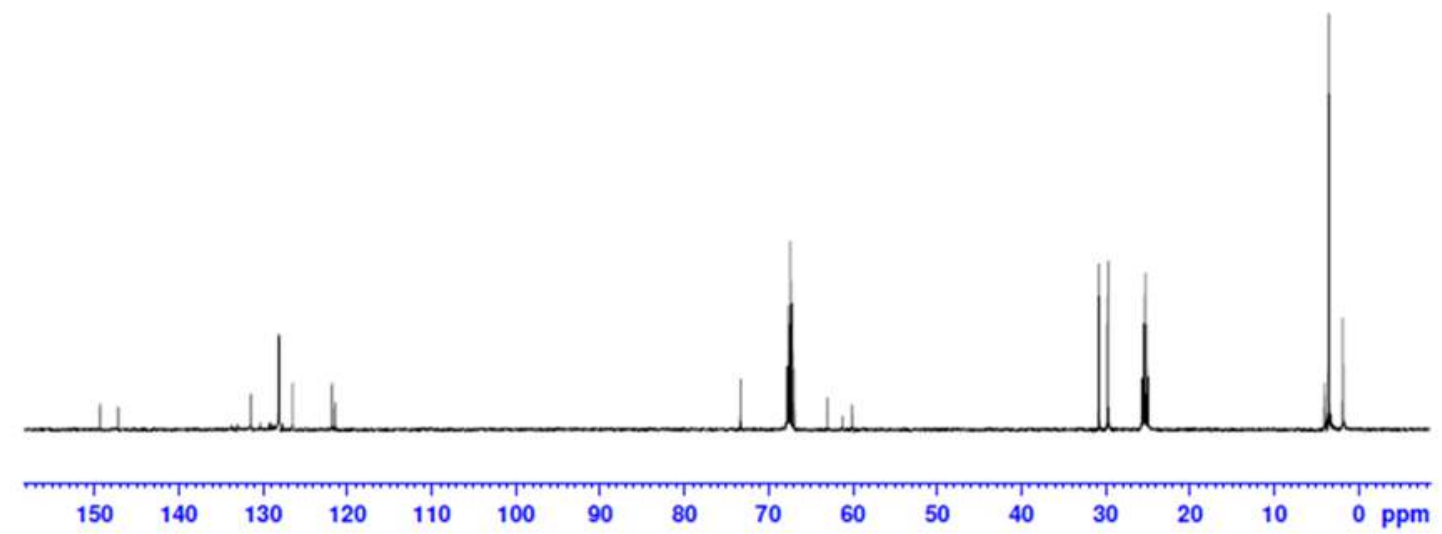

Figure S38: ${ }^{13} \mathrm{C}$ NMR spectrum of 4 in $\mathrm{d}_{8}$-THF.

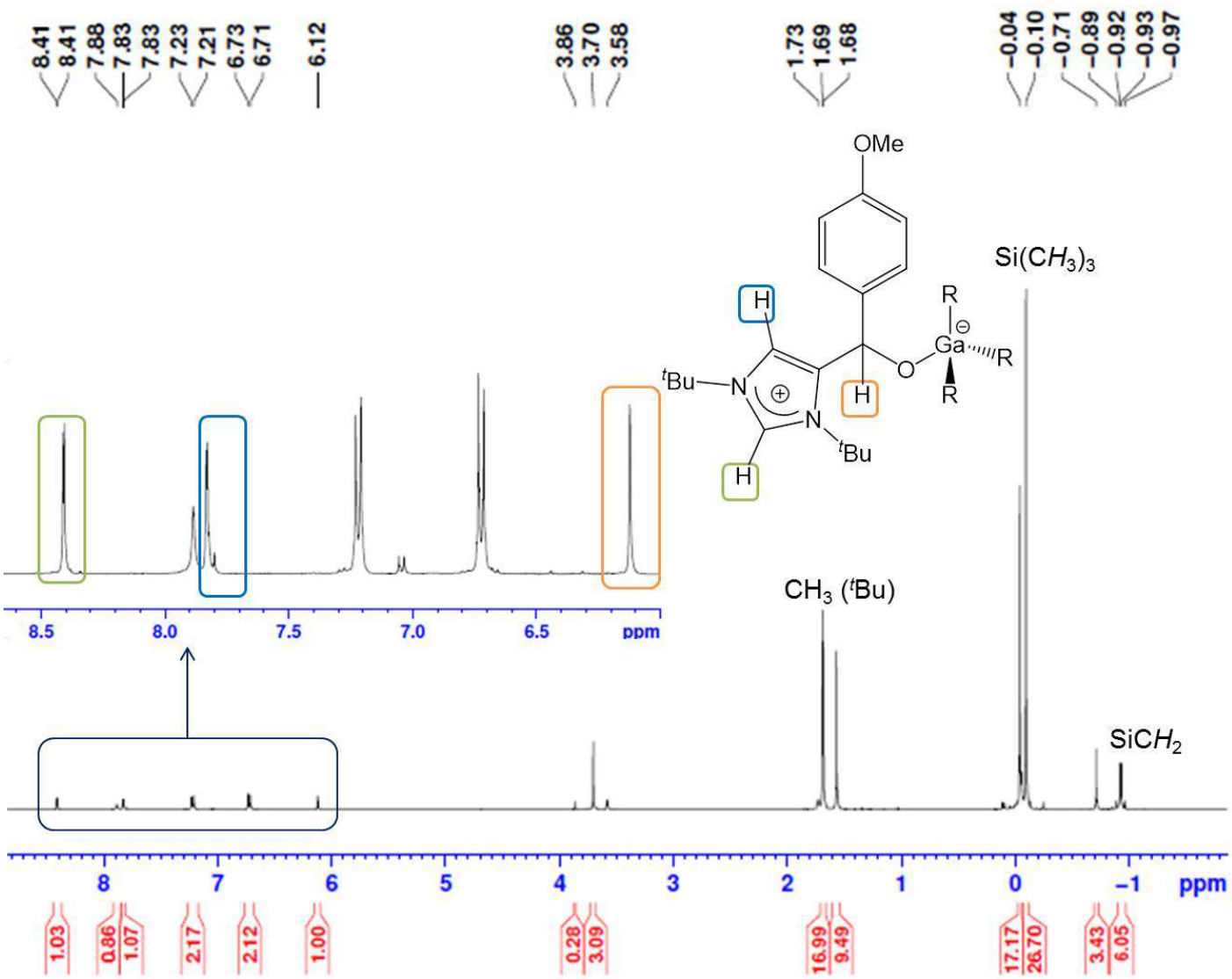

Figure S39: ${ }^{1} \mathrm{H}$ NMR spectrum of 5 in $\mathrm{d}_{8}$-THF. 


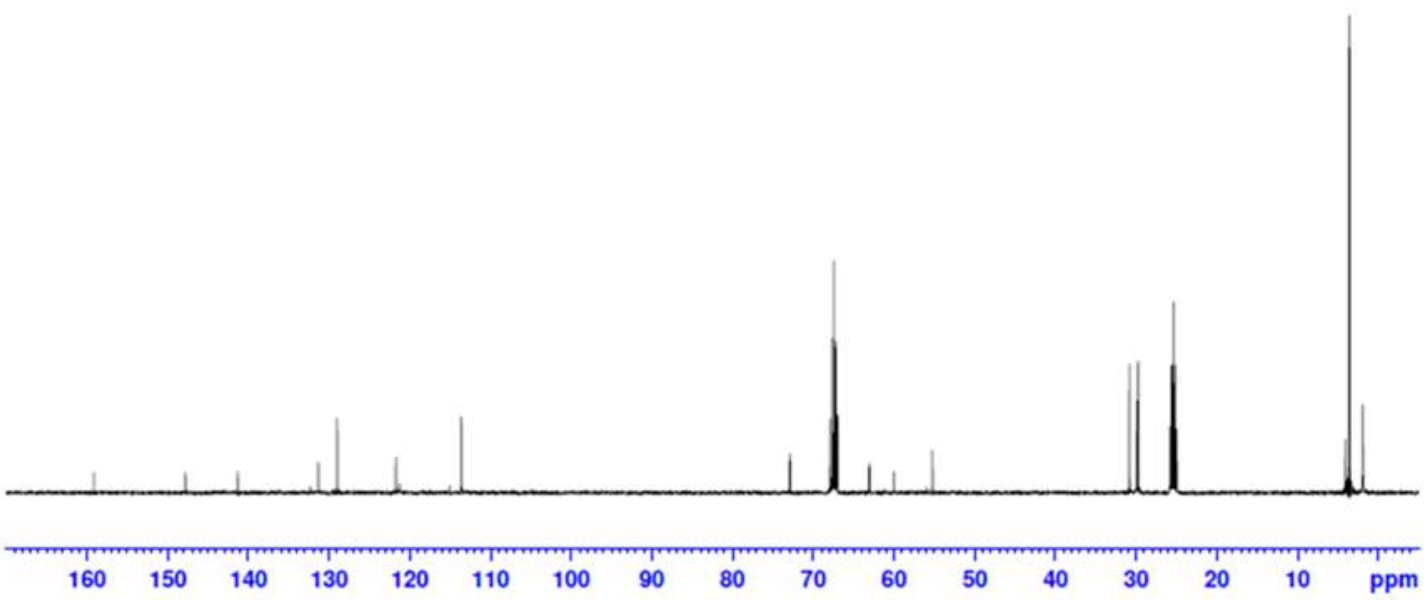

Figure S40: ${ }^{13} \mathrm{C}$ NMR spectrum of 5 in $\mathrm{d}_{8}$-THF.

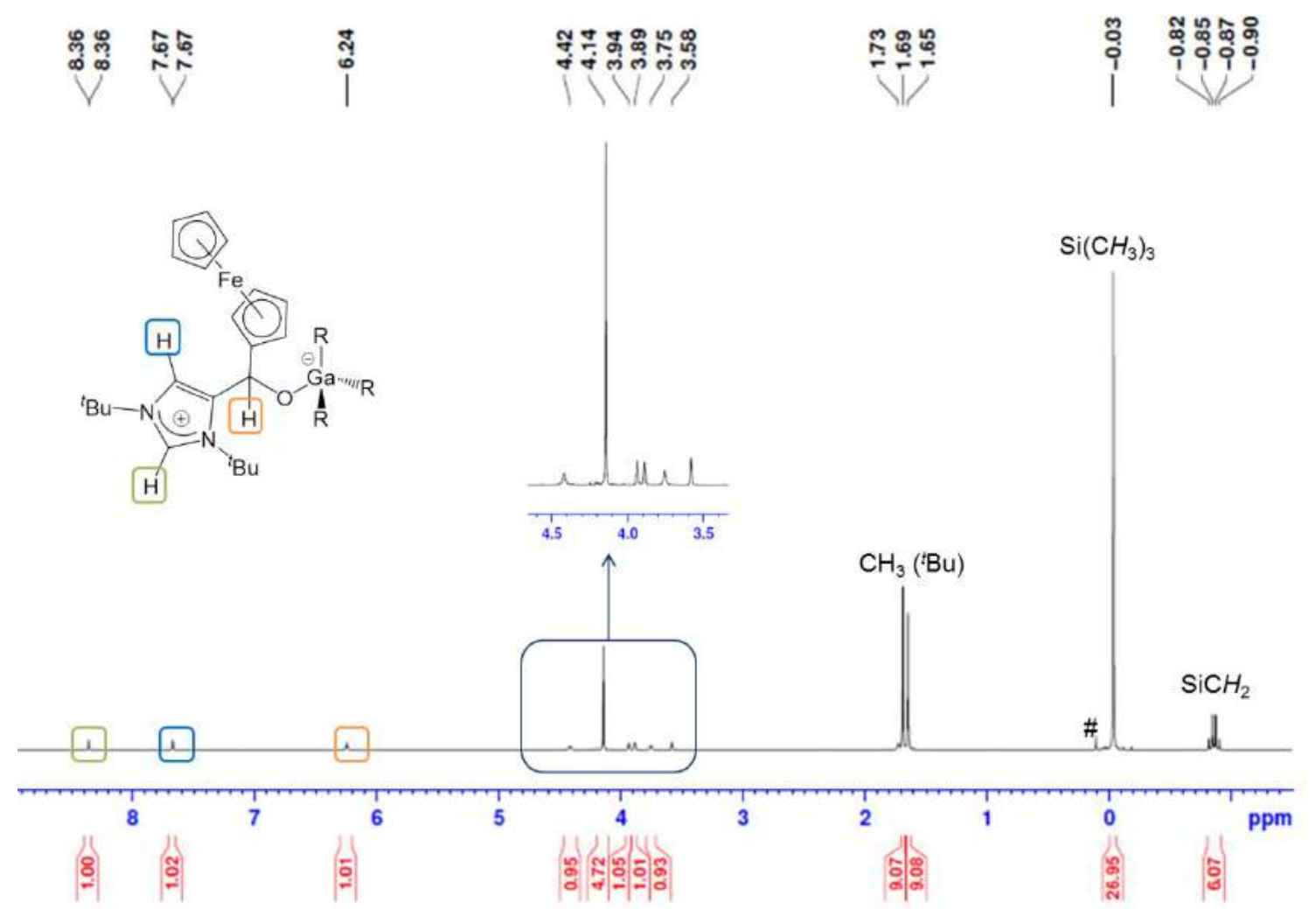

Figure S41: ${ }^{1} \mathrm{H}$ NMR spectrum of 6 in $d_{8}$-THF. 


إ |

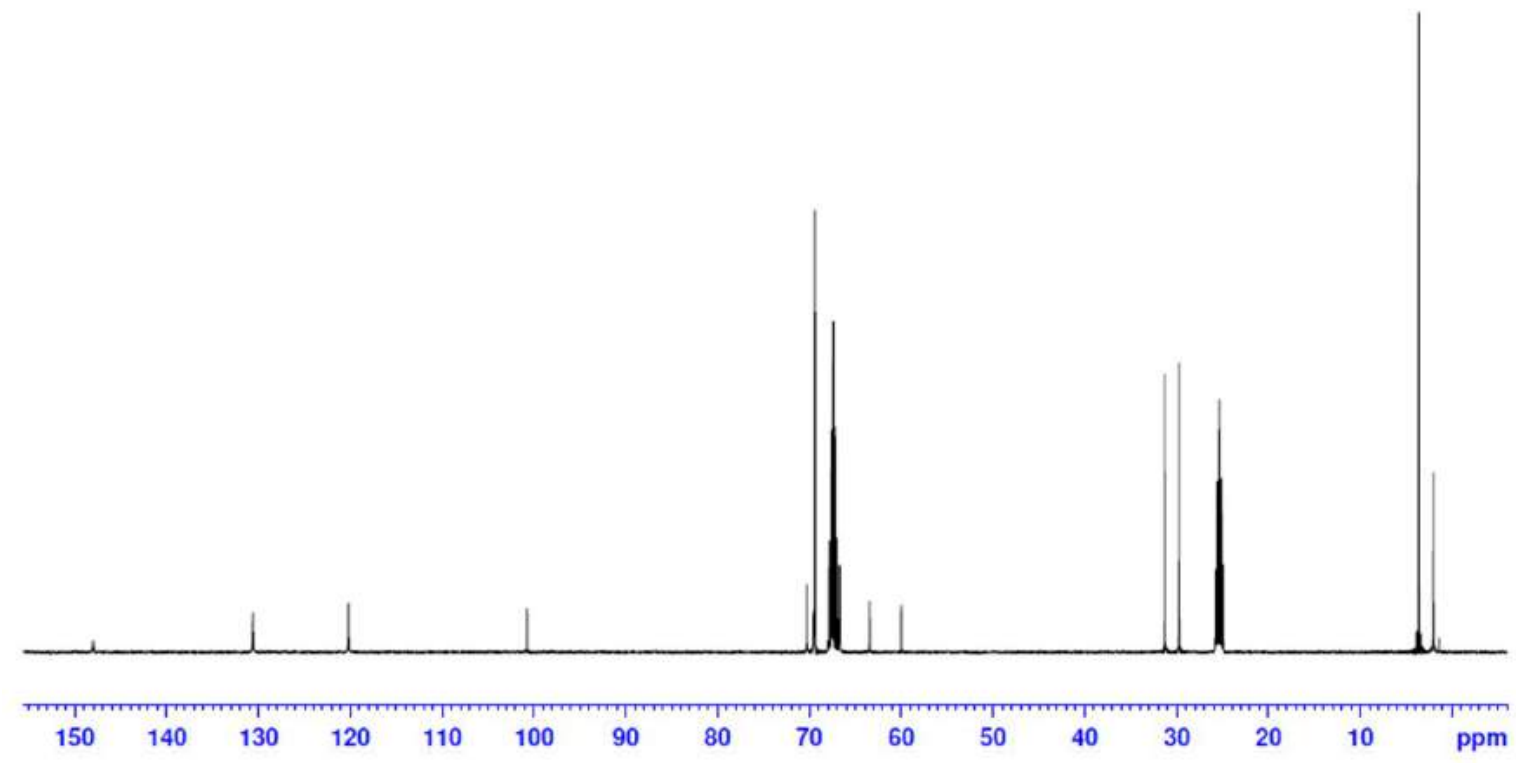

Figure S42: ${ }^{13} \mathrm{C}$ NMR spectrum of $\boldsymbol{6}$ in $\mathrm{d}_{8}$-THF.

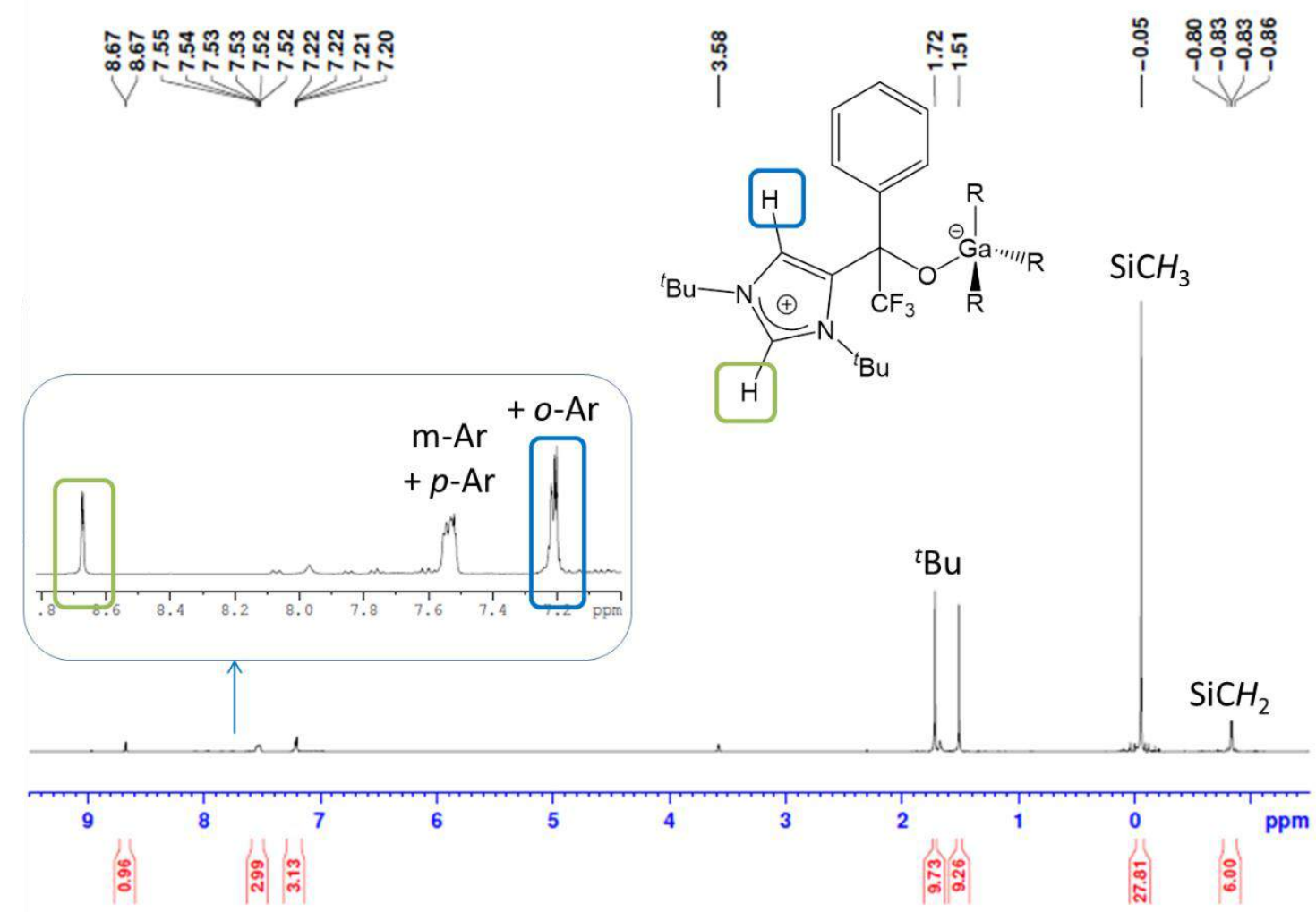

Figure S43: ${ }^{1} \mathrm{H}$ NMR spectrum of 7 in $\mathrm{d}_{8}$-THF. 


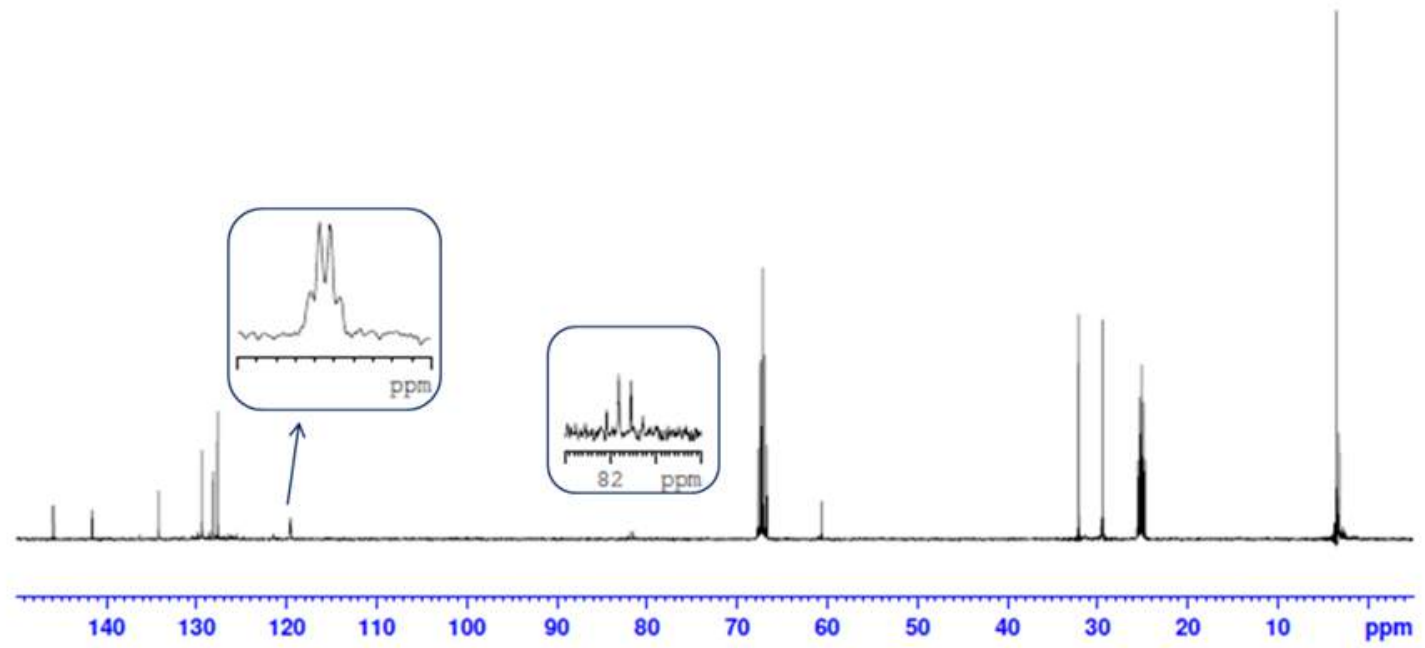

Figure S44: ${ }^{13} \mathrm{C}$ NMR spectrum of 7 in $\mathrm{d}_{8}$-THF.
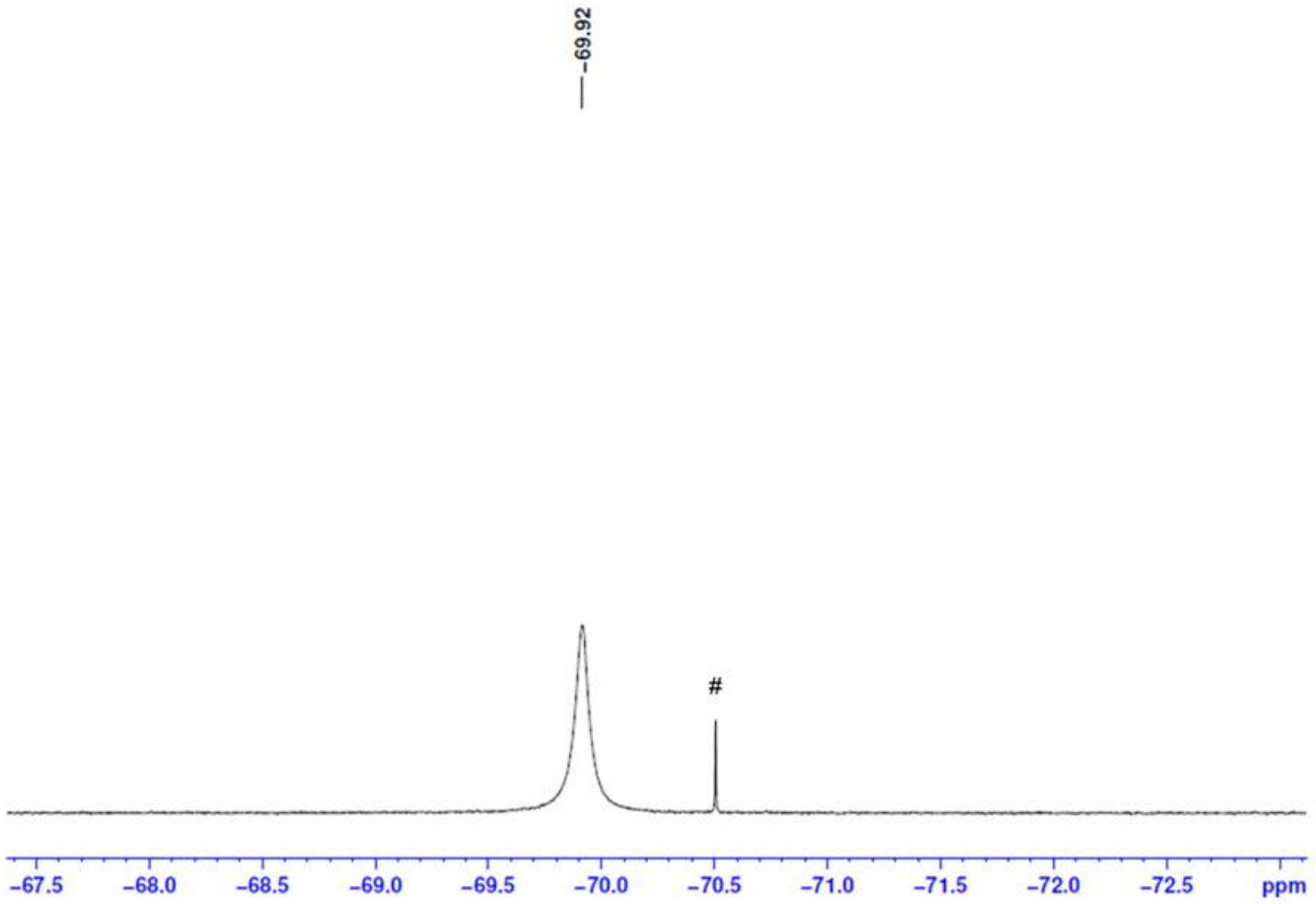

Figure S45: ${ }^{19} \mathrm{~F}$ NMR spectrum of 7 in $\mathrm{d}_{8}-\mathrm{THF}(\#=$ impurity). 


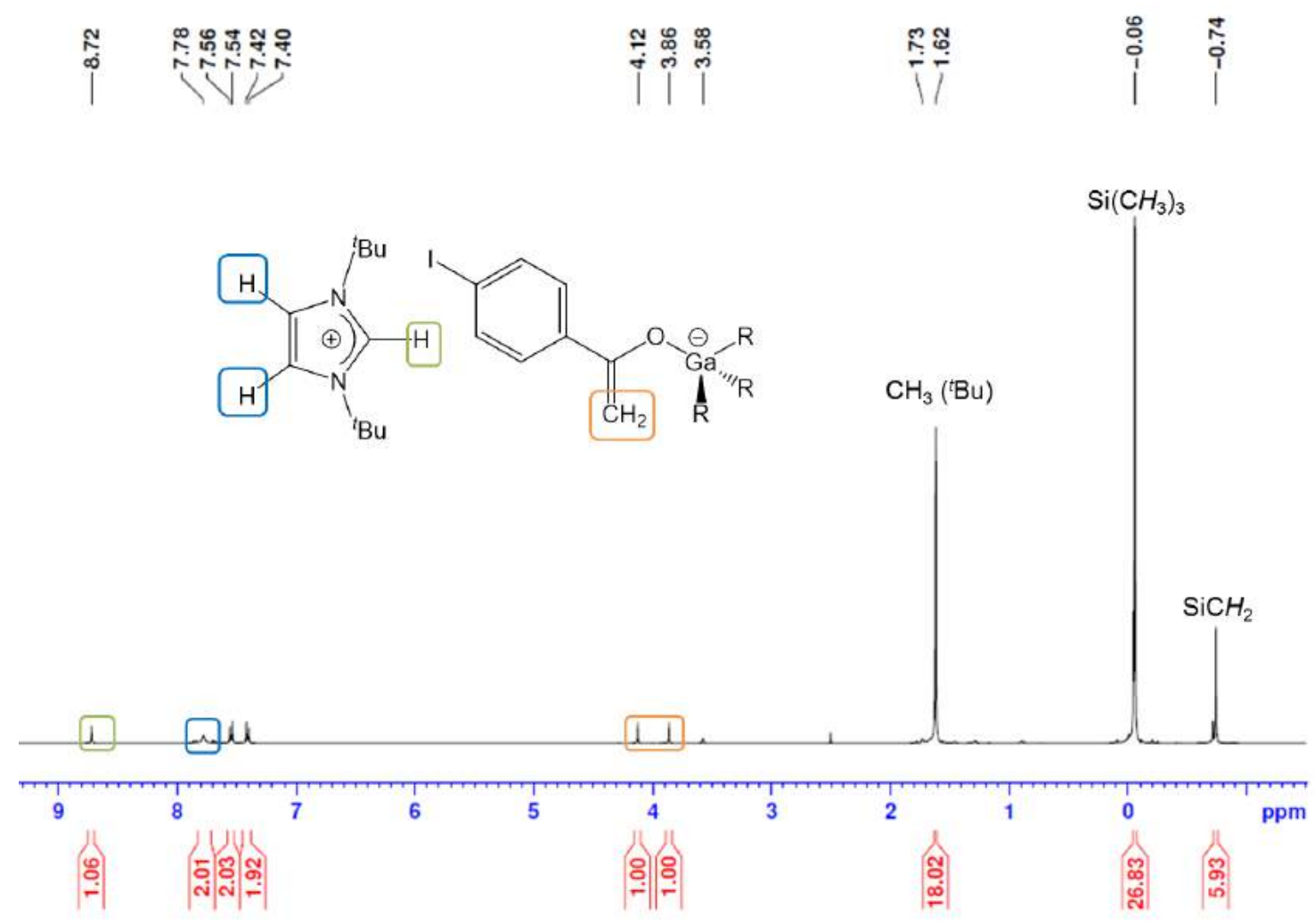

Figure S46: ${ }^{1} \mathrm{H}$ NMR spectrum of $\mathbf{8}$ in $\mathrm{d}_{8}$-THF.

ণ্ֶু

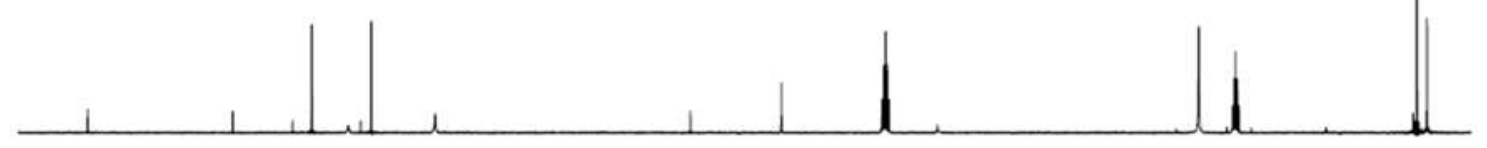

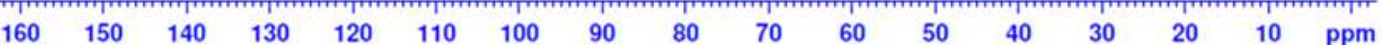

Figure S47: ${ }^{13} \mathrm{C}$ NMR spectrum of 8 in $\mathrm{d}_{8}$-THF. 

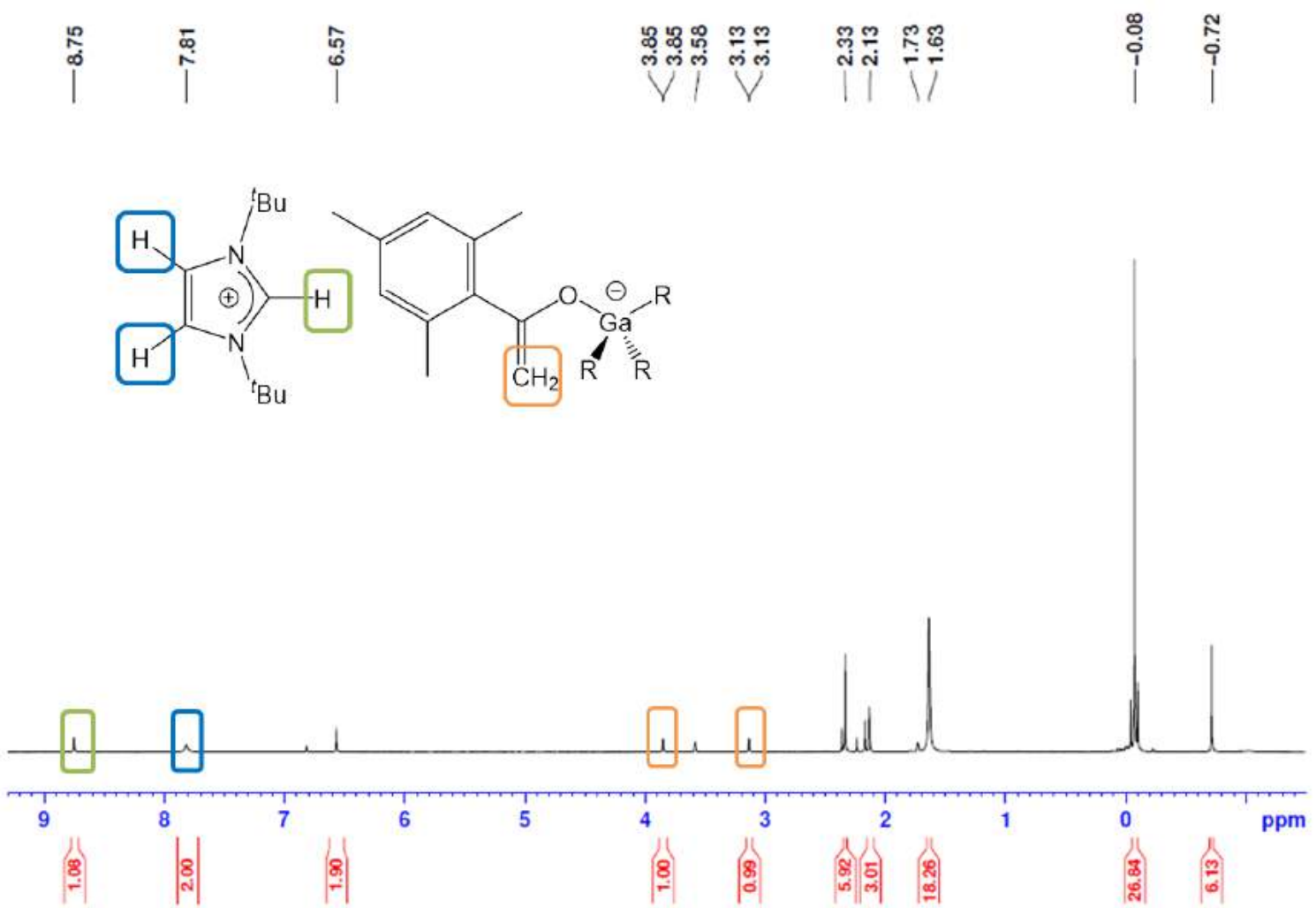

Figure S48: ${ }^{1} \mathrm{H}$ NMR spectrum of 9 in $\mathrm{d}_{8}$-THF.

总

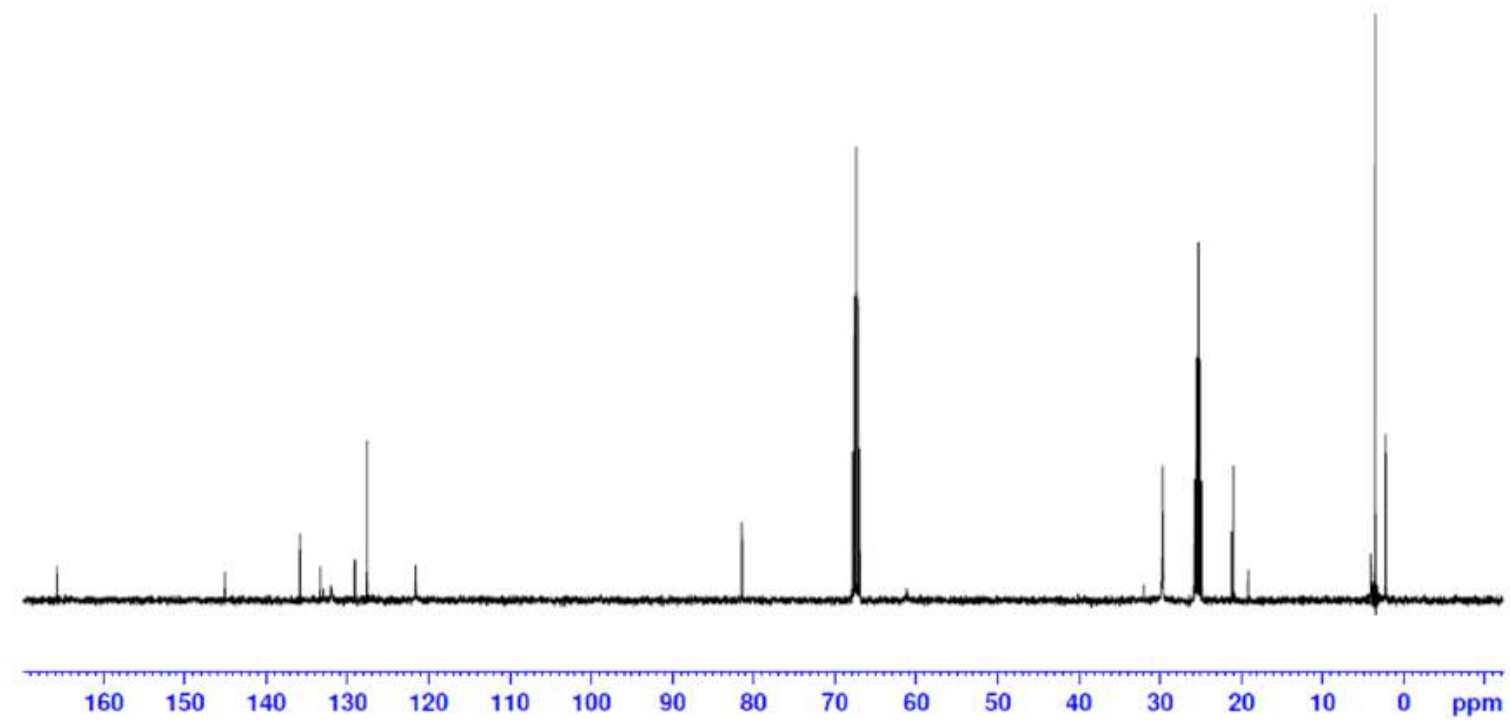

Figure S49: ${ }^{13} \mathrm{C}$ NMR spectrum of 9 in $\mathrm{d}_{8}$-THF. 


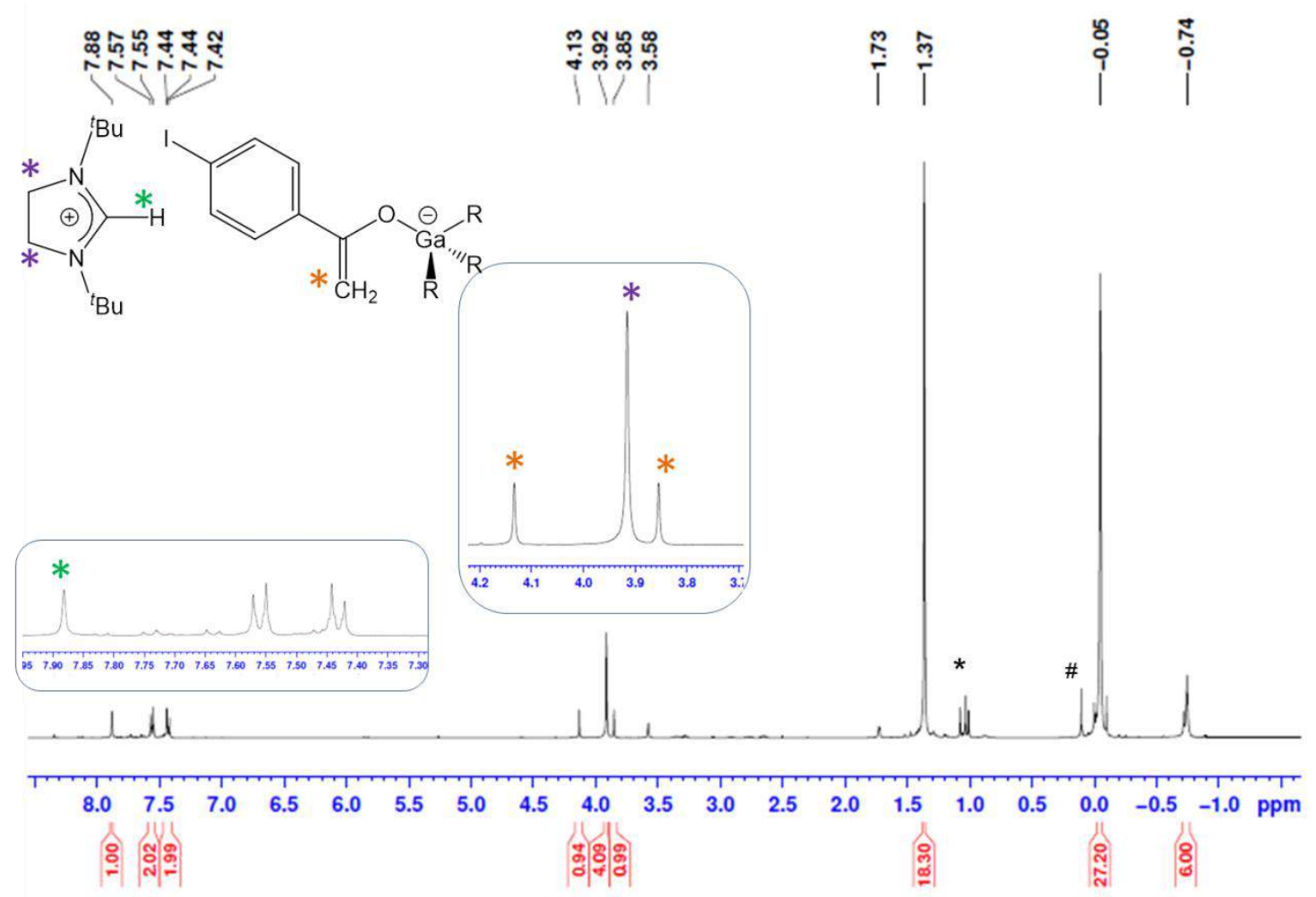

Figure S50: ${ }^{1} \mathrm{H}$ NMR spectrum of $\mathbf{1 0}$ in $\mathrm{d}_{8}$-THF.
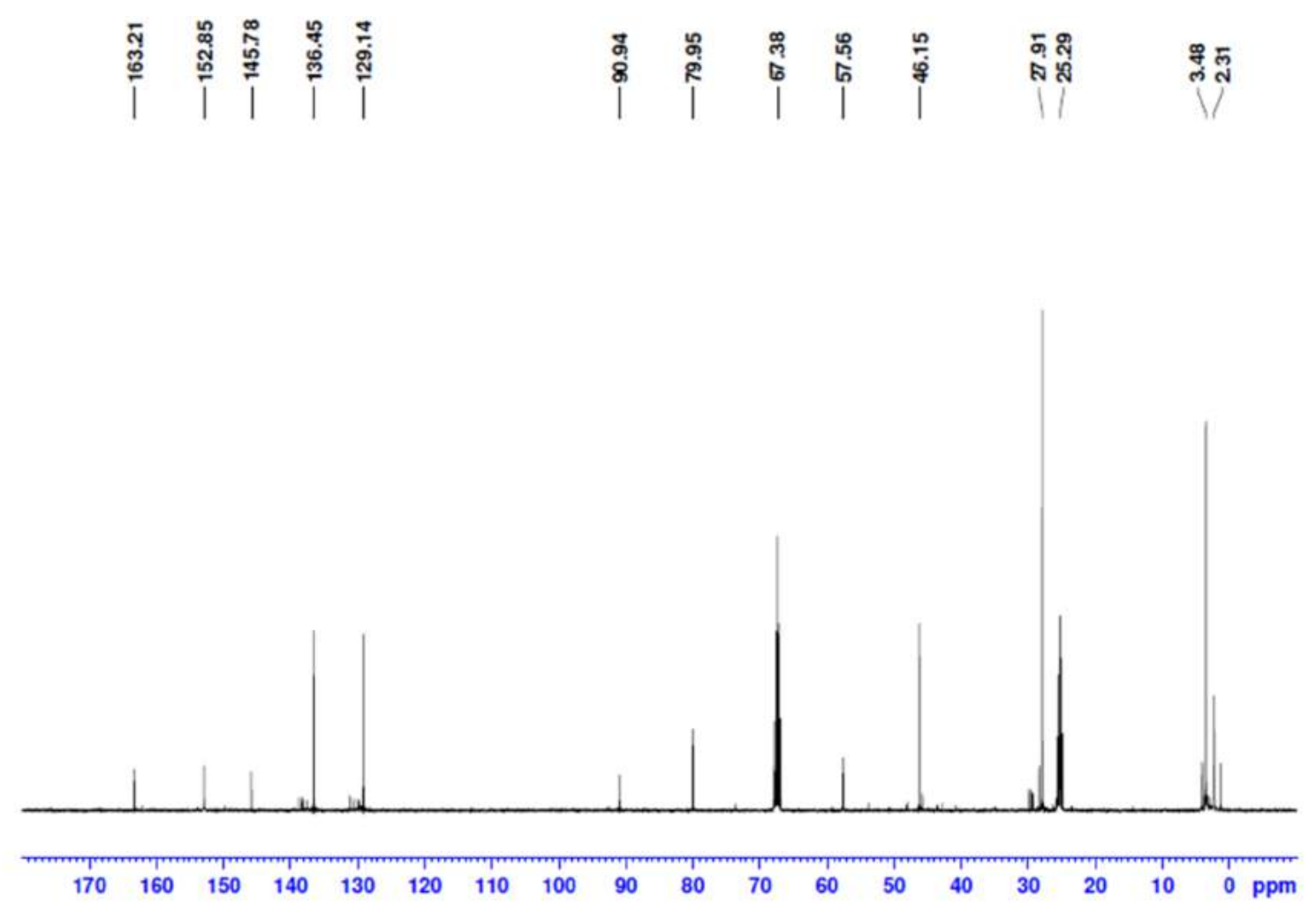

Figure S51: ${ }^{13} \mathrm{C}$ NMR spectrum of $\mathbf{1 0}$ in $\mathrm{d}_{8}$-THF. 

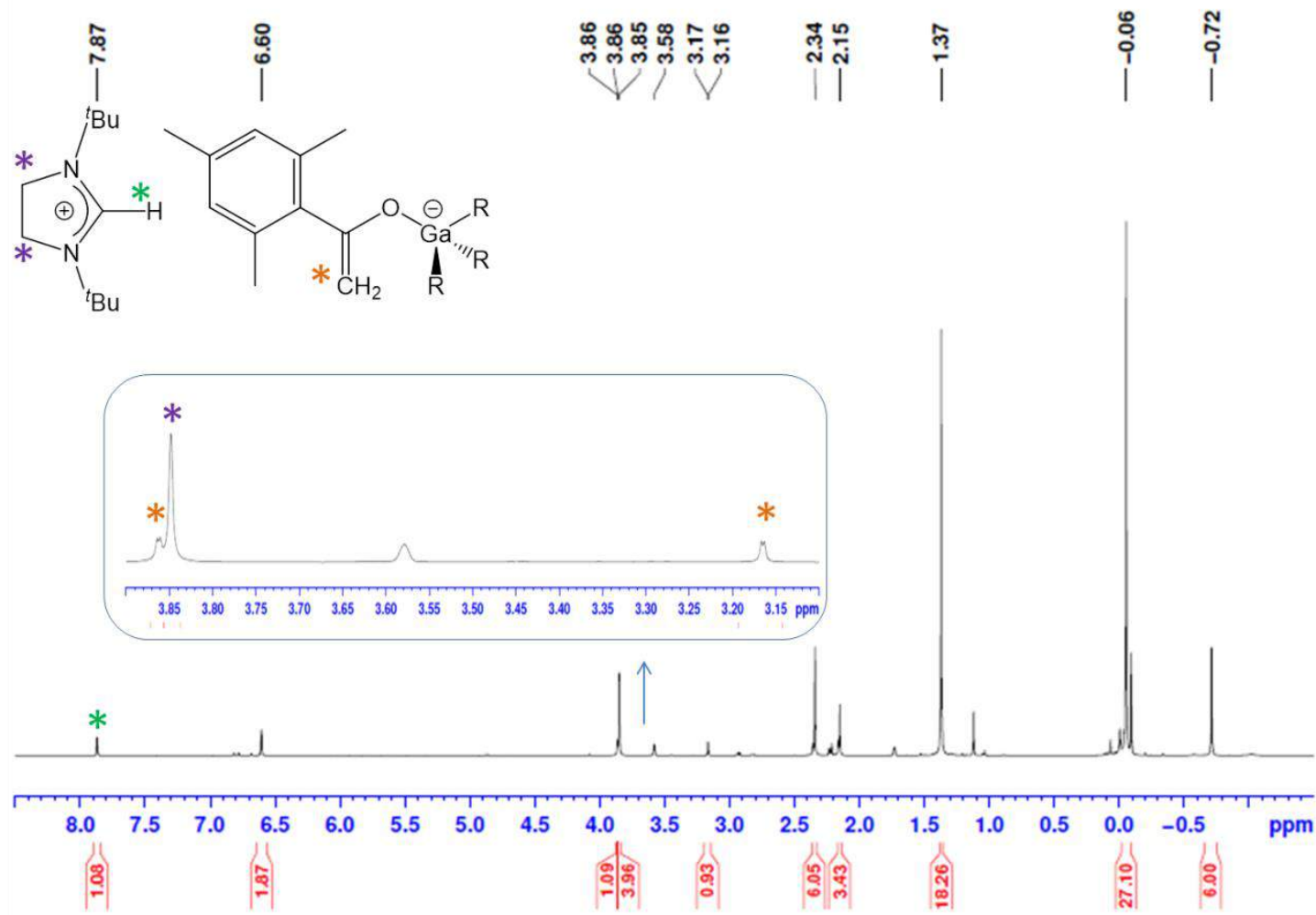

Figure S52: ${ }^{1} \mathrm{H}$ NMR spectrum of $\mathbf{1 1}$ in $\mathrm{d}_{8}$-THF
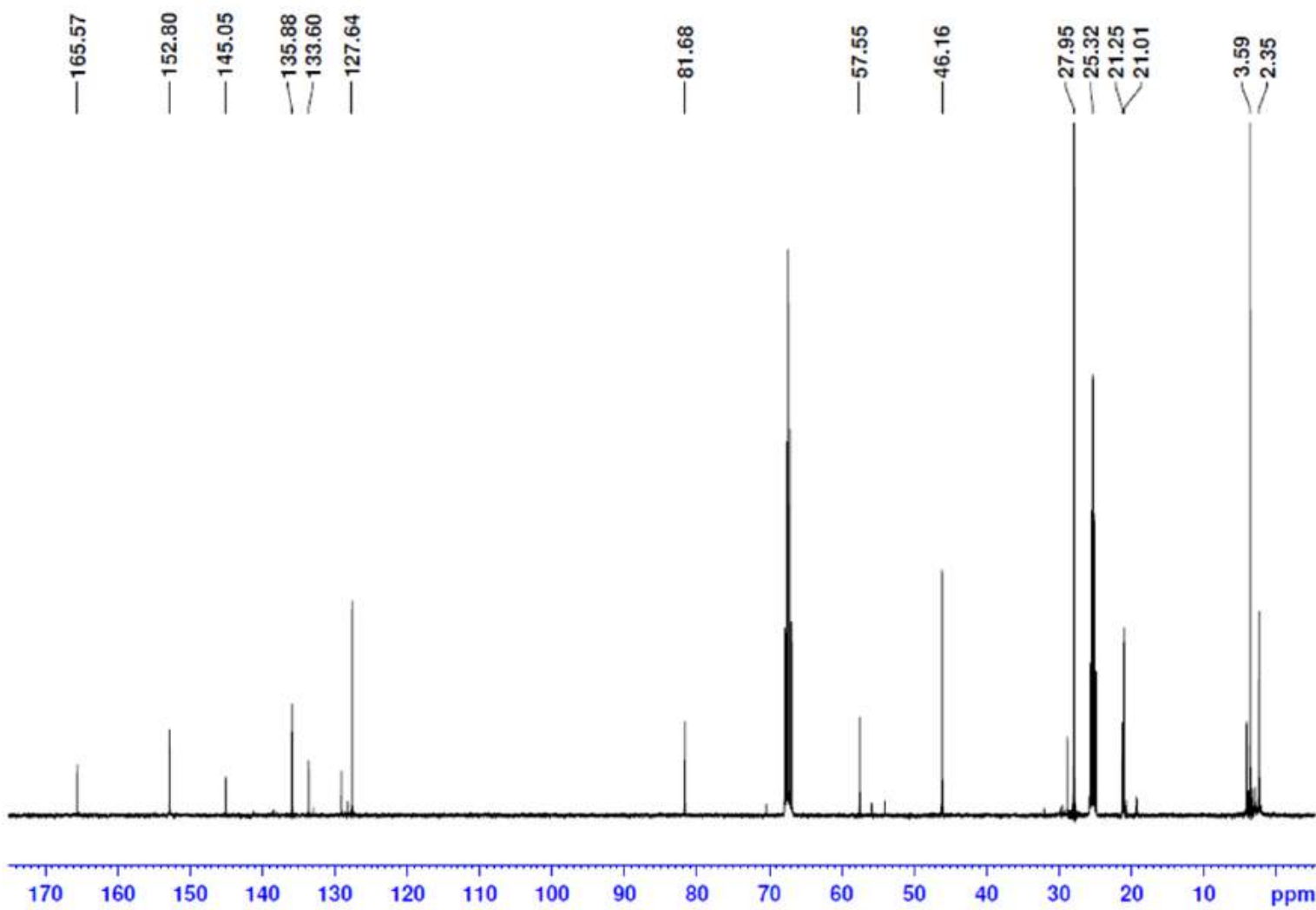

Figure S53: ${ }^{13} \mathrm{C}$ NMR spectrum of $\mathbf{1 1}$ in $\mathrm{d}_{8}$-THF 


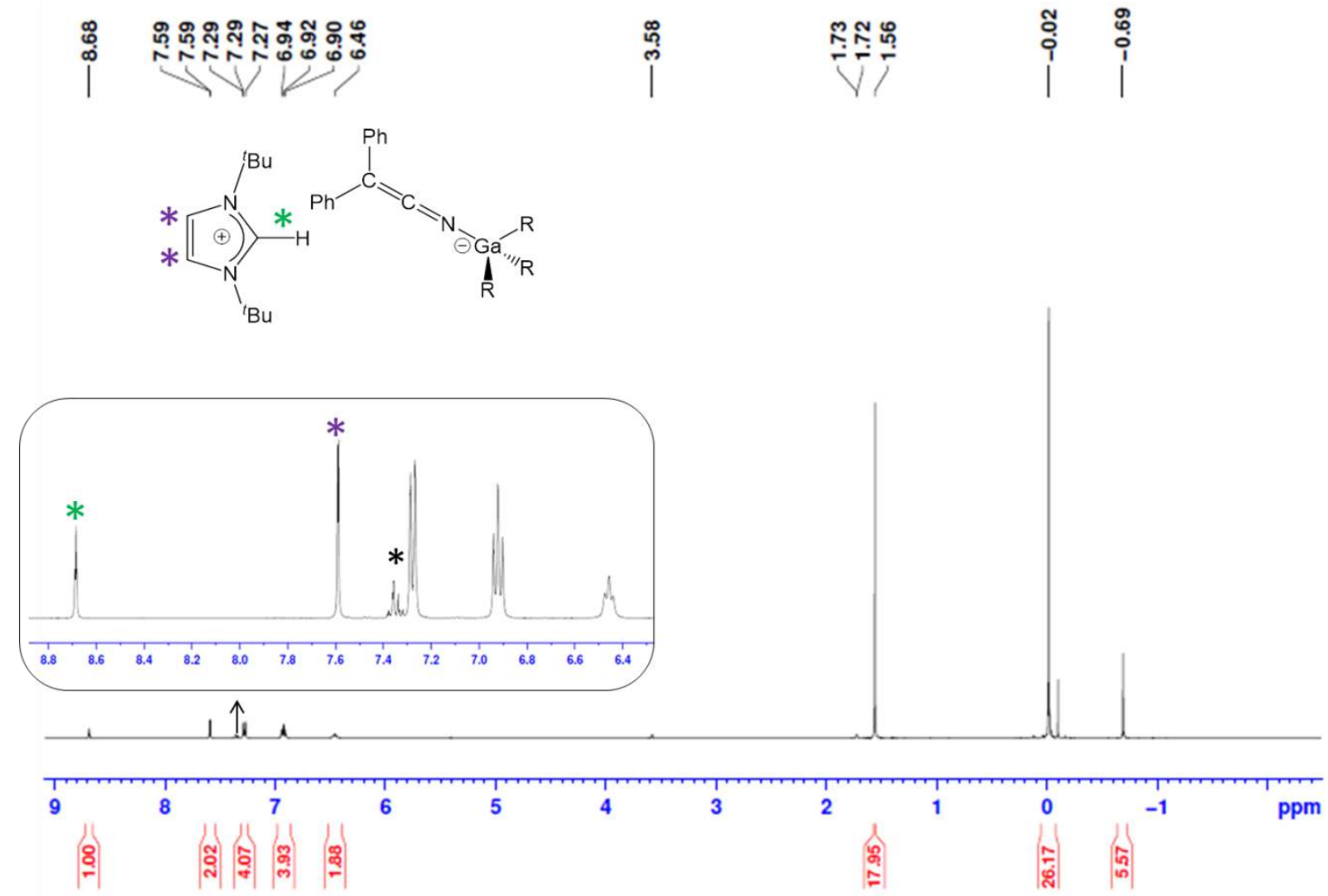

Figure S54: ${ }^{1} \mathrm{H}$ NMR spectrum of 12 in $\mathrm{d}_{8}$-THF.
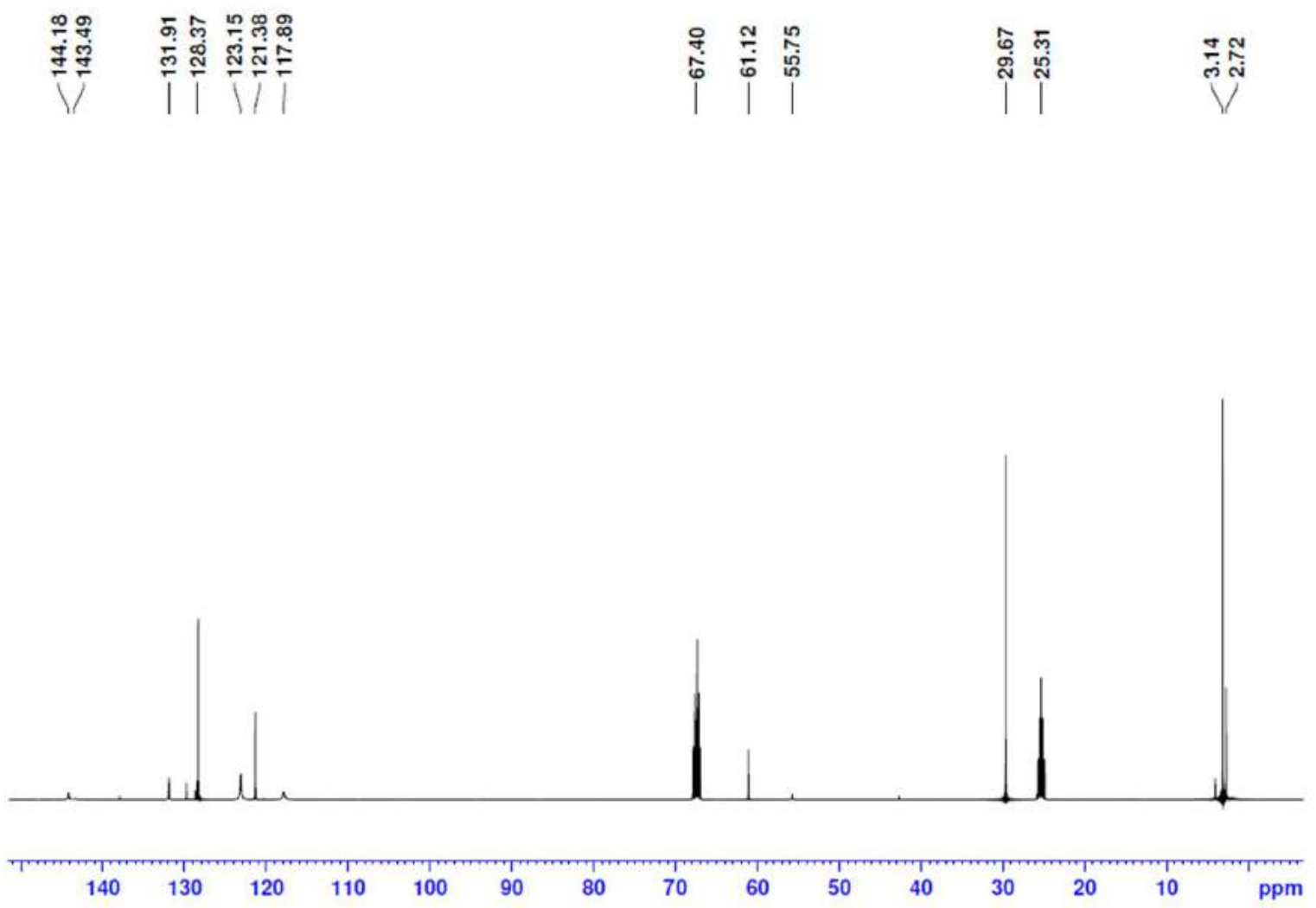

Figure S55: ${ }^{13} \mathrm{C}$ NMR spectrum of $\mathbf{1 2}$ in $\mathrm{d}_{8}$-THF. 


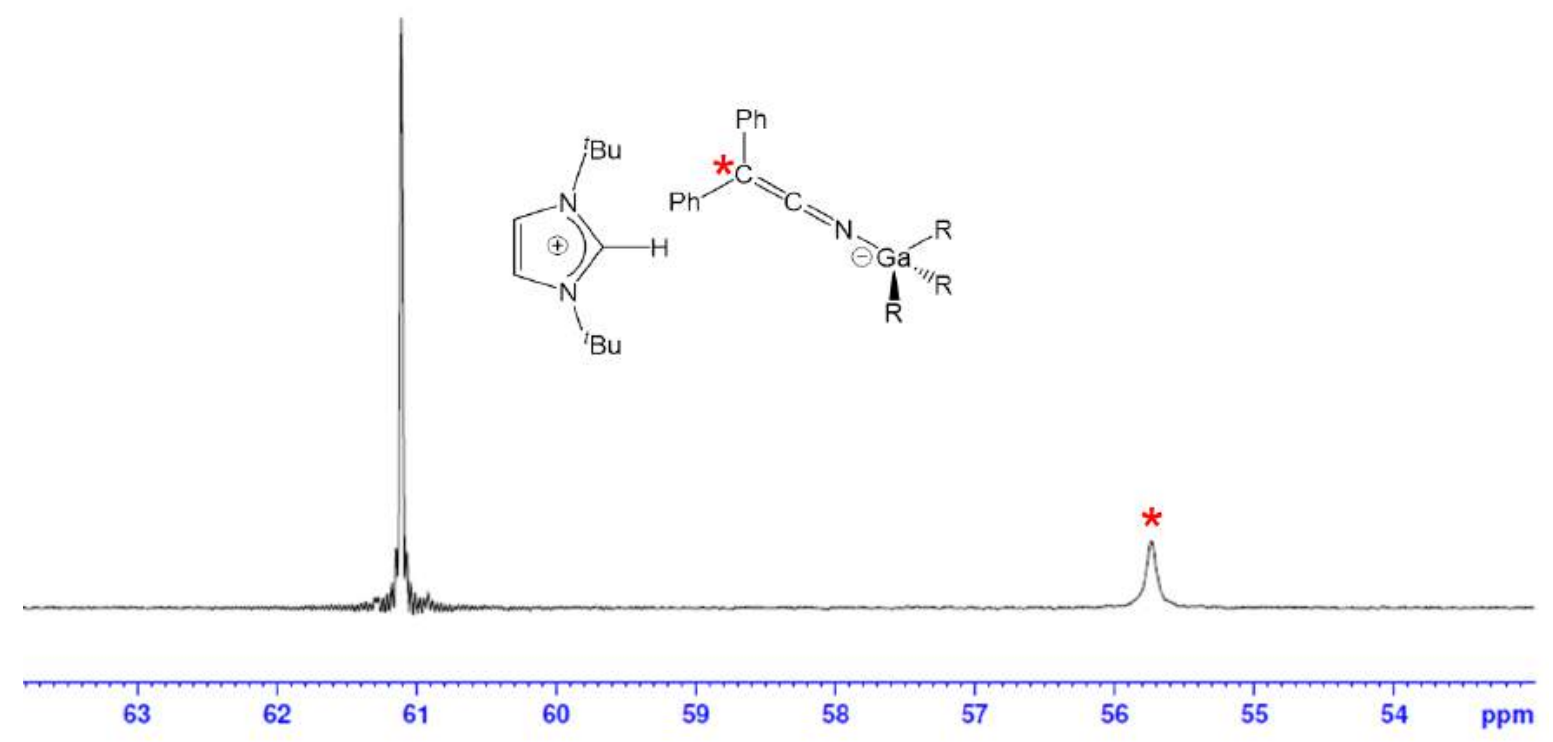

Figure S56: Part of the ${ }^{13} \mathrm{C}$ NMR spectrum of 12 in $\mathrm{d}_{8}$-THF.

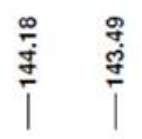
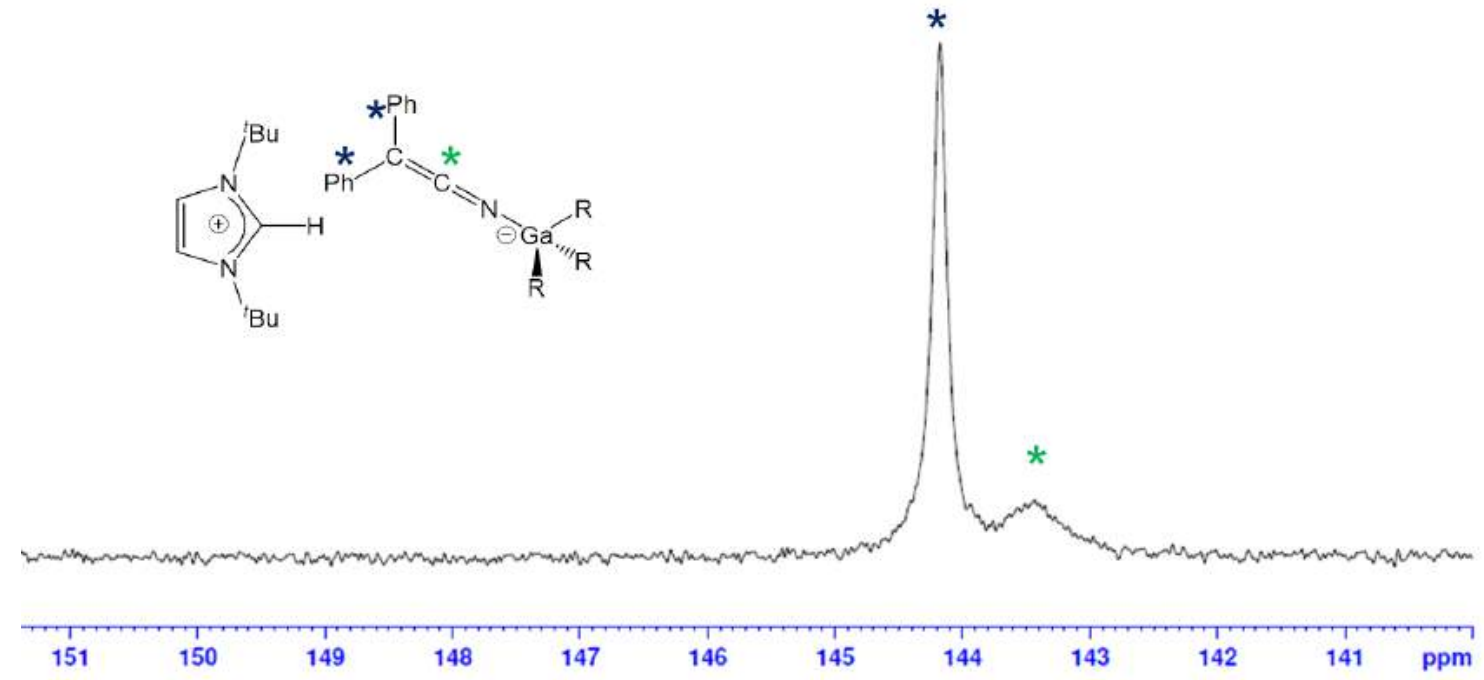

Figure S57: Part of the ${ }^{13} \mathrm{C}$ NMR spectrum of $\mathbf{1 2}$ in $\mathrm{d}_{8}$-THF. 


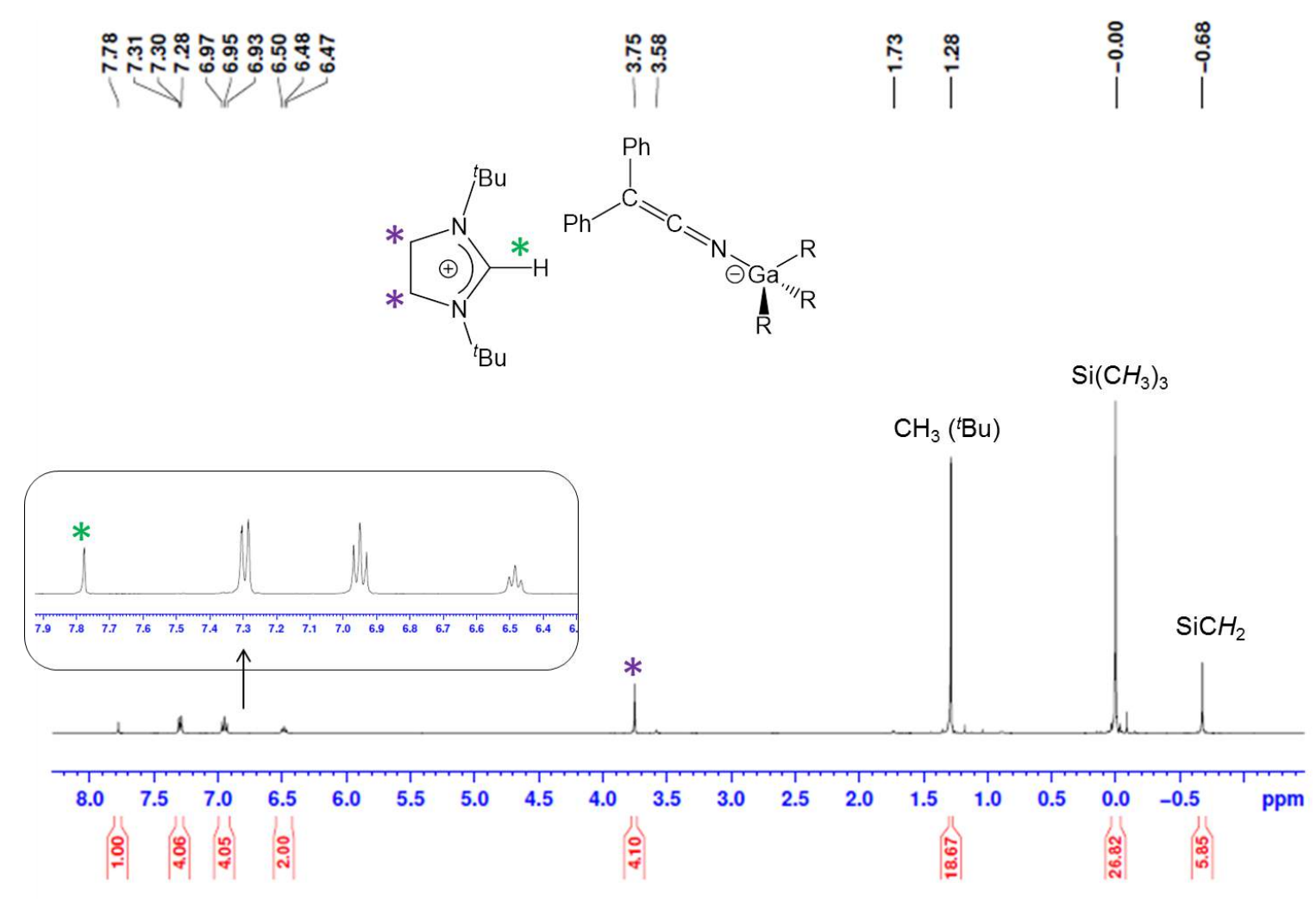

Figure S58: ${ }^{1} \mathrm{H}$ NMR spectrum of $\mathbf{1 3}$ in $\mathrm{d}_{8}$-THF

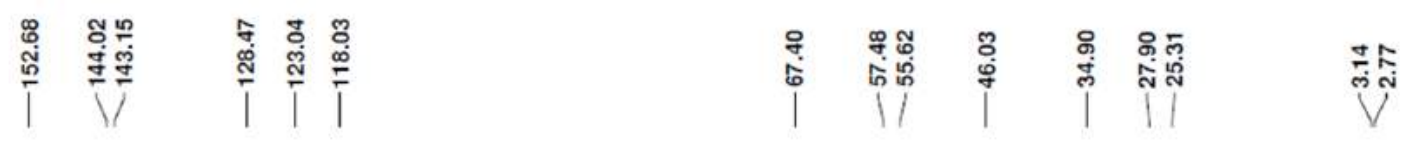

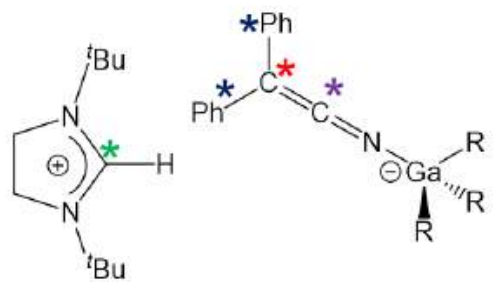
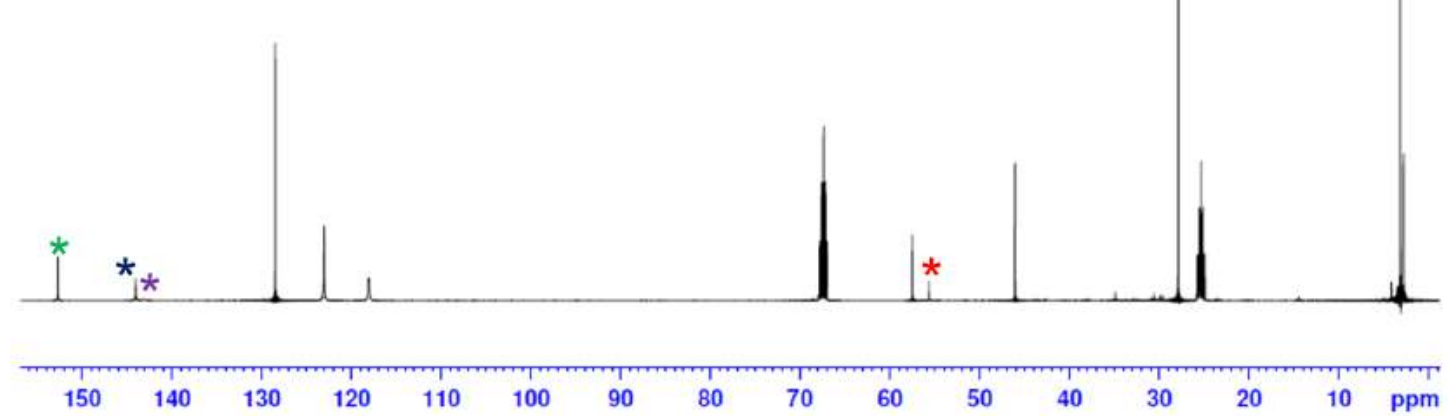

Figure S59: ${ }^{13} \mathrm{C}$ NMR spectrum of $\mathbf{1 3}$ in $\mathrm{d}_{8}$-THF. 


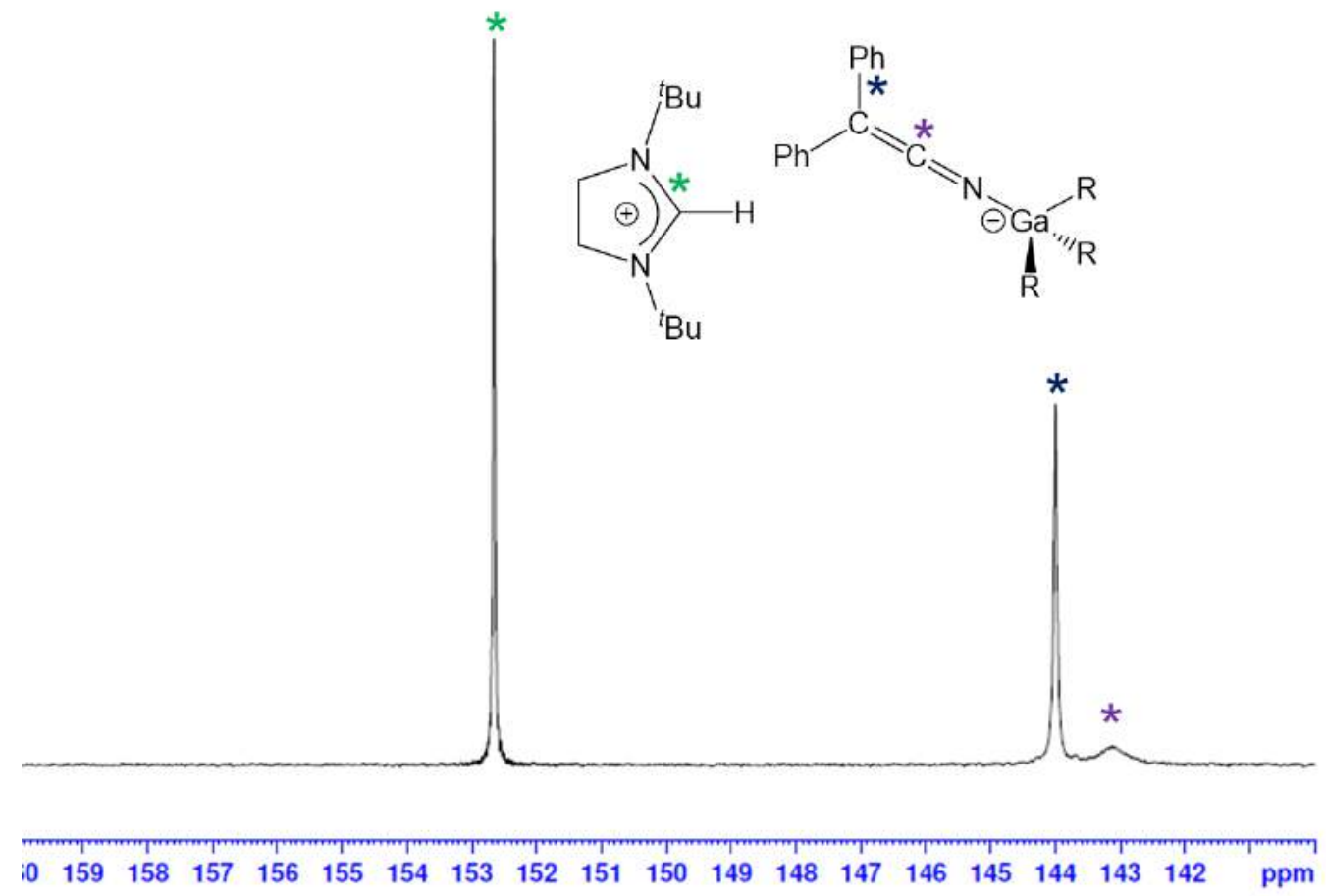

Figure S60: Part of the ${ }^{13} \mathrm{C}$ NMR spectrum of $\mathbf{1 3}$ in $\mathrm{d}_{8}$-THF.

\section{IR spectra of products 12 and 13}




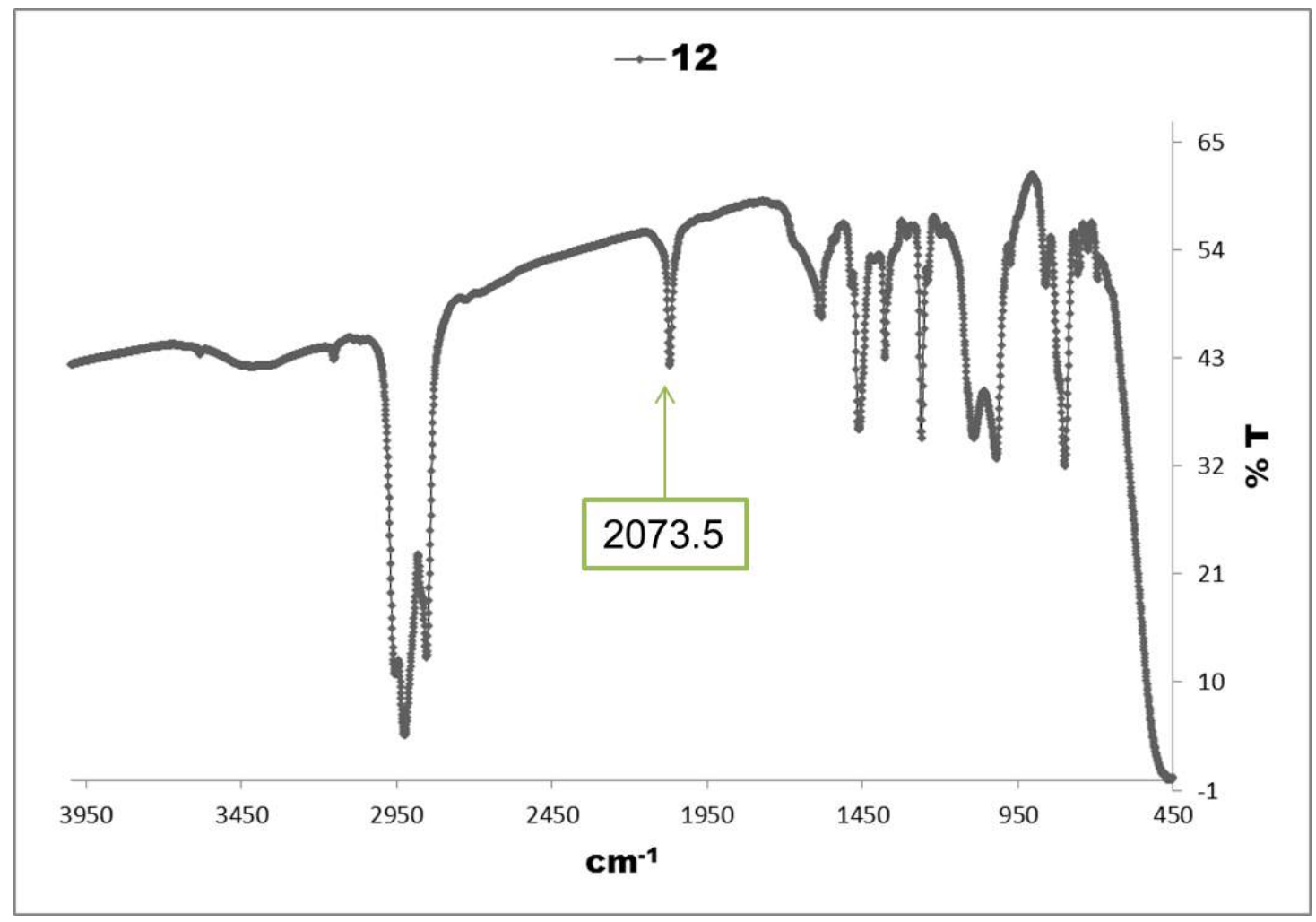

Figure S61: IR spectrum of 12.

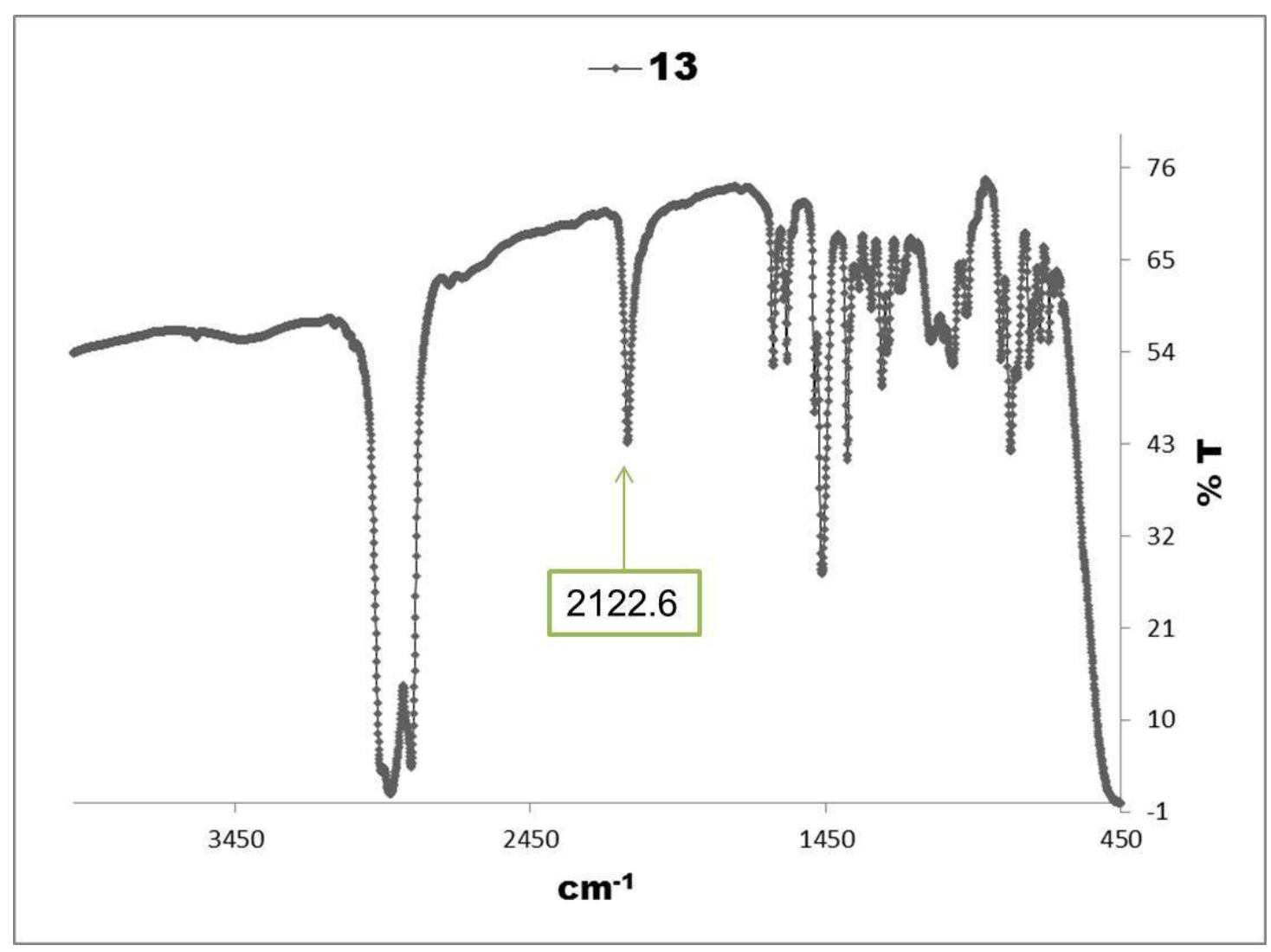

Figure S62: IR spectrum of 13. 


\section{Cartesian coordinates}

1. Model $\mathrm{II}_{\mathrm{Ar}}$

\begin{tabular}{|c|c|c|c|}
\hline $\mathrm{Ga}$ & -0.769709744 & -1.5834316297 & 0.452434623 \\
\hline $\mathrm{Br}$ & 4.6348981809 & 2.9897164869 & -4.01971941 \\
\hline $\mathrm{Si}$ & -2.9880231785 & -2.1989858598 & 3.0296015771 \\
\hline $\mathrm{Si}$ & 2.1335331946 & -3.4641637875 & 0.5074106799 \\
\hline $\mathrm{Si}$ & -2.4822139714 & -2.9039568733 & -2.2063337462 \\
\hline $\mathrm{O}$ & 0.3688177787 & 0.0780865345 & 0.5387272417 \\
\hline $\mathrm{N}$ & 0.166557355 & 2.662612761 & 2.0813609496 \\
\hline $\mathrm{N}$ & -1.4921955753 & 3.1292749601 & 0.7142150878 \\
\hline $\mathrm{C}$ & -0.3884361813 & 2.3416870829 & 0.8797721747 \\
\hline $\mathrm{C}$ & -0.6149900936 & 3.6375254626 & 2.6717190728 \\
\hline $\mathrm{H}$ & -0.3968836153 & 4.0531565538 & 3.6349372254 \\
\hline $\mathrm{C}$ & -1.6305830742 & 3.9285889103 & 1.8341765509 \\
\hline $\mathrm{H}$ & -2.4254239126 & 4.6364040814 & 1.9569148973 \\
\hline $\mathrm{C}$ & 1.3500586749 & 2.0487694052 & 2.8522211408 \\
\hline $\mathrm{C}$ & 2.5146832535 & 1.7135400285 & 1.9191610281 \\
\hline $\mathrm{H}$ & 2.2439364021 & 0.9235551978 & 1.2252547111 \\
\hline $\mathrm{H}$ & 3.3411294007 & 1.3526967822 & 2.5353164086 \\
\hline $\mathrm{H}$ & 2.8597887076 & 2.5941345279 & 1.3738671163 \\
\hline $\mathrm{C}$ & 0.829815886 & 0.7997776685 & 3.5751579945 \\
\hline $\mathrm{H}$ & 0.4997685334 & 0.0593874034 & 2.850364111 \\
\hline $\mathrm{H}$ & 0.0031549893 & 1.0504166412 & 4.2453020174 \\
\hline $\mathrm{H}$ & 1.6363963513 & 0.3732706175 & 4.1764856449 \\
\hline $\mathrm{C}$ & 1.8427736149 & 3.0861579888 & 3.8783973486 \\
\hline $\mathrm{H}$ & 2.7546053612 & 2.692796085 & 4.3291501017 \\
\hline $\mathrm{H}$ & 1.1370108416 & 3.2538342474 & 4.6944984305 \\
\hline $\mathrm{H}$ & 2.0900490067 & 4.0423507835 & 3.4104608139 \\
\hline $\mathrm{C}$ & -2.5131458602 & 3.1533175301 & -0.4275600833 \\
\hline $\mathrm{C}$ & -3.3308995147 & 1.8538872083 & -0.3974421825 \\
\hline $\mathrm{H}$ & -2.7307022269 & 0.9640563369 & -0.5724714401 \\
\hline $\mathrm{H}$ & -4.0971939363 & 1.899628671 & -1.1747756985 \\
\hline $\mathrm{H}$ & -3.8284106472 & 1.7345476523 & 0.5667785926 \\
\hline $\mathrm{C}$ & -1.8146597118 & 3.3841644892 & -1.7761680269 \\
\hline $\mathrm{H}$ & -1.2501921644 & 4.3190150416 & -1.7687414564 \\
\hline $\mathrm{H}$ & -2.5806662161 & 3.4600008185 & -2.5508629042 \\
\hline $\mathrm{H}$ & -1.1401538002 & 2.5809207732 & -2.0600465461 \\
\hline $\mathrm{C}$ & -3.4768055643 & 4.3315994993 & -0.2007627891 \\
\hline $\mathrm{H}$ & -4.0834336911 & 4.2090354597 & 0.6984572011 \\
\hline $\mathrm{H}$ & -4.1628417225 & 4.3623943209 & -1.0479261362 \\
\hline $\mathrm{H}$ & -2.9574826121 & 5.2919823753 & -0.1628339654 \\
\hline $\mathrm{C}$ & 0.0743028921 & 1.2501484492 & -0.1041796617 \\
\hline $\mathrm{H}$ & -0.784316155 & 1.1204291 & -0.7769501087 \\
\hline $\mathrm{C}$ & 1.2167938047 & 1.7467951697 & -1.0210110236 \\
\hline $\mathrm{C}$ & 1.8845831175 & 0.784712748 & -1.7834102347 \\
\hline $\mathrm{H}$ & 1.6131872346 & -0.2557243813 & -1.6640884569 \\
\hline $\mathrm{C}$ & 2.8922274366 & 1.1427159039 & -2.6736385458 \\
\hline $\mathrm{H}$ & 3.4002177312 & 0.3862825271 & -3.2576462387 \\
\hline $\mathrm{C}$ & 3.2423348177 & 2.4837188697 & -2.8001747864 \\
\hline
\end{tabular}




\begin{tabular}{|c|c|c|c|}
\hline $\mathrm{C}$ & 2.5998086617 & 3.4622381022 & -2.052595546 \\
\hline $\mathrm{H}$ & 2.8840214117 & 4.5020631088 & -2.1507857011 \\
\hline $\mathrm{C}$ & 1.588072316 & 3.0840800459 & -1.1695510446 \\
\hline $\mathrm{H}$ & 1.1023487172 & 3.8596806974 & -0.586880899 \\
\hline $\mathrm{C}$ & -2.3729494019 & -1.0938910393 & 1.6459839495 \\
\hline $\mathrm{H}$ & -2.1305916346 & -0.1237947094 & 2.1031527194 \\
\hline $\mathrm{H}$ & -3.2242583587 & -0.9039645092 & 0.9794802392 \\
\hline $\mathrm{C}$ & -4.6045735599 & -1.506655742 & 3.7596761366 \\
\hline $\mathrm{H}$ & -4.4593165156 & -0.4960262908 & 4.1562989099 \\
\hline $\mathrm{H}$ & -4.9798111942 & -2.1316936861 & 4.5771839559 \\
\hline $\mathrm{H}$ & -5.3908177933 & -1.4510224057 & 2.999529382 \\
\hline $\mathrm{C}$ & -1.7392260155 & -2.2958304433 & 4.455277599 \\
\hline $\mathrm{H}$ & -0.7955347939 & -2.7445803424 & 4.1342632805 \\
\hline $\mathrm{H}$ & -2.1326274054 & -2.9019799338 & 5.2779495189 \\
\hline $\mathrm{H}$ & -1.5163960148 & -1.3020573534 & 4.8576838993 \\
\hline $\mathrm{C}$ & -3.3558841321 & -3.9622067973 & 2.439667888 \\
\hline $\mathrm{H}$ & -4.0821235804 & -3.9690206147 & 1.6209129195 \\
\hline $\mathrm{H}$ & -3.7721878039 & -4.5668927832 & 3.2521213149 \\
\hline $\mathrm{H}$ & -2.4511815304 & -4.4635132272 & 2.0847940885 \\
\hline $\mathrm{C}$ & 0.4529263187 & -3.0151012963 & 1.2045682541 \\
\hline $\mathrm{H}$ & 0.6020549014 & -2.7773407457 & 2.2664666064 \\
\hline $\mathrm{H}$ & -0.152190118 & -3.933057109 & 1.1997276246 \\
\hline $\mathrm{C}$ & 2.7740877839 & -5.0568598413 & 1.325160134 \\
\hline $\mathrm{H}$ & 2.8522945746 & -4.9389028776 & 2.4109217567 \\
\hline $\mathrm{H}$ & 3.7644886845 & -5.3366829783 & 0.9499480641 \\
\hline $\mathrm{H}$ & 2.0985594683 & -5.8974635641 & 1.1359180822 \\
\hline $\mathrm{C}$ & 3.4152952107 & -2.1085983442 & 0.8473201387 \\
\hline $\mathrm{H}$ & 3.1330597512 & -1.1652052705 & 0.3743506823 \\
\hline $\mathrm{H}$ & 4.4025178892 & -2.3962083614 & 0.4708638196 \\
\hline $\mathrm{H}$ & 3.5134541878 & -1.9236087189 & 1.9221411781 \\
\hline $\mathrm{C}$ & 2.0942755962 & -3.7857974671 & -1.3620743524 \\
\hline $\mathrm{H}$ & 1.3691194901 & -4.5653862465 & -1.6143456899 \\
\hline $\mathrm{H}$ & 3.0739688142 & -4.1188721384 & -1.7203595759 \\
\hline $\mathrm{H}$ & 1.8206835656 & -2.8903809404 & -1.9270457783 \\
\hline $\mathrm{C}$ & -1.2280273266 & -1.6799959747 & -1.5390792041 \\
\hline $\mathrm{H}$ & -1.5584072073 & -0.6845894155 & -1.8728819013 \\
\hline $\mathrm{H}$ & -0.2845791175 & -1.8651732485 & -2.0684940555 \\
\hline $\mathrm{C}$ & -2.4992445296 & -2.8697146202 & -4.1071665483 \\
\hline $\mathrm{H}$ & -2.7406061379 & -1.8700763393 & -4.4835468489 \\
\hline $\mathrm{H}$ & -3.2378915055 & -3.5663534772 & -4.518137708 \\
\hline $\mathrm{H}$ & -1.5215822733 & -3.145213338 & -4.5156306239 \\
\hline $\mathrm{C}$ & -2.0940456617 & -4.6808267575 & -1.6768103561 \\
\hline $\mathrm{H}$ & -1.0948247377 & -4.9813487969 & -2.0065244609 \\
\hline $\mathrm{H}$ & -2.810352256 & -5.3849971769 & -2.1127234656 \\
\hline $\mathrm{H}$ & -2.1326316256 & -4.800131155 & -0.590535237 \\
\hline $\mathrm{C}$ & -4.2387996226 & -2.4761573196 & -1.6267940702 \\
\hline $\mathrm{H}$ & -4.3278454745 & -2.5218826592 & -0.5382394266 \\
\hline $\mathrm{H}$ & -4.9691491331 & -3.173557449 & -2.0499169998 \\
\hline $\mathrm{H}$ & -4.5294774957 & -1.468182633 & -1.9417930254 \\
\hline
\end{tabular}


2. Model III $_{\mathrm{Ar}}$

\begin{tabular}{|c|c|c|c|}
\hline $\mathrm{Ga}$ & 0.9472765579 & 1.5875573901 & -1.1763465639 \\
\hline $\mathrm{Br}$ & -6.0904118933 & -2.7712237557 & -0.8471755938 \\
\hline $\mathrm{Si}$ & -1.3664490712 & 4.0464675979 & -1.7526352244 \\
\hline $\mathrm{Si}$ & 3.7844670333 & 2.478428543 & 0.5855040045 \\
\hline $\mathrm{Si}$ & 1.5279433261 & -0.7066477805 & -3.7141296898 \\
\hline $\mathrm{O}$ & -0.1574393637 & 0.6234655328 & 0.1844299018 \\
\hline $\mathrm{N}$ & 1.7617401582 & -2.537492537 & 2.7716826123 \\
\hline $\mathrm{N}$ & 0.2626749129 & -0.9338171052 & 2.8540180692 \\
\hline $\mathrm{C}$ & 1.1095899794 & -1.6891386873 & 3.568373829 \\
\hline $\mathrm{H}$ & 1.2493863055 & -1.6189157563 & 4.6278776269 \\
\hline $\mathrm{C}$ & 0.3837874577 & -1.312153057 & 1.5064724535 \\
\hline $\mathrm{C}$ & 1.3170137391 & -2.3078184466 & 1.4844501707 \\
\hline $\mathrm{H}$ & 1.7019636477 & -2.8437033285 & 0.6357832127 \\
\hline $\mathrm{C}$ & -0.5227517163 & 0.2121912978 & 3.4923799086 \\
\hline $\mathrm{C}$ & -0.6570388781 & -0.0600570557 & 4.9988324659 \\
\hline $\mathrm{H}$ & -1.2933735404 & 0.7179659123 & 5.4218218126 \\
\hline $\mathrm{H}$ & 0.2967856675 & -0.0055290854 & 5.5299803221 \\
\hline $\mathrm{H}$ & -1.1322722445 & -1.0237205262 & 5.2014006122 \\
\hline $\mathrm{C}$ & 0.2629681126 & 1.5058415085 & 3.2435570698 \\
\hline $\mathrm{H}$ & -0.2855642491 & 2.3464420719 & 3.6754623871 \\
\hline $\mathrm{H}$ & 0.372387593 & 1.6661925873 & 2.1717508189 \\
\hline $\mathrm{H}$ & 1.24996221 & 1.4654112774 & 3.7123532618 \\
\hline $\mathrm{C}$ & -1.9172696464 & 0.2956059131 & 2.8692904033 \\
\hline $\mathrm{H}$ & -2.4460357723 & -0.6580170985 & 2.9270593293 \\
\hline $\mathrm{H}$ & -1.8564729259 & 0.6195970539 & 1.8340848899 \\
\hline $\mathrm{H}$ & -2.4942517929 & 1.0403838986 & 3.4218893003 \\
\hline $\mathrm{C}$ & 2.8000684272 & -3.5508790909 & 3.1672174647 \\
\hline $\mathrm{C}$ & 3.0562230317 & -3.4583949542 & 4.6753472707 \\
\hline $\mathrm{H}$ & 3.4411720631 & -2.4772295467 & 4.9631301357 \\
\hline $\mathrm{H}$ & 3.8109522741 & -4.1984630429 & 4.9450249513 \\
\hline $\mathrm{H}$ & 2.1582982727 & -3.6796966754 & 5.2577332101 \\
\hline $\mathrm{C}$ & 4.0894965621 & -3.2338825761 & 2.3934267947 \\
\hline $\mathrm{H}$ & 3.9470507838 & -3.3226237689 & 1.3150867149 \\
\hline $\mathrm{H}$ & 4.8700181051 & -3.9395688572 & 2.6855681472 \\
\hline $\mathrm{H}$ & 4.4355623201 & -2.2208627074 & 2.6068350752 \\
\hline $\mathrm{C}$ & 2.2616464511 & -4.9446588529 & 2.807074118 \\
\hline $\mathrm{H}$ & 1.3339403742 & -5.1576902519 & 3.3432143426 \\
\hline $\mathrm{H}$ & 2.998546747 & -5.7023853549 & 3.0811551144 \\
\hline $\mathrm{H}$ & 2.0698942187 & -5.0383369892 & 1.7365232226 \\
\hline $\mathrm{C}$ & -0.2754608916 & -0.7399671276 & 0.2479058246 \\
\hline $\mathrm{H}$ & 0.3040897233 & -1.2345838541 & -0.5528907389 \\
\hline $\mathrm{C}$ & -1.7166963886 & -1.2574718989 & 0.0440406424 \\
\hline $\mathrm{C}$ & -2.0954479776 & -2.5683860934 & 0.3446418755 \\
\hline $\mathrm{H}$ & -1.3826065127 & -3.2561243769 & 0.7880479968 \\
\hline $\mathrm{C}$ & -3.3863774509 & -3.0251150474 & 0.0860974312 \\
\hline $\mathrm{H}$ & -3.670314504 & -4.0419402141 & 0.3257945935 \\
\hline $\mathrm{C}$ & -4.3064666297 & -2.1553195404 & -0.4888689576 \\
\hline $\mathrm{C}$ & -3.95310776 & -0.8494864474 & -0.8089215089 \\
\hline
\end{tabular}




\begin{tabular}{|c|c|c|c|}
\hline $\mathrm{H}$ & -4.6751002026 & -0.1823862607 & -1.2622128764 \\
\hline $\mathrm{C}$ & -2.6584996713 & -0.4104144265 & -0.5394692857 \\
\hline $\mathrm{H}$ & -2.3611648319 & 0.6032368197 & -0.7743867361 \\
\hline $\mathrm{C}$ & -0.4311924611 & 2.5347222542 & -2.3418407928 \\
\hline $\mathrm{H}$ & 0.0938913037 & 2.8261644784 & -3.2625422259 \\
\hline $\mathrm{H}$ & -1.1591968118 & 1.7736671297 & -2.6534442175 \\
\hline $\mathrm{C}$ & -2.1262420146 & 3.8144261717 & -0.0268485098 \\
\hline $\mathrm{H}$ & -2.8160097571 & 2.9650776561 & -0.0017458384 \\
\hline $\mathrm{H}$ & -2.6884709009 & 4.7049202946 & 0.2742602454 \\
\hline $\mathrm{H}$ & -1.3543477865 & 3.6322475885 & 0.7258203416 \\
\hline $\mathrm{C}$ & -0.2408904723 & 5.5720084421 & -1.6925957717 \\
\hline $\mathrm{H}$ & 0.5579068731 & 5.4538087133 & -0.9557134091 \\
\hline $\mathrm{H}$ & -0.8094707279 & 6.4700205549 & -1.4288410415 \\
\hline $\mathrm{H}$ & 0.2293213459 & 5.7532259239 & -2.6646537206 \\
\hline $\mathrm{C}$ & -2.8009563335 & 4.4515441236 & -2.9347362257 \\
\hline $\mathrm{H}$ & -2.4347915164 & 4.6285730819 & -3.9511832796 \\
\hline $\mathrm{H}$ & -3.3492637528 & 5.3455116372 & -2.6182087356 \\
\hline $\mathrm{H}$ & -3.5173697873 & 3.6248702876 & -2.9863902061 \\
\hline $\mathrm{C}$ & 2.0789107455 & 2.8793238657 & -0.0598196182 \\
\hline $\mathrm{H}$ & 2.1925281957 & 3.7749049332 & -0.6863011903 \\
\hline $\mathrm{H}$ & 1.4462049819 & 3.1985709486 & 0.778832176 \\
\hline $\mathrm{C}$ & 5.0758591387 & 2.521044648 & -0.8028810684 \\
\hline $\mathrm{H}$ & 4.8761316476 & 1.7630620765 & -1.5652761031 \\
\hline $\mathrm{H}$ & 6.0836000011 & 2.3440193808 & -0.4125673225 \\
\hline $\mathrm{H}$ & 5.0821377919 & 3.4957017308 & -1.3012868334 \\
\hline $\mathrm{C}$ & 4.3351116326 & 3.7355229169 & 1.9046081464 \\
\hline $\mathrm{H}$ & 4.3290577237 & 4.7527922435 & 1.500374145 \\
\hline $\mathrm{H}$ & 5.347177213 & 3.5306752136 & 2.2708049101 \\
\hline $\mathrm{H}$ & 3.6607485805 & 3.7266799307 & 2.7677824284 \\
\hline $\mathrm{C}$ & 3.8967938117 & 0.7634468641 & 1.4101026048 \\
\hline $\mathrm{H}$ & 3.1881996978 & 0.6773244765 & 2.2401658691 \\
\hline $\mathrm{H}$ & 4.9034908344 & 0.5921882927 & 1.8071589954 \\
\hline $\mathrm{H}$ & 3.6738704788 & -0.029265412 & 0.6909599815 \\
\hline $\mathrm{C}$ & 2.0540852524 & 0.1390599294 & -2.1270978533 \\
\hline $\mathrm{H}$ & 3.0016803864 & 0.6469328051 & -2.3557613445 \\
\hline $\mathrm{H}$ & 2.3256903572 & -0.626727876 & -1.3862319873 \\
\hline $\mathrm{C}$ & 1.4611557343 & 0.5278392764 & -5.148995746 \\
\hline $\mathrm{H}$ & 0.7007233119 & 1.2943899917 & -4.9792710377 \\
\hline $\mathrm{H}$ & 1.2241476753 & 0.0237373787 & -6.0916556842 \\
\hline $\mathrm{H}$ & 2.4219965831 & 1.0364326488 & -5.2784974647 \\
\hline $\mathrm{C}$ & 2.7768696971 & -2.0639853077 & -4.1910486224 \\
\hline $\mathrm{H}$ & 3.7819157487 & -1.6487994184 & -4.3185061263 \\
\hline $\mathrm{H}$ & 2.5008902668 & -2.5581745367 & -5.1288990357 \\
\hline $\mathrm{H}$ & 2.8391734194 & -2.8379415059 & -3.4178466298 \\
\hline $\mathrm{C}$ & -0.1652174112 & -1.5517151196 & -3.5760349563 \\
\hline $\mathrm{H}$ & -0.173584126 & -2.3317023583 & -2.8071538469 \\
\hline $\mathrm{H}$ & -0.4372022496 & -2.0297901596 & -4.5229800629 \\
\hline $\mathrm{H}$ & -0.9554208037 & -0.8384324034 & -3.3270478038 \\
\hline
\end{tabular}


3. Model $\mathrm{II}_{\mathrm{CF} 3}$

$\begin{array}{llll}\mathrm{Ga} & 1.5378564177 & 1.9758338714 & -0.2056220116 \\ \mathrm{Si} & 1.9457843917 & 0.779283312 & 2.921862596 \\ \mathrm{Si} & 4.6066372797 & 2.7536406628 & -1.3319589057 \\ \mathrm{Si} & -0.4656087638 & 4.6406611592 & -0.9831524847 \\ \mathrm{~F} & -3.2533940514 & 0.4917065203 & -1.2842364489 \\ \mathrm{~F} & -2.3754753302 & -1.3775669018 & -0.5829001001 \\ \mathrm{~F} & -1.7077622918 & 0.514345642 & 0.2399004106 \\ \mathrm{O} & 0.0113869869 & 0.5922756561 & -1.7284525053 \\ \mathrm{C} & 1.8022208918 & 0.4516213509 & 1.0743508957 \\ \mathrm{C} & 2.4332206572 & -0.8139552456 & 3.8285649429 \\ \mathrm{H} & 1.6857304815 & -1.5975426076 & 3.669193246 \\ \mathrm{H} & 2.5211133878 & -0.650455842 & 4.9078355892 \\ \mathrm{H} & 3.3949012293 & -1.1961958526 & 3.4717417113 \\ \mathrm{C} & 0.2915870414 & 1.3735529949 & 3.6255970105 \\ \mathrm{H} & -0.0084888886 & 2.335142868 & 3.2002549096 \\ \mathrm{H} & 0.3506476663 & 1.4949655176 & 4.7121236852 \\ \mathrm{H} & -0.5069989053 & 0.6553020413 & 3.4152388697 \\ \mathrm{C} & 3.2635256052 & 2.0904314941 & 3.2966533325 \\ \mathrm{H} & 4.2483309602 & 1.7954207611 & 2.9213501408 \\ \mathrm{H} & 3.3589676308 & 2.2486924646 & 4.3756875703 \\ \mathrm{H} & 3.0136203425 & 3.0570617105 & 2.8476291203 \\ \mathrm{C} & 2.8527855585 & 2.1770099468 & -1.703268042 \\ \mathrm{C} & 5.5502789096 & 1.4142972779 & -0.3816562271 \\ \mathrm{H} & 5.0725474378 & 1.1813710726 & 0.573970504 \\ \mathrm{H} & 6.5758653109 & 1.7330822075 & -0.1699964993 \\ \mathrm{H} & 5.605936339 & 0.4854233951 & -0.958258841 \\ \mathrm{C} & 5.5381664605 & 3.0940772368 & -2.9483214646 \\ \mathrm{H} & 5.5768058971 & 2.200449313 & -3.5793294033 \\ \mathrm{H} & 6.5691539264 & 3.4094694274 & -2.756909197 \\ \mathrm{H} & 5.0515105865 & 3.8857058672 & -3.5267782612 \\ \mathrm{C} & 4.6076154532 & 4.3472559015 & -0.30819639 \\ \mathrm{H} & 4.0568513031 & 5.1457671756 & -0.8141307977 \\ \mathrm{H} & 5.628310947 & 4.7053918747 & -0.13983525 \\ \mathrm{H} & 4.148824386 & 4.1992166441 & 0.674414164 \\ \mathrm{C} & 0.2355215895 & 3.4271870944 & 0.2702924055 \\ \mathrm{C} & -1.7169380843 & 5.7856748415 & -0.1348054299 \\ \mathrm{H} & -2.5494740699 & 5.2167895447 & 0.2911581804 \\ \mathrm{H} & -2.1372653764 & 6.5128517263 & -0.8373615587 \\ \mathrm{H} & -1.2505211579 & 6.3455833545 & 0.6819515122 \\ \mathrm{C} & -1.3641836134 & 3.7493562404 & -2.3946005921 \\ \mathrm{H} & -0.6809396891 & 3.124801559 & -2.9771996932 \\ \mathrm{H} & -1.8194318546 & 4.4707676909 & -3.0805686057 \\ \mathrm{H} & 0.9018021641 & 3.108178964 & -2.0167902224 \\ \mathrm{H} & 1.4560084825 & 6.2505249051 & -0.9474917164 \\ \mathrm{H} & -1.0183214878 & 0.0051215563 & -1.9913661552\end{array}$




\begin{tabular}{|c|c|c|c|}
\hline $\mathrm{C}$ & -1.2898955471 & -0.6078599999 & -3.3066318953 \\
\hline $\mathrm{C}$ & -2.4645268415 & -1.32160422 & -3.5965229058 \\
\hline $\mathrm{H}$ & -3.2317222268 & -1.455837563 & -2.8474150967 \\
\hline $\mathrm{C}$ & -2.6485633123 & -1.8686186898 & -4.8608780945 \\
\hline $\mathrm{H}$ & -3.5550042778 & -2.4200819304 & -5.079399022 \\
\hline $\mathrm{C}$ & -1.671952655 & -1.7101270365 & -5.8419530906 \\
\hline $\mathrm{H}$ & -1.8215439968 & -2.1382236488 & -6.8266314007 \\
\hline $\mathrm{C}$ & -0.5016446139 & -1.0019078212 & -5.5614019831 \\
\hline $\mathrm{H}$ & 0.2561548127 & -0.8785105076 & -6.3258693341 \\
\hline $\mathrm{C}$ & -0.3095790378 & -0.4547429268 & -4.3024969621 \\
\hline $\mathrm{H}$ & 0.5901658792 & 0.0988323916 & -4.0662081411 \\
\hline $\mathrm{C}$ & -2.1060242358 & -0.092427455 & -0.879344113 \\
\hline $\mathrm{H}$ & 0.9416503204 & -0.2150305689 & 0.936919198 \\
\hline $\mathrm{H}$ & 2.6818476262 & -0.110702366 & 0.7392014361 \\
\hline $\mathrm{H}$ & 2.9299572479 & 1.1881919869 & -2.1737615491 \\
\hline $\mathrm{H}$ & 2.4178120877 & 2.8469694007 & -2.4538656318 \\
\hline $\mathrm{H}$ & 0.7556297386 & 4.0196856494 & 1.0384049684 \\
\hline $\mathrm{H}$ & -0.593574161 & 2.9399801048 & 0.7961423931 \\
\hline $\mathrm{H}$ & -4.7731968403 & -4.60526413 & 4.0918043663 \\
\hline $\mathrm{C}$ & -4.5795670709 & -3.5494579986 & 4.2950824145 \\
\hline $\mathrm{C}$ & -3.9286382583 & -2.878706978 & 3.0716839792 \\
\hline $\mathrm{H}$ & -5.5296767581 & -3.0629094064 & 4.5303806148 \\
\hline $\mathrm{H}$ & -3.9423551893 & -3.4834729758 & 5.1804775866 \\
\hline $\mathrm{N}$ & -2.6508402374 & -3.5796283907 & 2.7659396027 \\
\hline $\mathrm{C}$ & -4.8723097794 & -2.9775228519 & 1.8679185382 \\
\hline $\mathrm{C}$ & -3.6225658151 & -1.398922437 & 3.3663538887 \\
\hline $\mathrm{C}$ & -2.3822124079 & -4.2235229548 & 1.5921768293 \\
\hline $\mathrm{C}$ & -1.5849405425 & -3.6510652055 & 3.6607514807 \\
\hline $\mathrm{H}$ & -4.4310569368 & -2.5169260242 & 0.9844113512 \\
\hline $\mathrm{H}$ & -5.8063935152 & -2.4635378213 & 2.1123722647 \\
\hline $\mathrm{H}$ & -5.0893371663 & -4.0165915796 & 1.6207835175 \\
\hline $\mathrm{H}$ & -2.9721320889 & -1.2837425761 & 4.2368229618 \\
\hline $\mathrm{H}$ & -4.5493704692 & -0.8559232338 & 3.5687234898 \\
\hline $\mathrm{H}$ & -3.1300195988 & -0.9343185567 & 2.5094355404 \\
\hline $\mathrm{N}$ & -1.1123033898 & -4.6894778127 & 1.7821914228 \\
\hline $\mathrm{C}$ & -0.6124339671 & -4.3559883092 & 3.0397222958 \\
\hline $\mathrm{H}$ & -1.5897921588 & -3.2063864343 & 4.6410405452 \\
\hline $\mathrm{C}$ & -0.3653549032 & -5.5132520861 & 0.7922168148 \\
\hline $\mathrm{H}$ & 0.3662657822 & -4.6308356262 & 3.3933062856 \\
\hline $\mathrm{C}$ & 1.0349779986 & -4.9076062754 & 0.5877744664 \\
\hline $\mathrm{C}$ & -1.1179828982 & -5.5161257628 & -0.5434657919 \\
\hline $\mathrm{C}$ & -0.2559010773 & -6.950921303 & 1.3326064616 \\
\hline $\mathrm{H}$ & 0.9604280994 & -3.8684528998 & 0.259150585 \\
\hline $\mathrm{H}$ & 1.5756828059 & -5.4725830778 & -0.1756121888 \\
\hline $\mathrm{H}$ & 1.632718723 & -4.9335615573 & 1.501761333 \\
\hline $\mathrm{H}$ & -1.2244843167 & -4.5028760576 & -0.9333402932 \\
\hline $\mathrm{H}$ & -2.1199614965 & -5.9277392379 & -0.430896905 \\
\hline $\mathrm{H}$ & -0.5560508625 & -6.1189198056 & -1.2626513673 \\
\hline $\mathrm{H}$ & 0.2838518663 & -7.5845431024 & 0.6236906769 \\
\hline $\mathrm{H}$ & -1.2512239741 & -7.3737990193 & 1.4867527151 \\
\hline
\end{tabular}




\section{Model III $_{\mathrm{CF} 3}$}

\begin{tabular}{llll}
$\mathrm{Ga}$ & -0.0274884736 & 0.7386307566 & -1.4096940253 \\
$\mathrm{Si}$ & 3.3032722503 & 0.07252289 & -1.9602506793 \\
$\mathrm{Si}$ & -1.3482342634 & -0.4444408599 & -4.3706561901 \\
$\mathrm{Si}$ & -0.8768716902 & 4.0761211346 & -1.6452799811 \\
$\mathrm{~F}$ & -0.2852728282 & 2.5509764344 & 1.8329193517 \\
$\mathrm{~F}$ & 0.588333482 & 1.3803394388 & 3.4362889352 \\
$\mathrm{~F}$ & 1.4669536369 & 1.3318050993 & 1.4546183107 \\
$\mathrm{O}$ & -0.9619188371 & 0.2709203252 & 0.3664695375 \\
$\mathrm{~N}$ & 1.5904723837 & -2.5999443085 & 2.7452404612 \\
$\mathrm{~N}$ & -0.3427797489 & -2.4480786642 & 1.7118639044 \\
$\mathrm{C}$ & 0.6150328442 & -3.2789595735 & 2.1459027462 \\
$\mathrm{H}$ & 0.601803996 & -4.3420471995 & 2.020019907 \\
$\mathrm{C}$ & 0.0529781762 & -1.1347762608 & 2.0410542353 \\
$\mathrm{C}$ & 1.2490440689 & -1.2662015184 & 2.6900803663 \\
$\mathrm{H}$ & 1.8786479015 & -0.5052693793 & 3.1070492523 \\
$\mathrm{C}$ & 2.8537886982 & -3.1472627938 & 3.351283103 \\
$\mathrm{C}$ & 4.041035365 & -2.5672406304 & 2.5665369962 \\
$\mathrm{H}$ & 3.9858615138 & -2.8432803727 & 1.5116109294 \\
$\mathrm{H}$ & 4.9747193275 & -2.9568774602 & 2.977614322 \\
$\mathrm{H}$ & 4.0742768102 & -1.4783851463 & 2.6313464819 \\
$\mathrm{C}$ & 2.8963389848 & -2.7173284483 & 4.8259136538 \\
$\mathrm{H}$ & 2.9176571886 & -1.6311671672 & 4.9302323074 \\
$\mathrm{H}$ & 3.7997574902 & -3.1141816451 & 5.2934046274 \\
$\mathrm{H}$ & 2.0301741486 & -3.0993062408 & 5.370954375 \\
$\mathrm{C}$ & 2.8479403526 & -4.6763093122 & 3.2489362933 \\
$\mathrm{H}$ & 2.0072029441 & -5.1188947138 & 3.7892916895 \\
$\mathrm{H}$ & 3.7651417852 & -5.0571873221 & 3.6999621818 \\
$\mathrm{H}$ & 2.8295847061 & -5.016486315 & 2.2108410212 \\
$\mathrm{C}$ & -1.4971509436 & -3.0374423684 & 0.8764956745 \\
$\mathrm{C}$ & -0.9826140963 & -3.1688970011 & -0.5617384414 \\
$\mathrm{H}$ & -1.7719057874 & -3.589806585 & -1.188577398 \\
$\mathrm{H}$ & -0.1144594708 & -3.8314285243 & -0.6177779263 \\
$\mathrm{H}$ & -0.7057686431 & -2.1953925368 & -0.9602121055 \\
$\mathrm{C}$ & -1.8522549362 & -4.4185353725 & 1.460511996 \\
$\mathrm{H}$ & -2.7484827946 & -4.7751997736 & 0.9513679405 \\
$\mathrm{H}$ & -2.0771347692 & -4.3572806402 & 2.5280894956 \\
$\mathrm{H}$ & -1.0813211447 & -5.175256405 & 1.2964541863 \\
$\mathrm{C}$ & -2.7450074702 & -2.15956672 & 0.9289493682 \\
$\mathrm{H}$ & -2.5511675401 & -1.1828072681 & 0.4932187322 \\
$\mathrm{H}$ & -3.1170127664 & -2.0437372448 & 1.9474993707 \\
& -3.5188079624 & -2.6568924592 & 0.339353472 \\
$\mathrm{C}$ & 1.6215022124 & -0.491028473 & -1.3569590851 \\
\hline & 4.5471661678 & -1.371990339 & -1.8882202876 \\
\hline & 5.5374493082 & -1.7396156546 & -0.8630226775 \\
& & & -2.248708008
\end{tabular}




\begin{tabular}{|c|c|c|c|}
\hline $\mathrm{H}$ & 4.212055175 & -2.2143356246 & -2.5024796757 \\
\hline $\mathrm{C}$ & 4.010526412 & 1.4609988045 & -0.8810038667 \\
\hline $\mathrm{H}$ & 3.3913627375 & 2.3599054349 & -0.9316724785 \\
\hline $\mathrm{H}$ & 5.0224400558 & 1.7293180187 & -1.2022512525 \\
\hline $\mathrm{H}$ & 4.064090721 & 1.1610435878 & 0.1704596465 \\
\hline $\mathrm{C}$ & 3.2676051112 & 0.6686644145 & -3.7587656019 \\
\hline $\mathrm{H}$ & 2.8806106731 & -0.1042822071 & -4.4300252433 \\
\hline $\mathrm{H}$ & 4.2729565126 & 0.9315010838 & -4.1039693133 \\
\hline $\mathrm{H}$ & 2.6370064507 & 1.5543677856 & -3.8764258117 \\
\hline $\mathrm{C}$ & -1.5681418628 & 0.1424288687 & -2.6048665551 \\
\hline $\mathrm{C}$ & -0.3377384497 & -2.0520393549 & -4.457221627 \\
\hline $\mathrm{H}$ & 0.6814305523 & -1.9088540813 & -4.08844399 \\
\hline $\mathrm{H}$ & -0.2701884828 & -2.41250657 & -5.4890380779 \\
\hline $\mathrm{H}$ & -0.7990458437 & -2.847001803 & -3.861853351 \\
\hline $\mathrm{C}$ & -3.0388028163 & -0.8197103157 & -5.158750161 \\
\hline $\mathrm{H}$ & -3.5767976732 & -1.5896221778 & -4.5956150766 \\
\hline $\mathrm{H}$ & -2.9360625959 & -1.1731925519 & -6.1904801701 \\
\hline $\mathrm{H}$ & -3.6711751416 & 0.0738107786 & -5.176280977 \\
\hline $\mathrm{C}$ & -0.493779875 & 0.8418163317 & -5.4672070938 \\
\hline $\mathrm{H}$ & -1.0727665902 & 1.7682291365 & -5.5185268867 \\
\hline $\mathrm{H}$ & -0.379898255 & 0.4663149 & -6.4896575255 \\
\hline $\mathrm{H}$ & 0.5020855991 & 1.096735628 & -5.0955347894 \\
\hline $\mathrm{C}$ & 0.4019813374 & 2.7234612789 & -1.4207118239 \\
\hline $\mathrm{C}$ & -0.144636934 & 5.7545106468 & -1.1329110832 \\
\hline $\mathrm{H}$ & 0.1453803968 & 5.7497705205 & -0.0770831533 \\
\hline $\mathrm{H}$ & -0.8604252976 & 6.5709262166 & -1.2775257381 \\
\hline $\mathrm{H}$ & 0.7507895697 & 5.9890850597 & -1.7175994696 \\
\hline $\mathrm{C}$ & -2.4492215981 & 3.8177668803 & -0.6173590709 \\
\hline $\mathrm{H}$ & -2.9693882065 & 2.9022922477 & -0.9147681491 \\
\hline $\mathrm{H}$ & -3.1425182439 & 4.6544424973 & -0.7548959857 \\
\hline $\mathrm{H}$ & -2.2215867563 & 3.7427147305 & 0.4489171587 \\
\hline $\mathrm{C}$ & -1.4078419247 & 4.2218901185 & -3.4612614543 \\
\hline $\mathrm{H}$ & -0.5483546772 & 4.3874670071 & -4.118515744 \\
\hline $\mathrm{H}$ & -2.0969935329 & 5.0616988058 & -3.5990513492 \\
\hline $\mathrm{H}$ & -1.9159016539 & 3.316865703 & -3.8053179351 \\
\hline $\mathrm{C}$ & -0.6747339633 & 0.1903581535 & 1.688741104 \\
\hline $\mathrm{C}$ & -1.9351635009 & 0.3710503077 & 2.5882985592 \\
\hline $\mathrm{C}$ & -1.9941888174 & -0.0819366732 & 3.9083447628 \\
\hline $\mathrm{H}$ & -1.1465097418 & -0.5940243921 & 4.3491487966 \\
\hline $\mathrm{C}$ & -3.1393823959 & 0.1205182704 & 4.6770655345 \\
\hline $\mathrm{H}$ & -3.1727437023 & -0.2405725208 & 5.6993004406 \\
\hline $\mathrm{C}$ & -4.2343491763 & 0.7898611668 & 4.1360466731 \\
\hline $\mathrm{H}$ & -5.1253825659 & 0.9497232049 & 4.7329434052 \\
\hline $\mathrm{C}$ & -4.1740663091 & 1.2563821099 & 2.8241013512 \\
\hline $\mathrm{H}$ & -5.0182901655 & 1.7850319457 & 2.3955558226 \\
\hline $\mathrm{C}$ & -3.0322382438 & 1.0475998374 & 2.0553721854 \\
\hline $\mathrm{H}$ & -2.9773983593 & 1.3915580662 & 1.0318215739 \\
\hline $\mathrm{C}$ & 0.2806213931 & 1.3728431464 & 2.1042708025 \\
\hline $\mathrm{H}$ & 1.7459669085 & -0.7723499179 & -0.3032443764 \\
\hline $\mathrm{H}$ & 1.3658751158 & -1.4163878877 & -1.8883695379 \\
\hline
\end{tabular}




$\begin{array}{llll}\mathrm{H} & -2.0931909233 & -0.6536380375 & -2.0611536699 \\ \mathrm{H} & -2.2712837888 & 0.9856716143 & -2.6242177955 \\ \mathrm{H} & 1.1304555691 & 2.8477406402 & -2.2353637042 \\ \mathrm{H} & 0.9618708732 & 2.9330547416 & -0.5031688631\end{array}$

\section{Model $\mathrm{II}_{\mathrm{H}}$}

$\begin{array}{llll}\mathrm{Ga} & 1.099209068 & -0.1800694082 & -0.4538073148 \\ \mathrm{Si} & 2.870210793 & -2.8194938931 & 0.8269318049 \\ \mathrm{Si} & 1.8139805471 & 2.8830814825 & 0.8477612396 \\ \mathrm{Si} & 1.989336639 & -0.2483027978 & -3.760433853 \\ \mathrm{O} & -0.6857620239 & 0.258662169 & 0.3595086873 \\ \mathrm{~N} & -3.8711124008 & 0.8682979423 & 0.376331475 \\ \mathrm{~N} & -3.3153758517 & -0.3619363448 & 2.1037305646 \\ \mathrm{C} & -2.9797284356 & -0.0544502255 & 0.823179362 \\ \mathrm{C} & -4.7700738713 & 1.1382995312 & 1.3911026222 \\ \mathrm{H} & -5.5754035695 & 1.8384278183 & 1.289112451 \\ \mathrm{C} & -4.4270744645 & 0.3801121894 & 2.4558073471 \\ \mathrm{H} & -4.8884222444 & 0.3210454841 & 3.4215648314 \\ \mathrm{C} & -2.6359483079 & -1.3539329524 & 3.0431399787 \\ \mathrm{C} & -3.3345683502 & -1.2944799714 & 4.4112515786 \\ \mathrm{H} & -3.2781662246 & -0.3001746575 & 4.8591966916 \\ \mathrm{H} & -2.8113685419 & -1.9805930863 & 5.0784958212 \\ \mathrm{H} & -4.3782923524 & -1.6153136777 & 4.3654815058 \\ \mathrm{C} & -2.8090474124 & -2.7763151089 & 2.4820533236 \\ \mathrm{H} & -3.8652814122 & -2.9995504416 & 2.3106683842 \\ \mathrm{H} & -2.4252389016 & -3.4925477611 & 3.2116102623 \\ \mathrm{H} & -2.2646251154 & -2.9332720358 & 1.5528115629 \\ \mathrm{C} & -1.1647955424 & -0.9570152688 & 3.2282663137 \\ \mathrm{H} & -0.623804039 & -0.8657872097 & 2.2889061191 \\ \mathrm{H} & -0.6751481588 & -1.7051443847 & 3.8558671797 \\ \mathrm{H} & -1.0968165653 & 0.0102484323 & 3.7316794272 \\ \mathrm{C} & -3.9373800552 & 1.5184097098 & -1.0007333195 \\ \mathrm{C} & -5.0575555595 & 2.5710823478 & -0.9956502574 \\ \mathrm{H} & -6.0473364235 & 2.1330472668 & -0.8449371031 \\ \mathrm{H} & -5.0607245892 & 3.054750001 & -1.9732685788 \\ \mathrm{H} & -4.8858596068 & 3.3486223362 & -0.2483497159 \\ \mathrm{C} & -2.6074599657 & 2.2256105599 & -1.2963240127 \\ \mathrm{H} & -2.4545642368 & 3.0474245896 & -0.5931214302 \\ \mathrm{H} & -2.6478401671 & 2.6419100829 & -2.3055284014 \\ \mathrm{H} & -1.7436311982 & 1.5698474904 & -1.2188216718 \\ \mathrm{C} & -4.2936728558 & 0.4456424068 & -2.0449583312 \\ \mathrm{H} & -3.5039466735 & -0.291336007 & -2.1790585877 \\ \mathrm{H} & -4.4567738986 & 0.9304164439 & -3.0098474797 \\ \mathrm{H} & -5.2135065501 & -0.0749388867 & -1.76639277 \\ \mathrm{C} & 1.316949418 & -2.1704876285 & 0.0052134956 \\ \mathrm{H} & 2.9369475247 & -2.2932436855 & 2.6508664973 \\ & 2.9448184322 & -1.2051348668 & 2.7582033693 \\ & 3.8358871399 & -2.6822044844 & 3.1401842452\end{array}$




$\begin{array}{llll}\mathrm{H} & 2.0714979443 & -2.6751282329 & 3.20316125 \\ \mathrm{C} & 2.9055917154 & -4.7218709973 & 0.7990979301 \\ \mathrm{H} & 2.0269050597 & -5.1414215018 & 1.3004350914 \\ \mathrm{H} & 3.7948692519 & -5.1193256099 & 1.3002333 \\ \mathrm{H} & 2.9075312502 & -5.1014670511 & -0.2279307924 \\ \mathrm{C} & 4.4512543637 & -2.2156574983 & -0.0258249717 \\ \mathrm{H} & 4.4715937555 & -2.5019073654 & -1.0820894829 \\ \mathrm{H} & 5.3405858102 & -2.6453286512 & 0.4470484881 \\ \mathrm{H} & 4.5393116603 & -1.126955955 & 0.0250125707 \\ \mathrm{C} & 2.3537318808 & 1.13771196 & 0.4442461064 \\ \mathrm{C} & 3.2772492626 & 3.9129947703 & 1.4947448447 \\ \mathrm{H} & 3.7085786422 & 3.464723239 & 2.3958222718 \\ \mathrm{H} & 2.9743836761 & 4.9357141438 & 1.7454479837 \\ \mathrm{H} & 4.0761208579 & 3.977547953 & 0.7487335228 \\ \mathrm{C} & 1.1437529186 & 3.7896797879 & -0.6796015803 \\ \mathrm{H} & 1.894763447 & 3.8305367355 & -1.4750231077 \\ \mathrm{H} & 0.865336717 & 4.820666527 & -0.4360624294 \\ \mathrm{H} & 0.2618676751 & 3.2886274849 & -1.0866921754 \\ \mathrm{C} & 0.4747162456 & 2.8926574164 & 2.1913156499 \\ \mathrm{H} & -0.3692787908 & 2.274405299 & 1.8755795832 \\ \mathrm{H} & 0.1193300135 & 3.9077225228 & 2.3991525806 \\ \mathrm{H} & 0.8624077866 & 2.479864345 & 3.1290037482 \\ \mathrm{C} & 0.7253181094 & 0.1468434248 & -2.4376703831 \\ \mathrm{C} & 2.2827421292 & -2.1178452575 & -3.8988941736 \\ \mathrm{H} & 2.6817432165 & -2.5339439892 & -2.9699733331 \\ \mathrm{H} & 2.9944061612 & -2.3467077846 & -4.6989821255 \\ \mathrm{H} & 1.3518755188 & -2.6487708318 & -4.1244522028 \\ \mathrm{C} & 3.6503108794 & 0.601087831 & -3.4240313683 \\ \mathrm{H} & 3.525948263 & 1.6836489615 & -3.3191050496 \\ \mathrm{H} & 4.3562226896 & 0.4272758489 & -4.2428925694 \\ \mathrm{H} & 4.1125769035 & 0.2325921876 & -2.50393037 \\ \mathrm{C} & 1.388251018 & 0.3469246201 & -5.4655299401 \\ \mathrm{H} & 0.4365451343 & -0.1233044696 & -5.7349507584 \\ \mathrm{H} & 2.1095065875 & 0.1111484862 & -6.2555666882 \\ \mathrm{H} & 1.2324421786 & 1.4308112652 & -5.472245251 \\ \mathrm{C} & -1.7567448293 & -0.5537815531 & 0.0723401784 \\ \mathrm{H} & 0.4799767639 & -2.457203927 & 0.6585692732 \\ \mathrm{H} & 1.1621223179 & -2.7265767334 & -0.9282935518 \\ \mathrm{H} & 3.2354536514 & 1.2060133139 & -0.2076990862 \\ & 2.7121185463 & 0.6822125591 & 1.3763538723 \\ \mathrm{H} & -0.188672628 & -0.4094356819 & -2.695200376 \\ & -2.4700812934 & 1.2108938307 & -2.5314565769 \\ \mathrm{H} & -1.6004474862 & -0.5848879564 & -0.9968141046 \\ & & & 0.3518033406\end{array}$

\section{Model $\mathrm{III}_{\mathrm{H}}$}

$\begin{array}{llll}\mathrm{Ga} & 0.0182339213 & 1.4178525069 & 1.0592270698 \\ \mathrm{Si} & -0.0464987269 & 1.0864828149 & 4.4634237184\end{array}$




\begin{tabular}{|c|c|c|c|}
\hline $\mathrm{Si}$ & 3.0575394482 & 1.4766823783 & -0.5477659616 \\
\hline $\mathrm{Si}$ & -2.5490292883 & 3.2358567408 & -0.3734696769 \\
\hline $\mathrm{O}$ & -0.4238702794 & -0.4771886346 & 0.6996203607 \\
\hline $\mathrm{N}$ & -0.3407101073 & -2.8113176053 & -3.1286310972 \\
\hline $\mathrm{N}$ & -0.2237449107 & -3.0760483896 & -0.9545115793 \\
\hline $\mathrm{C}$ & 0.1030571202 & -3.5977106878 & -2.1450368605 \\
\hline $\mathrm{H}$ & 0.6430480506 & -4.5114397894 & -2.2893628745 \\
\hline $\mathrm{C}$ & -0.9078269019 & -1.8750972319 & -1.1819765698 \\
\hline $\mathrm{C}$ & -0.9729563873 & -1.7330436076 & -2.5368451155 \\
\hline $\mathrm{H}$ & -1.4051507829 & -0.9311066455 & -3.1073775303 \\
\hline $\mathrm{C}$ & 0.2265217922 & -3.6911095599 & 0.3661376425 \\
\hline $\mathrm{C}$ & 0.4962217848 & -5.1877779954 & 0.1439397508 \\
\hline $\mathrm{H}$ & 0.7367609835 & -5.6328360489 & 1.1101697266 \\
\hline $\mathrm{H}$ & 1.3544057056 & -5.3731380429 & -0.50733578 \\
\hline $\mathrm{H}$ & -0.3793975544 & -5.708320441 & -0.2538658766 \\
\hline $\mathrm{C}$ & 1.5081403172 & -2.9682668306 & 0.8043329595 \\
\hline $\mathrm{H}$ & 1.8424074703 & -3.3785603734 & 1.7603937693 \\
\hline $\mathrm{H}$ & 1.302430861 & -1.9043432252 & 0.9237670072 \\
\hline $\mathrm{H}$ & 2.3078897433 & -3.1120094051 & 0.072228856 \\
\hline $\mathrm{C}$ & -0.8817653374 & -3.5258574501 & 1.4128265802 \\
\hline $\mathrm{H}$ & -1.8341081736 & -3.9248549404 & 1.053859734 \\
\hline $\mathrm{H}$ & -0.9897773714 & -2.4812343476 & 1.6980688698 \\
\hline $\mathrm{H}$ & -0.5949986276 & -4.0874821487 & 2.3041412312 \\
\hline $\mathrm{C}$ & -0.1752267371 & -3.0194249318 & -4.6077017276 \\
\hline $\mathrm{C}$ & 0.6152645144 & -4.3084093123 & -4.8585580246 \\
\hline $\mathrm{H}$ & 1.6185408802 & -4.2614781578 & -4.4281953385 \\
\hline $\mathrm{H}$ & 0.727070048 & -4.4437361454 & -5.9352519527 \\
\hline $\mathrm{H}$ & 0.0973740443 & -5.1891219014 & -4.4702129 \\
\hline $\mathrm{C}$ & 0.5915937328 & -1.8125458099 & -5.1713523239 \\
\hline $\mathrm{H}$ & 0.0370545804 & -0.8826863642 & -5.0346192447 \\
\hline $\mathrm{H}$ & 0.7522270653 & -1.9518510029 & -6.2424438658 \\
\hline $\mathrm{H}$ & 1.5622209086 & -1.7027133802 & -4.683863716 \\
\hline $\mathrm{C}$ & -1.5760726141 & -3.130088621 & -5.2305804131 \\
\hline $\mathrm{H}$ & -2.1274457539 & -3.9726509703 & -4.8070484144 \\
\hline $\mathrm{H}$ & -1.4857666154 & -3.2842822806 & -6.3078066709 \\
\hline $\mathrm{H}$ & -2.1600748526 & -2.2218535337 & -5.0712343785 \\
\hline $\mathrm{C}$ & -1.4196415845 & -0.8863409246 & -0.1534340987 \\
\hline $\mathrm{H}$ & -1.8577591439 & -0.0709990433 & -0.7505575918 \\
\hline $\mathrm{C}$ & -0.7668149359 & 1.8170920503 & 2.9009142698 \\
\hline $\mathrm{H}$ & -0.7474893584 & 2.9104009861 & 3.0143825683 \\
\hline $\mathrm{H}$ & -1.8302786956 & 1.5453423297 & 2.8536505012 \\
\hline $\mathrm{C}$ & 0.1221028295 & -0.8025312207 & 4.3613093831 \\
\hline $\mathrm{H}$ & -0.8473577813 & -1.2722996779 & 4.166832222 \\
\hline $\mathrm{H}$ & 0.5129264769 & -1.2161151328 & 5.2972040248 \\
\hline $\mathrm{H}$ & 0.7965540285 & -1.0921722684 & 3.5511168482 \\
\hline $\mathrm{C}$ & 1.6657884671 & 1.8093407424 & 4.8444647333 \\
\hline $\mathrm{H}$ & 2.3873864121 & 1.566534452 & 4.060092957 \\
\hline $\mathrm{H}$ & 2.0555543583 & 1.4237288817 & 5.7925660983 \\
\hline $\mathrm{H}$ & 1.6224432209 & 2.9004855889 & 4.9255144689 \\
\hline $\mathrm{C}$ & -1.1553563077 & 1.4582535446 & 5.9655761966 \\
\hline
\end{tabular}




$\begin{array}{llll}\mathrm{H} & -1.2729668931 & 2.5372213423 & 6.1099167997 \\ \mathrm{H} & -0.7404559757 & 1.042804247 & 6.8906156308 \\ \mathrm{H} & -2.1570338582 & 1.036207179 & 5.8321581183 \\ \mathrm{C} & 2.0639774695 & 1.4010328073 & 1.0295594479 \\ \mathrm{H} & 2.3859021281 & 2.2449846364 & 1.6555397267 \\ \mathrm{H} & 2.3594535885 & 0.4995857205 & 1.5840295386 \\ \mathrm{C} & 2.9415757417 & 3.1800157818 & -1.3742110335 \\ \mathrm{H} & 1.9197822679 & 3.4021603209 & -1.6932414845 \\ \mathrm{H} & 3.5890978481 & 3.238231815 & -2.2556321789 \\ \mathrm{H} & 3.2515220244 & 3.9723476721 & -0.6851819406 \\ \mathrm{C} & 4.9026571244 & 1.1368886331 & -0.2238054438 \\ \mathrm{H} & 5.3162838553 & 1.8681531234 & 0.478051758 \\ \mathrm{H} & 5.4968937449 & 1.1863529878 & -1.1430845282 \\ \mathrm{H} & 5.0513310252 & 0.1450200923 & 0.2163368468 \\ \mathrm{C} & 2.499063415 & 0.1805886695 & -1.8280875201 \\ \mathrm{H} & 2.576458388 & -0.8311041109 & -1.4172686562 \\ \mathrm{H} & 3.1219599952 & 0.2294321427 & -2.7282069437 \\ \mathrm{H} & 1.4584891568 & 0.3444926301 & -2.1208529663 \\ \mathrm{C} & -0.8346400587 & 2.4921854967 & -0.4719261956 \\ \mathrm{H} & -0.1449371869 & 3.3339982233 & -0.6246747843 \\ \mathrm{H} & -0.7502068466 & 1.8921948064 & -1.3896177156 \\ \mathrm{C} & -2.6050222689 & 4.6399338724 & 0.8974815279 \\ \mathrm{H} & -2.409994972 & 4.2690720312 & 1.9070173914 \\ \mathrm{H} & -3.5841105519 & 5.1302561931 & 0.9033142745 \\ \mathrm{H} & -1.8536211126 & 5.4043072683 & 0.6743106434 \\ \mathrm{C} & -3.0708973131 & 3.9679978914 & -2.0529678279 \\ \mathrm{H} & -2.3674011925 & 4.740207083 & -2.380533667 \\ \mathrm{H} & -4.0663293301 & 4.4229728188 & -2.0068500121 \\ \mathrm{H} & -3.0956713821 & 3.1976261002 & -2.8316865796 \\ \mathrm{C} & -3.8848758833 & 1.9675798118 & 0.097785954 \\ \mathrm{H} & -3.9689148096 & 1.1668697106 & -0.6447697911 \\ \mathrm{H} & -4.8665402657 & 2.4478562031 & 0.1690955647 \\ \mathrm{H} & -3.6715609185 & 1.503080711 & 1.0647746758 \\ \mathrm{H} & -2.2745909881 & -1.3504323758 & 0.3729193518\end{array}$

